cots

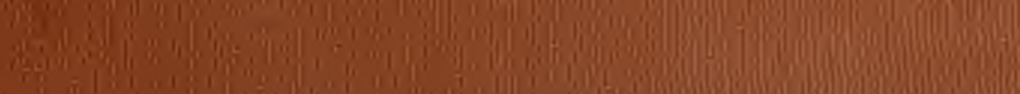
-

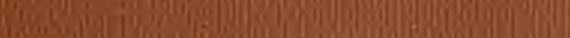
isore, a 5.0.

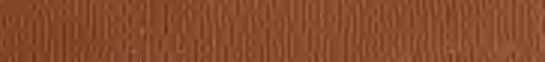
a. "Th a

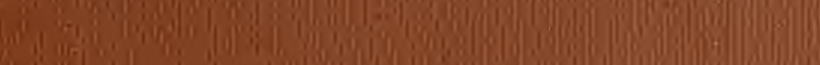

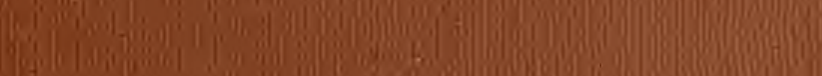
: 25: (1)

on las (10) in: cons.

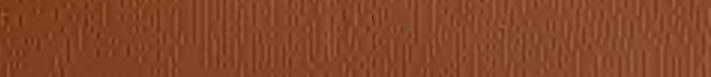

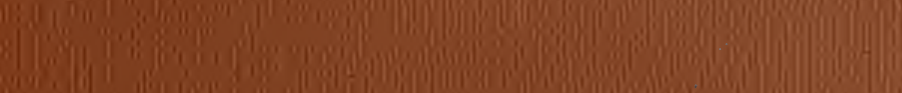
G9. Q a 1) 
(1) 


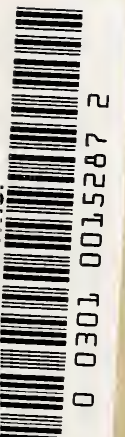




\section{FAUNE DE FRANCE 1}

ÉCHINODERMES 


\section{AVIS AU PUBLIC}

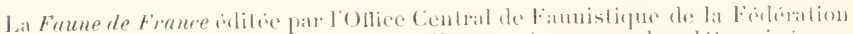

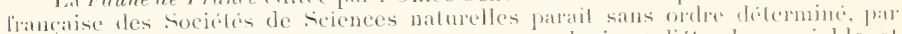

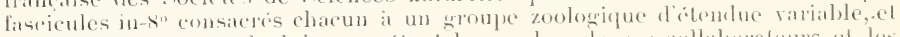

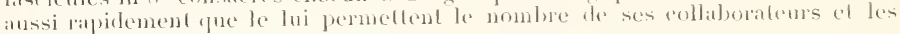
fonds is sa disposition.

Elle a pour bul de fournir aux naluralistes, sous une forme aussi portative ed anssi peu oncreuse que possible, le moven distentifier sirenent une espece

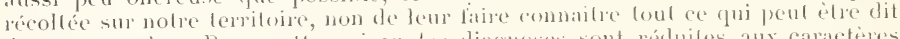
de celle espece. Ponr celle raison fes diagnoses sont réduiles aux caracteres

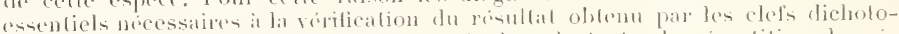

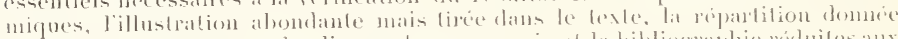

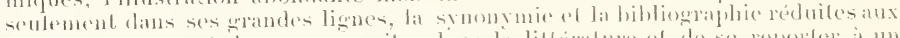

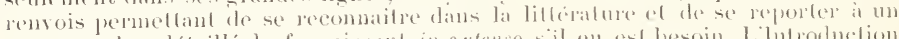

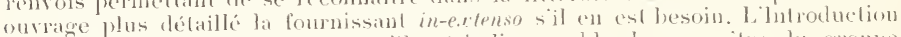
de charque volume renferme ce quil est indiopensible de comaitae du groupe au point de vue géneral el pour la détermination de ses esprecen.

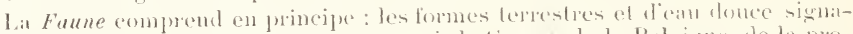

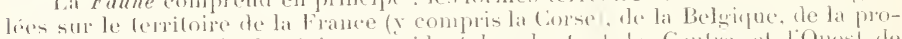

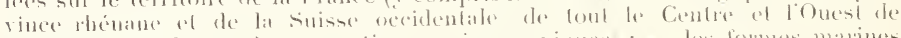

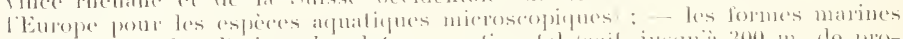

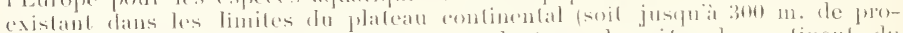

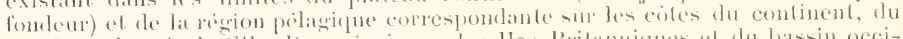
sund an difloit de Gibrallar, ainsi que des lles britanniques el du bassin occidental de la Méditerranse.

Les volumes, edités mratee à des subventions accordées dans l’intérêt de ba

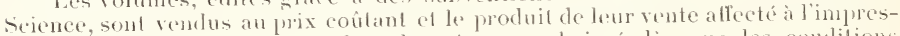
sion de nomvean fatscicules donl le prix sera abaissé dis que les conditions actuclles simélioreront.

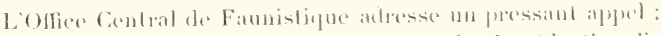

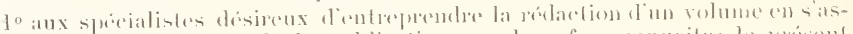

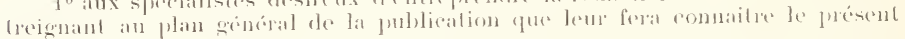
Pascicule:

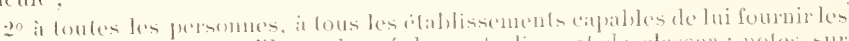

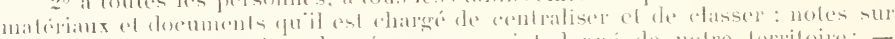

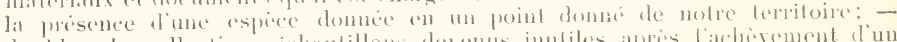
doubles de collection, achantillons derenus indiles atpris l'atherement diun

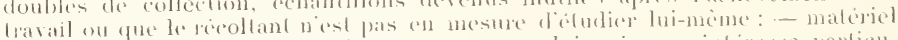

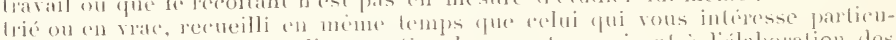

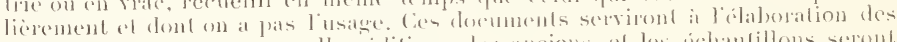

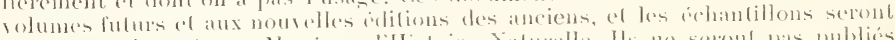

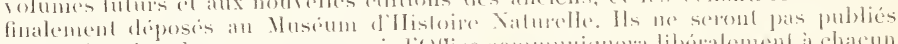

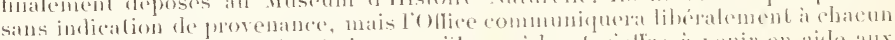
tous les renseignements litunislipues quil posside ef solfre il venir en aide aux trarailleurs isolés pour la détermination des animan qui les intéressent

Le Directeur de follice de liannistique,

P. IIE BEILCHIIIY,

Laboratoire de Zoologie, Faculte des Sciences, Disos, 


\section{OFFICE CENTRAL DE FAUNISTIQUE}

FAUNE

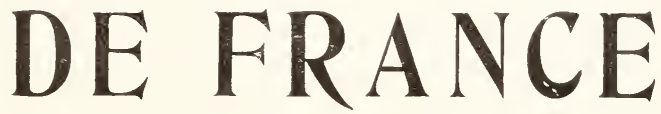

1

\section{ÉCHINODERMES}

I'A R

R. KOEHLER

PIOOFESETR A LA F.ACI LTÉ DES SEIEXEES DE I.YON

Avec 153 figures

\section{I'NRIS}

PALL LECIIEVILIEL, 12, RLE DE TOTKXWX $1 \mathrm{I}^{\circ}$ 



\section{ERRATA ET MODHICITIONS}

Hitges

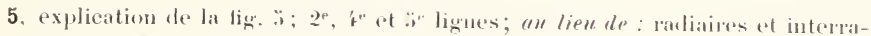
diaires lire: radiales et interradiales

7, explication de la fig. 10, fe ligne: an lien de : ambuaclraire live: ambulaeraire

13, paragraphe 2, 5e ligne; an lien de: une pl. c.-dors. lire: une pl. improprement appelée c.-tlor's.

13, 2e ligne de la note; au lien de : qui part de la c.-dorsale lire: qui part du sommet du calice et dont la plaque proximale formera la pl dite c.-dor's. ehez les Antedonidit.

19, dernière ligne; an lien de: (p.) 38 lire: (p. 38)

21. 8e ligne: au lien de: esprèce lire: espèces

21, $12^{e}$ ligne, ajonter : ....... 2't

21, au milieu de la page; an lien de: O. Forcipulées lire: 0 . Forcipulosées

24, he ligne; an lieu de: pedicellaires droits lire: pédicellaires général. croisés, parfois droits

26, 2 e ligne : supprimer : (fig. $\$ 3$ )

29, 4e ligne à partir du bas; au lieu de: squelet.: lime: squelet

35, diagnose des Solasterida, $1^{\mathrm{r}}$ ligne; au lieu de : Le suel. lre: l.e squel.

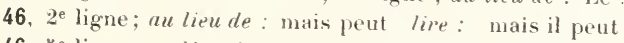

46, 5. ligne; an lieu de: remplacée lire: remplacé

47, dernière ligne; au lieu de: marg, lire: marg.

70, avant-dernière ligne; au lieu de: sur la ventr. lire: sur la face ventr.

76, explication de la fig. 46, f ligne: aprés : bras ajouter : grandeur naturelle

76, $29^{\circ}$ ligne; an lieu de: littorale lire: littorales

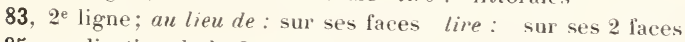

85, explication de la fig. 56 ; au lieu de $: \times 5$ lire : $\times 3$

86, 3e ligne de la description; an lieu de : le lire: ils

87, 2e ligne de la diagnose des Ophiodermatidar: an leen de : ainsi que portés lire: ansi que les orales pt portés

88, tre ligne; an lieu de: les picces huceales lure: los pl. orales et andorales

88, to ligne à partir du bas; an lien de: claire lire: claires

91, 2e ligne à partir du bas; an lien de: certaine lire: certaines

93, avant-dernière ligne de la diagnose des Ophiocomide' : an lien de : Ophiopsilidie lire: Ophiopsilina

93, Ire ligne après Ophiocomina; an lien de : 1921 / lire: 1921

94, explication de la fig. 62: an lien de: face ventrale, B lire: B. face verutrale 
94. dernione ligne du 2e parangaphe; an lien de: Sicile, lire: Sicile.

100. 2. ligne: un lieu de: lee glandes males ont une conleur orangée, les femelles sont plus pitles live: les glandes lemelles ont une couleur orangée, les miles sont plus piales

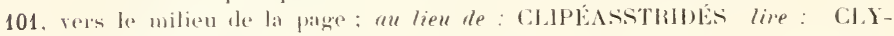
P'EASTIBHES

101, vers le milieu de la page: an lieu de: SPATAVGIDÉs lire: SPATANGIDES.

101. 6." ligne is partir du has: an lieu de: post. 3 lire: post. 5

105. 6" ligne: supprimer les mots: ì la base

106. I:"e Jigne it parlir du has: an lipu de: un peu plus courts lire: un peu plus long's

112. explication de la lig. 74 ; au lien de: Centrostehanus $p$. longispinus lire: Centrostephanus longispinus

114. 28 ligne: au lieu de : caracté-ristique live: caractéristique

116. $9^{r}$ ligne à partir du bas: an lieu de : conique vu par en haut, lire: conique: vu par en haut,

118. $6^{\circ}$ ligne; au lieu de: verts à la hase puis rouges lire: rouges à la base puis verts

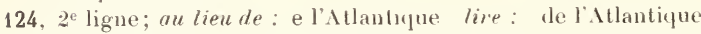

131. 2e paragraphe, 2r ligne: an lieu de : il l'extrém., étroits lire: i l'extrém., moins étroits

137. lig. 96; celte figure doil être relournese de bas en hant.

140. 3r ligne; an lien de: dn cylindre. live: du eylindre, et l'anus à l'antre extrémité.

145, je ligne ì partir du bas; au lieu de : égaliêr. live: régulièr.

147. an milieu de la page: le $\mathrm{X}^{\circ} 22$ doit être interchangé avec le trait placé an dessus.

155, explication de la lig. 106, 2" ligne' un lieu de : plaques lire: plaque

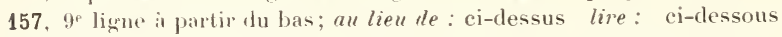

159, dernière ligne; au lien de : cules crépus lire: cule crépu

166. 9" lique i partir du bas; au lien de: défaut dans les tentac. lire: défaut dans les tég. ainsi que dans les tentac.

168, Jre ligne; au lieu de: is tentac. lire: I8 à 20 tentac.

183. 10 ligne: apres : assez grands, ajouter: dont les bords du disque sont lisses

192. fig. 147; relle tigure doit itre retoumée de bas en haut.

197. Ir" ligne; an lieu te: 150 a lire: $149 a$

199. ' $t$ "ligne; au lieu de: dans la région des cirres lire: dans la région terminale des cirres 


\section{INTRODUCTION}

Je ne puis dans cet ourrage essentiellement deseruptif m'étendre sur les caractères généraux des Échinolerme- : le petit nombre de pages dont je dispose me lanterdit formellement. Il existe l'ailleurseles publications ou le lecten tromera des renseinnements détailles sur et embranchenent : je

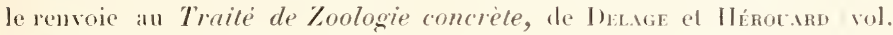
III et an Traité d'Anatomie comparée de L.Axg wol. II); je me contenterai de ruppeler ici les points quil est indispensable de commaitre pour lis détemination des Échinodermes. C'est aussi pour ne pas surcharger le texte que jai du etre tres sobre d'indications bibliographiques : je me suis borné à mentionner les mémoires dans lesquels le lectem trouvera me description an des dessins de l'espèce etudiee. Eu ce qui concerne ces indications elles-mèmes, on les trouvera principalement dans les Mémoires

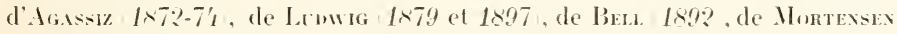

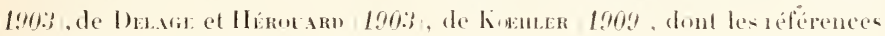
se trouvent a la tin de cet anvage.

\section{to MORPHOLOGIE}

Les Echinodermes constituent l'un des groupes les mienx caractérisés du Règne animal, et ils se reconnaisent a premiere vue. Indépendamment d'autres particularités de leur organisation, ils présentent avant tout trois caractères essentiels: ils offrent une srmétrie pentaradiée, ils pos-èdent un squelette externe formé de nombreuses plaques calcaires, et entin ils présentent un appareil très particulier qui nexiste dans aucun autre groupe du règne animal, lappareil aquifère. Examinon- rapidement ces prineipaux caractères.

On sait que chez les Coelentérés, les "antimères " sont disposés autour de la bonche au nombre de 4 ou de 6 ou d'un multiple de ces chiffres : chez les Échinodermes fig. 1), ces antimères sont au nombre de 5 : les t-téries 
ef les Ophimes ont jbras ordinarement simples c , les Crinoj̈les ont 5 bras ramities (d) : les Échinides b el les Jtololhuries a) sont comparables a une sphere ou i un evlindreavee s bandescorrespondant a jo demi-méridiens de la sphère un a jónératrices du crlindre, el renfermant chaeune les mèmes organes. On est convenu d"appeler radius les j plans rerticaus passant par te milien des antimères el qui renferment un eertain nombre dorganes importants, el intertadius les is plams intermediares renfermant d'autres opganes moins mombreux. Les ralius correspondent p. ex. aux j bras des Astéries.

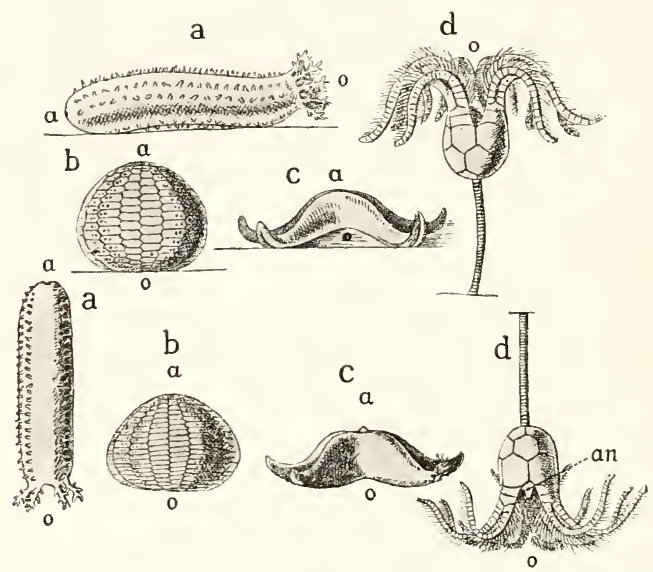

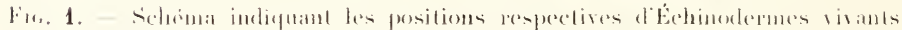

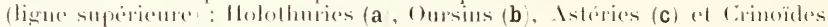
(d); cl leurn posilions morphologiques eorrespondantes (ligne infériemre); o, bouche; a, anms; liplucis Lixi.

Le squelelle apparait de tres bomne hemre chez la larve som-forme de

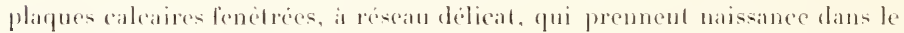

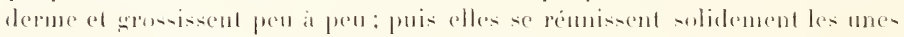

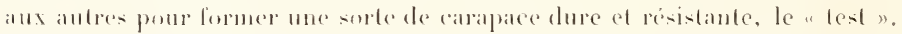

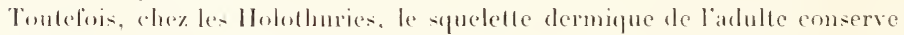
um elat embrommare; les plaques calcaires restent isoléen les mes des

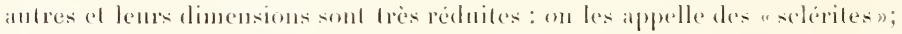
il y a cependant diverses Inolothuries chezlesquelles ces plapues devienuent assez grandes, comme ehez quelques C'ucumaria de nos mers C'. elongala, C. tergestina, p. ex. el surtoul dans le genre Psolus. Parmi les plaques du squelelte des autres belinodermes, quelques-unes se funt remarquer par lemr constance el lem arrangement régulier fig. 2 ; elle- apparaisent au 
poble opposé a la bouche el constituent un cosemble on appareil apical, qui marque le pole aboral, lequel ost généralement rlorsal, tamdis que la bouche par définition accupe le prite oral qui $r$ généralement ventral. Ces plaques dites "primaires" sont au nombre de 11 ; il y a une plaque centrale appelée centro-dorsule (c), antour de laquelle se disposent - cereles de plaques alternant entre elles : 5 sont dites radiales (r) paree quielles marquent le point de depart des plaques appartenant aux radius et 5 appelées interradiales $\mathbf{b}$ se continuent avec les plaques interradiales. Chez les Crinoïdes sypiques, te corps proprement dit est très petit et constitue une sorte de compe dont la paroi est formée presque entierement par ces 11 plaques primaires. Mais chez les autres Echinodermes, l'appareil apical ne représente

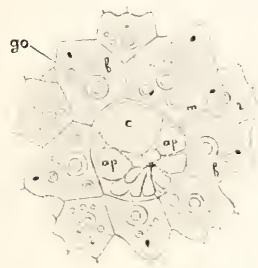

Firi. 2. - Appareil aprical d'un jeune Onrsion. c, platefue reniro-dorsale: ap, plapues du périproete: r, playues radiales printaines; b, placpues interradiales on grinitales : go, pores ginitaus diapris LOVEX).

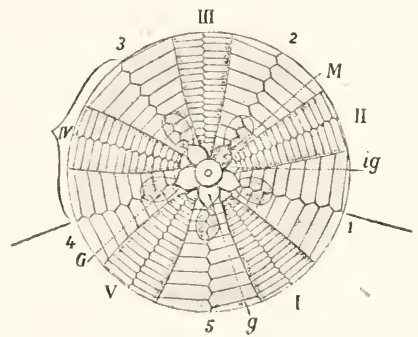

Fig. 3. Region dorsale du test din Oursin. I i V, zones ramliaim's ou ambulacrajres ; 1 à 5 , zones interradiaires; ig, placfues oce]laires; g. platpues génitales; G, glandes gennitales vues par transparence. Les deux trails obliques audessus des numeros 1 el 4 indiquent la limite entre le bivium of lo trivium: Ia partie marquece par une accolade indique un antimère (d'aplés R. Penteir).

qu'une très faible partie du squelette. Chezles Échinides (fig. 3 et 4), la paroi du corps est formée par des plaques allant de cet appareil au péristome et qui sont disposées en rangées très régulieres, 2a dans chaque radius et 2 dans chaque interradius; les premières sont souvent ippelées ambulacraires et les secondes interambulacraires. Chez les Astéries, les plaques correspondant à ces doubles rangées nexistent que sur la faec ventrale du corps: on les appelle ambulacraires et adambulacraires, et elles limitent un sillon qui part de la bouche pour śćtentre tout le long de la face ventrate de chaque bras. Il y a donc me trèstande diflérence "ut re le squelette des Astéries el celui des Échinirles. Celle dillérence est due à ce lail que chez le jeune Fohinide, il se forme, à colé de la centro-dorsale un certain nombre de plaques qui restenl petites et peu nombreuses, et qui constituent ce que l'on 
appelle le periprocte lig. 2 , lequel conserve toujours de petites dimen-

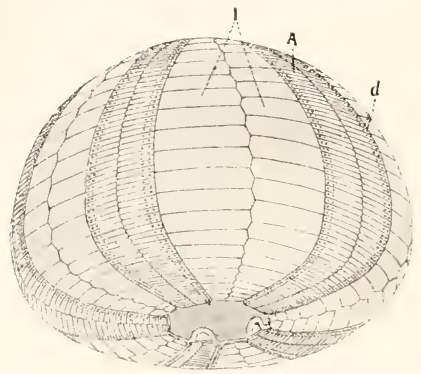

Fis. 4. - Oursin dénude, vue lationle un pen oblique. A, zone radiaire ou anbulacraire; I, zonce inlerambulacraire: $d$, joles aquifires (d'apries

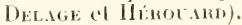
sions: aussi l'appareil apical nest pas modilié par l'intercalation de ces nouvelles plaques qui prennent en quelque rorte lieu et place de la centro-dorsale. Au comtraire, eliez les Astéries, les plaques nouvelles deviennent lres nombrenses a tres grandes: elles disloquent les plapues de lappareil apical entre lesquelles alles insinuent el quelles refoulent soil a l'extrémite des loras. soil meme sur la lace veutrale: ces momvelles plaques arrivent a constiluer ainsi presque tout le squelelle de la face dorsale du corpo fig. 5. Des modifications analogues se passent chez les Ophiures.

En ce qui concerne l'organisation interne, je ne puis guère rappeler que les dispositions londamentales de l'appareil aynifere (fig. 6 : eet appareil comprend une partie située à l’intérieur du corjs el une partie extérieure. La partie interne consiste en un amean oral entomrant la bouche on le pharvux, ef duquel partent des canaux situes an milieu des radius, lescanaux radiaires; cenx-ci lournissent de nombreux canalicules qui traversent le test et sourent dans des lubes extérieurs nombreux et serrés, les tubes umbulacraires; a la base de ces canalicules se trourent des vésicules contractiles, les vésicules ambulacruires. L'anneau oral présente jo diverticules interradiaires appelés resicules de Poli; de plus, dans un interradius, il s'en détache un canal allant souvrir à l'extérieur, le tube hydrophore, appelé souvent le "canal du sable ". Lappareil aquifère est rempli d'un liquide dout la composition est voisine de celle de l'ean de mer qui peut pénétrer lans cel apparcil: fuand les vésicules ambulacraires se contractent, elles envoient du liquide daus les lubes ambulacraires qui se gonflent, sallongent et deviennent turgescents; quand les tubes se contractent au contraire, le lıquide rentre dans l'intérieur du corps. Ces tubes sont souvent terminés par une ventouse qui, Iorxquils sont gonfless, sapplique sur le sol. les rochers, etc. : en se contractant. les tubes tirent l'Echinoderme dans une certaine direction; ils servent done i la locomotion. d'ou le nom de lubes ambulacraires. le tube hydrophore communique avec l'eau ambiante par l'intermédiaire d'une plapue, dite plaque madréporique, erense de nombreux canaux ciliés dont les orifices apparaissent a lextéricur comme autant de petits pores; cette plaque est une modification d'une plaque du squelette dont la position varie, mais qui oceupe toujours un interradius. Chez les Holothuries de uos coles. le tube hydrophore soure simplement dans la 
cavité générale. Les tubes ambulacraires de la premiere paire offrent ordinairement des caractères particuliers: chez les Holothmries, ils se développent beaucoup, acquièrent des ramifications et forment une couronne de tentacules qui entourent la bonclie.

Entre la paroi externe calcifice du corps et les organes internes se troure

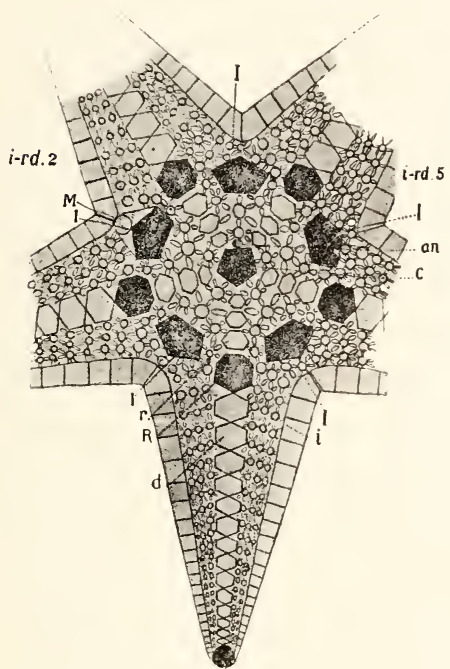

Fir. 5. - Figure sehrimatipue dus squelette dorsal d'une Astérie. $R$, plaques radiaires primaires: I, plaques interradiales primaires; $\mathbf{M}$, platues marginales dorsales: d, plaques radiaires secondaires: i, plarpues interradiaires secondaires: $\mathbf{C}$, placpue centro-dorsale; an, anu- (d'après Lı wwis).

tube digestif des Echinides (fig. 7, des Crinoïles et des Holothuries fig. 8 est cylindrique et allongé et il décrit des circonvolutions. Chez les Astéries fir, $\mathbf{9}$ et 10 et les Ophimres an contraire, c'est un simple sac qui necupe la presque totalité du disque et qui envoie, chez les Astéries, des prolongements dan* les bras. La disposition du systéme nerseur e-t calquée sur celle de l'appareil aquifère : un cercle oral et des prolongements radiaires desquels partent de fins rameaux traversant le lest et se terminant dans les appendices divers de celui-ci. Ln autre appareil, l'appareil plastidogèue, donne naissance aux cellules du liquide de la cavilé générale: il comprend encore la cavilé générale, qui est remplie par un liquide dans lequel on trouve en suspension de nombreus éléments cellulaires. Je serai très bref en ce qui concerne ces organes internes. Le

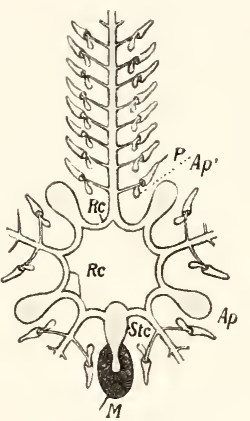

Fis. 6. - Schéma die lappareil aquifiere: Rc, cerele oral ; Ap, résicule de Poli: Stc, lube hidrophore: $\mathbf{M}$. plaque madréporique: $\mathbf{P}$. tubes ambulacraires preuant naissance sur les branches latérales des canaux acpuifères radiaires et i la base desquels se trouvent les résieules amlulacraires Ap . une cavité plus ou moins vaste, 
un cercle oral, des prolongements radiaires et surtoul un organe glandulaire accolé au tube hydrophore. Les glandes génitales occupent une siluation interradiale el sont au nombre de 5 ou de 5 pares, mais elles offrent de nombreuses variations; leurs orifices sont généralement situés sur la liace dorsale du corps. Lessexes sont presque loujours séparés.

Le lest preut porter des appendices divers qui présentent. pour la clasifi-

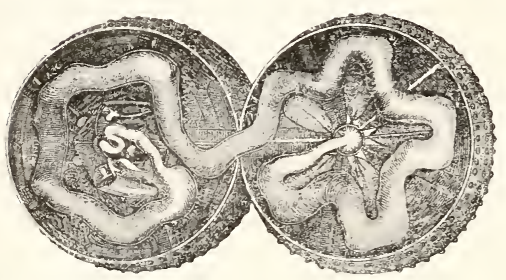

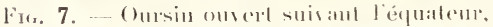
les deux moilies rabaltues de part el d'antre pour montrer le lube digestif (d'après TrDemivis.

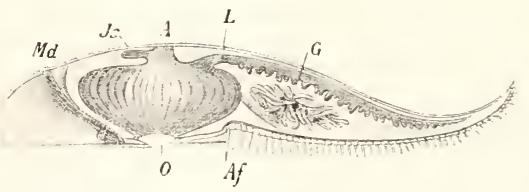

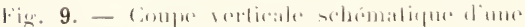

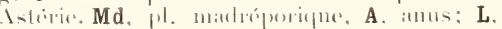

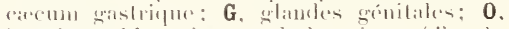
bouclie; Af, lubes aublulacratres (of appes R. PEkHIM).

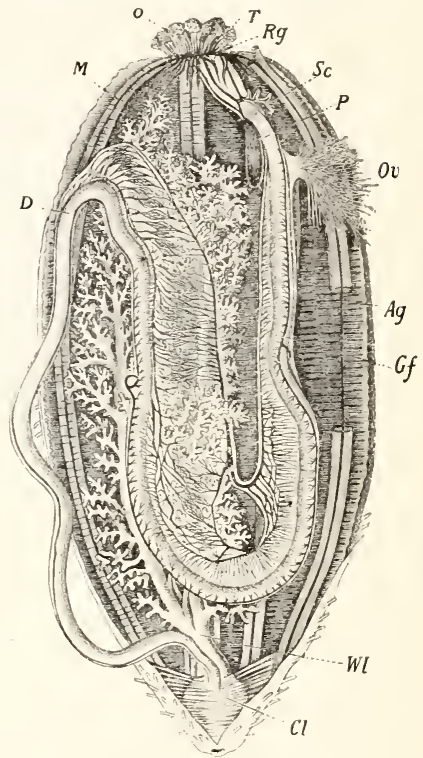

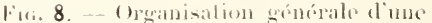
IIolothurie: T, tentarenles: o, bouche (an contre des tentac.): $\mathbf{R g}$, cerele anuition: M. muscles longit.; Sc. lubeshrdrophores; $\mathbf{P}$, risic, de Poli ; Ov, organes genit. : D, lube dig ; Wl, Irone conmun des ore. arborescents; Cl. cloatue: Ag, canal aquifère rad,; Gf, lacune intestinale (d'ap)rès MILXE EDWMDs).

cation et la détermination des Échinodermes, me frès grande importance. Ce sont dabord des pripuanls, e. a d. des baguelter calcaires on de petiles liges evlindriquen ou coniques, qui parlois ne somt quim simple prolongement diune platur, mais qui, le plus souvent, sarticulent sur un mamelon ou tuberenle de celle-ci, ef deviement moliles grice it un manchon conjonetif el musculaire reliant la tète du piquant ì ce tubercule. 
Les pédicellaires sont de petitsorganes en lorme de pinces, qui, sons heur forme la plus simple, eonsistent en deux petits piquants dressés parallèlement l’un à l'autre, et pouvant se rapprocher ou sécarter fig. 11 : des muscles spéciaux permettent ces mourements. Les pédicellates oflrent les formes les plus diverses: p. ex, au lien de pester droits, les piquants ou "valves"

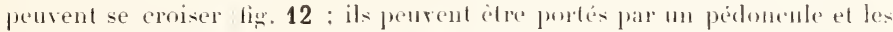

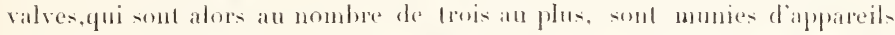
musculatires plus ou moins eompliqués, Ces petils oryanes snut surtout très développés et montrent des formes très variées chez les fichinides, et ils fournissent d'excellents caractères pour la délermination. Les pédicellaires peuvent satisil de petits corps étrangers et certains d'entre eux sont pourvus diun appareil glandulaire sécrétant un venin qui paralyse les pelits animaux fig. 14, mais leur role est eneore assez obseur.

Ifin de domner aux Echinodermes une position qui permetle de comparer entre eux les différents nroupes et de repérer les j ratius el les j interradius, on est convenu de les placer la bouche en bas, et linterradius yui porte la plaque madréporique, lorsque eelle-ci existe, en avant et i droite. Aussi la position " morphologique " des Éhino-

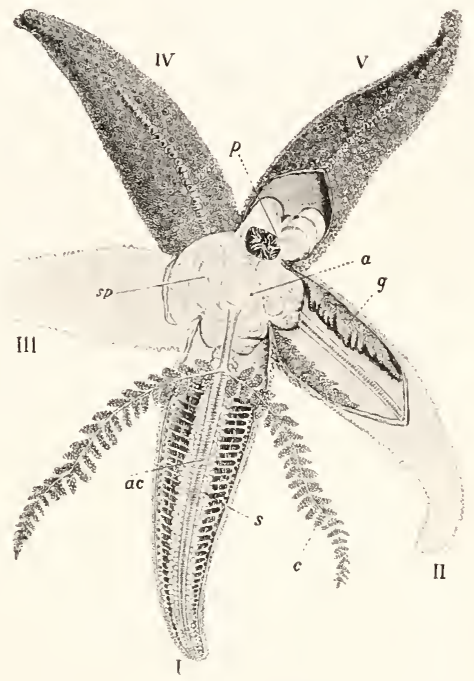

Fig. 10. - Organisation ginérale diune Istirie. sp, sac stomacal; c, cecum gastrique: a, anus: g, enlandes geinitales: s, visicule ambuaclraire; ac, canal aquifire ratiaire: p. platue madréporique (d'apres (iondrich). dermes nest-elle pas toujours la mème que dans la nature el lesschemas de la fiz. 1 permettront de comparer les représentants des principales classes placees respectivenent dans la position naturelle et dans la position morploologique. Hans ces conditions, il y a un radius antérieur, 2 rad.latéro-antérieurs et 2 rad.latéro-postérieurs d'une part : 2 interrad. latéro-antérieurs (celui de droite portant la plaque madréporique a interr. latéro-postérieurset un interrad. postérieur fig. 3 . D’autre part, Lovex a proposé de désigner le rad. post. droit par le chiffre romain I et de numéroter les suivants en sens inverse du momvement des aiguilles d'une montre: II - HI - I e l, l'animal étaut toujours placé 
sur son coté oral; les interrad. complés dans le même sens sont désignés par les chiffres arabes $1-2-3-1$ et 5 . Suivant cette nomenclature, c'est le rad. III qui est en avant et l'intermad. j en arrière; l'interrad. 2 porte la plaque madréporique. I.es rad. II, III et IV constituent ensemble le trivinm: les 2 autres. I el $\mathrm{V}$. parfois difrérents des premiers, forment le bivilum fig. 3 .

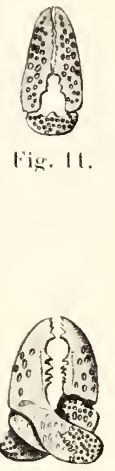

Fig. 12.

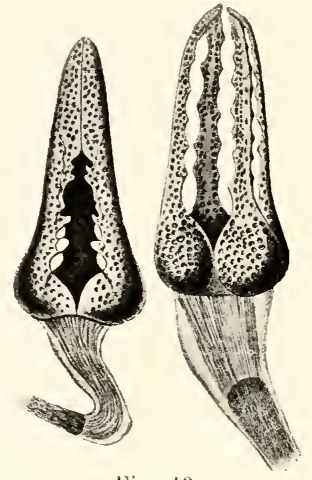

Fig. 13.

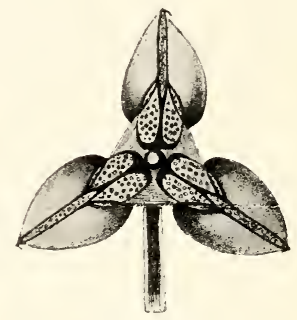

Fig. 1'.

Frti. 11. - Pédicellaire droit d'Asterias rubens (d'après Penrien).

Fur. 12. - Pédicellaire croisé d'Asterius rubens (d'après Penrier).

Fig. 13. - Pédicellaire tridactsle te Brissus unicolor (a) et tétradactyles de Schizaster canaliferus (b) (d'après Kómuen).

Fıg. 14. - Pédicellaire globifère d'Echinus acutus vus de face (d'après KoznLER).

\section{2- EMBRYOLOGIE}

Le développement des Echinodernes est très eompliqué. Lieul lécondé dome nassance a me lanve pélagique qui subit me métamorphose las complexe pour se transformer en Échnoderne : es larves présentent des formes tres particulieres a sont tres diflérentes de l'adulte. On leur a domé autrefois des noms particuliers les larves des Échinides et des Ophiures ont été appelées Pluteus, eelles des Istéries Bipinnaria et Brachiolaria, celles des Holothuries I uricularia. Le peu d'espace dont je dispose iei m’nterdit de décribe ces larves et je me contenterai seulenent d'en domner quelques dessins tign. 15 : il est d'ailleurs actuellement très difficile de rapporter telle forme larvaire it telle espèce adulte; èest une question à l'étude. 


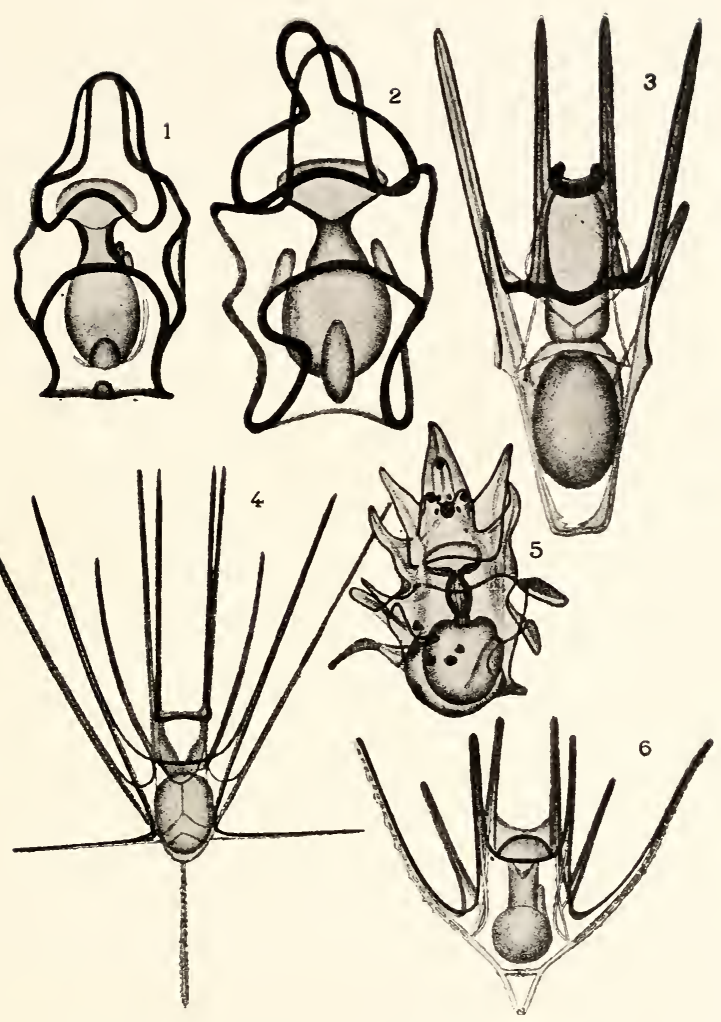

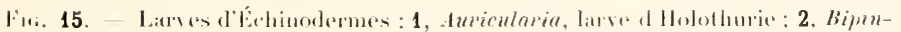

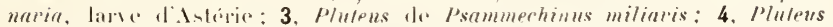

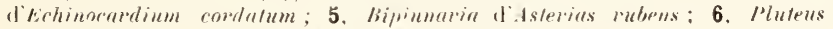
d'Ophiura lacerlosa (thaprès Yontrinev). Trés grossic.

\section{3" ÉTHOLOGIE et FAUNISTIQUE}

l.es Échinodermes vivent exelusivement don mer: on ne commat pas me seule espèce qui ail péuétré en ean doued el mème ils supportent trés difticilement une diminution dans la salinité de l'eau : éest pour cette raison qu'ils manquent dans les mers peu salées; la seule espèce qui pénètre assez. loin dans la mer Baltique est l'Asterias rubens. Quelques espèces vivent 
dans les élangs de un crites méridionales qui ammuniquent avee la mer: Gor ruet a signalé dans l'étang de Berre, les Paracentrolus lividus, Amphiura squamata, Asterina gibbosa, Astropecten plalyacanthus el Ophiura lacertosa. auxquels il faul ajouter l'a mphiura mediteranea que j"ai reneontrée récemment dans cel clang. Ces especes ne se trouvent d'ailleurs que daus les eaux les phus salées qui marquent 2 a 3 dequés Baumé. Jajoulerai encore que les lehiundermes vivent à loms les niveanx de la mer, depuis les régions qui décourent à toules les marées jusquanx plus grandes profondeurs.

Surles cotes de france, et en prenaut comme limites, vers le large, celles du plateau continental, on comple une centaine d'especes d'Échinodermes. exactement cent six, qui se répartissent ainsi 1 :

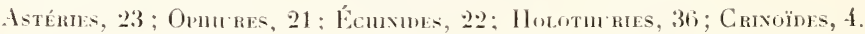
¿n petit nombre de ces espèces comportent quelques variélés.

Il faut reconnaitre que nos renseignements sur la laune échinologique sont encore très insulfisants, surtoul en ce qui concerne les cotes de l'Ucéan et il nexisle que quelques localités sur lesquelles nous possédons des indieations assez précises : Roscoff. L.e l'ouliguen, Concarueau, la Rochelle, Arcachon, Biarritz, mais les stations internédiares nout pour ansi dire pas été explorées. De plus, daus les listes qui onl élé domnces, eombien d'erreurs sont évidentes el combien d’autres pourront étre relevées. Je n'insiste pas...

J'ai tenu a accompagnes les tres courtes descriptions que je donne dans les pages suivautes de nombreuses photographies. La détermination des Échinodermes offre, pour la plupart des zoologistes, d'assez frosses difficultés, dues en grande partie à l'inexactilude des descriptions el au manque de figures. Jaurais roulu développer davantage liblustration de ce live, jaurais vouln aussi domner des descriptions complètes de nos espèces françaises, mais jétais étroltement reufermé dans les limites qui m’étaient imposées. Vajonterai que je possede dans ma collection toules les espèces que je décris. ¿ l'exception diune senle, le Psendocucumis marioni qüil ma áté impossible d'avoir: je puis dome garantir l'esactilude de mes descriptions et de mes pholographies.

\section{' CONSEILS POUR LA CAPTURE DES ÉCHINODERMES}

Le naluraliste qui recherehe les Échiusdermes ponrar en eaplurer un

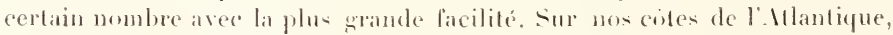

1. J'ai cru devoir introduire dans cette liste quelques esprecs qui n’ont pas encore tete rencontress sur nos côtes, mais qui, fitant connues sur les cotes d'Italie et d'Anpleterre p. ex., seront eertainement trouvees un jour dans nos mers; d'autres nont encore éte signalies que sur nos cotes d'Algérie on de Tunisic: je les ai iralement mentionnes. Toutes sont marquees d'un astérisque. 
il reneontrera ì mer basse diverses espees, les unes parmi les dyones, les autres sous les pierres, contre les wohers, elce, p. ex: Paracentrolus lividus, Psammerhinus miliaris, Asterina gibbosa, Isterias rubens, Marthasterias glarialis, Ilenricia sangumolenta, Echinuster sepositus, Astroperten irregularis, (phiothri, fragilis, Amphipholis squamalu, Amphiura filiformis, A. chiujei, Ophiocomina nigra, C'ucumaria montagui, C. leferrei, C. Lacten, C'. brumnea, Thyone roscovitu, Th, raphanus, Pseudocurumis mixlu, Ilolothuriu forsliali, Il. Iubulosa. Intedon bifidu.

Sur les plages, daus le sable pur on vasems quil lomilleral a la pioche, il rencontrera : Echinocardium cordatum, parfois E. pennalifilume el Spalungus purpurens, Ophiocentrus brachialus, C'ncumaria elongatu, Leplos!nnapta inhirems, L. galliennei, Labidopla.x digitula, L. thomsoni. Souvent la présence d'un animal est indiquée par une petite éminence de sable.

En Méditerranée, en raclant les parois des quats on des jetées, en cherchant parmi les llgues, il pomra obtenir diverses especes communes, telles que : Paracentrotus lividus, Psammechinus mirotuberiulatus, Asterina gibbosa, Coscinasterias lenuispina, Ophiomy ta pentagona, Amphima filiformis, A. chicjei, A. mediterranen, Amphipholis squamata, Ophiothri.r fragilis, Ophiomy.xa pentagona, Iniedon mediterranea.

Par temps calme, il verra sm le foud de la mer, a ㄴ-4 m. de profondeur, entre le rivage el le commeneement des prairies de Zostieses, et il pourra capturer i l'aide d'un gritppin ou d'une came i Oursins, les espèces suivantes: Paracentrotus lividus, Spharechimus sramularis, Mathas. terias glacialis, Echimaster sepositus, Astropecten spinulosus, A.jonstoni, A. bispinosus, Holothuria impatiens, II. Iubulosa, II. polii, II. forskali.

šil posiede des appareils de dragage et sil pent draguer tui-même, il recueillera la plupart des espéces de nos cotes virant a une certaine profondeur, sinon il sadressera ans pecheurs qui meltront de cold, pour hui,

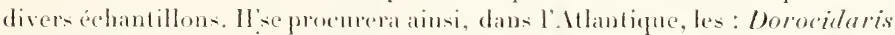
papillata, Echinus esculentus, E. aculus, Spatungus purpureus, Brissopsis lyrifera, Erhinocyamus pusillus, Anseropodu membranarea, Porania pulvillus, Soluster papposus, Stichaslrella rosea, Astropecten

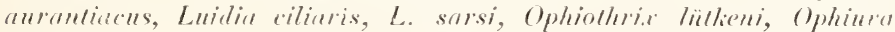
lacertosa, O. albida, Ophiactis balli, Thyoue fusus, Th. raphanus, C'ucumaria hyndmuni, Stichopus regalis, Psendormentis mixta, Leptometra cellica, des simaptes.

En Méditernané les especes sont encore plus mombrenses: Dorocidaris

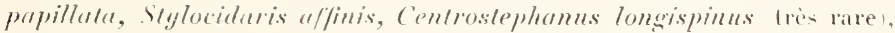
Genocidaris maculalu, lichimus aculus, E. melo, Spatangus purpureus, Echinocurdium flasescens, E. medilerranenm, E. mortenseni, Dirissus unicolor, Schizaster fragilis, Brissopsis lyrifera, Echinocyamus pusillus, 
Anseropoda membranacer, Chetaster longipes, Ilacelur attennata, Luidia ciliaris, L. sarsi, Astroperten aurantiacus, A. irregularis var. pentacanthus, Tethyaster subinermis. Astrospartus arborescens, Ophioconis forbesi, Ophiopsila aranea, O. annulosa, Ophiucantha spinulosa, Ophiura albida, O. lacertosa, Ophiothrix quinquemaculata, plusieurs C'ncumaria (planci, tergestina, firschbergi), Thyone inermis, Phyllo. phorus urna, Stichopus regalis, Lapidopla.t digitata, Leptometra phalangium. Après les tempêtes il rencontrera, rejetées à la cote, diverses formes du large.

\section{$\because$ MÉTHODES DE CONSERVATION}

Les Echinodermes, une fois capturés, ne devront jamais ètre desséchés tels quels, sous peine d'obtenir de mauvais spécimens comme ceux qu'on voit encore dans certains musées, et sil'on veut les dessécher, on devra préalablement les laisser un jour ou deux dans l’alcool. Je déconseille fortement l'emploi du formol qui dissont à la longue les corpuseules calcaires; à la rigueur, on peut l'utiliser à la condition de u'y laisser séjourner les échantillons que très peu de temps. Les Oursins, les Comatuless et les Astéries peuvent être plongés directement daus laalcool; on commenceras si l'on veut par de l’aleool a $70^{\circ}$, qui sera remplacé très lentement par de l'alcool a $90^{\circ}$. Beaucoup d'Ophiures brisent leurs bras en plusieurs morceaux quand on les plonge directement dans l'alcool, surtont les Ophiothrix, les Ophiopsila, etc. ; on devra préalablement les luer par immersion dans l'eau douce, ou encore dans l'eau de mer a laquelle on ajontera de la cocaine par petites quantités.

Les Holothuries exigent des préparations particulières. Lorsque ces animaux sont capturés, ils rejettent par l'anus une grande quantité d'eau contenue dans leurs organes arborescents, et, en même temps qü̈ls se contractent fortement, ils rétractent leurs tentacules; les Dendrochirotes rejettent en outre leurs viscères par l'anus. Pour conserver les. Ispidochirotes en extension, il laut empecher cette sortie par l'anus de l'eau et des organes internes, koit en pinçant fortement l'amms, soit en le bouchant à l'aide d'un cylindre de bois. Pour fixer l'animal en extension, on pourra employer deux movens:apros avoir serré fortement l'anus, on plongera brusquement l'échanlillon bien épanoui dans de l'eau bouillante pendant quelques secondes, et ensuite on le placera dans l'alcool; ou bien, saisistant l'animal, on enfoncera dans l'anus la canule suffisamment grosse d'une seringue remplie d'alconl et on injectera vivement une certaine quantité de cet alcool en mème temps qu'on plongera l'échantillon dans le liquide ; eette opération doit etre très vivement eomblute.

J'indiquerai a propos de chaque classe les points sur lesquels le zoologiste devar porter spécialement son altention pour déterminer ses échantillons. 


\section{EMBRANCHEMEN'T}

\section{J)ES \\ ÉCHINODERMES}

\section{TABLEAI IDES CLASSES}

Les Échinodermes se divisent en cinq elasses quion distinguera aus caractères suivants:

1. Animan exlindriques, vermiformes, ordin. mons el contractiles. sans squel. ext. diflérencié, les tég. renfermant seulement des dépoits calcaires isolés: a l'étal vivant, ils se dirigent la bouche en avant . . . . . . . . . Molothurides. p. 1 ín

- Animaux pourvus a l'état adulte d'un squel. ext. constitué par des pl. contiguës, soit soudées pour former un tout solide, soit articulées pour former des bras mobiles.

2. Le corps pr, dit est petit el a la forme diune coupe on d'un cône calice ; il se prolonge par ses bords en 10 bras formés d'art. successif: dont chacun fommit une pinnule lat, dans laquelle se développent les org. génit. : le sommet arrondi du còne est limité par une pl. e.-dors. portant des cirres formés d'art. et servant à la locomotion; les parois du calice sont limitées par un très petit nombre de pl. appartenant aux premiers articles brachiaux, et la base est fermée par me membrane offrant la bouche en son centre et l'anus excentrique porté par un tube saillant. L'animal vivant se fixe on marche a laide de ses cirres la lace orale en haut 1

(1) Certains de ces caractères ne s'appliquent qu'aux Crinoïdes de nos cìtes. Les Crinoides typiłues sont fixés à l'aide d'un pédoncule qui part de la pl. c.--dors. et le nombre des bras peut varier; les parois du calice sont formées par 5 pl. rad, et 5 interrad. Les espèces qui, comme celles de nos cótes, appartiennent a la famille des Comatulides. sont fixées pendant leur jeune àge, mais elles abandonnent de trés bonne heuroleur pédoncule pour derenir libres. 
- La lace arale est dirigée vers le has. Le corpo a-t limile par un squel. eomprenant un tris grand nombre de pl.: les whindes génit.. au nomb. de jou de jpares au plur, sont renfermées dans lintérieur du corple

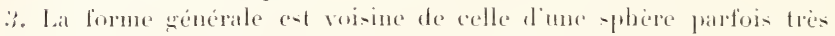
surbaissé, ou dion disque arrondi on walaire el il ny a pas de bras. Le corps est couvert de piq.: les tubes ambul. existent sur presque toute la surf. du corps . . . . Echinides. p.

- Forme stellec: le corps comprend me partice centrale, le disque, duquel partent joras plus ou moins allongés: parfois les bras sont racentreis a tel point que le corps devient un pentagone donl les angles représentent des bras rudimentaires; les tubes ambul. nexistent que sur la lace rentr.

2. La face ventr. oflre 5 sillons longil. sétentant de la bouche à l'extrém. des bras et desquels sortent les tubes ambul. disposés en 2 on en i rangées: les bras se rejoignent a leur base pour limiter le disque tont les reg. interrad. ne sont pas libres. Les bras sont creux et ils renferment à la fois des prolongements de l’appareil dig. et les grlandes génit., celles-ci sonvrant sur la face dors. 1). Il n'y a pas de pl. specialenent diffe-

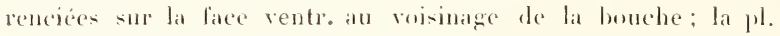
madrép, est siluéc sur la lace dors. : il existe tres souvent des pédic. . . . . . . . Stellevides p.

- Les bras sont lout a fait distincts du disque dont les réz. interrad. restent libres entre les bases de eeux-ci; le tube digr. et les glandes génit. sont localisés dans le disque et ces dernières sourrent sur la face ventr. i l'aide de lll fentes allongées, de chaque coite de la base des bras. Ceux-ci sont pleins ef comstitues par de grosses pieces calcaires articulies, appeleses rerlebres, recouvertes chacune par í pl. minces, les pl. brachiales: il existe une rangée de pl. brach. dors., une rangée de $p$ l. ventr.et 2 rangées de pl. lat. Les pl. lat. portent des piq. : les pl. ventr. recouvent les silloms ambul. el les tubes ambul. sortent lat. Lutour de la bouche, on remarque quelques pl. de forme particuliere domt liune porte le pore madrepe : les pédic. font difaut.

(1phiurides. p. is

(1) Excepté chez tes cortaines formes telle que notre Asterina gibbosa. 


\title{
GL. STELLERIIIES
}

\author{
(Astéries)
}

2 Nous savons que les Astéries sont caractérices par l’aplatissement du corps et par le grand allongement des rad. par rapport aux interrat. : il en résulte que le eor pre prend la forme d'une etoile à 5 branches, el cellesci, appelées les bras, correspondent aux rad. ; ces bras se réunissent en un disque central portant la bouche sur sa lace ventr. Les brats sont plus ou moins longs: ils peuvent dépasser lo fois le rayon du disque, (fig. 27, p. ex.) ou, au contraire, elre tellement eourts que le corps devient pentagonal (fig. 31 . Si l'on appelle $R$ la long. des bras comptée depuis la bouche, et $r$ le rayon du disque, le rapport $R, r$ varie depuis 1, 2, jusqu à 10 ou 15 .

Les 5 silloms ambul. qui śétendent de la bouche a l'extrém. des bras, out les bords limités par a séries de pl., les pl. ambul. el les pl. ardambul., ces

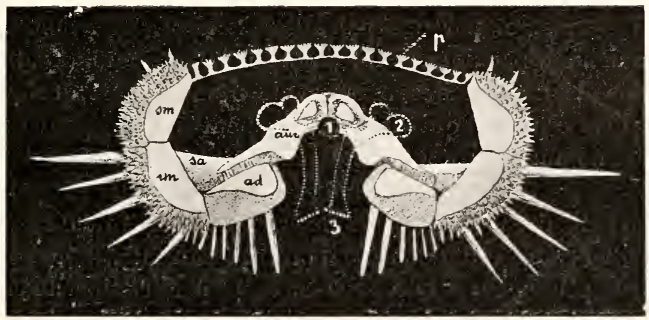

Fig. 16. Coupe transversale schrmatique d'un bras d'astropecten. sm, plapues marginales torsales: im, marginales rentrales: ad, adambulacraires:

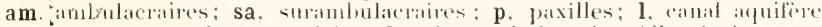

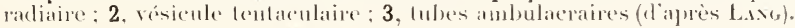

dernieres superdicielles portent des piq. dont lis disposition a me grande importance dans la elassification (fig. 16 .

Lat face dors., qui est homologue au périprocte des Échinides, est constituée par despl. nombreuses recouvertes par letég. (fig. 5 et disposées tantòt en un réseau irrégr., tantôt en rangées longit.; elles portent des piq. ou des gramules el souvent des pédic. L'mue de ees pl., plus grande que les autres el situce dans un interrad., est creusée de sillons auxquels aboulit le 
tube hydroph. : cest la pl. madrep. Entre les pl. - e trouvent des orif. par lesquels passent de petits lubes. prolongenents de la cavité générale appelés les papules el servant a la respiralion. Dans un grand nombre d'Asteries les pl. qui limitent les bords des bras se font remarquer par leur grancle taille: elles forment ¿ rangeses distinctes appeles murginales, qui sétendent sur toute la long. des bras; il y a une rangée de marg. dorsales et me rang. de marg. ventrales (fig. 16 .

Les piq. des Astéries sont de formes tris diverses : les uns sont articulés sur destubere dislincts, les autres sont fixes : ils sont moins développés que che\% les Échinides. I.es pédic, sont gènéral. constitués par 2 valves ; chez les uns, ces valves sont coroisés fig. 12 , chez dautres elles restent parallèles liune a l'autre tig. 11 ; elles sarticulent ordin. sur une piece basilaire. Tantol ces valves sont hantes el allongees, tantot au contraire elles sont basses. élargies transvers. el logées dans une petite dépression du test : dans ce cas les pédic. sout dits valpulaires. Il existe d'ailleurs d'autres formes encore. Les tég. du corps renferment des glandes sécretant un mucus qui est venimenx pour de petits animaux auquels il peut être inoculé par les morsures des pédic.

L'anus, quand il existe, se trouve au centre de la face dors. : il ne sert pas a la sortie des substances non digérées que les Astéries rejeltent habit. par la bouche.

l.es bras sout creux; ils sont en grande partie oceupes chacun par une paire de excums allongés el ramifiés sönsérant sur le sac dipestif qui remplit la cavité du disque, et par une paire de glandes génit. dont les canaux s'ourrent au dehors, par 5 pores interrad. sur la face dors. lig. 9 et 10 .

lin principe, le nombre des bras est de 5, mais il est quelquefois plus élevé. Certaines espèces, qui ont normalement 5 bras, peuvent en avoir davantage d'une manière tout à fait exceptionnelle : ainsi les Asterias rubens el Echinaster seposilus, communes sur nos coltes, ont parfois fo bras et même plus: d’autres espèces ont normalement plus de jo bas : anos le Solusler papposus en a une douzaine, ordin. égaux (tig. 26. Il arrive ansi que certaines espèces peuvent diviser leur corps par scissifarité en 2 moiliés dont chacune régénère les bras manquants. mais dans ce cas les bras régénérés ne sont pas en nombre constant, ot d'autre part, ils restent pendant longtemps plus petits que les autres ; c'est ce qui arrive p. ex. chez. la Coscinasterias tenuispina fig. 19.

Les anfis rejetés dans la mer se transforment en une larve pélagique très compliquée fig. 15. : et 5. Mais chezla petite Asterina gibbosa de nos cotes dont les orif, génit. sout placés par exception sur la face ventr., les arufs asse\% volumineux se développent directement.

Les lstéries sont fréquentes sur nos cotes. Elles so liemuent tomjours sur leur lace ventr. qui est appliquée contre les rochers, le sol, elc, ; les bras sont ordin. mobiles et souples : ils peuvent s'infléchir et se contourner en tous sens. Ja locomotion s'effectue surtout à l'aide des tubes ambul. qui se fixent anx eorps étrangers et tirent l'animal dans la direstion roulue: du 
moins chez les espèces dont les tubes sont terminés par des vent.; chez les autres, ce sont les mourements des bras qui interviement.

Les Astéries sont extrem. voraces et vivent de proies vivantes ou mortes. Elles capturent et avalent les aliments par un procédé très particulier : elles dévaginent leur sac stomacal et en enveloppent complèt. leur proie sur laquelle s'exerce l'action des sucs digestil's; tantòt la digestion a lieu dans le sac stomacal dévaginé, tantòt le sac se rétracte et rentre dans le corps avec la proie saisie. On peut ainsi trouver dans le sac stomacal des Astéries des animaux volumineux pourvus de piquants: Mollusques, Oursins, etc. L'Asterias rubens exerce de grands ravages sur nos cotes dans les parcs à Huitres : pour capturer ces dernières, elle recourbe son corps sur les deux valves et tire en sens inverse sur ces valves à l'aide de ses tubes ambul. ; l'Iluitre, obligée de céder à ces tractions, finit par s'ouvrir, et à ce moment l'Astérie évagine son sac stomacal qui pénètre entre les valres, englobe l'Iluitre et la digère.

La détermination des Astéries est en général, assez facile. L'examen ext. du corps fournit de suite des renseignements importants : le nombre des bras, la forme stellée ou pentagonale, la disposition des tubes ambul. en 2 ou en Í rangées, l'état des bras qui sont cylindriques ou aplatis, le développement des pl. marg., le recouvrement des pl., etc., permettent immédiatement de localiser les recherches. On devra souvent employer un traitement à la potasse bouillante pour étudier la disposition des pl, du squel. Il y a égal. lieu d'examiner les pédic. au microscope; enfin la disposition des piq. adambul. devra être l'objet de la plus sérieuse altention.

Les Astéries ont été divisées en quatre ordres qui sont :

$1^{\circ}$ Les FORCIPULOSÉEs 1 ; : les piq. de la face dors. sont entourés par une couronne de pédic. croisés; les tubes ambul. sont ordin. quadrisériés cl ils sont terminés par des vent.

$2^{\circ}$ Les SPINULOSEES : les piq. sont petits et disposés irrégul, ; les tubes ambul. sont bisériés el terminés par une vent. : les pl. margin. sont peu développées.

$3^{\circ}$ Les VALILLOSÉES : les pl. margin. sont très grandes ; les pl. dors et ventr. disposées en rangées longit. ou obliques, sont couvertes de granules et portent ordin. des pédic. valvulaires; les tubes ambul. sont terminés par une vent.

(1) Yote de la Direction de l'office de Funnistique : I. le professeur Kohler veut bien, à notre demande, donner à ces noms d'ordres des désinences françaises an lieu de la forme latine employée jusqu'à ce jour. En effet, les noms français sont employés pour les autres ordres et sous-ordres de l'ouvrage, et nous avons l'intention, dans toute la Faune de France, de nous en servir pour toutes les catégories systematiques supéricures da la famille (pour celle-ci, la forme à employer est, comme pour le genre et l'espèce, prescrite par les Rigles internationales de Nonenclature). 
4o Et enfin les PAXILLOSEES : les pl. de la face dors. saillantes, portent chacune un faisceau de petits piq. très serrés (paxilles); les marg. sont trés dével.; les tubes ambul. coniques n'ont pas de vent., ou ne possèdent quine vent. rudim.

\section{TABLE.IU DES ESPECES}

1. Corps pentagonal, à cités doits on un pulucxavés, ne se continuant pas en bras rraiment dilférenciés et allongés.

- Corps comprenant une rég. centr. on disque, de laquelle partent de véritables bras plus ou moins lomers, mais toujours bien distinets. ;

$\therefore$ Corps tout à fait aplati, réduit conme épaisseur à celle d'une simple fenille de carton, convert de petits pir. sur les 2. firces.

Anserepoda membramarea p. 33

- Corps offrant une certaine épaisscur.

3. Corps presque exact. pentagonal avec des cistés droits ou à peine incurvés, non amincis sur les bords, mais limités par une bordure de pl. marg. dors et ventr. grandes et ipaisses.

C'eramaster placenta p. ${ }_{\alpha} \mathbf{1} 2$

- Les cotés du corps sont plus ou moins excavés, les bords sont très amineis, et les pl. marg. sont indistinctes ou très petites. . . . 4t

4. Faces dors. el ventr. couvertes d'un tég. épais cachant les pl. sousjacentes et dépourvues de piq.; les seuls piq. indépeudamment des piq. adaubul.) sont des piq. marg. qui s’étendent sur le bord du corps par groupes de 2 ou 3 ; diam. atteignant et pourant mème dépasser $10 \mathrm{~cm}$. . . . . . . Porania pulvillus ip.

- Faces dors. et ventr. couvertes de petits piq. serrés; le diam. ne dépasse pas 1 à $5 \mathrm{~cm}$. . . . . . . Asterina gibbosa (p. 33

5. Corps en forme de soleil comprenant un disque centr. très grand duquel partent en rayonnant me douzaine de bras périphér. courts.

Solaster papposus p. 35 )

- Corps étoilé, à bras plus ou moins allongés, nornalement au nombre de 5 . .

i. Bras arrondis ou pentagonaux, non élargis à la base, disque petit, face dors. du corps munie de granules ou de piq.

Bras aplatis, beaucoup plus larges que hauts: face dors. couverte de paxilles. . . . . . . . . . . . . . .

7. Les bras offrent en coupe la forme diun pentagone; piq. le la face dors. forts, entourés à leur base d'une collerelte de pédic. croisés: sillons ambul. très larges, tubes ambul. quadrisériés . . . . . 8

- Les bras sont arrondis ; les piq. sont petits, courts, serrés ou sont remplatée fiar des granules. 
४. Les piq. de la face dors, en génèral forts, sont disposés en rangées longit. rég. peu nombreuses, répondant à un squel. formé luimême de pl. disposées en rangées longit..

- Les piq. de la face dors. sont nombrem, petits, peu pointus, ne formant pas de rangées distinctes, saul une rangée carinale. .

Asterias rubens ip.

9. Bras en nombre sup. à jar suite d'me reproduction fissipare et général. inégaux . . . . . Coscinasterias tenuispina p.

- Bras nomalement an nombre de j.

10. Espèce littorale de grande taille, munie de piqf. très forts, coniques et pointus. . . . . . Marthasterias glacialis p.

- Espece de pelite taille et virant tomjours ì une certaine prof.; piq. adimbul. sur 2 rangs. . . . Sclerasterias guernei p.

11. Sillons ambul assez larges lubes ambul. quadrisériés : corps couvert de granules ou de petits piq. tresserrés, recourrant des pl. petites et imbriquées; des pédic. droits et croisés épars.

Stichastrella rosea p.

- Sillons ambul. étroits; tubes ambul. formant 2 rangées seulement, corps couvert de petits piq. ou de granules; pas de pédic. croisés . . . . . . . . . . . . . . . . . 1

12. Squel. formé par un réseau calc. irrég. supportant de petits piq..

- Squel. formé par des pl. disposées en rangées longit. régul. et très apparentes

13. La face dors.porte des piq. très courts ressemblant presque à des granules, ordin. réunis par groupes de 5 à 8 et ne s'articulant pas sur des mamelons distincts; des papules sur la face rentr.; couleur violacée ou rosée. . . . Henricia sanguinolenta (p.

- La face dors. porte des piq. assez déreloppés, isolés, s'articulant chacun sur un mamelon distinct; pas de papules sur la face ventr.; couleur rouge ou rouge-brique très vive .

Echinaster sepositus (p.

14. Pl. petites, carrées, égales, assez saillantes, séparées par des sillons bien marqués, formant une sorte de parage et portant des piq. extrêm. fins et vitreux; entre les pl. se trouvent de petits orifices arrondis laissant passer chacun une papule; bras très longs et très étroits

Chrotaster longipes (p.

- Pl. assez grandes, aplaties. entre lesquelles se trouvent des aires porifères, c. ¿. d. des plages de din. voisines de celles des pl. et renfermant chacune 15 à 20 papules; tout le corps est couverl de granules serrés et très fins.

15. Bras cylindriques, arrondis à l'extrém. et conservant à peu prè la même larg. sur toute leur long.; 8 rangées d'aires porifères. 
- Bras allant en se rétrécissant progress. depuis la base, et assez pointus a l'extrém. : 10 rangéen d’aires porifères.

Hacelia allennala $\mathrm{P}$.

16. Bras élargis a la base par laquelle ils passent progress. au disque qui est grand; -2 rangées de pl. marg. lèes distinctes; bras solides et résistants.

- Bras non élargis a la base, longs, assez étroits. minces et se brisant avec une très grande facilité; pas de pl. marge. dors. distinctes G. Luidia

17. 7 bras

Luidia ciliaris p. 55

- 5 bras Luidia sarsi ( $\mathrm{p}$.

18. Aires interad. ventr. grandes: pl. mary. dors. courtes, mais très larges, convertes de granules, pl. marg. ventr. assez courtes et couvertes de petits piq. dont les plus ext. apparaissent a ipeine quand on regarde l'Astérie par en haut. Telhyaster subinermis p. 54 Aires interrad, ventr. petites: les pl. marg. dors, portent ordin. un ou plusieurs petits piq. 'n plus des gramules; les marw. ventr. portent des piq. trés dével. dont les plus ext. débordent largement le corps (i. Astropecten).

19. In seul piq. adambul. int.; pl. marg. dors. portant des granules souvent allongés ef un nombre variable de petils piq. Espèce d’assez petite taille dont le diam. ne dépasse guère ś cm.; la lace dors. est d'un brun assez lincé. Astropecten spinulosus p. is

3 piq. adambul. int.

:0. Pl. marg. dors, portant, en plus des gramules, soit 1 seul, soit 2 ou 3 piq. forts el pointus. .

- l'l. marg. dors. noffrant, en phos des granules, quim seul piq. petit, court et qui peut manquer complèt.

?. Pl. marg. dons. armés chacune d’un piq. mique très dévoloppé, fort aplati et pointu; pl. mare. ventr. ordin. nues sur une bonne partie de leur surl. el portant des piq. sur leurs bords seulewent; le plus ext. de ees pir. est tris grand, aplati, sonvent tronqué à l'extrém. Lal fare dor's. est d'um brum assez foncé.

Astropecten bispinosus ip.

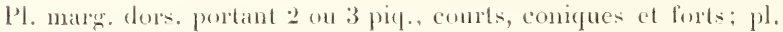
marg. ventr, couvertes de nombreux piq. dont les plus ext. sont grands el pointus.

22. Espèce de très grande taille el de coulenr orangé à l'ètal rivant; les pl. mar... dors. portent 2 on 3 piq. coniques, forts, mais assez courts; 2 piq. adambul. ext.et 3 int. Astropecten amrantiacus p. Espèce de taille moyenne: pl. marg. dors. portant 2 ou 3 piq. coniques; 3 piq. adambul, int. et 3 ext. Var. de l'A. irregularis connue surtout aux environs de La liochelle et rare 
23. Espèce de petile taille à bras courts, larges à la base el de forme triangulaire; les pl. marg. dors. portent un petil piq. qui manque sur les 3 ou í premières de chaque série; les pl. marog. ventr. n'offrent qu'une simple bordure de piq. ì leur périph.; sur le coté ext., l'un de ces piq. s’allonge beanconp ol devient très grand, aplati, avee l'extrém. tronquée, mais prespue foute la face ventr. de ces pl, reste nue . Astropecten jonstoni p. i!

- Espèce de taille moyemne ou assez grande, a bras plutist étroits, allongés: pl. nary. dors. tantot munies d'un petit piq.. tantót complèt. inermes ; pl. marg. ventr. convertes de piq. lrès serrés, qui, sur le bord aboral, s'allongent progress. surtout les 2 ou 3 plus ext.

?'. Pl. maro. dors. munies d'un petil piq., parfois de 2

Astropecten irregularis typicus ip. it

- Pl. marg. dors, inermes. A. irregularis var. pentacanthus (1. ..2,

\section{O. FORCIPLLEES}

\section{F. ASTERIIDÆ GraY.}

Les ossicules du squelelle dors. al lal. tles bras sont tantot granels el disposés en rangées longit. peu nombreuses, tantót plus petils el formant un réseau plus ou moins irregul, ; les pir. sont grands of relativement peu nombreux, ordin. entourés à la base d'ume colleretle membraneuse renfermant un grand nombre de pédie. croisés, les pédic. droils restant éprars; les aires papulaires sont grandes et laissent passer plusieurs papules a la fois; les tubes ambul. sont quadriscriés; la bouche est grande el dilatable.

\section{(i. MARTHASTERIAS JiLLIEv.}

Voir : Verrili, 1914, p. it.

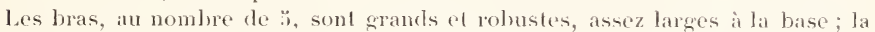
face dors. ofre une rangée longit. de pl. formant une série carin. très rég., en dehors de laupuelle se trouvent 2 ou 3 rangées lat. un peu irróx., puis, sur le cóté, une série marqu. dors. Ces pl. portent chacune un gros pir]. fort, conique, assez pointu, les pirg. lat. un peu moins forts que les autres. Iés cotés de la face ventr. sont limilés par une rangée marg., charque jl. portant 2 piq. disposés un peu obliq. l'un par rapport à l'autre; ces pị. ont la mème forme que les autres, mais ils sont général. un peu plus petits. Les piq. sont entourés à leur base par une collerette de pédic. croisés très compacte et épaisse. Des pédic. droils sont 
épars sur la face dors. ; ils sont plus nombreux sur la face ventr. et dans le sillon. Aux pédic. droits de la forme ordin. à valves triangulaires s'ajoutent quelques autres pédic. ordin. plus grands, dont les valves sont élargies en spatules à l'extrémité : ce sont des pédic. "en palette." Les piq, adambul, sont disposés sur une rangée unique et très régul,

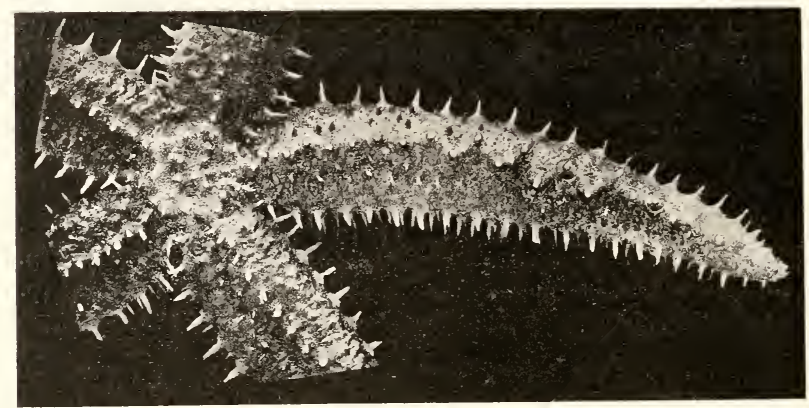

FIG. 17. Marthasterias glacialis; face dorsale: $\times 1 / 2$.

M. glacialis L. Asterias gl. anct. Fig. 17. - Voir : Bell. 1892, p. 98 ; Ledwig, 1897, p. 364, pl. III, lig. 1 à 3.

Le corps est très robuste et de très grande taille : $R=12$ à $15 \mathrm{~cm}$. et peut dépasser cette long.; dans un échant. chez lequel il a $14 \mathrm{~cm}$., les bras ont 26 à $28 \mathrm{~mm}$. de larg. à la base. Les piq. sont très forts, épais, avec la pointe tantôt aiguë, tantôt émoussée, et écartés les uns des autres; ceux de la rangée carin. forment souvent une ligne en zig-zag; les piq. lat. sont un peu plus petits et, chez les très grands échant, ils forment souvent 2 rangées plus ou moins distinctes. Les piq. des pl. marg. dors. sont un peu plus forts que les carin., mais ceux des pl. marg. ventr, au nombre de 2 par pl., sont un peu plus minces et les inf. sont plus petits que les sup. Les piq. du disque sont assez nombreux et rapprochés, beaucoup plus petits que ceux des bras. Les piq. de la face dors. sont entourés à leur base d'une très large collerette, mesurant '1 à $6 \mathrm{~mm}$. de diam., contractile et bourrée de pédic. croisés qui apparaissent à l'ceil nu comme autant de petits points clairs. Sur les piq. marg. ventr., les collerettes sont incompl. et n'existent que sur le cóté dors. Entre les piq., la face dors. est parsemée de pédic. droits.

Les échant. littoraux ont toujours une couleur très foncée qui varie du brun au vert sombre ou au vert olivâtre plus ou moins foncé; au contraire, ceux qui proviennent d'une certaine profondeur, $50 \mathrm{~m}$. p. ex., et qui sont d'ailleurs plus grands et plus trapus que les indiv. littoraux, car ils peuvent atteindre jusqu'à $40 \mathrm{~cm}$. de diam.. ont des couleurs plus vives qui varient du rose au 
rouge ou au brun acajou avec des taches blanches. Ia couleur passe dans l'alcool.

La $M$. glacialis est extrèm. répandue sur toutes nos còtes, en Méditerranée comme dans l'Atlantique; elle est surtont littorale mais elle descend fréquemment a $50 \mathrm{~m}$. de prof. Elle est peu commune dans la Ianche, mais elle se trome en de nombreuses localités de la mer du Vord, sur les cotes dingleterre et jusqu à celles de Yorrège, tandis que vers le S. elle s'étend sur les côtes d'Espagne et sur les côtes d'Afrique jusqu'aux iles du Cap V'ert; elle preut atteindre une prof. de $150 \mathrm{~m}$.

\section{G. ASTERIAS Lixié. $s, s t r$ Virrul. rest.}

Voir: Verhill, $191 t$, p. 101.

le sifuel. est constitué par des pl. irrégul. disposées en un réseau plus ou moins serré, mais ne formant pas de rangées longit. rég. Les piq. que portent ces pl. sont aussi irrégul. disposis, sauf sm la ligne carin. qui porte en général une rangée un peu sinueuse. Cés picy., conts et eylindriques, sont entourés à leur base d'une petite eollerette à pédie. croisés, et, entre eux, se montrent des aires papulaires nombreuses et irrég. Les piq. des pl. marg. dors., au nombre de 1 ou 2 par pl., forment une rangée longit. très distincte: ceux des marg. ventr. sont plus développés : ils sont au moins au nombre de 2 et parfois de 3 , constituant une petite série obl. ; ils sont séparés des premiers par un large intervalle nu. Les collerettes à pédic. de ces piq. sont ordin. incomplètes. L.es piq. adambul. sont disposés tantôt sur 1 seul rang, tantòt sur 2 et il $\mathrm{y}$ a ordin. une alternance irrég. entre pl. à 1 piq. et pl. à 2 piq. Entre ces piq. adambul. et ceux des pl.'marg. ventr. se trouvent des piq. ventr. formant tantôt 1 seule, tantòt 2 , et parfois même 3 rangées distinctes et régul. qui correspondent à autant de pl. ventr. Entre les piq. se montrent de nombreux pédic. droits, qui deviennent plus nombreux et plus gros sur la face rentr. ; ees pédic. existent aussi sur les parois du sillon ambul. et souvent ils sont portés direet. par les piq. adambul. eux-mèmes.

A. rubens (L.). Fig. 18. - Voir : Bfll, 1891, p. 169, pl. XIV, et 1892, p. 100 ; Cú́xot, 1912 , p. 21.

L'A. rubens de nos còtes est susceptible de présenter de grandes variations qui portent à la fois sur la laille des échant., sur la grosseur des bras, sur le nombre, la forme et la disposition des piq.. et sur la coloration. Le diam. est habit. compris entre 12 et $15 \mathrm{~cm}$., mais il peut dépasser largement 20 $\mathrm{cm}$. Les bras sont tantôl relat. larges et courts, un peu rétrécis à leur insertion sur le disque, tantôt minces et allongés. Les piq. de la face dors. sont en général disposés sans ordre régul., cependant dans cerlains exemplaires et en certaines parties des bras, on trouve des indications d'alignements; ceux de la ligne carin. forment toujours une rangée longit. bien apparente, tantôt droite, tantôt sinueuse. Ces piq. sont plus rapprochés sur certains indiv. que sur d'autres; tantôt ils sont cylindriques avec la pointe arrondie et spinuleuse, tantôt ils sont nettement renflés à l'extrémité et capités. Les piq. adambul., séparés des marg. ventr. par un certain intervalle, sont un peu 
irrégul. disposés, le plus souvent au nombre de 2 par pl., mais parfois au nombre d'un seul et il y a des alternances irrég. Ces piq. présentent toujours un caractère très constant, qui n'existe chez aucune autre Astérie de nos côtes : ils portent en divers points de leur hauteur, mais surtout dans leur tiers ext., des pédic. droits, parfois nombreux, implanlés direct. sur eux. Dautres pédic. droits se montrent sur le tér. de la face ventr. el sur la paroi des sillons. Les collerelles à pédic. eroisés sont plus ou moins développées suivant les indiv. Les échant. ayant plus de st bras ne sont pas rares, on en a trouvé it 6,7 on 8 bras, el Cóxót a même cité un indiv. d'Arcachon possédant 9 bras. Les ex, ir 1 bras sont plus rares.

La couleur à l'élal vivant esl assez variable; souvent la face dors. est orangée et parfois elle prend une teinte plus claire, d'un blane jaunâtre ou jaune grisâtre: d'autres indiv. sont d'un rouge assez vif ou rouge grisitre, d'autres enfin sonl violel loncé el il existe tous les intermédiaires possibles entre ces colorations; la lace ventr, est plus claire. La couleur passe dans l'alcool.

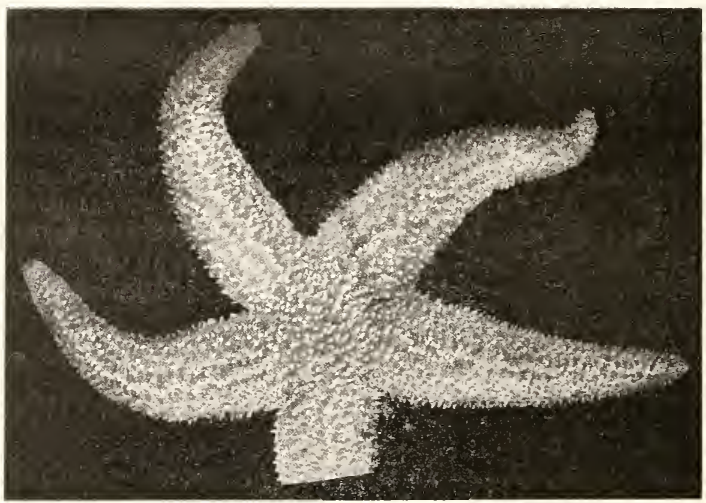

F1G. 18. - Asterias rubens, face dorsale ; $\times 1 / 2$.

L'A. rubens est une espèce lrès répandue sur nos cóles de la Manehe el de l'Atlantique. On la trouve à mer liasse sous les pierres, contre les rochers, parmi les Algues, sur le sable ; elle est tris commune dans certaines localités, tandis que dans d'autres elle fait complet, defant comme à Roscofl, liranville, ele., sans tfue l'on connaisse la raison de ces dillérences. Elle est très répandue sur les eòles d'Angleterre et remonte vers le X. jusquä la mer Blanche; comme elle tolère une eau peu salée, elle pénitre assez loin dans la mer. Baltique. Elle descend ¡usqu’au Sénégal; ses limites extrèmes en prol. sonl 0 et $200 \mathrm{~m}$.

L'A, rubens a été signaléc parfois en lléditerranée mais elle y est fort rare; 
cependant je laai trouvée très abondante à Cette, dans un pare à IIuîtres, où elle a été sans doute introduite arec ces Lamellibranches. L'A. rubens fait, en eflet, des ravages énormes dans les pares à IIuîtres et à Jloules de nos côtes occidentales. Jaai expliqué plus haut la manière dont elle ouvre les coquilles d'Huitres pour avaler le Mollusique.

\section{G. COScinasterias Verrit..}

Voir: Verrill, 1914, p. 4...

Les bras étroits sont en nombre variable et général. sup. it 7 ; il existe ordin. 2 pl. madrép. et parfois 3 . Les bras oflent une rangée carin. de piq. puis une rangée latérale, et it la suite, une rangée marg. dors. et une marg. rentr. Tontes ces rangées sont régul., el correspondent it dé pll. bien alignées. Des collerettes à pédic. existent à la base deu pirf. et des pédic. lroits se montrent épars sur la faee dors. ainsi que dans le sillon; les pit. adambul. sont disposés sur une seule rangée.

La plupart des espèces du genre Coscinasterias peuvent se multiplier par fissiparité et les ? moitiés régénèrcull les bras manquants, sourent en nombre variable ; c'est pour cetle raison que les bras sont souvent inéganx, et que leur nombre rarie de 6 ì 10 en général.

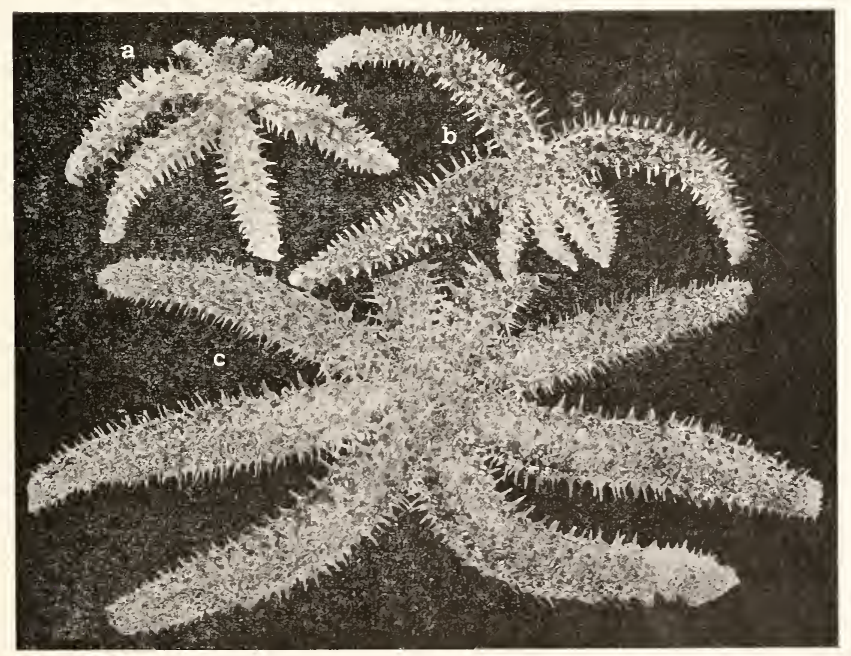

FIG. 19. - Coscinasterias tennispina; face dorsale; a, jeune il quatre grands bras et quatre petits; b, jeune it trois grands bras et quatre petils; c, atulte; $\times 1 \%$ 
C. tenuispina Lamarck [Asterias t. aucl.]. Fig. 19. - Voir : Lebwis, 1897, p. 334, fig. 43 , pl. III, fig. 8 .

Le nombre des bras rarie habit. de 6 à 9 . Lorsque l'Astérie n'est pas adulte, ces bras sont inégaux et ceux d'un còlé sont beaucoup plus petits que les autres: ils viennent d'être régénérés. Al'état adulte, les bras sont ordin. subégaux et le diam. du corps rarie alors entre 15 et $18 \mathrm{~cm}$. Les bras sont asser. élroits el les piq. sont relat. plus fin- et plus nombreux que chez la $\boldsymbol{M}$. glacialis; ils sont aussi plus régul. alignés. Il existe souvent, chez les grands exempl., ¿2 rangées de piq. lat. dors. à la base des bras. Des pédic. en palette se montrent parmi les pédic. droits ordinaires. En général, il existe 2 pl. madrép.

La couleur ì l'état vivant est assez variable. La face dors. est brunâtre ou jaune brunatre, avee des taches foncées el les piq. sont plus clairs; sur d'autres indiv., la leinte générale est d'un brun rougeatre; la face ventr. est toujours plus claire. Ces coloralions disparaissent complet. dans l'alcool.

La C. tenuispina vit surtout en Méliteranée où clle est très abondante; elle est essent. littorale et ne dipasse guère 3 à $\dot{t}$ m. de prof.; exceptionn. elle peut descendre à $10 \mathrm{~m}$. el Lrowig la mentionnne à $40 \mathrm{~m}$. 11). On la trouve à la côte, sous les pierres, associée aux Marthasterias glarialis, Asterina gibbosa, ete. En dehors de la Méditerranée, elle a été rencontrée surtout sur les côtes des îles africaines (Açores, Canaries, îles du Cap Vert); on l'a trouvée également à Setubal, sur les côtes d'Espagne et sur nos côtes du S. W., mais elle ne remonte pas dans les mers du N.

\section{G. SGlerasterias Perrier.}

Les pl. et les piq. de la face dors. forment des rangées longitud. rég. mais peu nombreuses : il existe une rangée carin., une rangée marg. dors. et une marg. ventr., enfin une rangée lat.-dors. un peu moins développée mais qui se continue néanmoins presque jusqu'à l'extrémité des bras. Ces pl. se correspondent exactement sur une même rangée transv., et forment des arceaux bien distincts séparés par des sillons transr. dı tég. qui est assez épais. Les pl. dors. portent chacune

(1) Marion a indiqué la C. tenuispina entre Marseille et la Corse à une prof. de 250 m. ; Ludwig fait remarquer à ee sujet qu'il y a certainement eu erreur de détermination et qu'il s'agissait de l'Hydrasterias richardi Perrien, qui, à l'état jeune, possède 6 bras.

Je ne mentionne pas cette dernière espèce parmi les Echinodermes français, ear elle n'a été rencontrée en Néditerranée qu'à de grandes prof., dans le golfe de Naples : Perrier l'a signalée aux fles du Cap Vert (225-540 m.). Il en est de mème de la Stylasterias neglecta Perrafa /Asterias edmundi Ludwig tronvée vers Cérigo et l'ile de Crête, entre 160 et $465 \mathrm{~m}$. Un exemplaire unique et de très petite taille $(R=15, r=3 \mathrm{~mm}$.) a été indiqué par L. Perrier, au large des Sables-d'Olonne, à une profondeur de $166 \mathrm{~m}$. La $S t$, nogleclu se reconnait à ses piq. formant 5 rangres et entourés d'une collerette à pédic. croisés, à l'absence de pédie. droits, aux piq. adambul. bisériẻs et à la présenee de papules sur la face ventr. 
un petit piq. cylindrique émoussé, entouré d'une collerette à pédie. ; les pl. marg. ventr. portent ehacune 2 piq. disposés obliq. Les sillons ambul. ne sont pas très larges et les tubes ne sont général. disposés sur 4 rangées qu'à la base des bras. Les piq. adambul, sont très régul. biscériés.

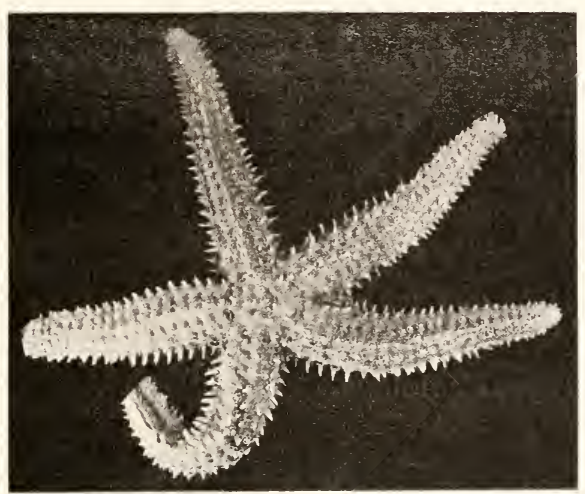

Fic. 20. - Sclerasterias guernei: face dorsale, ligèrement grossie.

S. guernei Perrier. Fig. 20. - Voir : E. Perrier, 1896, p. 33, tig. 4. pl. I, fig. 1 et $1^{\text {a }}$.

L'espèce est de petite taille et $R$ varie ordin. entre 20 et $25 \mathrm{~mm}$., il peut cependant atteindre $3 \mathbf{i} \mathrm{mm}$. La couleur à l'état vivant est d'un brun assez clair avec des lignes brunes plus foncées correspondant aux sillons qui séparent les arceaux successifs de pl. : celte couleur est en partie conservée dans l'alcool.

La $S c$. guernei vit au large de nos cotes atlantiques et it une certaine prof. Je la signale iei paree 'que je l'ai reeucillie, à bord du "Caudan ", à la limite de ce plateau, vers $190 \mathrm{~m}$, : clle descerd jusıu’a $500 \mathrm{~m}$. de prof. C'est une esp. rare.

\section{F. STICHASTERIDÆ SL.IDEN.}

Les pl. dors. et lat. du corps sont petites, nombreuses, disposées en plusieurs séries longit. et ordinairement imbriquées: elles ne portent pas de piq. mais sont recouvertes de granules serrés; les pédie. croisés sont épars et jamais réunis en collerettes autour des piq. ; les papules sont isolées. 


\section{G. STICHASTRELLA VerRill.}

Voir: Verrill, 1914 , p. 40.

Les bras sont arrondis; les sillons ambul. sont de moyenne dimension et les tubes, pourvus de vent., sont quadrisériés au moins à la base des bras. les pl. du squel. sont nombreuses, petites, très rapprochées, un peu imbripuées et assez irrégul disposées sur la face dors, mais sur les côtés des bras elles forment 2 rangées longit, assez distinctes eorrespontant à des pl. marg. dors. ot ventr. Entre les marg. ventr. et les adambul. il existe, it la base des bras, 2 et parfois 3 rangées longit. de pl. ventr. Les pl. clors. et marg. sont couvertes de granules très serrés, entre lesquels se montrent de petites papules dont la disposition irrég. correspond à la disposition irrég. des pl.; sur les côtés, les papules se disposent en séries longit. Les pl. adambul. portent chacune 2 à 3 piq. très courts, placés irrégul., et les pl. rentr. qui leur sont contiguës portent aussi des piq. lrès courts, iclent. aux piq. adambul. Les pl. earin. des bras sont un peu plus grandes et plus larges que les voisines et elles forment une rangér longit. assez distincte. Entre les granules se montrent quelques pédic. croisis isolis; des pédic, droits peu abondants existent entre les pị, adambul, et sur les cotés du sillon.

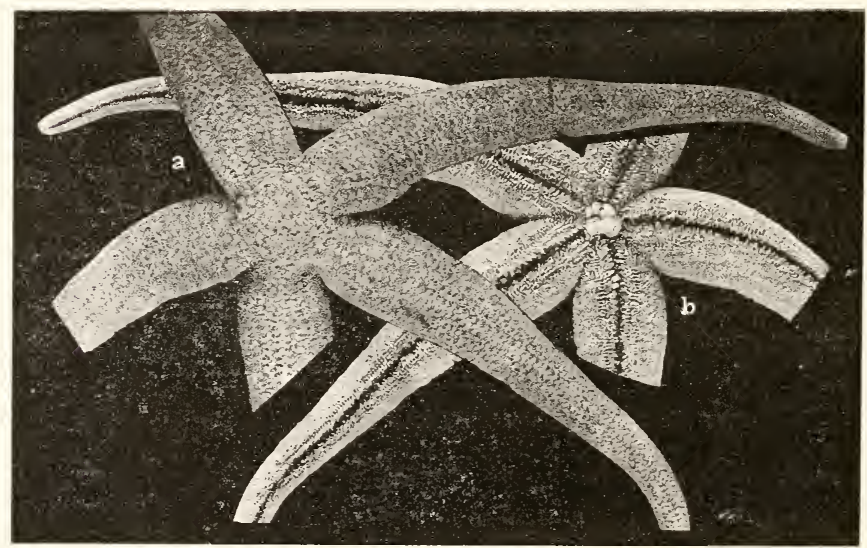

Fro. 21. - Stichastrella rosea: a, face dorsale: b. face ventrale: $\times 2 / 3$.

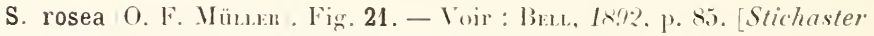
$r$ Қйек, 19?1 a, p]. XXII, lig. 6-8 et LXI, fig. 1.

I a $\$$ \% rosea a les bras très allongés, cylindriques et un disque petit; elle peut atteindre d'assez grandes dim. et $R$ varie entre 10 et $15 \mathrm{~cm}$. Elle offre à l'état vivaut une teinte rosée qui disparaît dans l'alcool. 
Cette esp. manque en Médilerrance; sur nos cotes Atlanticues, on la rencontres général. à une assez faible prof., de 20 i 30 m., mais elle peut descendre jusqü it $180 \mathrm{~m}$. Elle s'étend vers le $X$. sur les cotes d'Angleterre et jusqu'en Norvège, mais elle ne deseend pas beateoup rers le s. Ses timites extremes en prol. sont 4 et $360 \mathrm{~m}$.

\section{O. SPINLLOSEES}

\section{F. EGHINASTERIDÆ VERRILL.}

Le squel. dors. est formé d'ossicules très petits réunis en un réseau irrég. portant des pirf. très fins non groupés en paxilles; il n’y a pas de pédie.; les plaques aclanbul. portent des pir. formant une seule séric perpendic, au sillon; les bras sont allongés.

\section{G. ECHINASTER Müller et Troschel}

Les bras sont arrondis, assez longs et le disque est petit. Les ossieules de la face dor's. forment un réseau très irrég., lâche el limilant des espaces membraneux assez grands par lespuels passent plusieurs papules. De ce résean s'blérent des piep. assez conrts sirticulant sur un petit manclon et qui pensent s'infléchir latér. Sur les cotés des lmas, les pl. tendent il former des rangées longit. et l'on en distingue surtout 2 qui correspondent i des pl. marg. dors. et ventr. Les pl. ventr. peu nombreuses nexistent quä la hase des bras. 11 n'y a pas de papules sur la face ventr. lés sillons ambul. sont étroits el ils peuvent se fermer complèt. de manière à cacher les lubes ambul. qui sont disposésen 2 rangées et se terminent par une forte vent. Les pl. adambul. portent un petit piy. int. en forme de lame de sabre, et, sur leur face ventr. Z antres piq. de même taille que les piq. ventr. voisins. Les tég. renferment de nombreuses gl. muqueuses.

E. sepositus Griy. Fig. 22. - Voir : Ledwg, 1897, p. 313, pl. IV, fig. í et 5 .

Le disque est petit; les bras, arrondis, avec la face ventr. légèr. aplatie, vont en s'amincissant progress. jusqu'à l'extrémité qui est obtuse; ils peurent être très longs et atteindre jusqu'à $15 \mathrm{~cm}$., mais leur longueur est ordin. comprise entre 7 et $10 \mathrm{~cm}$. Le rappport $R r$ varie entre 6 et 8 . Les piq. petits et courts, n'ont guère plus de $1,5 \mathrm{~mm}$. de long., et ils sont enfoncés en partie dans le tég., leur extrémité est obtuse. Ces piq., quoique très rapprochés, ne sont pas réunis par groupes et ils suivent les contours du réseau squelet.; tique. Les piq. marg. sont un peu plus grands que les voisins. Les piq. adambul, sont au nombre de 3 , les 2 ext. un peu plus forts. Des indiv, à 6 et niême 7 bras ne sont pas rares. 
Lanimal vivant présente une coloration rouge très intense, tantòt rouge brique, tantit rouge orangé, plus ou moins foncée; la face rentr. est un peu plus claire que la face dors. Ia eoloration disparait complet. dans l’alcool.

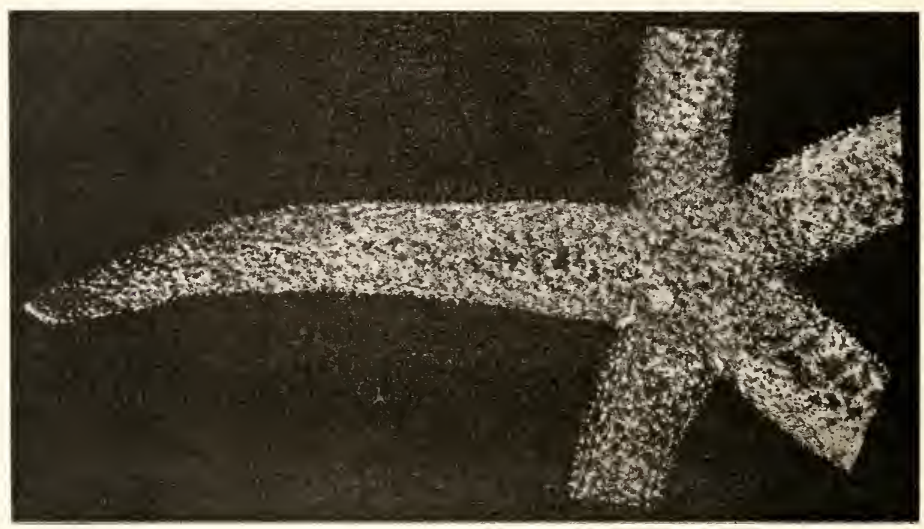

Fu. 22. - Echinaster sepositus; face dorsale ; $X \geq 3$.

L $E$. sepositus est très fréquent sur nos cotes de Provence, où il se montre à une faible prof. sur le pour tour des prairies de zostères associé à des Ilolothuries littorales et au Paracentrotus lividus, ou parmi les Algues, mais il peut descendre à de plus graudes prof. et on le rencontre assez sourent dans lit " broundo ", vers 40 m. où sa taille est nime ordin. plus grande que chez les indis. littoraux. On le retroure dans un grand nombre de local. de la Méditerranée. 11 se montre égal. sur nos côtes de l'Atlantique à mer basse ou à de faibles prof.. et il s'étend sur les côtes de Bretagne jusquäa Roscoff. localité qu il ne parail pas dépasser vers le $\mathbf{X}$.; il descend sur les cotes d'Afrique et on le trouve i Maclère et aux iles du Cap Vert. Il a été dragué à une prof. de $250 \mathrm{~m}$. Perrier le cite à $1.060 \mathrm{~m}$. (Bonifacio),

\section{G. HENRICIA Gray Cribrella Forbes.}

La face dors. "st formée le pl. disposies an un reseau compact et chacune l'elles supporte de nombreux petits piq. serrés, non articulés sur un mamelon distinct. Sur les côtés des bras qui sont arrondis, les pl. tendent à se disposer en rangées longit., et l'on distingue une rang. marg. dors, et une marg. ventr. Sur la face ventr, les pl. forment ordin. de petites rangées transv. Des papules nombreuses mais isolées se montrent sur la face ventr. comme sur la face dors. Les sillons ambul, sont très élroits: les tubes forment 2 rangées et portent une rent. 
term.; le piq. adambul. int., |qu'il est difficile d'apereevoir entre les tubes ambul., est un peu comprimé et recourbé en lame de sabre. Pas de pédic.

Le g. Henricia se distingue du g. Echinaster par ses piq. non articulés et par la présenee de papules sur les deux faces du eorps.

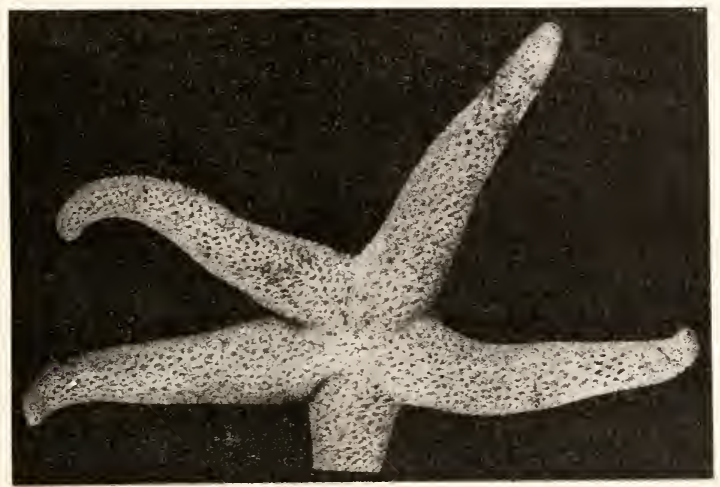

Fir. 23. Heniciu sanguinolenta; face dorsale; gr. nat,

H. sanguinolenta O. F. MüLler Cribrella s. Lütkex, Cr. oculata Pexiant]. Fig. 23. - Voir : Dexcan et Sladen, 1881, p. 31, pl. II, fig. 18-21 : Bell, 1892, p. 95.

Liespèce est de taille moyenne et sur nos coles elle reste plutôt petite : les bras ont 40 à $50 \mathrm{~mm}$. de long. en movenne, mais ils peuvent atteindre $70 \mathrm{~mm}$.; ils diminuent progress. de larg. jürqu'à l'extrémité qui forme une pointe arrondie; le disque est assez petit. Les piq. adambul. sont au nombre de 4 à 5 , disposés en une rangée transv, un peu irrégul.

La couleur à l'état vivant est d'un rouge assez foncé, et même rouge pourpre; sur les grands exempl., elle est beaucoup plus claire en dessous ; les petits indiv. sont aussi plus clairs; ces couleurs disparaissent complèt. dans l'alcool.

L'H. sanguinolenta est essentiellement littorale; elle est commune sur nos côtes de l'Atlantique et de la Manche, mais fait complèt. défaut en Méditerranée. On la trouve à mer basse sous les rochers, mais elle peut descendre à une assez grande prof., et a même été signalée à $2.400 \mathrm{~m}$. Elle remonte très haut dans les mers du X., jusqu à $81^{\circ}$ latit. $\mathrm{N}$.. et elle descend jusqu'aux Açores. Elle existe égal, sur les côtes des États-Lnis. 


\section{F. ASTERINIDÆ (ik.).}

Le squeleftr est formé de pl. imbriquées portant des pief. très courts réunis en petits groupes: parfois des groupes de deux constituent des rudiments de pédic.; les placques interrad. ventr. sont disposées en rangées longit. et transv. et portent de petits pirf.; les bords du corps sont tris minces. Le corpes est plus ou moins exact. pentagonal,

\section{G. ASTERINA I.IRDO.}

Le corps est pentagonal arec les cotés plus ou moins excarés et les angles arrondis; la face dors. est constituce par des pl. imbriquées portant des groupes de petits piq. très courts et assez serrés, entre lesquels se montrent des papules isolies, mais qui font défaut dans la partie ext. des aires interrad. ainsi que sur la face reutr. Les pl. portent des groupes de pir. moins nombreux, moins serrés et plus forts que sur la face dors. Les bords du eorps sont amincis et limités par 2 rangées de pl. marg., très petites mais bien distinctes. Les tubes ambul., bisériés, sont trrminés par une rent.

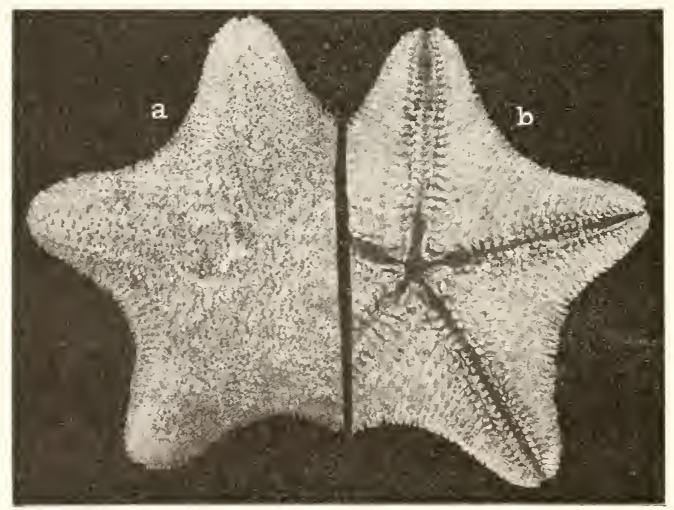

Fig. 24. - Asterina gibbosa; a, linec dorsale: b, face ventrale: légèrement grossi.

A. gibbosa Pixint Asteriscus verruculatus Wiun el Trosches.] Fig. 24. - Voir : l.xpwig, 1897, p. 207, pl. V. fig. j̀ à 8 .

Les cotés sont assez fortement excavés, el daus certains échant. on peut dire qu'il existe des bras à la vérilé trè- courts et triangulaires, tandis que 
daus d'autres ces bras sont à peine indiqués; le rapport du $K / r$ varie de 1,2 a 1, \% Le diam. est compris habil. entre 35 et 45 mm, mais il peut atteindre fol et mème 67 mm.

Sur les indiv. non dénuclés, la face dors. est converte de piq. très courts, réunis par petits groupes de $\mathbf{t}$ a $\delta$, qui oflrent un arrangentent régul. en rangées transr. dans les aires interrad. On remarque souvent des groupes isolés de 2 piq. légèr. obl. ou mêne incurvés et formant ensemble un petit pédic. La pl. madrép. est rapprochée du centre du disque. Les papules sont assez nombreuses el elles se montrent sur me hande assez large daus les rég. rad, ainsi que sur la partie proxim. des régen. interrad. la lace ventr., complèt. dépourvue de papules, ofrre des piq. plus forts et plus allongés que les dors. : ils sont réunis par groupes de 2 on 3 disposés en quinconce, formant des rangées longil. et transv. Les piq. des pl. marg. ventr. sont ident. aux voisins. Les pl. adambul. portent chacune sur leur bord int. un petit peigne de 4 piq. dressés, il existe en plus 2 autres piq. s'insérant sur leur face ventr.

La coloration générale est verte ou vert jaunâtre, parfois un peu rougeâtre ou encore vert brunâtre, plus claire sur la face ventr., et elle est assez variable; elle disparaît complèt. dans l'alcool.

L'A. gibbosa est très répandue sur toutes nos côtes, aussi bien dans l'Atlantique qu'en Méditerranée. Dans l'Atlantique, on la reneontre à mer basse sur les rochers, contre les pierres; en Méditerranée, elle vit à une prof, de quelques dm., dans les Algues, contre les jetées des ports, les rochers, ete. Elle s'étend au N. jusqu'aux côtes d'Écosse et an S. elle atteint les còtes du Maroc, les Canaries et les Açores. Elle descend fréquenment jusquà $30 \mathrm{~m}$. de prof. et même elle a cité trouvée a Naples a $126 \mathrm{~m}$.

\section{(i. ANSEROPODA Namo Palmipes L. Agatsiz el anct, num. .}

Le gr. Anseropoda ayant été crés par Nando en 183', et le genre Palmipes par Agassiz en 1836, le premier terme a la priorité.

Le corps est extrêm. aplati et il rappelle par sa forme et par sat consistance une feuille de carton a hords tranchants; il est pentagonal, avec des cótés plus ou moins excarés et des bras courts et très élargis à la base. Il est courert de pl. très petites, disposées en rangées longit. et transs. rég., munies de très fins piq. Les papules sont localisées a une bande étroite qui s'étend sur la face dors. le long des rad. Les tubes ambul., bisérieis, sont ternines par une vent.

A. membranacea (Lixck Palmipes m. L. Igassiz. I). placenta Penvant)]. Fig. 25. - Voir : Bell, 189:, 1. 84. $\{$ P. placenta $\rfloor$, Ledwig, 1897, p. 343, pl. I. lig. 3 et 4 . [I'. membranaceus].

Le diam, oscille général. autour de 15 ('n. et peut atteindre $20 \mathrm{~cm}$. Les cótés sont assez profond. excavés et l'on peut dire qu'il existe des bras très larges à la base, triangulaires et à peu près aussi longs que larges ; le rapport $R / r$ égale 1,5 à 1,6 . Les bords sont quelque peu sinueux.

La face dors, est couverte de pl. très petites, formant des rangées longit. 
et transs. Irès régul. séparées par de légers sillons, et portant chacune un groupe de 6 à 10 piq. très fins el tres enurts. Les pl. de la ligne carin. sont à peine plus grandes que les autres, mais de chaque crité de cette ligne se trouvent 2 rangée un peu irrézul. de pores asse gros qui sont surtout développés dams la rég. eentrale du corps ct satténuent ou disparaisent vers lat partic term. des bras. La pl. madrép. est trés petite el voisine du centre. Sur la face ventr., les pl., un peu phas graudes que sur la face dors., forment anssi des rangées longit. el transs, : elles portent chacune mu groupe de pretits piq. lins, acérés el vitrax, disposes en ares. Les pl. dimiment de laille i mesure quion se rapproche des bords. Ces bords euxmenes sont limités par mue double rangée de pl. marge a peine phus gratudes que les roisines. les pl, antambul. portent sur leur bord. int. une rangée longit. de a piq. réunis sur une partie de leur lons. par me menbrane, et, en dehors, une rangée obl. de i piq. plus petils.

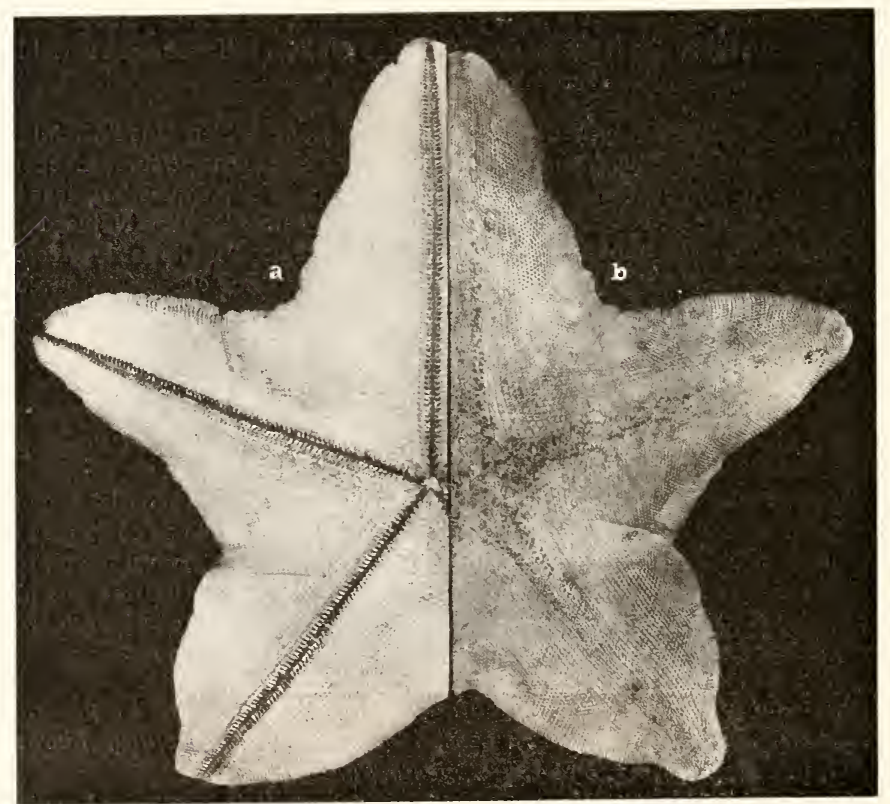

Fic. 25. - Anseropoda membranarea; a, faee ventrale; b, face dorsale; $X 1 / 3$.

Che\% le vivant, la face dors. es géméral d'un rouge écarlate, parfois rouge 
jaunâtre ou rosé; la face ventr. est rougeâtre, grisàlre ou jaunàtre et offre souvent une bande marg. de la mème couleur que la face dors., les tubes ambul. sont jaunes. Ces couleurs passent dans l'alcool.

1:A. membranacea est assez commune sur nos cótes de l'Atlantique et de la Mediterranée. Elle a été rencontrée daus de nombreuses local. de notre littoral, depuis La Rochelle jusquau Pas-de-Calais, dans des fonds vaseux et dans des graviers littoraux, i $10-20 \mathrm{~m}$. de prof.. lin Méditerranée, elle se trouve plutôt dans des fonds vaseux, de 30 i 80 m. Elle s'étend peu vers le $N$., mais elle existe cependant sur les côtes de Belgique et d'Angleterre. Elle peut deseendre jusqu'a $200 \mathrm{~m}$.

\section{F. SOLASTERIDÆ Perrier.}

Le suel. dors. est réticulé et certaines pl., plus grandes que les voisines, se soulevent en une tige saillante portant un laisceau de petits piq. (paxilles); les plaques adamlıul. portent un premier système de piq., parallèles au sillon, et sur leur face ventrale, un deuxième système perpendic, au sillon; les bras sont ordin. nombreus.

\section{(i. SOLASTER Fonbes.}

Les bras sont habit. nombreux et, dans l'espèce française, ils varient entre 10 et 14. Les pl. du squel, forment uu réseau assez serré; aux points de reneontre des trabécules, les pl. se surélèvent en une tige épaisse et courte, portant à son extrém. une touffe de petits piq., le tout formant une sorte de pax. Les bords des bras offrent une rangée marg. de pax, plus grandes que les autres. Les espaces membraneux du réseau cale. laissent passer des papules. Les tubes ambul., disposés sur 2 rangs, se terminent par une vent.

S. papposus (Lixck) [C'rossuster p. Mülder el Troschel] lig. 26. - Voir Bell, 1992, p. 89; Kowher, 1909, p. 111, pl. II, fig. fi et pl. IT, fig. i et is Crossaster p. .

Le nombre des bras varie : les échant. de nos cotes en ent habil. 1:2 a lí, tandis que dans les mers du I., e nombe fombe souvent i lo et meme is 8 ou 9. Le dinque est grand el les bras ont a pen pres la long. du layon du disque. le diam. fotal est de $15 \mathrm{~cm}$. en moyenne, mais il peut afteindre $20 \mathrm{~cm}$. Les bras ont la forme de triangles tres allongés, assez poinlus à l'extrém. La plaque madrép. unique el assez grosie, est rapprochée du centre. Le réseau calc, est assez liche ; les pax, dors. sont petiles et courles, les pax, marg. sont plus fortes el plus longues. Les piq. adambul. comprennent d'abord un peigne de $\mathbf{f}$ piq. allongés, subégaux, disposés un peu obliq. par rapport au sillon et, en dehor's, sur une saillie de leur face veutr., il existe un peigne transv, de 5 ou 6 piq. identiques aux précédents.

La couleur est très variable ef ello est géméral, assez vive, Somsont la face 
dors. tout entière est d'un pourpre foncé on d'un rouge jauntre avee parfois les bras plus clairs; ailleurs, les pédoncules des pax. ont une coloralion Pranchement verte on bien la face dors., qui est rouge, est lave de vert. La lace ventr. est plus claire et jaunalre. Ces colorations disparaisent dans l'alcool.

Le S. papposus est assez eommum dans le Pasted dalais et sum nos coles de lat Manche et il est assez friquemment rejele it la cite par les lempetes. II vit habit.

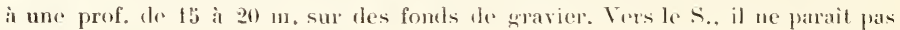
dépasser le $4 \tau^{\circ}$ latit. X. 11 est assez commun sul les coles didngleterte ef il remonte

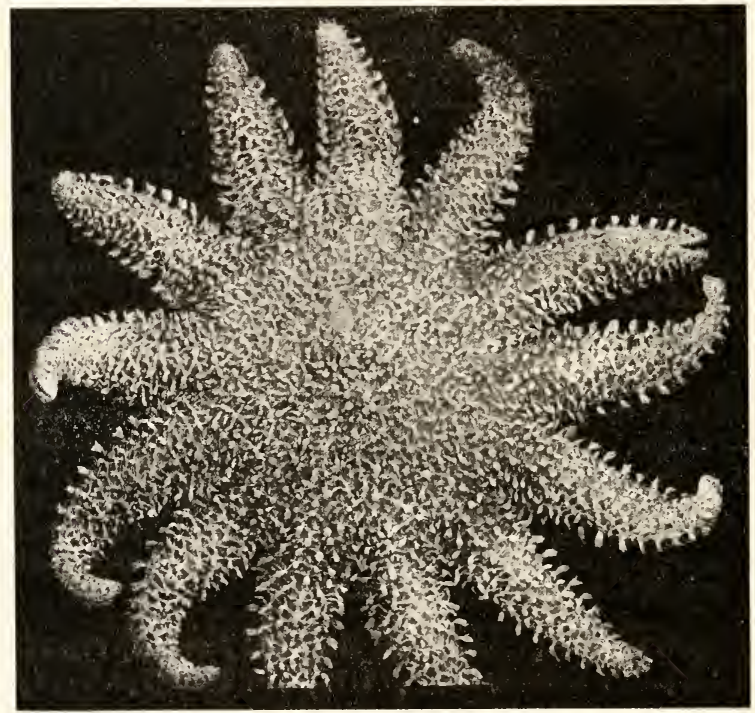

Viri. 26. - Solaster paprosus; fice dorsale; légère riduil.

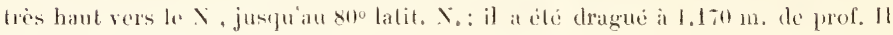
est inconnu en Méditerrance.

\section{CHATASTERIDÆ LUUWIG.}

Caractères du genre Chietaster.

G. GHÆTASTER M̈̈LLIR of TuOschel.

Les bras sonf très allongés, minces, cylindriques et le disque est très petit. Le

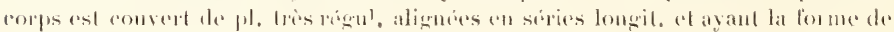


pax., e. ì d. quielles sont constituées par un peicloneule trìs court et épais portant sur sa face libre tronguée de nombrenx petils piq. serrés et vitreux. Sur les cotés des bras, on distingue 2 rangées marg. de pl, un peu plus grandes que les autres; enfin, entre les narg. ventr. et les adambul., se montrent quelpues rangées de pl. ventr. Il existe une pl, marg. impaire dans chanue sirie dors. ch ventr. Entre les

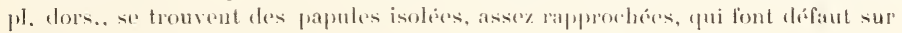

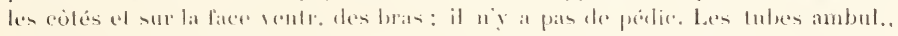
bisédićs, sont munis diuns venl.

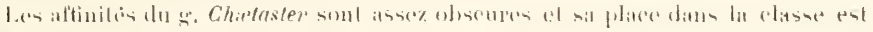
disentíce.

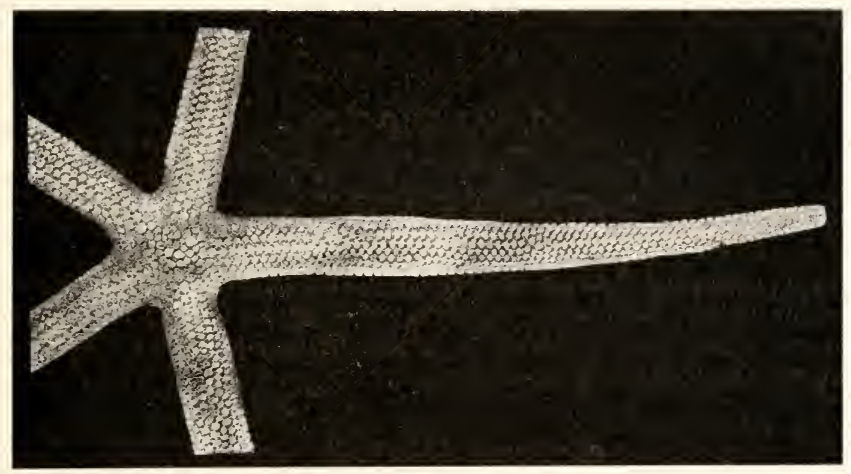

Fui. 27. Chietaster longipes; face dorsale; $X 2 / 3$.

Ch. longipes (Retzrs Fig. 27. - Voir : Lunwe, 1897, p, 131, pl. I. tig. 3 el í.

Le diam. Lotal varie entre 15 à $2.5 \mathrm{~cm}$, ; les bras sent très grêles et étroits, et le disque est tres petit : le rapport $R$ r varie entre 10 et 12 dans les grands indiv. : ces bras sont cylindriques et ils vont en s'amincissant très lentement jusquà l'extrém. Les piq. des pax. sont très courts, sauf ceux des pax. ventr. qui sont plus allongés et 3 ou 4 fois plus longs que larges. Ces piq. présentent une structure particulière : leur rég. basilaire seule est constituée par du tissu rétieulé ordin., tandis que leur moitié ou les 2 tiers ext. sont formés par un tissu transparent, vitreux et compact offrant à sa surf. quelques stries longit. Les sillons aurbul. sont très étroits ; les pl. adambul., éfroites égal. portent chacune jo piq., courts, mais qui ne sont pas terminés par une partie vitreuse, el offrent la structure ordinaire.

La face dors. est d'un jaune orangé, orange, ou jaune rougeâtre, ou encore d'un jaune de soufre; la face ventr. est plus claire. Cette couleur passe dans l'alcool. 
Le $C h$. longipes est rare. On l'a trouvé en différentes local. de la Méditerranée, La Ciotat, Nice, Alger, Naples, Palerme, Lesina, ete., entre 30 et $100 \mathrm{~m}$. de prof. En dehors de la Méditerranée, on le connaît sur les côtes du Maroc, au cap Palmas, aux Açores et aux Bermudes. Le Ch. longipes existe dans le golfe de Gascogne, et il peut remonter jusqu'au $45^{\circ}$ latit. N., où il a été trouvé par la "Princesse Alice " à $130 \mathrm{~m}$., associé à des Porania pulvillus, Stichastrella rosea, et Ophiothrix Lütkeni. On le rencontrera vraisemblablement en d'autres local. de notre plateau continental.

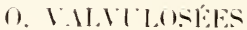

\section{F. OPHIDIASTERIDÆ VERRILL.}

L.es pl., imbriquées, forment plusieurs rangées longit. régul. entre lesquelles se trouvent des aires papulaires formant égal. des rangées longit.; les pl. et les aires sont uniformément couvertes de granules fins et rapprochés: le disque est très petit et les bras sont grands et allongés.

\section{G. "OPHIDIASTER L. Igassiz.}

Le disque est très petit, les bras sont plutôt gros, allongés, cylindriques ot ils conservent ì peu près la même larğ. jusqu au voisinage de l'extrém. qui est arrondie. Le corps porte des pl. aplaties, recouvertes, ainsi que les aires porifères intercalaires, par un tég. nuni de granules fins et serrés qui en obscurcit les contours; ces pl. sont disposées en rangées longit. Les aires porifères sont grandes, souvent même plus graudes que les pl. voisines, percées de nombreux orif. par où passent les papules et couvertes de granules identiques it ceux des pl. Les sillous ambul. sont assez étroits; les tubes ambul., bisériés, sont munis de vent. Les piq. adambul. sont disposés sur 2 rangées : l'int., formée de petits piq. cylindriques ef dresses, et l'ext. de gros piq. larges et aplatis, moins nombreux que les précédents et souvent couchés sur la face ventr.

*0. ophidianus (LAм.яск). Fig. 28. - Voir: Lunw1G, 1897, p. 300, pl. III, fig. 3 et 4 .

Le diam. varie entre 18 et $20 \mathrm{~cm}$. et peut atteindre $25 \mathrm{~cm}$. ; le rapport $R / r$ varie de 8 à 10. Les bras sont cylindriques, assez souvent rétrécis à leur insertion sur le disque et leur extrém. est arrondie. Les pl. dors. sont triangulaires, un peu plus larges que longues, avec le sommet prox.; il existe une rangée carin., une lat.-dors.. 2 mar... et enfin, sur la face ventr., 2 lat.-ventr. les aires porifères, at nomb. de 8 par bras, sont très grandes, arrondies, $u n$ 
peu plus grandes que les pl. voisines, surtoul celles de la rangée ventr. qui sont èlargies transteps. les piq. adambul. de la rangée int. soul alteruat. plus grands el plus petils; les plus pelits sont refonlés en dedans, Landis que les plus grands restent en dehors: les piy. ext., grpos el charpis, sont contigus dams le premier liess des bras. puis ils se separent par un intervalle al pen près égal à leur laren.

la couleur a l'état vivant est d’un rouge cammin lo's vif, parfois trè foneé, ou ronge orangé, un peu plus elair sur la lace ventr, ; les lubes ambul, sont jaunes; ces colorations passent dans l'aleonl.

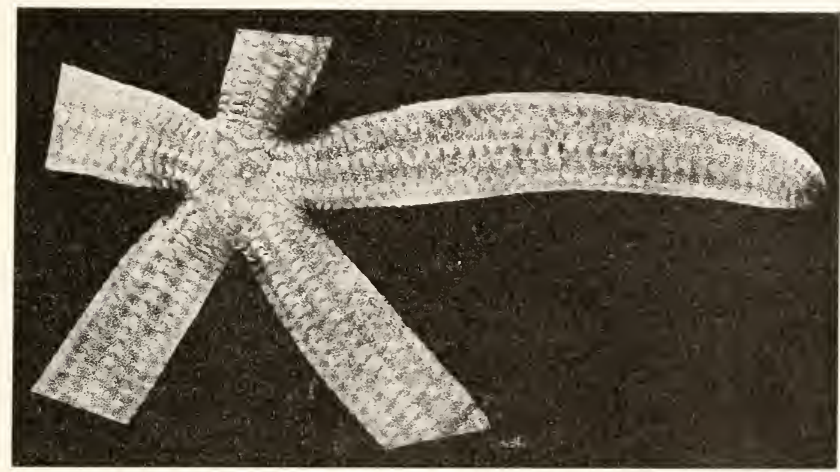

Fus. 28. - Ophediaster ophidianus; face dorsale ; $\times 1 / 2$.

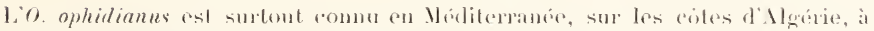

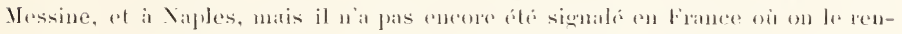

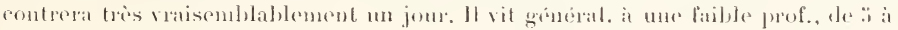
30 m., mais il peut descentre jusqu it $100 \mathrm{~m}$. En debors de lat Vediterame, on te comait aux Canares, aux Icores, aux iles du Cap Vert et it san-Thomé. If me parait pas penétrer dans les régions froides.

\section{G. HAGELIA Gray.}

Le distue est un peu plus grant que daus l'espece precédente; les bras, dargis a la base, rout en se rétrécissant assez rapidement justjüi l'extrém. qui est pointue, et enfin la face rentr. offre, entre les pl. marg. rentr. et les adambul., 3 rangées distinctes de pl. lat.-ventr. an lieu de 2 comme chez l'ophidiaster ophidianus. Les aires porifères forment 2 rangées principales, au lien d'une seule, entre les nrarg. ventr. el les adambul. ; et meme, à la base des bras, sur les grands échant., la rangée int. se dédouble en 2 autres dans chacune desfuelles les aires sont 2 fois plus petiles et 2 fois plus nombreuses que dans la rangée 
voisine. Les autres aires porifères et les pl. du corps sont disposées comme cher. l'Ophidiaster ophidianus. On reneontre assez souvent, mais non eonstamment, entre les granules de petits pédie, valvulaires.

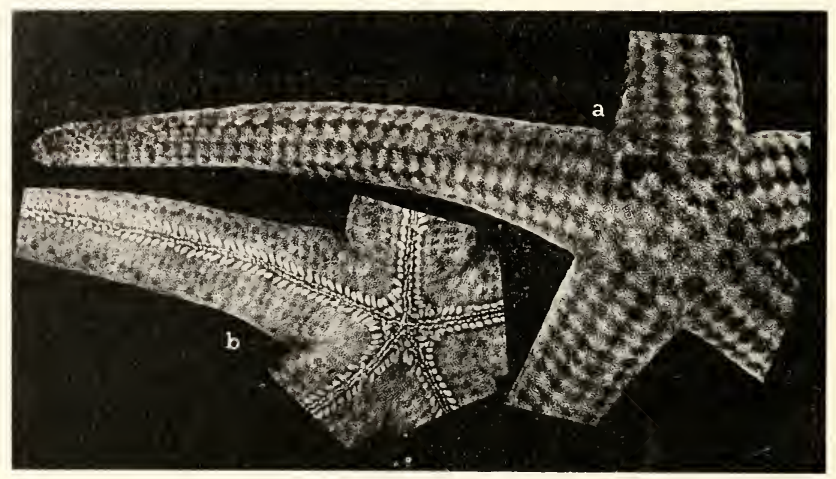

Fig. 29. - Hacelia altenuata; a, lace dorsale; b, face ventrale ; $X 2 / 3$.

H. attenuata (ins, Fig. 29. - Voir : Lrowig, 1897, p. 272, pl. III, fig. ti et 7 .

Le corps est habit. plus pelit que che\% l'O. ophidianus, mais il peut atteindre néanmoins $20 \mathrm{~cm}$. de diam. Le rapport $R r$ égale 5 à 6 . Indépendamment de la forme ext., on distinguera l/l. attenuata de l'espèce précédente par la présence, sur la lace ventr. des 3 rangées d'aires porifères que je viens d'indiquer.

La couleur à l'état vivant est d'un rouge écarlate che\% les grands indiv., et d'un rouge jaunâlre chez les petits; la face ventr. est plus claire; les tub. ambul. sont jaunitres; ces colorations disparaisisent dans l'alcool.

L'H. attenuata est rare; clle existe en difficents points de nos cotes méditerranéennes, notamment ì Xice, à La Ciotal, où je l’ai dragućc à une prof. de :30 m., a la limite des fonds coralligènes et des sables vaseux. Elle a été indiquée à Naples, sur les côtes de Sicile, et elle prut descendre jusquä $150 \mathrm{~m}$. Eu dehor's de la Méditerranée, on ne la connait quaaux $\Lambda$ çores.

\section{F. ASTEROPIDÆ l'isHER.}

Le corps est couvert d'une membrane fipaisse cachant les pl. sous-jacentes la face dors, est fortement convexe of le corps est assez épais mais les bords sont 
amincis ol tranchants; les play. ventr. sont grandes et disposces en rangres trausv, ; les piq. sont géuciral, localisés sur les bords du corps.

\section{G. PORANIA Grav.}

Le corps est assez ćpais, trapu, pentigonal avee des cotés assez fortement

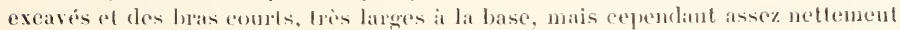
indiqués. La face dors. est converte d'un tég. épais, cachant complet, les pl.

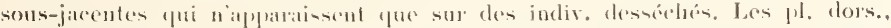

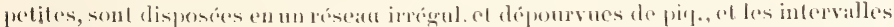

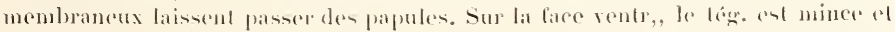
transparont at lon pent reconnater des pl, grandes, contignës, disposés en

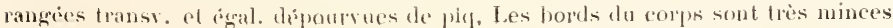

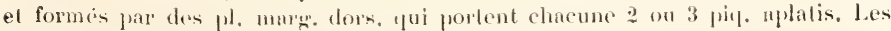

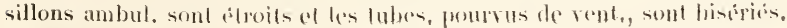

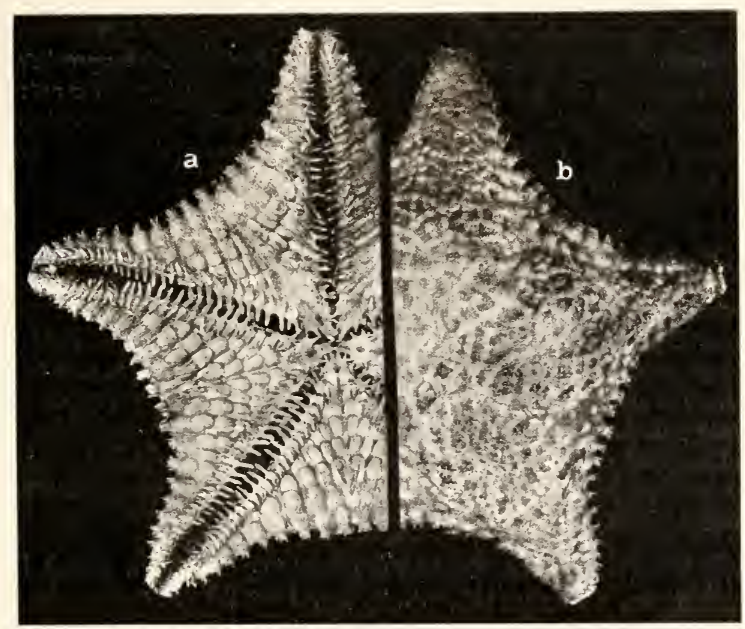

Fiti. 30. - Porania pulcillus, sehantillon desséché; a, face ventrate; b. fied dorsale: ligèrement réduit.

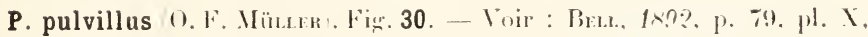
fir. 7 el 8 .

Le dian. varie ordin. entre sel $10 \mathrm{~cm}$. mais il pent alleinderexerptionn. 13 a lí com. Le dieque en large ; les bras sonl triangulares, eourts, a peu près ausi larges a la hase que longs ; le rapport $R /$ r égale 2 en moyenne. Le corps est asse\% epais avec les bords très amincis. Chez J'animal vivant, on 
ne distingue habit. sur la face dors. qu'un lég. épais el mou, parsemé de groupes de pilpules. La pl. madrép., prande, es placée ì égale distance du centre el des bords. Les pl. adimbul. portent chacume ㄹ piq.. les ext. beaucoup plus dével. que les inl.

A l'élal vivant, la couleur est d’un rouge général. Irès vif, écarlate ou pourpre sur la face dors. : la face ventr. es plus claire; celte coloration disparait dans l'alcool.

La P. pulvillus est incomme en Víditerranée. Sur nos eotes de l'Atlantique. elle se reneontre assez fréquemment sur le plateau continental : on peut la traguer ì partir d'une profondeur de $20 \mathrm{~m}$., mais elle est plus commune vers $100 \mathrm{~m}$. et descend jusqu à 200. Ellı est eonmue sur les côtes d'Ingleterre et remonte jusqu'à celles de Norvige.

\section{F. GONIASTERIDÆ FoR BH.S.}

Le squelette dors. est formé de pl. grantes, dpaisses et rapprochées, polygonales et eourertes de gros granules serrés, intre lesquels se montrent des papules isolées ; il existe sur tout le pourtour du corps une bordure très tistinete de grandes pl. marg. dorsales et ventr., igalement poursues de granules: les tubes ambul, sont terminés par une vent.

\section{G. CERAMASTER V'trmil..}

Le corps est pentagonal avee les faces dors. ot ventr. planes et parallèles ; il est couvert de pl. grandes, régulier. dispostés en rangrás longit. et obl. et eouvertes do gramules. Sur la face dors., ces pl. sont "labuleses ", ce a d. quelles ont la forme linn prisme tres surbaisse dont la surf. libre porte les gramules. Les pl. marg. ders. ot ventr. sont grandes, peu nomberuses, courertes de gratuules qui penvent manpuer dans lem regr. centr. les sillens ambul, sont étroits : les tubes sont bisérios et lermines frar une vent. Les plo adambul. portent de.

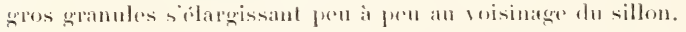

C. placenta Mïmas el Tuascmal Pentagonaster pl, ancl. Fig. 31. - Voir : Lamwa, 1897. p. 157, pl. V, lig. 1 al 2 Pentagonaster.

Le corps est presque exactement pentagonal avec lescótés légèr. incurvés; le rapport $R r=1,2$. Le diam. est ortin. de $10 \mathrm{~cm}$. en morenne, mais il peul arriver it lis am. Les angles du pentagone tantiol se terminent en une pointe asse\% marquée, liublit restent obtus. Le conps est solide, résistant

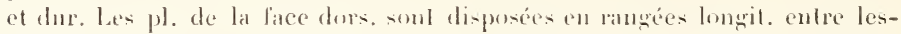
quelles restent de pelits ospaces par oi passont les papules. la pl. madrép., asser grande, es un pen plus rapproche du contre que des bords el elle se

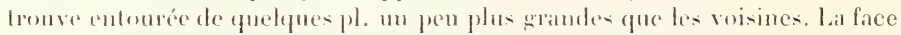
ventr. est converte de ple plus grandes que sur la lace dors., disposées en quinconce el mumies de gramules un pen plus gros. Le corps est limilé par de 
grosses pl. marg., les dor's. plus apparentes que les ventr., au nombre de 12 at 16 de chaque cóté. Parmi les granules de la face dors., on peut trouver ça et là quelques petits pédic. formés par உ2 valves minces et allongées. Les pl. adimbul. portent de gros granules disposés en plusienrs rangées : la rangée int. comprend jo gran. un peu allongés et la deuxième seulement 3: ensuite viemnent des gramules assez irrégul, disposés en 3 rangées plus ou moins apparentes; cen derniers granules ne sonl guere plus gros que ceux des pl, ventr. voir.

Lat couleur à l'étal vivanl est jaune brun ou brun rongeàtre ou encore rouge brique sur la face dors. : la firce ventr, est plus claire. Cies colorations disparaissent dans lialcool,

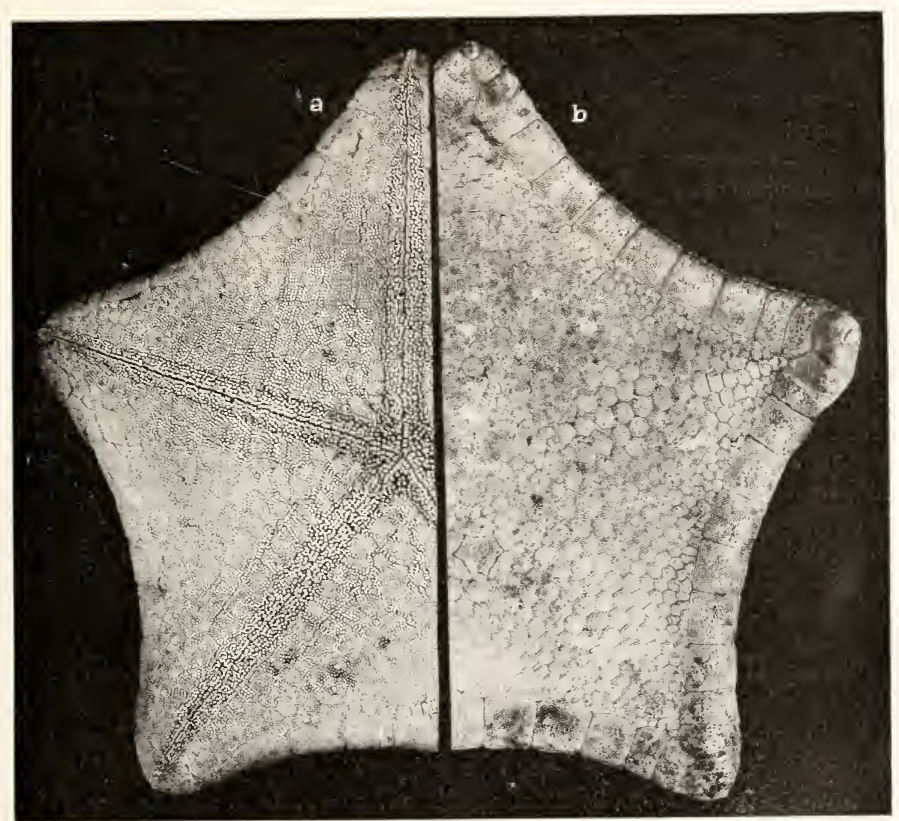

Fig. 31. - Ceramaster placenta; a, face rentrale; b, face dorsale; légèrement réduit.

Le G. placentu ast ansez rare: il a stá eousidéré pendint longtemps comnu. spécial à la Méditerranée at il était surtout connu dans le golfe de Ninjes ot dans l'Adriatique, entre 10 et $100 \mathrm{~m}$. el mème $160 \mathrm{~m}$. de prof. Je l'ai dragué dans le 
golfe de Gascogne a $400 \mathrm{~m}$. D'après Crivot, les clualutiers le péchent assez fréquemment au $\mathrm{N}$. W. du bassin d'Arcachon vers $190 \mathrm{~m}$., c. à. d, à la limite du plateau continental.

\section{(1) PAXHLOSLES}

\section{F. ASTROPEGTINIDÆ GRAY.}

Les pl. marg. dors. et ventr., très développées, forment une large bordure aux bras; le squel. dors. est lormé par des pax. Le corps esl aplati et les bras Bargis a la base se continuent progressicement avec do discpue: les papules sont simples : un anus.

\section{G. ASTROPECTEN LNAK.}

Le corps est aplati: le disque est relativ. grand et les loras sont allongés: lit face dors. est couverte de pax. Les pl. marg. ventr. sont tris grandes; les marg. dors, vues d'en haul, forment au corjes une bordure très distinete : elle's sont couvertes de granules parmi lespuels peurent s'élerer 1 ou 2 piq. pho ou moins développés; sur les bords lat. des pl., ees granules font place ì de fins piq. serrés, qui s'entre-croisent avec les piq. identiques des deux pl, adjacentes. Très fréquemment, la partie centr. du disque se soulève en un cône plus ou moins allongé, le cône aboral, qui persiste sur les ́chant. 'n alcol. Les pl. ventr. sont fort peu dével. ef les aires interrad. ventr. sont tris petites. Il n’existe pas de pédic. Les tubes anbul., bisériés, sont dépourrus de vent. Les papules sont simples.

A. aurantiacus. Fig. 32. - Voir Lcomig, 1897, 1) 3, pl. I1, fig. 1 el 2 .

Le diamètre alteint facilement 50 a 55 ( $\mathrm{cm}$. Le disque n'est pas très grand, et les bras ne sont pas trop élargis à la base; ils samincissent lentement et leur extrémité n'est pas pointue ; ils sont plus élroits que chez le Tethyaster. subinermis voir fig. 40 , el comme les pl. marw. dors. sont larges, l'aire occupée par les pax. reste assez étroile. La pl. martrép. est rapprochée des borls du disque. Les pl. marg. dors. sont couvertes de granules aplatis et portent tomjours chacme au moins un piq. conique, pointu, très appa-

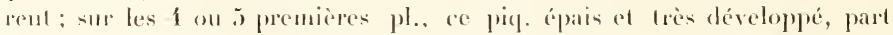
du bord int. de la pl., puis, au dela de la cinquieme, le piy. passe vers le milien de la pl., non pas progress., mais brusquenent el il se continue aimsi jusqu’à l’exfrém, des bras. Toutefois les quelques pl. qui suivent la 
cinquième continuent à oflrir sur leur bord int. un petit piq. de telle sorte que ees pl. ont a la fois 2 piq., un int. plus petit et un ext. plus grand ; ce piq. int. disparait vers la dixieme ou la douzieme. les pl. mirg. ventr. sont couvertes de graubles aplatis et portent sur leur bord distal ía jogros piq. aplatis el pointus; l'ext., presque, 2a fois plus long que les précédents, est très grand et très pointu : il est dirigé obliq. en dehors des bras el sil lomg. peut alteindre 12 ou $13 \mathrm{~mm}$. Les aires interrad, ventr. sont très petites et occupées par quelques pl. seulement. Chaque pl. atambul. porte d'abord sur sou bord int. 3 piq. forts et allongés, un peu aplatis, arrondis à l'extrém., le médian un peu plus grand que les autres, formant ensemble un petit faisceau dirigé obliq. vers le sillou, puis, sur leur lace ventr., il existe 2 autres piq. un peu plus petits, el enfin en dehors, un certain nombre de piq. encore plus pelits.

Chez l'animal vivant, la lace dors. ollre surtout une teinte orangée plus ou noins rouge: les pl. margin. dors. sont d’un jame orangé ; les pax. du milieu du disque, ansi que eelles de la ligne médiane des bras, el souvent aussi les pax. lat., oflrent une coloration jaunatre el font antant de petites taches arrondies sur le fond rouge orangé de la face dors. : la lace ventr. et les tubes ambul, sont jaunes ou d'un gris jaunatre très clair. Ces colorations passent complet. dans l’alcool.

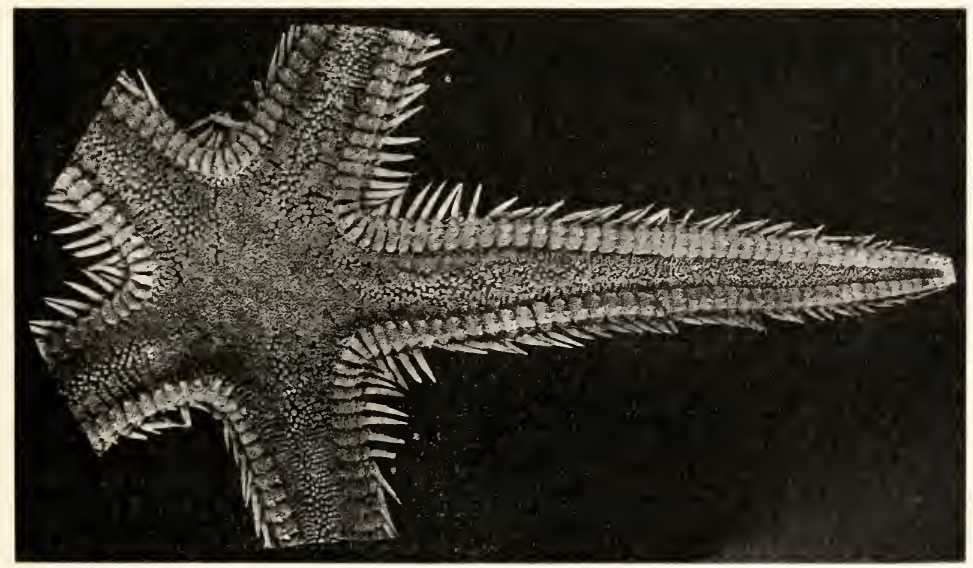

Fig. 32. - Astropecten aurantiacus; face dorsale; $\times 1 / \mathrm{i}$.

L'A. aurantiacus est très répandu en Méditerranée, de Banyuls à Nice, aux Baliares, sur les cotes d'Agérie et dans de nombreuses autres localités; sur les 
eôtes de Provenee, on le rencontre au pourtour des prairies de Zostères et en "broundo" de 5 i $50 \mathrm{~m}$., mais peut descendre jusquà $100 \mathrm{~m}$. Dans l"Atlantique, l'A. aurantiacus existe sur les eotes dı Porlugal, à Sietubal, et des cend jusquanx Canaries et à Madere, mais il semble disparaitre au S. de ees local. et etre remptacée par l'A. grureli que jai décrite on 1911.

Par sal laille, J.A. aurantiacus se distingue de tous les autres Astropecten de nos cotes ef ne pourrait itre confondu quave le Tethyaster subinermis qui atteint des dimensions anslogues: jündiquerai plus loin les ditférences qui séparent les deux esperees.

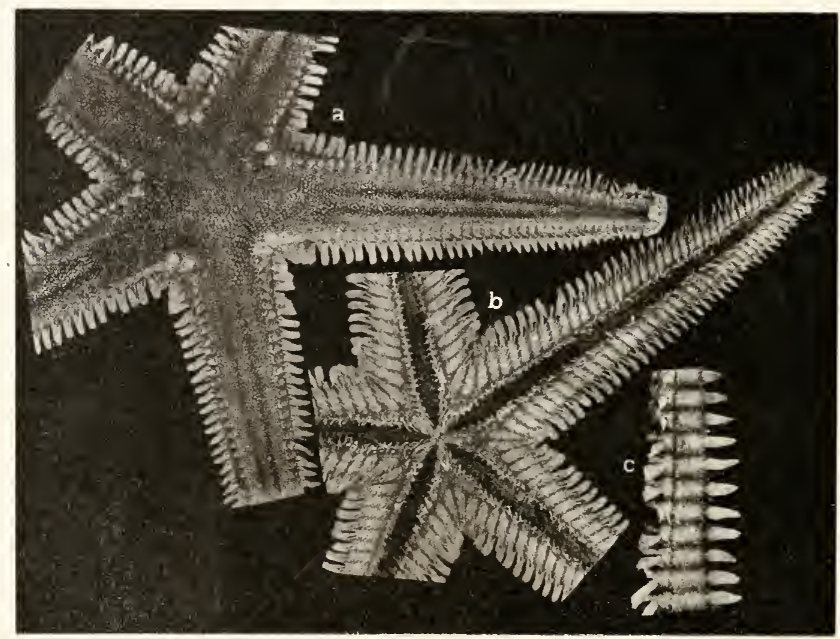

Fig. 33. - Astropecten bispinosus; a, facre dorsale: b. face rentrale; c, rue laterale d'un bras ; legirmenent réduit.

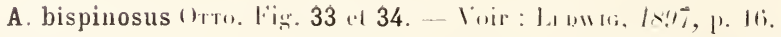

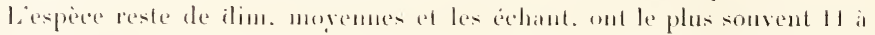

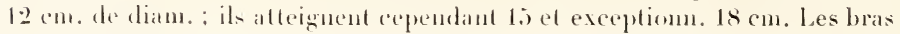

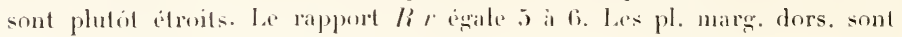
hautes. mais etroites, surtout dams lat premiere moitie des bras: leur face dors. est tris réduite tamdis que leur fice ext. verlic. est grande el quadrangulaire; dans la deuxieme moitie des bras, ces faces vertic. deviemment de plus en plus basses landis que les faces dors. súlargissent un peu. La face dors. de chaque pl. est presque toute entière oceupée par un gros piq. dressé

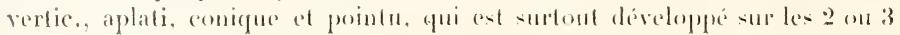


premières pl. de chaque rangée. Ce piq. est fout it fail caractéristique de l'espèce; les faces lat. vert. sont nues sur la plus grande partie de leur surf. et elles offrent seulement vers leurs bords quelques petits piq. Les pl. marg. ventr. sont aussi nues sur la plus grande parlie de leur surf. et ne portent de piq. que vers les borels : ces piq. sont plus petits sur le bord prox. que sur le bord dist. où ils sallomgent progress. a mesure quon s’approche du bord ext. de la pl., mais cest sultout le piq. exl. qui prend un très grand déveleppenent : sa taille dépasse mème celle du piq. correspondant des pl. marg. dors. Ces deux piq. lat. frappent l'wil quand on regarde l'Astérie par en haut el ils forment deux rangées très régul., d'où le nom de bispinosus donné à l'espèce. Les pl. adambul. portent chacune 3 piq. int. formant un petil faiscean dans lequel le piq. médian est le plus grand et se montre légér. recourbé en lame de sabre: la surf. ventr. des pl. porte une deuxième rangée de 3 piq. disposés obliq., le piq. prox. est notablement plus petit que les autres qui sont aplatis el lancéolés.

Sur le vivant, la face dors. est d'une couleur olivatre ou brunâtre plus ou moins foncée, les grands piq. marges. sont plus claiı's, jaune verdâtre ou blancs; la face ventr. est assez claire. Ces colorations persistent en partie dans l'alcool.

L'A. bispinosus est commun sur nos cotes de la Iéditerranée, depuis Banyuls jusqu à Nice où il vit à une très faible profoncleur, princip. sur le sable, en arant des prairies de Zostères, associé aux Zlolothuria tubulosa, II. polii, Echinaster seposilus, etc.; il descend jusqu“i 10 ou $130 \mathrm{~m}$. Il est très répandu dans la Méditerranée ainsi que dans l'Adriatique, sur les cotes d'Agérie et il peut descendre jusquäa $: 00 \mathrm{~m}$. En dehors de la Méditerranée, il n’a encore été signalé quaux Acores.

Lid. bispingsns se reeonnail imméliatement a ses 2 rang. de pị. marg. tris développés et il ne peut ètre confondu aree aneun autre Astropecten. Certains

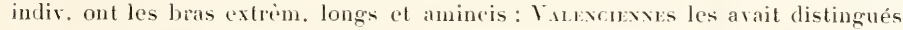
sous le nom d'A. myosurus mais ils ne constituent mème pas une variété distincte.

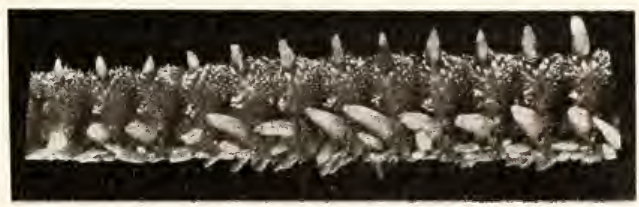

Fig. 34-Astropecten bispinosus, var. platyacunthus; vie latcrale diun bras; $X:$

VAR.platyacanthus (Philippi). Fig. 34. - Voir : Lenwig, 1897, p. 16, pl. II, fig. 6. Certains auteurs ont distingué autrefois comme espèce une forme qui est considérée maintenant comme une simple variété de l'A. bispinosus, et qui est caractérisée princip. par ses pl, marg, dors, el ventr. munies de petits piq. plus 
nombreux ef plus forts gue chez t.l. bispinosus lypipur. Les faces Jat, vertic des marg. dors., an lieu dietre nues en beur milieu, porlent l'assez nombreux petits

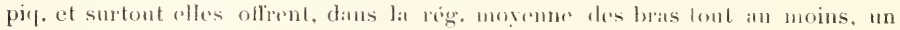
faiserau de ping plus grands que les voisins, dreseris of souvent convergents, an nomble de $i$ is 6 par pl. el formant une solte de prodic.

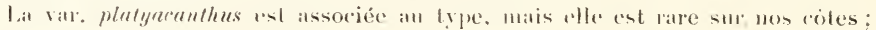

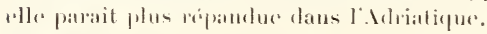

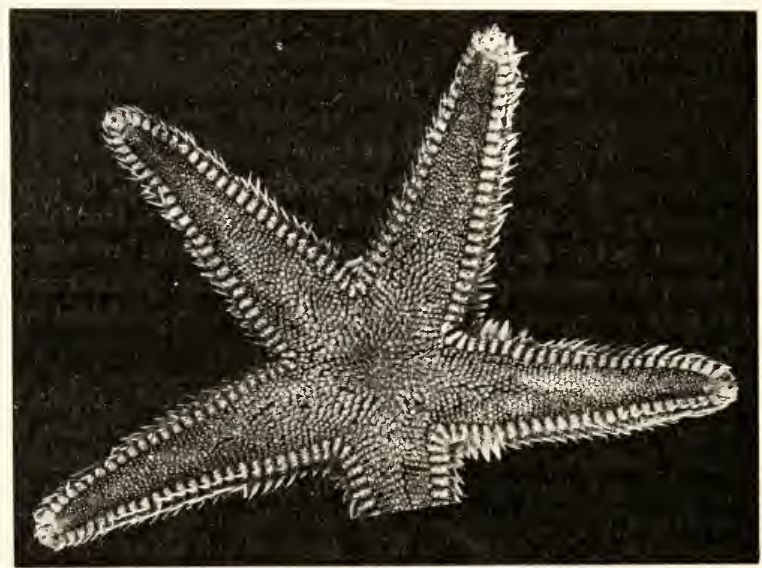

lig. 35. - Astroperten spinulosus : face dorsale : grandeur Halurelle.

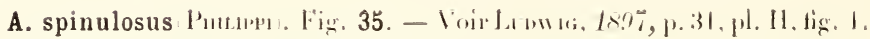
La taille reste lonjoms aste\% petite : le diam. est compris omlin. entre to on

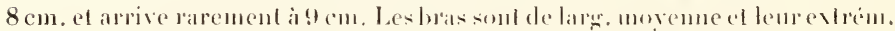

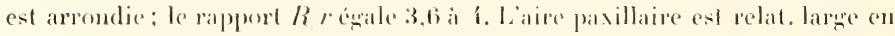

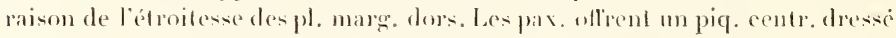

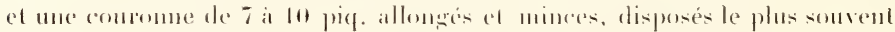

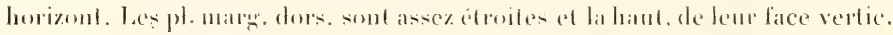

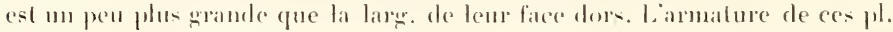
est asse\% variable : elle consiste ordin. ("n wranules un pen allongés, coniques el pointus, peu serrés, qui ne merilent pas le mom de piq., an milien desquels séleve mu rai petit piq. conique ef pointu qui se trouve d'abord rapproche du bord int. de la pl. sur les premieres pl. puis passe peu i peu sur le bord ext. Parfois, en dehors te ce piq. principal, il en existe nu deuxième plus petit et même un troisième. 1)ans certains exempl., les gramules sont plus allongés que dans d'autres: quelquefoi- enfin. la face exl. vert. des pl. offre 
un groupe de très petits piq., au nombre de 4 ou 5 , dressés et convergents, formant une sorte de petit pédic. : ćest en somme une variation analogue à celle que montre l'A. bispinosus var. platyucanthus. Les pl. marg. ventr. sont couvertes de nombreux petits piq. qui deviennent plus grands sur le bord distal ou l'on remarque surtout 3 grands piq. aplatis et pointus, l'ext. plus développé que les autres et débordant largement les bras. Les pl. adambul. présentent d'abord en dedans dı sillon un seul piq. int. légèr. incurvé et aplati ; ee piq. unique est nettement séparé des 2 piq. qui font suite et qui constituent la rangée moyenne: ces derniers sont aplatis longit, leur extrém. est tronquée et mime plus large que le reste du piq.; en dehors, enfin, viennent 2 autres piq. beancoup plus petits. Cette disposition des piq. adimbul. est tout à lait caractéristique de l'A. spinulosus.

La couleur de la face dors, du corps est brunâtre, brun-olivâtre ou vertrougeâtre, toujours assez foncée; les piq. lat. sont plus clairs ainsi que la face ventr. Ces couleurs sont général. conservées dans l'alcool.

Cette espèce n'a encore été trouvée qu'en Méditerranée; elle est assez répandue sur nos còtes el vit à une prof, très faible, 3 ou $4 \mathrm{~m}$. en moyenne, au milieu des Algues ou sur le sable. Elle est égal. commue à Naples, sur les eôtes de Sicile, dans l'Adriatique et elle peut descendre jusqu’à $50 \mathrm{~m}$.

A. jonstoni Delle Chinje [A squamatus Mülder et Troschel), A. aster Lütкex]. Fig. 36. - Voir Lidwig, 1897, p. 50, pl. II, fig. 3.

L'espèce reste toujours d'assez petite taille et son diam. oscille ordin. entre 5 et $6 \mathrm{~cm}$., il ne dépasse pas $7 \mathrm{~cm}$. Le disque est relat. grand; les bras élargis à leur base, sont triangulaires avee une pointe obtuse; le rapport $R_{r} r$ égale 3. La face dors. est couverte de pax. comprenant un cerele d'une douzaine de piq. périph. et un groupe centr. de 5 ou 6 piq., le tout offrant une disposition rézulière et álégante; les piq. sont finement spinuleux. La pl. madrép. est rapprochée du bord. Le's pl. marg. dors. ne sont pas trè trandes et leurs faces vertic. we sont pas très hautes; elles sont convertex de granuledesquels s'élève, à partir de la sixième, un petit piq très court, conique et obtus; ces piq. peuvent manquer plus ou moins complèt. dans certains exempl. Les pl, marg. ventr. sont grandes et surtout tres larges, $千$ fois plus larges que longues à la base des bras; presque toute leur surl. ventr. est nue et elles nolfrent à leur périph. qu'une simple bordure de squamules aplaties, qui, sur le bord ext. de la pl. sallongent en 3 ou '́ piq. aplatis et pointus, suivis d'un gros piq. ext. beaucoup plus développé, aplati égal., avec l'extrém. tronquée et qui déborde largement les bras. Les pl. adambul. portent d'abord un premier groupe de 3 piq. int. assez peu développés, le médian un peu plus fort, puis un deuxième groupe de 3 autres piq., le médian beaucoup plus long; enfin, en dehors, viennent quelques piq. beaucoup plus petits.

La face dors. offre une coloration générale verdâtre, gris-verdâtre ou brun verdàtre, ordin. assez claire; les pl. marg. dors. sont bleues ou grisbleu, et les piq. marg. jaune orangé avee, ì la base, une tache plus foncée; 
La face ventr. est d'un blanc jaunatre. Ces colorations disparaissent dans l'alcool.

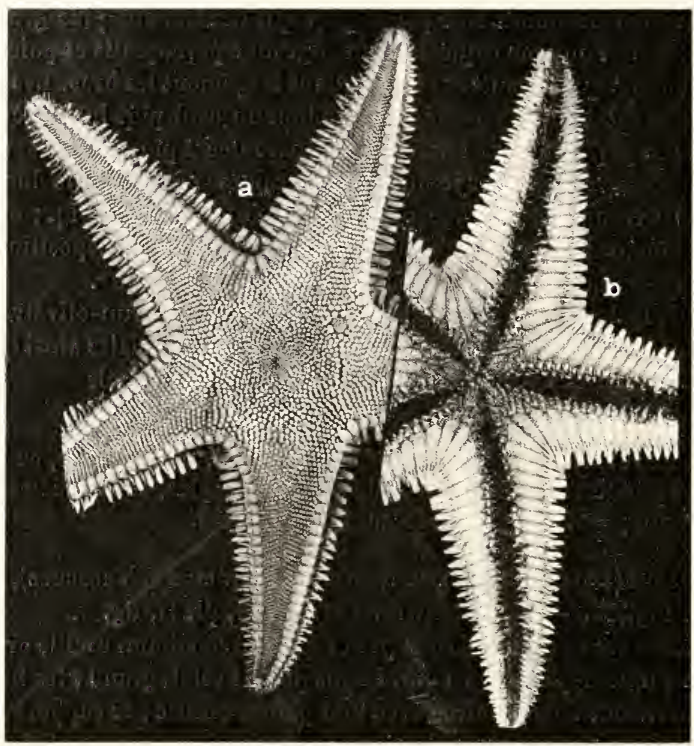

Fig. 36. - Astropecten jonstoni; a. face dorsale; b, face reutrale; légèrement grossi.

L'A. jonstoni existe seulement en Méditerranée. Sur nos cotes de Provence, on

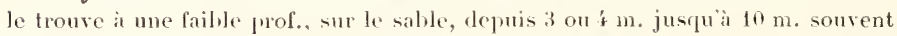
associé a l'A. bispinosus; il est connu ì Banyuls, it Marseille, ì La Ciotat, sur nos cotes dilgérie, a Xaples, it Messine, ete. loujours littoral, mais d'une manière générale il est assez rare.

A. irregularis Lixck, incl. A. pentacanthus Dele Chusf) el A. serratus Müı.ı el Truscm. . Fïr. 37, 38,39. - Voirprincipalement:

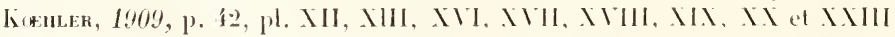
1. irregularis , Lamos, $1 \times 97$, p. 39, pl. II, lig. 5 A. pentacanthus.

Cetle espèce posside une très vaste extension wéspraphique. car elle s'étend depuis les cotes de Norvegze jusqu'a celles dusabara, el eviste égal. en Méditerranée, mais aussi elle présente d'assez grandes variations portant princip. sur l'armature des pl. marg. dors, de telle sorte que pendant longtemps les auteurs ont sepparé de l’.l. irregularis, en lant quespèces 
distinctes, l'A. serratus de l'Atlantique, dont les pl. marg. dors, portent plusieurs piq. très forts, et l'A. pentacanthus de la Méditerranée dont les pl. marg. dors. sont dépourvues de piq. J'ai montré en 1909 que ces 2 espèces ne devaient constituer que de simples variétés de l'A. irregularis et qu il y avait lieu de distinguer un $A$. irregularis que j’ai appelé typicus Fig. 37, un A. irregularis var. serratus (Fig. 39), et un $A$. irregularis var. pentacanthus Fig. 38 . Je ne puis donner ici que des indications très sommaires sur ces formes et je renvoie le lecteur à mou travail de 1909 .

Les dim. de l'A. irreguluris sont tres variables; les plus grands échant.

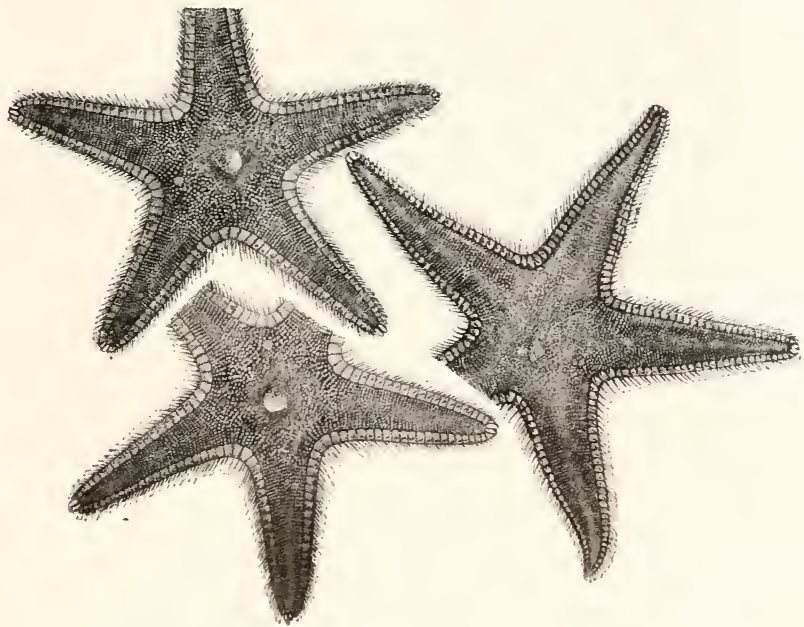

Fig. 37. - Astropecten irregularis typicus; face dorsate; $X 12$.

peuvent atteindre 15 ì $16 \mathrm{~cm}$, de diam., mais très souvent le diam. est comprientre 8 et $12 \mathrm{~cm}$. Le disque n'est pas trèr grand et les bras sont assez allongés: le rapport $R$, varie entre 3 , 5 et 5 . Laire paxillaire esl assez large ef la pl. madrép., grande, est rapprochée des bords. Les pl. marg. dors, sont, movennement développées: les pl. marg. ventr. sont courertes de squanules ou de petits piq. aplatis, tantot ovalaires, tantot un peu élargis à l'extrém.; leur bord distal porte plusieur piq. dont le nombre varie de 4 à 6 , et reste en général fixé ì 5 : ces piq. sont aplatis el pointus, et leurs dim. vont en augmentant jusquaux 2 piq. ext. qui ssnt grands, débordent largement les bras en dessous et sont visibles quand on regarde l'Astérie par en haut. Les piq. adambul. sont disposés sur 3 rangs et comprennent un premicr 
groupe int. de 3 piq. à peu près eylindriques, le médian un peu plus grand et légèr. recourbé, un deuxième groupe de 3 piq. plus forts que les précédents, aplatis avec l'exlrém. élargie et souvent tronquée, le médian plus grand, et entin 2 atutres piq. ext. plus petits.

L'armature des pl. marg. dors. est tres variable ainsi que je l'ai dit plus haut. En principe, chez l'A. irregularis de l'Allantique, chaque pl. marg. dors. est armée d'un piq. petit et conique, assez épais et rapproché du bord ext. de la pl. Dans certains exempl., ces piq. existent dès la première pl. marg, et ils sont mème plus développes sur les premières pl. : dans d'autres au contraire, ils manquent sur ces premieres pl. et n'apparaissent que vers la $5^{\mathrm{e}}$ ou la $f^{\mathrm{e}}$. Je piq. se distingne nettement des granules qui recouvrent uniformément la pl. Wans dautres échant. enfin, les piq. se réduisent à des

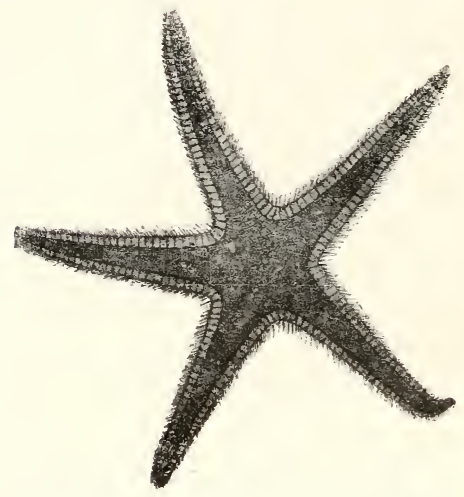

IN. 38. - Astropecten irregularis pentacanthus; lace dorsale: $X 12$. granules un peu plus gros que les voisins. En Méditerranée au contraire, les pl. marg. dors. sont, en principe, toujours dépourvues de piq. et c'est sur ce caractère quion a fondé l'espèce appelée A. pentacanthus. Cependant, en examinant un grand nombre d’indiv. méditerranéens, j’en ai rencontré quelques-uns, surtout parmi les jeunes, chez lesquelo les pl. marg. dors. portaient un petit piq. Ces indiv. armés de petits piq. sont absolument identiques i ceux de l'Atlantique. Réciproquement, on peut rencontrer sur nos cotes de l'Atlantique des échant. dont les pl. marg. dors. sont tout à fait inermes, associés à d'autres ayant les pl. marg. munies de piq. (Voir Honler, 190?, p. 46. En définitive, I'A. pentacanthus n'est que la var. inerme de l'A. irregularis.

Au contraire, les piq. marg. dors., anu lieu de se réduire et de disparaitre. peuvent, dans certains échant., prendre un grand développement et se montrer au nomb. de 3 et mème de $i$ sur la même pl., où ils forment alors une rangée transs. très apparente comparable à de petites dents de scie, d'ou le nom d'A. serratus. Cette forme a été rencontrée autrefois a La Rochelle et lanwig l'a indiquée en Méditerranée Messine). Ces indiv. à pl. marg. dors. très armées se relient par des intermédiaires aux A. irregularis typiques et ne peuvent étre considérés que comme une simple var. de ces derniers.

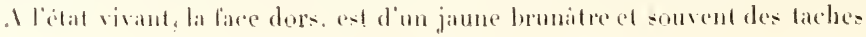


plus ton, ées dessinent une étoile sur le disque; la couleur est tantòt plus claire, tantôt pius foncée et elle peut passer au gris jaunâtre. La face ventr. est égal. toujours plus claire que la face dors. Ces colorations passent à peu près complèt. dans l'alcool.

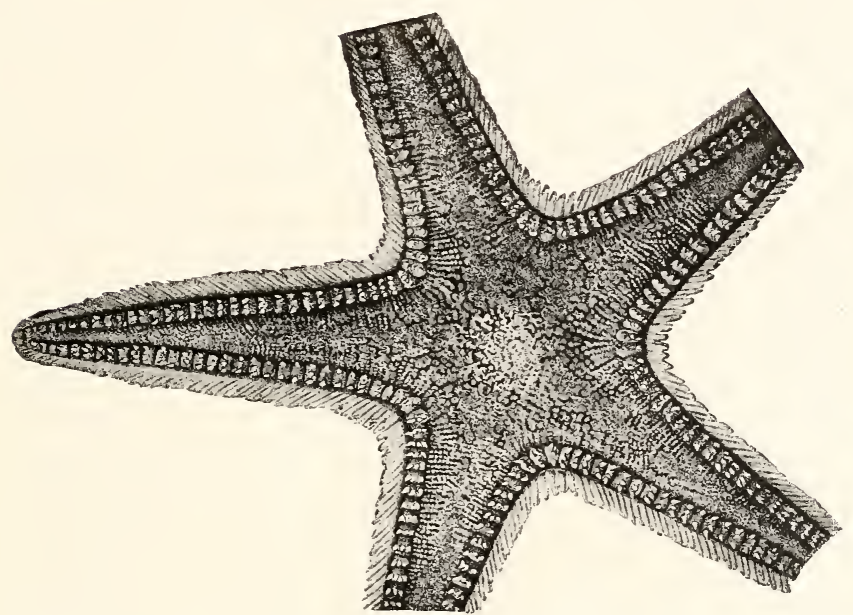

Fí. 39. - Astropecten irregularis serratus : face dorsale; légìrement réduit.

En Méditerranée, l'A. irregularis pentucanthus se trouve surtout dans les fonds vaseux à partir d'une dizaine de $\mathrm{m}$. de prof., mais il peut descendre beaucoup plus profond. et il a été signalé dans le canal d'Otrante à $929 \mathrm{~m}$. Dans l'Atlantique, l'A. irregularis est assez commun sur les plages et sur notre plateau continental, à des prof. variables depuis le niveau des basses mers jusqu'à 1.000 ou $1.500 \mathrm{~m}$.; il est très abondant sur certaines de nos plages sableuses, notamment à Arcachon, où il vit à quelques $\mathrm{cm}$. de prof., laissant dépasser sou cône aboral d'une long. de $5 \mathrm{~mm}$. environ. Il est aussi très répandu sur les còtes d'Angleterre.

\section{G. TETHYASTER SLADEN.}

Le corps est aplati ; le disque est relat. grand et les bras, allongés, sont élargis ¿ la base et ils vont en se rétrécissant régul. jusqu'ì l'extrém. qui est très pointue. La face dors. est couverte de pax. La pl. madrép., située à peu près à égale distance du centre et des bords, est bien apparente et elle n'est pas cachée par les pax. Les papules sont simples. Les pl. marg. dors. et rentr. sont grandes et très distinctes; les pl. dors, portent des granules qui, sur leurs bords 
adjacents, font place à de très fins piq. s'entrecroisant avee ceux de la pl roisine"; les pl. ventr. offrent quelques piq. peu développés, et les piq. ext. eux-mêmes sont peu visibles quand on regarde l'Astérie par en haut. Les aires interrad. rentr. sont grandes "t couvertes de pl. nombreuses formant plusieurs síries transr. Les pédic. font complèt. défaut. Les tubes ambul. sort disposés en 2 rangées et dépourvus de vent.

Le g. Tethyaster se distingue du g. Astropecten par les aires interrad. ventr. assez grandes, par les pl. marg, dors. courtes et larges et par les pl. marg. ventr. ne portant que de très courts pir.

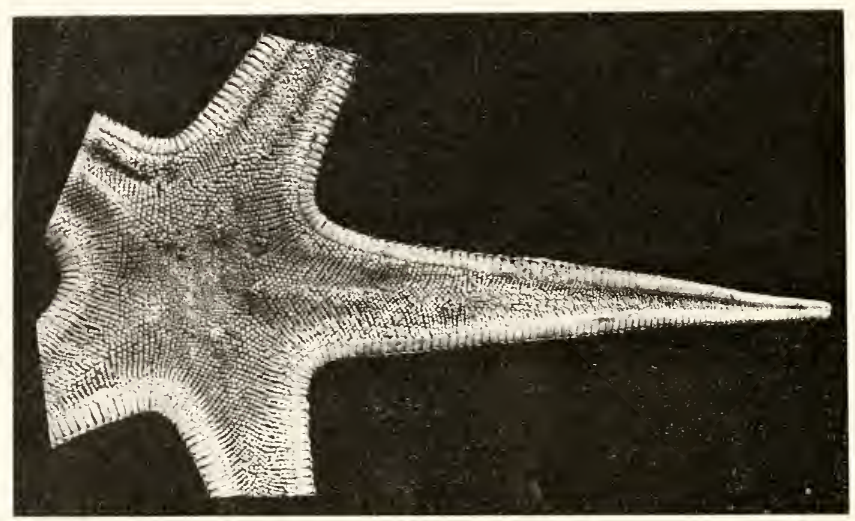

FIG. 40. Tethyaster subinermis; face dorsale; $X 1,4$.

T. subinermis Pinlippi Plutonasters. auct. T. Fig. 40. - Voir : Ledwig, 1897, p. 105, Pl. I. fig. 1 el 22 Plutonaster.

L'espèce est de l rès grande taille et le diam. peut dépasser $40 \mathrm{~cm}$. Le disque est tres qrand et les bras sont assez élargis à la base; le rapport $R$ r égale 3,6 a 3,9. La face dors. est couverte de jax. nombreuses et serrées, formant sur les bras des séries transv. obliques et l'aire qu'elles recourrent est assez large. La face dors. des pl. marg. dors. est un peu oblique : ces pl. sont ífois pluslargesque longues au moins au commencement des bras, et elles sont comvertes umiquement de gronules arrondis, sams la moindre indication de piq. Les pl. marg. ventr., un peu phus longues, portent des gran. aplatis, et, sur leur bord dist., 3 on 4 piq. aplatis, courts, coniques el pointus, appliqués contre la plaque; le plus ext. de ces piq. dépasse i peine les bords des bras. Les pl. ventr. forment des séries tres apparentes el sont couvertes de petits piq. Les pl. adambul. portent d'abord, sur leur bord int., un groupe de 3 piq.. grands, comprinces lat., obtus à l'extrém.. le médian un peu plus grand 
que les autres: puis, sur leur face ventr., plusieurs piq. plus petils, dont le nombre varie de 6 is 8 .

ua couleur a l'état vivant est rouge écarlate ou rouge orangé sur la face dors. et les pl. marg. sont d'un ton plus jaune: la face venlr. est jaume orangé ef les tubes ambut. sont plus foncés. Ces colorations disparaissent daus lialcool.

Le $T$. subinermis noest pas très rare en Méditerrance ef il at cté rencontré en diflérents points de nos eôtes, depuis Banyuls jusqüi Niee, entre 60 el $100 \mathrm{~m}$. de prof, ; il existe aussi a Naples, sur les cotes de Sicile, ete. En dehors de Ia Néditerranée, il peut deseendre sur les cotes d'Afrique jusquà Libéria. Il existe également dans le golfe de Gascogne oi je l'ai dragué entre 180 et $300 \mathrm{~m}$. de prof.

Par sa grande taille et sa coloration, le $T$. subinermis se rapproche de l'Astropecten aurantiacus, dont on le distingue de suite i labsence de prands piq. marg., a ses pl. marg. dor's. inermes, is son disfue plus grand, is ses aires interrat. ventr. bien diveloppées, ete.

\section{F. LUIDIIDE V'FrRH.}

Les bras sont étroits it lat bate, allongis, trie fragiles, et le distgue est petit; it n’y a pas de fl. margin. dor's., celles-ci étant remplacées par une simple rangée de pax.; les papules sont ramiliées ou lobées; pas d'amus.

\section{G. LUIDIA FuRBes.}

Le corps est aplati; les bras sont allongés el étroits ; la face dors. est couverte de pax. Les pl. marg. dors. sont indistinctes ear elles ne sont pas plus grosses que les autres pax. de la face dors. Les pl. ventr. sont fris peu nombreuses. Il existe des pédic. à 2 ou à 3 valves. Les papules sont divisées en plusieurs lobes. Les tubes ambul., bisériés, sont coniques et dépourvus de vent.

L. ciliaris Pullppl). Fig. 41, a. - Voir : Koenler, 1895, p. 3 et 1906, p. 50 ; Ledwig, 1897, p. 61, pl. IV, fig. 1.

Les bras sont au nombre de 7 , ils sont souvent incomplets et en voie de régénération : ce nombre de 7 est tout à fait constant contrairement ì ce qui arrive che\% d'autres espèces d'Astéries dont les bras sont en nombre sup. à 5 .

La L. ciliaris atteint une grande taille et le diam. peut mesurer $50 \mathrm{~cm}$. Le disque n'est pas très grand ; les bras sont assez minces, allongés el ils vont en se rétrécissant progress. jusqu'a l'extrém. qui est pointue. Le rapport $R$ 'r est éral à $x$ ou 9. La face dors. est couverte de pax, comprenant un gros piq. central ef 10 ou 12 piq. périph. plus petits. Ces pax. sont disposées assez irrégul., sauf vers les bords des bras où elles forment 3 rangées longit. bien régul. Les papules arec leurs lobes term. sont très apparentes suriout sur 
les côtés des bras. Les pl. marg. ventr. portent 4 piq. principaux : 2 int. dirigés obliqu. et 2 ext., beaucoup plus grands, s'étendant horizont. sur le bord des bras; ces piq. sont coniques et pointus. Les pl. adambul. portent chacune 2 piq. : l'int., légèr. recourbé, est un peu plus petit que l'ext. qui est droit. Il existe ¿ sortes de pédic., à 2 et 3 valves : les uns, qui se trouvent en dehors des piq. adambul., dans l’interralle qui séparé ceux-ei des piq. marg., ont 3 valves allongées et triangulaires; il existe général. un pédic. vis a vis de chaque pl, adambul. On reneontre, en outre, le plus souvent, un pédic. didactyle sur chaque pl. marg, ventr., entre le "' et le $3^{\circ}$ piq. Ces pédic. oflrent d'ailleurs certaines variations quant ì leur distribution : tantot ils se suivent régul. tantit ils manquent par place, et dans certains indiv. ils sont peu abondants : de plus les tridactyles sont parfois remplacés par des didaetyles dans la moitié dist. des bras.

La L. ciliaris est très délicate et fragile, et les bras se brisent aver la plus grande lacilité : le nom de L. fragillissima que lui avait donné Forbes élait très justilié. I l'état vivanl, la lace dors. est rouge ou rouge orangé et la face ventr. plus pâle; les tubes ambul. sont jaunes: ces colorations sont conservées en partie dans l'alcool.

La L. ciliaris est assez répantue au large de nos eôles. En Méditerranée, clle ent parfois rapportée par les pécheur's accrochée aux palangres qü̈ls calent sur nos eótes de Provence à des prof. de 50 à $80 \mathrm{~m}$; ; elle est connue dans le golfe de Naples. Elle se trouve égal. dans l'Atlantique, sur le plateau continental, à des prof, variant de $2 \mathrm{a}$ à $180 \mathrm{~m}$.

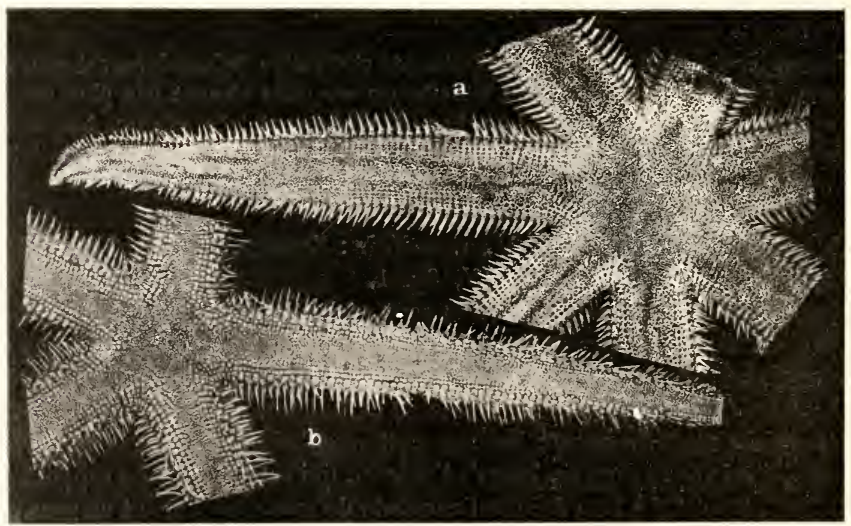

F16, 41. - a. Luidia ciliaris; face dorsale, $X \mathrm{t} / 3$; b. Luidia sarsi; litee dorsale, $X 23$. 
L. sarsi Dübex el Konex). Fig. 41, b. - Voir : Koenler, 1895, p, 6 et 1896, p. 51 ; Lidwig, 1897 , p. 85 , pl. IV. fig. 2.

I.a taille pent être assez grande et le diam, atteint $35 \mathrm{~cm}$. Le disque est plus petit et les bras sont un peu plus étroits que chez la L. ciliaris. Le rapport $R ! r=10$. Les pax. de la face dors. sont extrêm. serrées et constituées par des piq. plus fins que ehez la L. ciliaris; elles sont tout à fait confluentes, sauf sur les côtés des bras où l'on distingue 2 rangées longit. bien nettes. Les pl. marg. ventr. ne portent que 3 piq. principaux, le premier un peu plus petit que les 2 autres qui sont silués sur les côtés des bras. Dams les grands

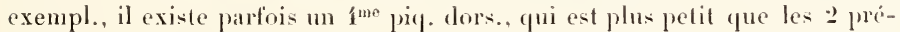
cédents, et qui est dressé obliqu. vers le haut. Les papules dors. offrent des lobes moins nombreux que chez la L. ciliaris, et elles sont localisées sur la partie centrale du disque et sur la région médiane des bras. Les pl. adambul. portent chacune 3 piq. aplatis : l'int. plus petit, en forme de lame de sabre, le médian plus grand et le dernier un peu moins fort. Les pédic. sont disposés i peu près comme che\% la L. ciliaris, du moins dans les grands indiv. : il y a en effel des pédic. entre les piq. adambul. el lespiq. mary.., el d’autres plus petits entre les of et $3^{e}$ piq. marg. mais ils nont jamais que 2 valves. biu général, les pédic. voisins des piq. adambul. sont aussi gros que rhez la $L$. ciliaris, il en existe parlois ’̨ el même 3 sur la mème ligne transversale.

La coulenr des échant, vivants est plus foncée que chez la L. ciliaris: elle est brunâtre, plus foncéc vers les bords des bras et sur les piq. marg. ; la face ventr. est plus claire et les tubes ambul. sont jaunâtres. Cette coloration passe plus ou moins complèt. dans l'alcool.

La L. sarsi vit surtout dans l'Allantique et se montre, sur notre plateau continental, a partir de :0 m. de prof. Elle remonte vers le $\mathrm{N}$. sur les cotes des lles Britanniques ef jusqüi celles de Norvige: d'autre part, elle a été reueontrie sur les cótes du Maroe à $32.2 \mathrm{~m}$. ; elle peut d'ailleurs descendre au-delat de $600 \mathrm{~m} .$. Ville existe également en Méditerranée (La Ciotat, Crìte).

Les 2 espèces de Luidia de nos cites aỵant constanment, l'une 7 bras et l'autre 3. se distinguent de suite l'une de l'autie. 


\section{OPHIURIDES}

Le corps des Ophiures est formé d'un disque arrondi duquel partent des bras minces el grèles général. lrès allongés; elles méritent, comme les Astéries, le nom d" "Étoiles de mer", mais elles se recommaissent de suite à ce fait que les bras sont tout a lait distincts du disque el quentre les bases des bras. les rég. interrad. du disque restent libres. Ces bras sont ordin. simples, quelquelois ramifiés fig. 43 ; ils sont en principe an nombre de 5 , rasement de fi on plus. L.̈̈ndépendance du disque et des hras est démontrée par la facilité aver laquelle on peut séparer le premier de coux-ci : cette opération qui peut etre réalisée chez toutes les Gphiures, s'effectue frès facilement dans les. Imphiuridées: on constate alors que les bras se continuent vers la bouche el qüils ne sont en aucune façon soudés avec les bords du disque comme chez les Istéries: le disque coille senlement leur rég. proxim. Bien mieux, il existe des espèces exotiques chez lesquelles cet arrachement, ectte amputation du disque parait ètre un phénomène normal qui permet sans doute me dissocialion des produits sexuels emportés avee le disque; celui-ci sera régénéré ensuite. Au point de vue anatomique, je rappellerai senlement que le disque renferme la tolalité du tube digestil ef les grlandes génit. el que les bras, formés d’art. suceessifs, au lieu d'être creux, comme chez les Istéries, sont occupés, dans chaque art. par 2 grosses pièces calcaires que l'on considere comme homologues aux pl ambul. des lstéries.

Le disque est général. couvert de pl. qui, tantot restent nues, Lantir portent des piq., des granules on des tubérosités. Jes pl. de la lace dors. du disque sont ordin. nombreuses, de petite taille, el parmi elles on distingue, vers l’insertion de chargue bras, une paire de pl. plus wrandes appelées les boucliers radiand ; souvent ansi on recomait, dans lit partie centr. du disque, une rosette de $f_{p}$ l. dites primaires, une centrale el 5 radiales, tantit contignès. tantot séparées : ces pl. ne représentent pas le squel. apieal prim. des lichoumdermes, mais elles résultent d'un arrangentent second. La lace ventr. du disque oflre en son eentre la bonche qui a me forme stellée. c i a d. qui offre jo prolongements rad. sétendant jusqu à la base des bras, 
et qui sont séparés par 5 rég. interrad. recouvertes de pl. spéciales dites pièces buccales fig. $45 \mathrm{c}: 60 \mathrm{~b}, 61 \mathrm{~b}$, ete.). Parmi celles-ci on distingue, en allant de dehors en dedans: une grosse pl. impaire, le bouclier buccal, qui, dans l'un des interrad., est percé d'un pore madrép. unique, puis $2 \mathrm{pl}$. paires, allongées, les pl. adorales, à la suite desquelles viennent 2 autres pl. paires plus petites, les pl.orales, et entin une série de petils piq. formant une pile vert.. les dents, qui s̈insèrent sur une petite tige vert. Les pl. adorales et orales portent ordin., sur leur bord libre, de très petits piq. appelés papilles buccales; en outre il peut exister, immédiatement en dessous des dents pr. dites, quelques autres papilles appelées dentaires.

Le squel. des bras fig. 42 comprend dans chaque art. 2 grosses pièces soudées chez l'adulte en une pièce unique appelée improprement la vertèbre (am), et qui occupe toute l'épaisseur des bras. Les vertèbres s'articulent les unes arec les autres par des saillies et des fossettes plus ou moins compliquées permettant des mourements. Ce squel. est complété par un squel. ext. comprenant i pl. par art. et qui sont disposées en rangées longit. rég.; il existe une rangée de $p l$. brachiales dorsules (ds), une de pl. brachiales

Fig. 42. - Coupe transversale schématique d'un bras d'Ophiure. ds, plarues brachiales dorsates; bs, plapues brachiales rentrales; ss, plactues brachiales latérales ; ac, piquants ; am. verlèbres; ra, tube aquifère radiaire; vte, canaux se rendant aux tubes ambulacraires te; rn, cordon nerveux radiaire; rv, cordon plastidogène radiaire (d’après $R$. Perrier).

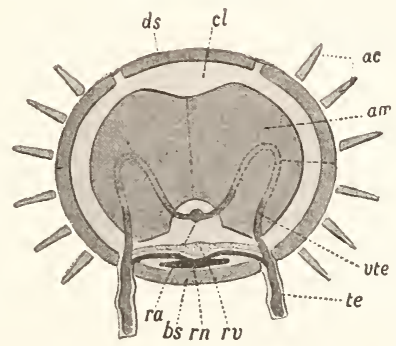

ventrales (bs et 2 rangées de $p l$. latérales (ss portant des piquants (ac Entre les pl. ventr. et les vertebres, se trouve un espace très étroit, prolongenent de la cavité générale, et rentermant le canal aquilère rad., le cordon nerveux, le cordon plastidogène ra, rn, rv, ele. Le canal aquilère ra. lournit i droite et à gauche des canalicules aboutissant aux tubes ambul. (te et passant entre lespl. brach. ventr. et les pl. brach. lat. par un orif. général. muni d'une ou plusieurs écailles appelé le pore tentaculaire. Il n'existe pas de vésic. ambul. et les tubes ne sont jamais terminés par une vent. ; its ue servent pas à la locomotion qui est réalisée simplement par le-mouvenents des bras et des piq. (1n admet que les pl. lat. sont homologues aux pl. adambut. des Astéries el que tes pièces buecales résultent de la transtormation de certaines pl. des 2 premiers art. brach.

Le tube dig. remplit presque complèt. la cavité du disque; c'est un simple sac stomacal. Lne partie de l'espace qui reste libre entre le sac et la paroi du corps est occupée, dans les rég. interrad, et de chaque coité de la base des 
bras, par les bourses génitales. Ce sont des poches s'ourrant chacune au dehors par une fente longit. étroite qu'on aperçoit sur la face ventr. du disque, de chaque coté de la base des bras fig. $44 \mathrm{~b}$ : le long du bord de ces fentes, s'étendent des pl. appelées $p l$. génitales ou écailles génitales suivant qu'elles se trouvent sur le bord interrad. ou sur le bord rad. des fentes. Sur les parois des bourses, s’msorent de nombreuses petites phlandes isolées, les gol. génil. qui déversent teurs probluits dim les bourses. lieau de mer qui pénetre dans celles-ai par les fentes génit. entraine les produits sexuels, mais permet aussi des ebhanges gazeux avee le liquide de la cavité générale.

l.es ceul's lécondés domnent naissince à une larve très voisine du Pluteus des Oursins et appelée Ophiopluteus lig. 12, f) qui est munie de bras soutenus par des bagnettes calcaires. Certaines espèces sont vivipares, telle que l'Amphiura squamata de nos coteschez laquelle le développement s'effectue dans l'intérieur des bourses sans formation de larve libre, et les jeunes Ophiures sortent toutes formées par les fentes grenit. Il existe aussi une reproduction par lissiparité quion observe surtont che\% certaines espèces d'Ophiothela et d'Opliactis possédant tibras; le disque se coupe en 2 moitiés dont chacune emporte 3 hras, puis récrénère les 3 autres qui restent plus petits pendant un certain temps; ce phénomene sobserve notamment chez. une Ophiure de la Méditerranée, l'Ophiactis sirens.

Les bras des Ophiures présentent ehez l'animal vivant des mourements très vifs qui s'effectuent surtout dans un plan horizontal ; seules les Ophiures inférieures, dont les articulations vertébrales sont peu compliquées, peuvent infléchir leurs bras vertical. et les enrouler en tous sens.

Les Ophiures se nourrissent princip. de proies vivantes, de petits animanx qu'elles introduisent dans la bouche en les poussant a l'aide de leurs bras. Les espèces qui vivent en Méditerranée à quelques dm. de prol., parmi les Algues, telle que l'Ophioderma longicauda, penvent être capturées ì l'aide d'hameçons garnis de viande. En captivité, les Ophiures peuvent égal. être nourries avec de la viande. Elles sont extrêm. sensibles à l'eau douce dans laquelle elles meurent très rapidement et le plus souvent sans se briser.

La classification des Ophiures vivantes ef fossiles a élé remaniée récemment par Matscutoto, qui a divisé la classe en quatre sous-classes, principalement d'après les caractères du squel.; l'étude de ces caractères nécessite des dissections très délicates et je me contenterai de les rappeler ici d'une manière très brève. Je décrirai surtout les caractìres extérieurs des Ophiures qui sulfisent parfaitement pour la détermination.

S. Cl. Phrynophiurides. - Ophiures inférieures chezlesquelles le disque et les bras sont couverts par un tég. et les pl. brach. dors. sont nulles ou rudimentaires. Les boucliers rad. et les pl. génit. de chaque côté s'articulent par une simple facette ou par une saillie transv, de chaque plaque. 
S. Cl. Læmophiurides. - Les boucliers rad, et les pl. génit. s'articulent ensemble par une simple facette ou une saillie transs, sur chaque plaque; pl. brach. dors. bien développées; disque comvert d'un lég. plus on moins: épais cachant souvent les pl. sous jacentes qui sont munies de granules ou de piq.

S. Cl. Gnathophiurides. - Disque couvert de pl. fineset imbriquées: les boucliers rad. présentent chacun une fossette articulaire recerant un gros condyle formé parl'extrém. de la pl. génit. correspondante.

S. Cl. Chilophiurides. - Le disque est couvert de pl. plus ou moins grandes: les boucliers rad. et les pl. génit. sont réunis par deux condyles articulaires et une fossette sur chaque pl.

Lat détermination des Ophiures e-t plus délicate el un peu plus difficile que celle des Astéries. On examinera d'abord le mode de recouvrement du disque qui peut ètre nu, soit sur les 2 faces, soit sur la l'ace ventr, seulement, ou qui se montre garni de pl. minces, tantist inermes, tantot munies de piq. ou de granules: on évaluera la long. des bras par rapport au diam. du disque; ces bras ne sont ramitiés que dans une seule espèce française, l.Astrospartus arborescens; on étudiera la forme des pl. brach. les pl. brach. dors. manquent dans le g. Ophiomyra), la di-position des piq. brach. qui sont très longs on très petits, qui peuvent être appliqués contre les pl. lat. qui les portent, ou, au contraire, se dresser perpendic. à la direction des bras; les pores tentac. qui sont garnis ou non d'une ou de plusieurs écailles; entin on examinera la structure des pièces bucc. qui fournissent des caractères très importants pour la détermination et la classification des Ophiures.

\section{TABLEAU DES ESPĖCES}

1. Bras ramiliés un grand nombre de fois, très circonvolutionnes, les branches fortement entremélées et susceptibles de s'enrouler aussi bien dans un plan vertic, que dans un plan horiz, le tout formant une masse pourant atteindre 20 a $30 \mathrm{~cm}$. de diam.

- Bras simples, jamais ramitiés . p.

2. Le disque est couvert d'une peau molle et absolument nue, qui se continue sur les bras; les piq. brach. au nombre de 3 à 5 , sont courts et dressés perpendic. aux bras et leur base est reenuverte par le téz. : pas de papilles dent. : les dents ef les 
papilles buce. ollrent sur leur- bords me liange denticulée.

Ophiomyia pentagona p.

- La face dors. du disque est courerte de pl. quelquefois trè fines ou cachées par des granules mais toujours présentes: les piq. brach. dressés ou appliqués contre les bras sont dépomrus d'enveloppe legumentaire; les pl. brach. sont nues et leurs contours sont bien apparents.

3. Le disque est couvert de granules arrondis, très serrés, lisses. égaux, lormant un revetement contimu el trés rég. cachant complet. les pl. snus-jacentes qui sont tres fines.

- Pas de granules sur le disgue qui est convert de plolantot inermes tantrit munies de piq. on de pelits batomnets tres courts et comiques.

1. Les fentes rénit. sont simples et sétendent sur la lace ventr. du disque le long de la base des bras

les fentes gènit. sont dédoublées et de chaque coté des bras se montrent 2 léntes distinctes : l'une voisine du bouclier buce. el Jautre voisine du borl du disque. Les piq. brach. sont courts, mombreux une dizane environ. aplatis, appliqués contre les bras. Taille asser grande (diam. du disque 20 a 2.5 rm.

('phiode:"ma longicalla p.

D. Las de pap. dent.; les dents ont la forme de lamelles translucides: les granules qui reconrent tout le corps setendent jusque sur les pieces buce.; piq. brach. assez petits, aplatis, non appliqués: espèce de petite taille, rare. . . . Ophioconis forbesi p.

- Des papilles dent. formant un paquet bien apparent el cachant les dents qui ne sont pas lamelleuses: les granules du disque ne passent pat sur les pièces buce. Espece d'assec grande taille arec des bras allongés ef forts: les piy. brach. cylindriques el dressé sont creus

Ophiocomina nigra p.

6. Les papilles dent. très développées forment me masse vertic. ovalaire: les pl. orales sont grandes et les 2 pl. de cluaque paire sont séparées sur la ligne méd. par un orif. allongé et assez grand. Les piq. lorach. sont forts el allongés, dressés et munis de fortes denticulations: ils sont pleins el général. transprarents G. Ophiothrix

- Les pl. males smot petites et les 2 pl. de chaque paire sont conliguess sur la ligne med.. satms la moindre indicalion d'orif'.

Les pl, brach. dors. portent sur leur lace dors. de petits piq. Conlen rosere

Ophiothrit liitkeni p.

- Lar surf. des pl. brach. dors. ent parlatit. lisse et nue. 
la long. décroil jusqu au dernier; ces piq̣. sout an nombre de 7 . La face dors. du disq. porte des batomets el sourent des piq. : cenx-ci, lorsqu'ils existent, ne sont pas articulés sur des tubere. distincts. Coloration anse\% variée, souvent très vive.

Ophiothrix firgailis p.

- Les piq. brach. sont général. an nomb. de 6 et lenr long. angmente très rapid. à partir du 20" ventr. qui est beanconp plus long que le Ier, les 3", f" el 5" devenant très lomgs el atleignant presque la mème long.. le 6 " enfin est beancoup plus court. La face dors. du disque est tonjours munie de piq. fortement denticulés, el qui sont articulés chacun sur un petit maneton trée distinct. Couleur gris-jannatre, verditre ou rosé.

Ophiothrix quinquemaculata p.

?. Les pl. du disque sont extrem. lines, mais cependant recomnaissables au microscope sur des exempl. desséchés; les écailles tentac, au nombre de 2., sont très inég. : l'int., trè gramde, en forme de spatule élroile et allongée, se croise avee sat congénère sou- la face ventr. de la pl. brach, ventr. correspoudinte. Pliq. nombreuxel dresiér (i. Ophiopsila

- Les pores tentace sont tintot munis d'une écatlle unique, on bien de :2 ou plusicurs écailles, mais celles-ci restent loujours petites et subégales, jamais allongées.

10. Espéce petite el délicate avee des bras grèles; les piq. brach. tres courts, sont au nombre de 7 an plus. Ophiopsila aranea p.

- Espèce forte el robuste aree des bras épais el très allongés alleigrnant 20 a $25 \mathrm{~cm}$. de long.; me douzaine de piq. brach.

Ophiopsila annulosa p.

11. Face dors du disque munie d'écailles trè fines difficiles a distinguer: les piq. brach. allongés el assez cassants, sont creux et dressés perpendic. a laxe du bras; les boucliers rad. se contimuent vers le centre du disque par autant de cotes saillantes, convertes de petites pl. portant des bàtomets coniques, très courts et ruguenx . . . . . . Ophiacanthu setosa p.

- La fice dor.s. du disque est couverte de pl. très distinctes el n'offre jamais de cotes rad. saillantes; les piq. brach. sont courts et plein:

1?. Expeces rolustes avee des hras rigiden: disque comert de pl. fortes et très solidement unies; les premières paires de pores tentac. portent plusicurs écailles de chaque coté : les pl. génit. offrent sur leur bord ext. ou interrad. une rangée de prapilles qui se continuent sur la lace dors. du disque de chaque coté de la base des bras, où elles s'allongent en petits piq. formant une sorte de peigne, appelé le peigne radial G. (Ipliura. . . . . . .

Espèces d'asic\% petite taille et délieates; bras souples, sinueux et 
allongés: les pl, du disque sont petites: le nombre des écailles tentac. n’est jamais supérieur à 2.

13. Espece très robuste et de très grande taille, le disque pouvant atteindre $30 \mathrm{~cm}$. de diam.; il existe des dépressions entre les 6 ou 8 premières pl. brach. ventr. . . Ophinra lacertosa ip. (1)

Espèce plus petite, le diam. ne dépassant guère $15 \mathrm{~mm}$; il nexiste pas de dépressions entre les premières pl. brach. ventr.

Ophiura albida p.

1'. Entre les pl. orates et adorales, et de chaque coté, se trouve intercalée une pl. supplémentaire plus grande que les pl. orales, et qui peut ètre considérée comme représentant une papille bucc. ext.; très rare. . . . . . Paramphinra punctata (p.

- Les pièce bucc, oflrent une structure normale, c. à d. comprement daus chaque interad. un bouchier bucc., une paire de pl. adorales et une paire de $\mathrm{pl}$, orales conliguës aux précédentes, avec une à 3 papilles buce. de chaque còté .

15. Les 2 pl. orales supportent ensemble une seute papille buce. impaire termin.; il existe en plus une papille buce. lat.; G. Ophiactis?. . Lij

- Les 2 pl. males supportent chacune une papille termin.; il y a :2 papilles buce. lat. ce qui fait, en tont, 3 papilles buec, de chaque coité.

16. Espece a 5 bras vivant toujours a me certaine prof.; couleur ponrpre-brun, brun, rouge ou marron . . Ophiactis ballip.

- Espèce tittorale, à 6 bras, se multipliant par lissiparité ; couleur verdatre ou jaunatre. . . . . . . * Ophiactis virens p.

17. Les 3 papilles buce, sont contiguës, les 2 int. assez petites, la papille ext. très élargie et rectangulaire; ces 3 papilles se suivent en une rangée continue et très rég. et en rejoignant leurs congénères, elles ferment complèt. la bonche. Disque petil, ne dépassant gruère í $11 m$. de diam.: boucliers rad. contigus; bras courts et très fins; : écailles tentac. La seule espèce connue dans nos mers est vivipare . Amphipholis squamata p.

- Les papilles buce, ne sont pas contiguës et ne forment pas une rangée régul. el continue susceptible de lermer la bouche; les bras sont allonges

18. Le disque est pourvo de piq.. an moins a sa périphérie sur la face dors. ainsi que $-m$ la face ventr.; il esiste zécaille- tentace a la base des bras et celles-ci se contintent sur un certain nombre d'art., mais finisent par disparaitre: les pl. brach. ventr. somt cammelées. Bras extrèm. longs. Ophiocentrus brachiatus p. 85

- Aucune trace de piq. sur les pl. dors. on ventr. du disque (i. Amphiura]

19. Pas d'écailles tentac, ; lace ventr. du disque en grande partie nue; le ze pig. brach. vente. ont muni a som extrém. de 2 crochets 
Iransv. on dil qüil est bihamule). Imphiura filiformis p. 81)

2 écailles tentac.; face ventr. du disque couverte de pl. sur toute

son élendue : le 2e piq. brach. ventr. est identique aux antres. . 201

?11. La face dors. du disque othe en som eentre mue roselle de 6 pl. prim. plus grandes que les autres: une centr. el is rad.; les piq. brach. sont an nombre de ja ti sur des echant. dont le disque a ?

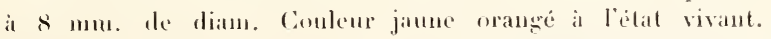
Ampline chiajei (p. is

- 11 nexiste pas de roselte prim. sur la face dors. du disque dont le centre ollre seulement une pl. un peu plus grande que les autres; les piq. brach. sont nombreux ( 8 i 10 sur des exempl. dont le disque a $u$ diam. de 6 mm.) ; les brats sont relativ. très minces, plus minces que dans l'espèce précédente. Couleur grise à l'état vivant

Amphiura medilerranea p. is

\section{S. GL. PHRYNOPHIURIDES}

\section{F. GORGONOCEPHALIDÆ DödERLEIN.}

Le disque et les bras sont couverts d'un tég. épais, revêtu de granules et eachant complètement les pl. : les boucliers rad. ont la forme de cotes saillantes. allant du centre du disque à la périphérie. Les dents et les papilles buce. ol dent. sont toutes de méme forme et spiniformes. Dins la seule espece de nos mers, les bras sont ranifiés un très grand nombre de fois.

\section{(i. ASTROSPARTUS lömRlais.}

Le disque est épais, pentagonal, avee les colés plus ou moins excivés; il donne naissance "ै " bras qui so divisent dies leur base en 2 branches, puis chaque branche se divise de nouveau cn 2 autres qui se divisent elles-mêmes, et ainsi de suite un grand nombre de fois, en meme temps que les ramifications devienuent de plus en plus fines. Ainsi se forme autour el en dehors du disque un ensemble de ramifieations encherêtrées les unes daus less autres el qui a élé comparé à une tète de Méduse, d’où le nom donné à la famille. La face veutr. du disque oflre, dans les espaces interrad., des pl. plus ou moms apparentes, et la pl. madrép., unique, se trouve placée entre les pl. adorales et ees pl. interrad. ventr. aceessoires. Ies pirf. brach. font compliol. défaul : il nexiste, sur la face vente. des 
bras, yue de petites papilles placées au voisinage des pores tentae. : ces papilles restent tres felites ef dialleurs peu nombrenses sur les ramifieations de grosse ch de morente laille, mais, sur les plus lines, elles deviement relativement plus afprarentes et se framsforment en erochets. Les ant. suecessifs des bras sont indi'fués par de petiles bandes transr. ou les granules sont remplacés par de petits erochets.

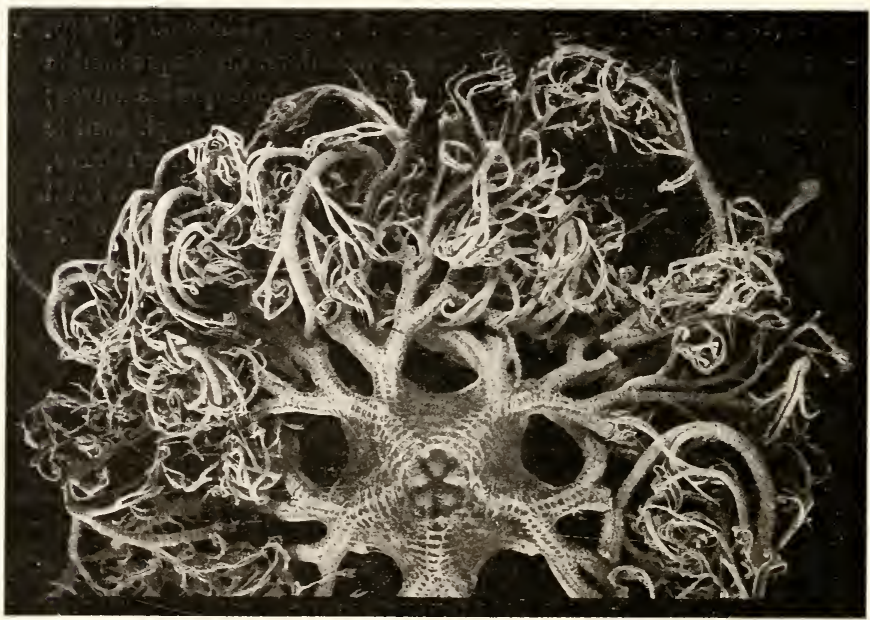

Fis. 43. - Astrospartus arborescens; face ventrile: $\times 1 / 2$.

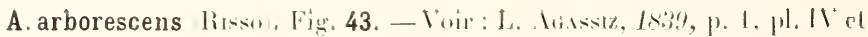

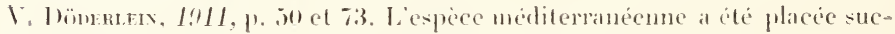
ecsivement dam les s. Euryale, Gorgonocephulus ef Astrophyton; Dömons l'a rangée dans le w. Astrospartus en raison de la siluation de la pl. madrép.

Lesechant, sont tres grands : le disque peut atteindre 5 a $6 \mathrm{~cm}$, de diam. ef les ramifications des bras convent, dams lems ensemble, nu espace de

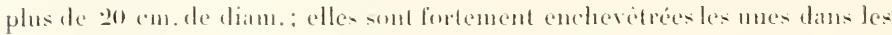
autres et enroules dans lous les sens. les bras sont bifurqué dés leur orisine sur te disque, et quand on resarde l'Ophiure par en haut, on n'en aperçoit pas le commencement, mais sculement les 2 premicres bifurcations qui sont courtes et egales, tandis que les snivantes sont plus longues; ces bifurcations se succedent en fournissant alternat. a droite et à gauche, des branches dont la larg. dimimue progressivement et on en peut

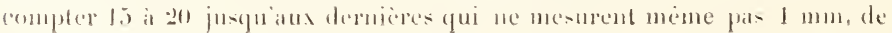


larg. Les pores tentac. de la premiere pare sont beancoup plus grands que les suivants el ils oflrent sur leurs bords des granules plus grosiciers que les voisins, mais ils sont dépourus d'éailles lentac. eelles-ei napparaissant que vers le $6^{\circ}$ on $7^{\circ}$ art. Il y en a d'abord une seule, puis -2 el ce dernier chilfre se maintient sur foutes les ramificalions: partors ependant il en existe 3: ee sont de petits piq. 22 lois plus longs que lareges, portant a leur extrém. 1 ou 2 pointes coniques el hyalines. Entre les pores tentac. successifs, la lane ventr, des bras presente sur les 2 on 3 premières bifureations. des dépressions franss qui correspondent aux art brach.

la couleur est grise chez limimal vivant, elle devient plus elare dans lialeoul.

I: 1. arborescens est spécial it la Méditerranéc. Sur nos eotes de Provence, cette espèce vit principalement sur les fonds rocheux, vers $50 \mathrm{~m}$. de prof., d'ou les pécheurs la rapportent parfois acerochée i leurs filels; on peut la trouver à la crite, rejetée par les tempretes. On la connail égal. a Napless, sur les eites de sicile, ete.

\section{F. OPHIOMYXIDÆ LsexqMax.}

Le disque et les bras sont reconverts diun leig. assez mince: les bencliers rad. sont rudimentaires et les pl. brich. dors. font defaut: les bras sont toujours simples. Wune maniere générale, lorganisation est inféricure.

\section{G. OPHIOMYXA MüßLek et Thoschel.}

Le disque est mou et charnu, recouvert d'un tég. complet. dépourru de pl. et qui s'étend sur les bras en recourrant la base des piq. brach. ; il existe toutefois quelques écailles sur les bords du disque; les pl. brach. rentr. sont risibles. I.es pièces buce. sont bien développées : les pl. adorales sont trés grandes el fournissent, en dehors, un lobe qui separe le bouclier buce de la première pl. brach. lat. I.es papilles buec. et les dents ont lit forme de lamelles aplaties et denticulees sur les bords. Les pores tentare sont nus.

0. pentagona Mülefr el 'Troscule. Migr. 44. - Voir : Müller el Troschel, 1842 , p. 108 , pl. IX. fig. 3.

Le disque est assez grand, penlagonal, deformable chez l'animal vivant;

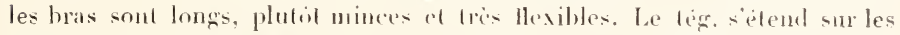
pièces buce. el ne laisse à mu que les papilles buce. qui ullrent une colotation blanche tranchant sur le reste du corps lortement colné en brun. Les fentes grénit. natleignent pas le bord du disque ef offenl sur leur bord int. une série de petites pl. qui rejoignent le bouelier bues. les pl. brach. vente. plus ou moins visibles it travers le tieg. sont pentagonales, un peut plus longues que larges aree le bord dist, echancré. Les piq. brach, sont 
d'abord an nombre de 5 , puis de 1 ; ils restent assez courts et leur long. augmente du premier ventr. an demier dors. dont la fongr. nalleint meme pals celle de l'art.

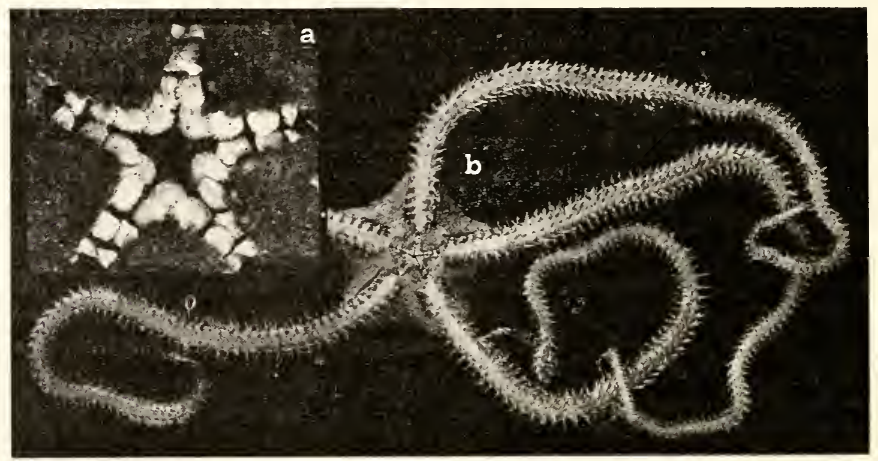

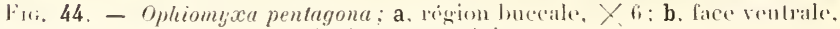
legrement réluite.

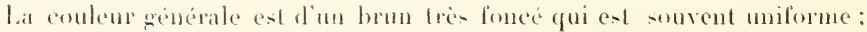
parfois il existe sur te disque de pelifes laches blanches el ces laches se continuent - ne la face dors des bras on elles se developpent davaulage el

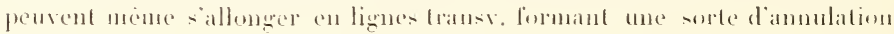
irrézulul.

LiO. pentagona ont asse\% cotimune daus la léditerane oit on la trouve a la cote sons les pierres, contre les rochers ou les parois des jetees, parmi les Mlgnes. Elle peut descentre a une ecrtaine prof. et se montre associe anx Holothuria forstiali el polii. Sur nos eciles de Provenee, elle as liréquente en bromindo ", el elle pent desermele beaucoup plus bas jusquäi 100 m. : on la

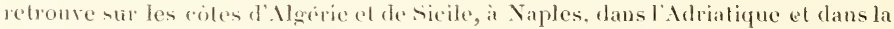
ner Égée. Elle a été considérée longtemps comme propre à la Jéditerranée, mans elle até reneontrée sur les eotes d'lfrique, an eap Blane ef aux iles du Cap Vent: toutelois, elle ne parait pas remonter sur nos cótes océaniques. 


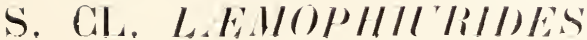

\section{F. OPhiaGanthidÆ Perrilir.}

Le disque est convert de petiles pl. plus ou moins eachées dans le tég. ef portant des granules, des tuberc. ou de petils piq.; il existe des dents et des papilles buce, mais pas de papilles dent. Les bras sont ordin. moniliformes, les pl. lat. ćlant très élargies ot épaissies dans leur rég. dist. qui porte les pirf.; ceux-ci sont grands el très déreloppés, ordin. transparents el denticulés. Les pl. brach., dors. et rentr. sont petiles. largenent séparées par les pl. lat. qui sont contiguës sur les lignes méd. dors. el rentr.

\section{G. OPhiacantha Iï̈llen et Troschel.}

Les faces ventr. et dors. du disque sont couvertes d'un tég. dans lequel sont cachées des pl. très pelites, très minces et imbritpuées, yui ne s'aperçoivent guère que sur les échant. desséchés. les houcl. rad. se prolongent en còtes étroites et proéminentes, munies de granules rugueux yui se montrent aussi, mais moins développés, sur les rég. interrad. Les papilles buce. sont coniques et pointues. Les piq. brach., longs et divergents, sont minces ef creux.

0. setosa, Müllfer el Truschel. Fig. 45. - Voir: Kinmer, 189, p. 57. pl. VIIl, lig. 37 et 38 .

Le disque peut alleindre 1:2 mm. de diam. : il eat pentagonal chez l'animal vivant: les bras sont minces el lenr long. égale ś a lo fois le diam. du disque. Lat face dors. du disque est courerte de très lines écailles, visibles surtout vers la périphérie: elle ollre j paires de cotes rad, saillantes, à lextrém. desquelles se tromvent les très petits boucliers rad., qui portent de petits lubere. ou granules rugueux qu'on refrouve anssi sur les parties voisines. Les papilles buce. lat., ordin, au nombre de 3, partois de 4 , sont coniques, pointues ef rugueuses. Jes pl. brach. dors. sont triangulaires et bombées, les ventr. sont pentagnonales. Les piq. brach. au nombre de 7 et parfois de 8 sont garnis de fines denticulations. L'ecaille lentac. est pelite, conique el rugueuse.

La couleur à l'étal vivant est brune, brun violacé ou gris jaunâtre; elle se conserve en partie dans l'alcool. 


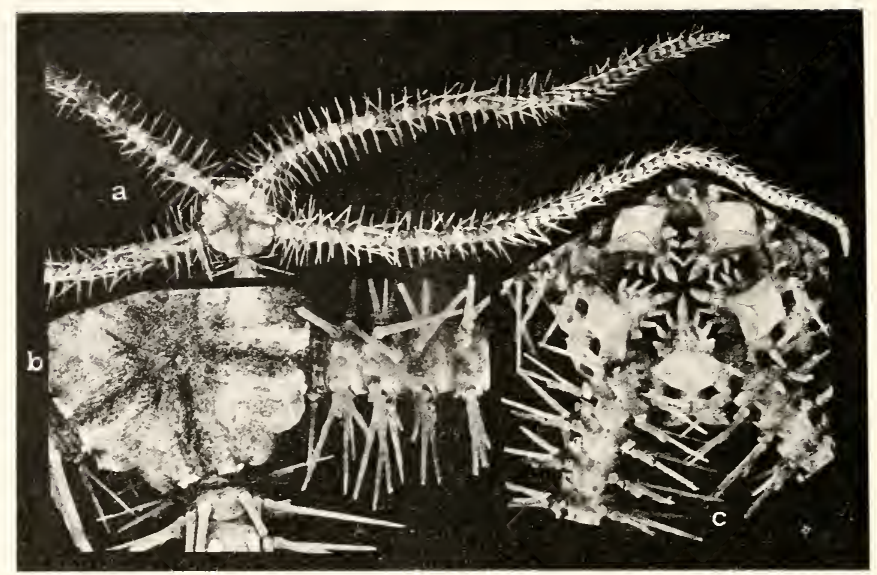

F16. 45. - Ophiarantha setosa; a, face dorsale, grandeur naturelle; b, face dorsale, et c, face ventrale, $X$ 't environ.

lin. setaia se trouve principal. en Mediterrance. Sur nos cotes de Provence, on la reneontre sur les fonds rocheux, rers io i : $50 \mathrm{~m}$. ; on l'a signalée à Naples, à Palerne et sur nos cotes d'Mlgérie, toujours a une certaine prof.: elle pent descendre jusqu i quelques centaines de $\mathrm{m}$. On a cru pendant longtemps quelle était propre à la léditerranée, mais les expéditions réeentes l'ont rencontrée dans le golte de Giascogne, sur les crites l'Espagne et d'Afrique, is des prof. variant de 60 it $653 \mathrm{~m}$, el atteignant meme $1,480 \mathrm{~m}$.

\section{S. CL. GNATHOPHILRIDES}

\section{F. OPHIOTHRICHIDÆ Luxgraxx.}

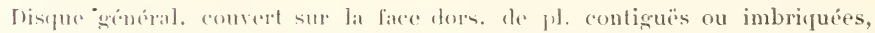
pouvant faire defant sur la wolr. soment an partie nue. Elles pertent habit. des bitonnets ou piq. plun ou moins allongris. Boucliers rad, ordin. très grands. Les 
2 pl. orales d'une paire, tris forles, se touchent seulement par leur extrém. prox. laissant en ariere un espace vide très apparent. Pas de papilles buce. : les dentaires, nombreuses, en un groupe ovale. Pl. lorach. dors. entières on divisies, lespiq. brach. tantot longs, hyalins et denticulés, fantot courts et opaques.

\section{G. OPHIOThRIX Yïr.ter et Thoschif.}

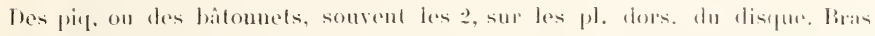

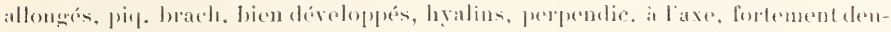

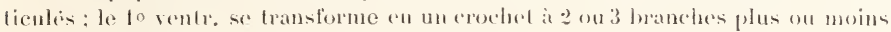
Join du disque, Pl. brach, dors. grandes, entieres.

0. lütkeni Mrrulf-Tuomsox. Fig. 46. - Voir : Kintuler, 1909. p. 201, pl. XXIX, fig. \&, y el 10.

le diam. du disque peul mesmer 1.j a 20 mm. el les bras arrivent a 1.50 $10 m$, de longr. le disque est arrondi, plus on moins proéminent dous les espaces interrad.; les boucliers rad., très grands, offrent a leur surf. un eertain nombre de petils piq. grêles et comrts: les intervalles eutre ces boucliers portent des pl. munies ehacune d'un piquant fort el allongé. Les pl. brach. dors. sonl grandes, 111 pen imbriquées el elles offrent toujours à leursurface, dans les exempl. adultes, un certain nombre de tries pelits piq. fins, analogues à ceux des boucliers rad., el plus nombreux an voisinage du bord dist. Les piq. brach. sonl au nombre de s et leur long. augmente assez régul. du ler au $^{*}$, qui ent égal

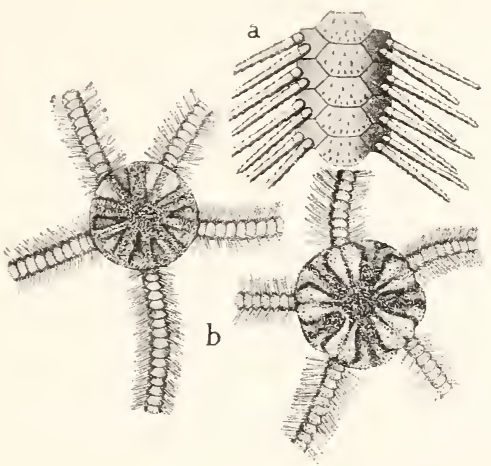

Fis. 46. - Ophiothrix lätkeni; a, portion de la face dorsale d'un linas, $X ?$; b, face dorsale du disyue et de la partie voisine des bras. à 2 art. 12 ; les 2 premiers piq. ventr. sont grèles el le dernier dors. est souvent très petit. Ces piq.. transparents, sont munis de denticulations forles el serrées.

Les indiv, vivants offrent une teinte générale gris rosé ou gris verditre, ou encore rose verdatre, et les bras sont d'une teinte uniforme ou annelés de griset de rose. Les grands exempl. présententordin. sur la l'ace dors. du disque des bandes rouges ou pourpres dirigées suivant les rad. on les interrad. el parfois les 2 i la fois; ces bandes encadrent sourent les boncliers rad, Ces colorations sont conservées plus ou moins complèt. dans l'alcool, 
L'O. lütkeni se reconnait facilement à sa grande taille et surtout aux petits piq. que portent les boucliers rad. et les pl. brach. dors. C'est une espèce propre à l'Atlantique. Elle parait très fréyuente dans le golfe de Gascogne, sur le plateau continental à partir de $100 \mathrm{~m}$, dans le sable ou dans les graviers, au milieu des coquilles brisées, ete. D'une manière générale, l’o. lütkeni se trouve à des prof. où l'o. fragilis ne penètre pas.

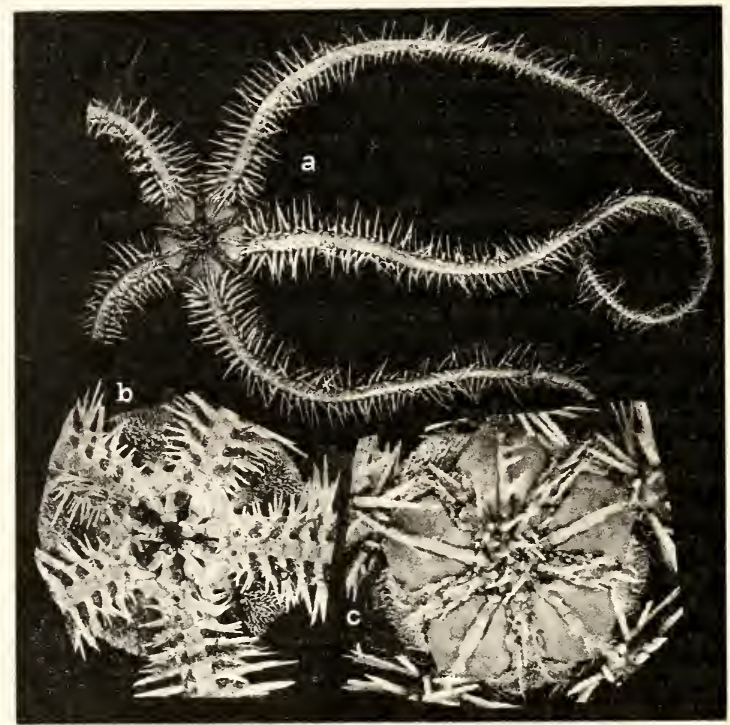

FIG. 47. - Ophiothrix quinquemaculata, a, face dorsale, légèrement réduite; b. face rentrale, et c, face dorsale; $X 3$.

0. quinquemaculata (Delle Chlase). Fig. 47. - Voir : Lütkes, 1869, p. 52 et 101; Kuemer, 19:1 (O. quinquemaculata ; lisso, 1893, p. 7, pl. 1,

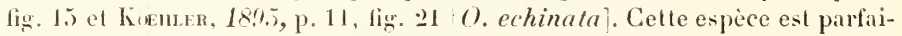
tement caractérisée el facile à déterminer. Le nom qu'elle doit porter at subi certains flottements, mais jestime qüil y at lieu de lui appliquer la dénomination choisie par l'antem qui l'a netlement distingnée el décrite pour la première fois el qui est lürss. J'ai pu étudier les types de cet anteur et rectifier le nom d' $O$. echinata qui avait été appliqué longtemps et à tort à celte espèce (1).

(1) Les échant. d'O. echinata et d'o. quinquemaculata de Lürkes, qui se trourent au Musée de Copenhague, mont été obligeamment communiqués par mon excellent ami 
L'ensemble de l'animal est assez robuste; le diam. du disque varie entre 10 et $15 \mathrm{~mm}$. et la long. des bras atteint 8 i 10 fois ce diam. La face dors. du disque se fait remarquer par les très grands boucliers rad, triangulaires, plus longs que la moitié du rayon du disque et toujours nus: l'espace laissé libre entre ces boucliers est occupé par de petites pl. allongées radiairement, à contour ordin. bien apparent, dont les plus grandes portent chacune un tuberc. arrondi sur lequel s'articule un piq. plus ou moins développé; les plus petites portent seulement un batomnet qui surticule égal. sur um tuberc. Les piq. du disque sont lins et allongés, poinlus, et mesurent 2 a 2,5 mm. : les batomuets sont coniques et se terminent par quelques pointes minces, acérées, parallèles, au nombre de 3 à 5. Jes piq. du disque sont plus développés el plus nombreux sur certains indiv. que sur d'autres, mais ils existent toujours ainsi que les batonnets, et je n'ai jamais rencontré d'exempl. che\% lesquels l'une de ces ? formations fit défaut.

Les bras offrent sur leur ligne médiane dors. une carène arrondie. Les pl. brach. dors. sont en éventail, et l'anghte dist. se relève en un petit hec arrondi et peu saillant. Les piq. brach., an nombre de 6 en principe, offrent la disposition caractéristique suivante : le $1^{\text {er }}$ est très court, le $2^{\mathrm{e}}$ est plus long, et égale à peu près l'art.; le $3^{\circ}$ est beaucoup plus long et il dépasse 2 art. ; les 2 piq. suivants sont encore plus longs, ils dépassent 3 articles et souvent même atteignent la long. de 1 ; enfin le dernier est beaucoup plus court. Ces piq., incolores et transparents, sont munis sur tonte leur long. de dents assez fortes, très pointues, serrées et très régul. disposées. Le prenier piq. ventr. se transforme en un crochet à 3 branches à une grande distance du disque. Les denticulations sont plus fortes et serrées à la partie termin. des piq. ; ceux-ci s'amincissent assez rapidement jusqu'ì leur extrém. qui est un peu tronquée, au moins sur les piq. lat., car le piq. dors. seul est pointu.

Les exempl. de nos còtes de Provence sont d'un gzris rosé, moses ou moseverdàtre sur la face dors.: les bras offrent tankit la meme coloration uniforme que le disque tantiot des ammulations pourpres irrégul, disporés tons

M. le Prof. Mortevsen, et j'ai pu établir l'une maniepe précise que l'". quinquemaculata correspondait bien à la forme qui est si eommume en Vétiterranée à partir le 30 à $60 \mathrm{~m}$. tandis que l'O. eshinata représentait une forme littorale. En 1893, Rrsso avait donné le nom d'O. echinata à l'O. quillquemaculata de Lüthes el il indiquait d'une manière très précise la disposition des piq. qui est caractiristirpue dans cette espréce de telle sorte que n'ayant pas en l'oceasion de voir les types do Lütren et supposant que Rrsso avait pu voir les originaux de Deste Chuse, f'arais, suivant l'exemple de l'auteur italien, appelé $O$. echinata l'Ophiure on question clans mon mémovire de 1893 et dans quelques autres qui le suivirent. J'ai rectifiè cette synonymie dans un mémoire actuellement sous presse à Washington sur les "Ophiures recueillies par l" "Albatross " aux iles Philippines $\bowtie$. 
les 3 ou 1 art. : les échant. en alcool sont décolorés, mais les annulations des bras sont général. conservées.

L'O. quinquemaculata est très commune en Véditerranée mais à partir d'une prof. de $40 \mathrm{~m}$. seulement; elle est extrèm. répandue dans les fonds vaseux du large où elle doit former par place de véritables tapis d'où elle exclut les autres animaux : jai sourent vu des pêcheurs en rapporter dans leurs filets quelques centaines de kilos. Ville ne parait pas pournir atleindre de grandes prof., mais je n'ai pas de précisions à cet égard.

0. fragilis (Anmbiıнu). Figr. 48 el 49. - Voir: Risso, 184., p. 6; Kiofulrar, $18 \% 5, \mathrm{p}$. 13. Les caractères de cette espèce sont extrêmement variables et il est diflicile d'en donner une description qui śapplique aux innombrables formes qu'elle alfecte; divers auteurs ont même cru devoir la diviser en un certain nombre d'espèces, 4 ou 5 pour les uns, et jusqu'à 7 ou s pour d'autres. J'estime, pour ma part, qu'il ne s'agit que d'une seule et mème espèce, très polymorphe, dont les variations tiennent aux localités et aux profondeurs el sont reliées par de nombreux termes de passage. On peul grouper ces formes rariées et sariantes en t́ variétés principales dont je résumerai plus bas les caractires el que jappellerai echinatu, pentaphyllum, lusitanica el abildgarrdi.

Les dimensions de l' O. fragilis varient beaucoup; les échant. les plus communs surnos côtes el qui répondent à la var. echinata, sont de petite taille, le disque ne dépassant pas 7 à $8 \mathrm{~mm}$. de diam. et les bras $40 \mathrm{~mm}$. de long. ; dans la var, pentaphyllum, le disque peut atteindre 12 a $14 \mathrm{~mm}$. de diam. et les bras ont 70 a $80 \mathrm{~mm}$. de long. ; dans la var. abildgaardi, le disque est très grand mais les bras sont courts. Ces bras se brisent très facilement soit lorsquion saisit l'Ophiure à la main. soit lorsqu'on la plonge dans un liquide conservateur.

Les boucliers rad. sont ordin. de grande taille et nus; le reste de la face dors. est occupé par des piq. ou des batonnets spinuleux, ou le plus souvent par les deux formes à la fois. La face dors. des bras est plus ou moins earénée ; les art. successifs sont plutôt courts. Les pl. brach. dors. sont assez. grandes, en forme d'éventail, avec l'angle proxim. plus ou moins saillant et formant sonvent un hec assez accusé, en arrière dnquel se tronve une petite proéminence arondie, le tout déterminant une carène qui s'étend sur le milieu de la face dors. des bras. Les pl. lat. portent ordin. 7 piq. vitreux, transparenta, dout la longh. angmente d'une façon très lente el très progressive du $1^{\text {pr }}$ ventr, qui est très court au $5^{\circ}$; ce dernier et le piq. suivant sont les plus longs, puis le te devient plus court. Dans les grands échant., il y a parfois s piq., mais l'allongement reste tonjours tres progressif du tor an f $^{\circ}$, lequel natteint quere que la long. de l'art.: les piq. snivants mesurent à peu priss un art. et demi sur les petits exempl. et 2 dans les plus grands. Ces piq. sont transparents, aplatis, tronqués à l'extrém. plus on moins échinulés sur les bords suivant les var. Le premier ventr, se lransforme assez. vite en crochet. 
Ainsi que je l'ai dit plus haut, les Ophiothrix de nos cotes offrent de trìs grandes variations; c'est surtout pour la commodité de la description qu'il est utile de distinguer des variétés, mais ces variétés sont reliées par de nombreux termes de passage el il est souvent difficile d'établir entre elles une ligne de démareation. Comme toutes les Ophiolhrir liltorales de nos eaux sont des O. fragilis, on peut étre certain de ne pas faire une erreur de délermination en leur donnant ee nom; ceest l'alliare des spéciatistes do reconnatre les var. d'en résume néammoins les carateres principaus.

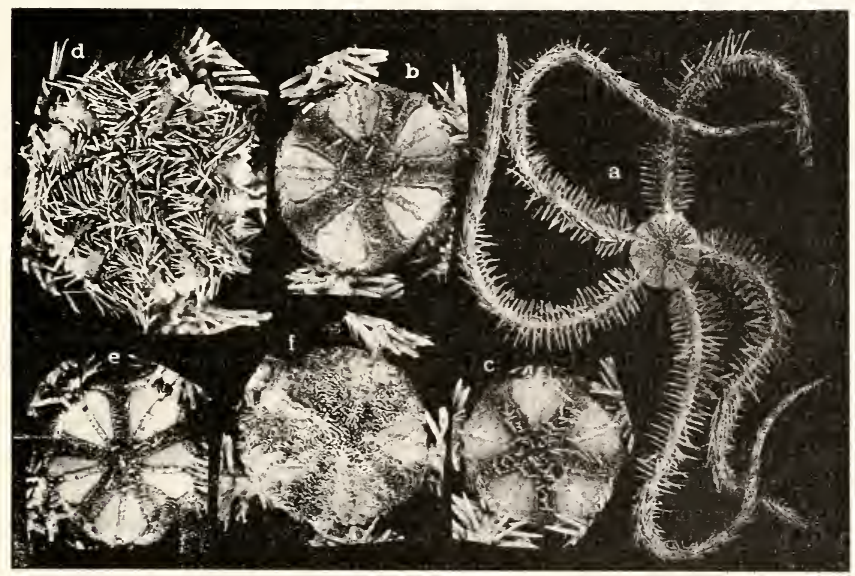

Fig. 48. - Ophiothrir fragilis var. echinala; face dormale: a, schantillon de cette légirement grossi ; b et c, échantillons de Cette: d, de la baie du lévrier; e, de lioseoff; f, de (Suiberon.

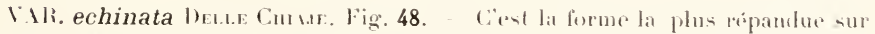

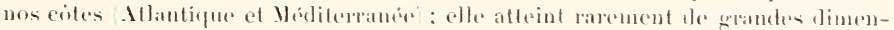

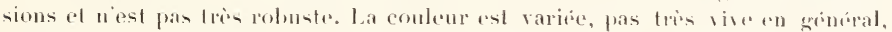

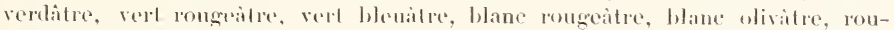
geatre et mime rouge ef se maintient dins lalcool. La pointe relerie que forme l'angle dist. des pl. brach. dors. e'st souvent marquie par un petit point blane; les piq. offrent une couleur voisine de eelle de la face dors. des bras, mais plus claire et ils sont transparents; ils sont plus ou moins divergents et disposés en éventail; la long. égale environ une fois et demie la larg. des bras. Ceux-ci offrent parfois des annulations plus claires el plus foncées. Dans les formes les plus communes (a, b, c, e), les boucliers rad., assez grands, sont nus; les espaces interrad. sont couverts de bilonnets courts, terminés par quelques spinules coniques, pointues, assez courtes, entrenélées de piq. plus ou moins allongés et munis de quelques denticules. De cette forme movenne des modifications 
peurent se faire suirant 2 sens opposés: ou bien les bâtonnets se développent aux dépens des piq. qu'ils finissent par exclure complèt. de la face dors. du disq. f, ou bien ceux-cı prennent un très grand déreloppement et aequièrent la prépondérance (d). L'on peut trouver dans les mêmes localités, sur nos côtes de Yormandie ou de Bretagne, daus les canaux de Cette, ete, des indir. offrant un mélange de bâtonnets et de pị. associés ì d'antres chez lesquels les bàtonnets existent seuls; ces bàtonnets peurent d'ailleurs s'allonger et ressembler beaucoup ì de petits piq. Les boucliers rad. peuvent aussi porter de petits bâton nets. mais cela est rare. Les formes dans lesquelles les piyuants dominent sur la face dors. du disque et même font disparaitre les bâtomnets sont rares sur nos cótes; c'est à elles que le nom d'o. alopecurus avait été particul. appliqué.

VAR. pentaphyllum Luxgm. Fig. 49, b, et c. - Cette var. a été décrite autrefois par Fon l'O. echinata: le diam. du disque alteint 10 et $12 \mathrm{~mm}$. et les bras, forts et allongés, ont de 70 à $80 \mathrm{~mm}$. de long. Les boucliers rad. sont nus, plutôt granels et très apparents; les espaces qu'ils laissent libres sont occupés par des bâtonnets accompagnés de piq. Dans les formes les plus robustes, ces bat. sont gros, coniques, courts et terminés au sommet par quelques spinules; ailleurs ils sont plus allongés: les dimensions des piry. varient égal. Les piq. brach. sont aplatis, fortement denticulés, rapprochés les uns des autres et assez longs : lour long. igale 2 fois à 2 fois 1 ' la larg. dubras. La couleur est toujours vive et brillante et le rouge domine orlin. : lan tôt la face dors. du disque est uniformément rose, tantôt elle est blanchìtre arec des taches rouges plus ou moins nombreuses; la face dors. des bras est rouge et peut offrir des annulations; les piq. sont peu ou pas colorés: ailleur's la couleur générale est bleue, tantòt bleu foncé, tantôt bleu grisître ou bleu rosé, uniforme ou arec des taches plus claires des cercles, ele. Les colorations sont assez bien conservées dans l'alcool.

Celte forme est très répandue dans le Pas de Calais, ¿t Wimereux, à Boulogne, au Portel, etc., dans des stations tout à fait littorale où elle remplace la var. echinata; c'est olle qui domine sur les côtes d'Angleterre: elle peut descendre ì des prof. de 10 i $20 \mathrm{~m}$. sans que ses caractères varient beaucoup. Sur les côtes de Bretagne et dans l'Atlantique, l'O. pentaphylium existe égal., mais toujours à une certaine prof., tandis que les échant. littoranx appartiennent i la var. echinata.

VAR. lusitanica Luxgux. Fig. 49, d. - Celle rar. rappelle les indiv. d'o. echinata, dont la face dors. du disque est couverte de batonnets sans jiqf. Le disque est pentagonal el les rég. interrad. sout proéminentes. Les boucliers rad. sont plutiot petits, ordin. nus et tout le reste de la face dors. est couvert de petits bàtounets arrivant tous exactement à la même haut., ayant la mène taille ef terminés chacun par une couromne très régul. de pretites spinules pointues et lígèr. divergentes, dont le nombre est le plus souvent de 3 . Les bras ne sont pas très longs et leur long. égale à peu près 4 fois le diam. du disq. ; les piq. brach. sont rapprochés, étalés horizont. et its sont plutît courts: ils sont épais, un peu opapues, aplatis et garnis de denticules. Cette var." reste littorale. La couleur est uniformément rosée. grise ouverditre et n'olfre jamais la viariété ou l'élégance qui existe dans les 2 var. précédentes; de plus clle passe complèt. dans l'alcool.

V.1li. abildgaardi Kinnm.in. Fig. 41, a. - La forme générale est trapue et ramasstó. T.e disque est épais, charnu, plutôt mou et il déborde largement dans les 


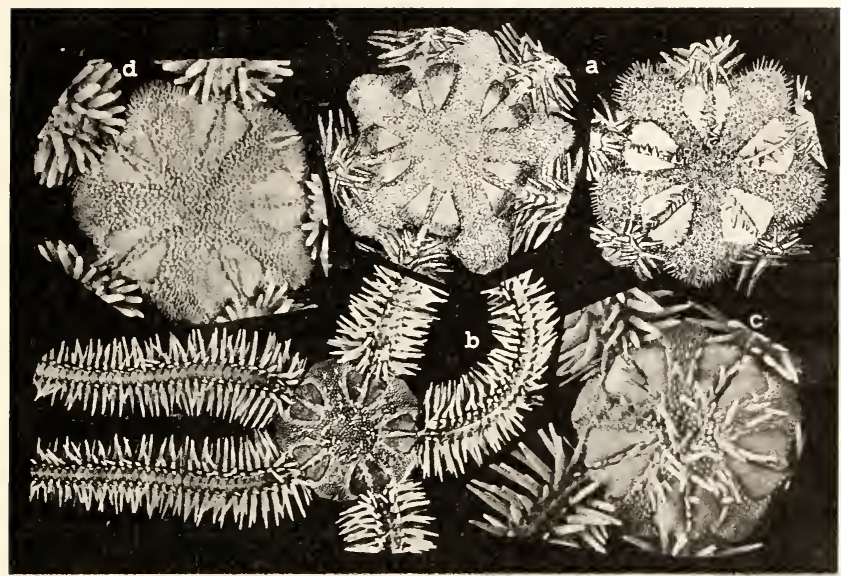

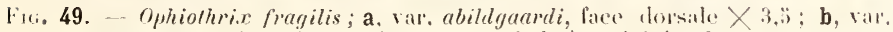

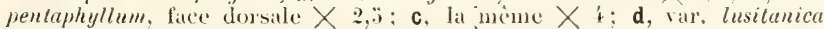
$\times 6$.

rspaces interbrachiaux: les bras sont assez larges mais plutot courts ; les bouclicrs rad. sont asser protond. enfoncriset plutot petits. Le disifue pent atteindre $20 \mathrm{~mm}$. de diam.; tantit il est uniformement courert de pelits bitomets courts, spinuleux, tantit de vrais picl. s'ajoutent aux bitonnets, mais ils restent toujours peu nombreux et assez épais. La carene des bras est peu marquée. Les piq. brach. sont assez gros, courls, un peu opaques et les denticulations pas très fortes.

Cette forme, décrite autrefois par Abutbgand sous le nom d'o. fragilis, est très répandue dans toules les mers $d u N$. ou elle est littorale; elle est commune sur les côtes de Norrigre ou elle presente ses caractères les plus typiques: on rencontre parfois dans la Manche et dans l'Atlanlique, a une ecrtaine prof., des O. pentaphyllum ì disque épais et gros, à bras courts, qui se rapprochent beaucoup des 0. abildgaardi du N. (1).

(1) Jattirerai l'attention sur un point spreicial au sujet des Ophiothrix. 11 n'y a guert lieu d'utiliser les carartères anatomiques pour la détermination des espèces de ce $\mathrm{g}$. ; cette étude est d'ailleurs à peine ébauchẻe chez les Ophiures, et une tentative qui a ètè faite autrefois dans ce sens par un naturaliste, n'a pas été très lıeureuse : je veux parler d’Apostolı́s qui, dans un mémoire d'ailleur's riche en erreur's, avait avancé que les vésic. de Poli n'existaient pas dans la var. echinata (qu'il appelait versicolor), et se trouvaient seulement chez l'O. quinquemaculala ('ju'il appelait rosula) - ce qui est faux. Toutefois, je erois bon de rappeler ici les differences que l'on observe au début du développement de nos ophinthrix littorales, on sait, en vitet, que les Pluteus trouvés en 


\section{F. AMPHIURIDÆI I}

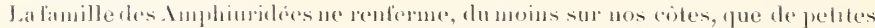

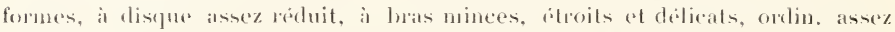

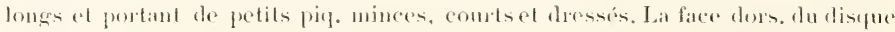

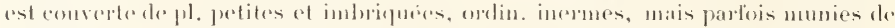

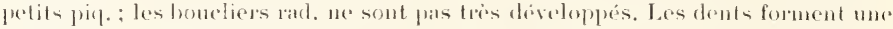

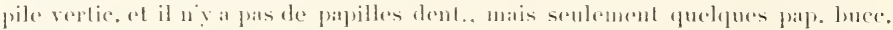

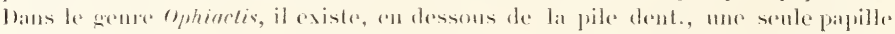

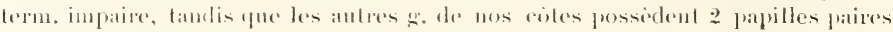
trint.

\section{(i. AMPHIURA Fin:s.}

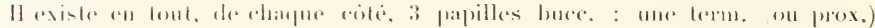

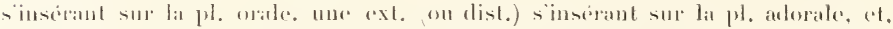

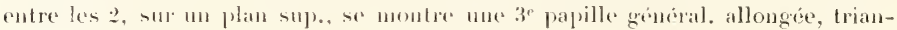
gulaire ef poinfue. Les boucliers rad, sont divergents at le dingue est depourvu de pirp. Les piq. brath. sont en nombre stpp. at 3.

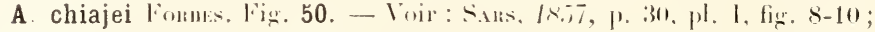

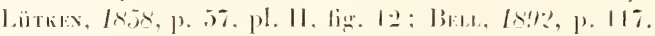

Le disque est arrondi ou pent agonal, evaré daus les espaces interrad. ; son diam. pent atteindre 10 mm. Les brats somt attomgers assez forts. el leur long. egale 7 on s fois le diam. du disque foul l'en-emble de l'animal est plutot

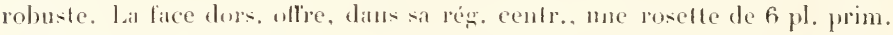
bien apparente: les boutiers rad. sout plutcil pelits et latgement divergents: la face ventr. es eomplet. comverte de pl., un peu plus petiles que sur la ders. Lat papille buec. evt. e-t elargie el rectangulaire. Les pief. brach., au

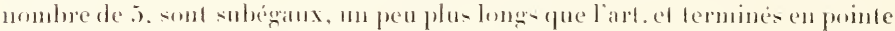
arrondie. les pl. Drach. rentr., ofleent, all moins au commencentent des bras, une saillie longit. méd. de chaque coté the litquelle sitend une dépression, ce qui leur donme un aspect camnelé. Il y a ż écailles lentac.

La couleur est rouge orangé a l'élat vivant; etle disparait dans l'alcool.

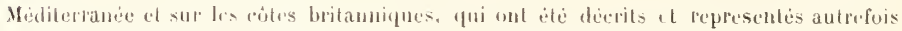
par J. MüLt.en, olfrent i paires de bras: dans la firme littorale de Roscoff, ces P'uleus sout beaucoup plus simples et ils n'ont que 2 paires de bras; entin les ceufs d'o. fragilis provenant du Pas te Calais sont gros et riches en vitellus, et ils se développent directement en ne subissant que des metamorplioses restreintes. Il serait trés important de reprondre ces études, fort interessantes d'ail'eurs au point de vue de lembrrologie générale, et de rechercher quelles relations peuvent exister entre nos liffirentes var. dophinthrir of leur mole te déchppement. 
L'A. chiajei est répandue dans toute la Néditerranée; sur nos cotes de Provence, on la trouve sourent daus les Algues it quelques m. de prof., mais elle

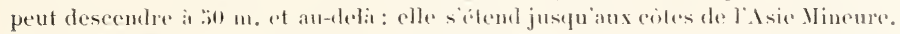

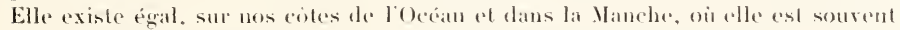
littorale. Elle se contimne sur les eotes diongletere of remonte jusquatux iles

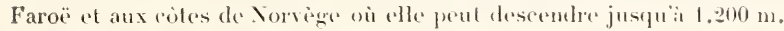

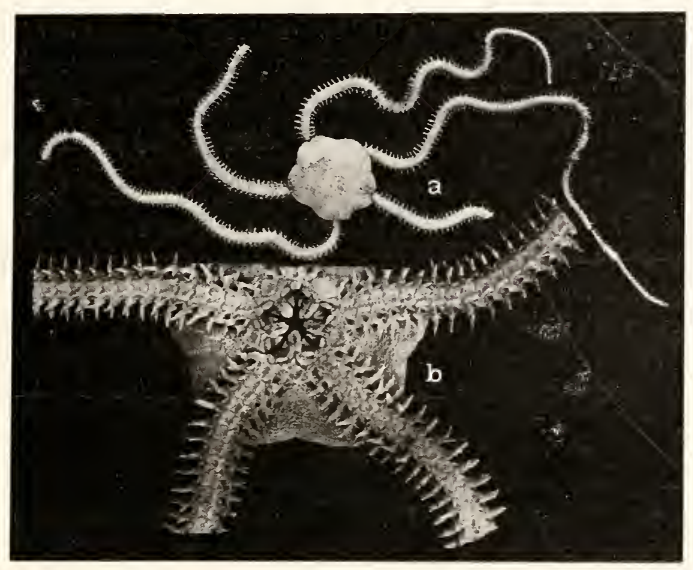

Fis. 50. - Amphiura chiajei; a. face dorsale, $X 2$; b, face ventrale, $X: \because$.

LA. chiajei se distingue lacilement de l'A. filiformis parl l'existenee de pl. sur la face ventr. du disf. el de 2 ácailles tentac. Elle se distingue de lit, medilerranea, avec laduelle elle a stó comlomblue, parl la présence d'une roselle prim. sur la face dors, du disif., par les pirf. brach. moins nombleux, par les premieres pl. brach, ventr. cannelies et par sil coloralion assez vire. On ne peut pas la confondre avec l'Amphipholis squamala qui reste loujours plus petite arec des bras plus courts portant 3 piq. seulement et dont les 3 pap. buce. se suivent en une série régul.

A. mediterranea Jrmax, Fig. 51, - Voir: L. Cиявк, 191', pl. IV, fig. 5 et 6. Cette espèce n’a pas encore été décrite; Lxwax, en 1882, sest borné à dire quelle se distingue de l'Ll. chiajei par ses piq. brach. plus nombreux; l. Clark, en a donné, en 1911, deux photographies peu démonstratives, sans la décrire.

Le disque est arrondi ou pentagonal avec les angles arrondis et les cotés quelque peu excarés; les bras sont minces, très élroils et très fins, et leur long, égale 8 à 9 fois le diam, du disque: dans un échant. comme celui de la fig. 50. le dian, du disque est de 5,i, el les bras ont f.5 mu, de longr. la face dors. 
du disque, aplatie est couverte de petites pl., nombreuses et imbriquées, subégales, et une seule pl. centr. se distingue des autres par ses dimensions plus grandes: les boucliers rad. sont petils el divergents. Ja face ventr. est couverte sur toute son étendue de pl. fris petites, plus fines que sur la lace dors. Les boucliers buce, sout losangiques, ansi latges que longs, avec un angle dist. tronqué. I.es pl. adorales, 3 fois plus longues que larges, sont rétrécies en dedanset élargies en dehors, et elles fournisient une nince lame qui sépare le bouclier buee. de la première pl. brach. Jat. : les plaques rrales sont I lois plus hautes que larges. La papille buce ext. est allongée, rectangulaire, 3 lois plus Ionğue que haute, avee le bord libre légér. excavé ; la papille intermediaire est assez minee et allongée. Les pl. brach, dors. sont très grandes, un peu plus larges que longues el triangulaires. I.es pl. brach. ventr. sout pentagonales avec un bord dist. plus ou moins fortement excavé en son milieu, et leur surface est tout i fait plane. Les piq. brach. sont au nombre de 7 ou 8 : ils sont courts, épais, arrondis, subégaux, rugueux ì l'extrém. et leur long. est égale ou inf. a celle de l'art. Les écaille- tentac. sont alu nombre de 2.

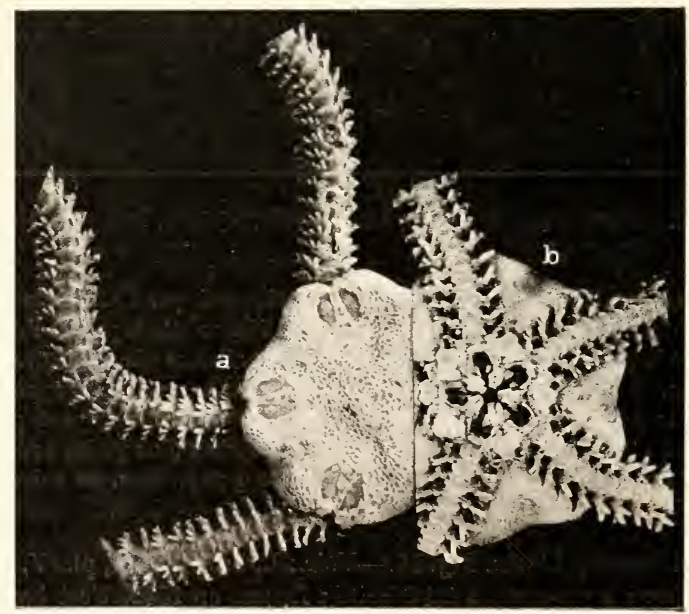

Fir. 51. - Amphiura mediterranea; a, lace dorsale, $X: 3 ;$ b, lace ventrale, $X 6$.

Lat conlen a l'elat vivant est d'un blane grisatre ne changeant pas dans l'alcool. J'ai indiqué plus haut les caractères qui séparent I'A. mediterranea

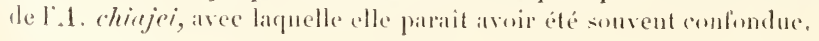




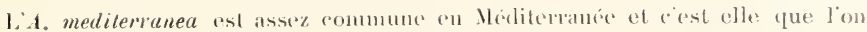

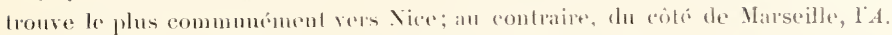

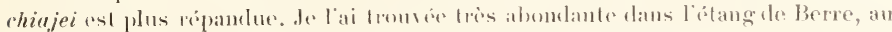

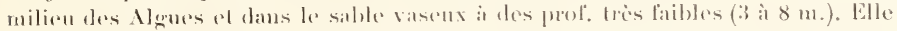
ne parait d'ailleurs jamais deserendere all delit d'une dizatue de m.

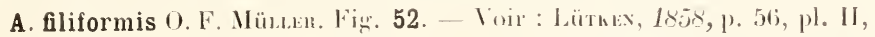
lig. 11.

1.e dian. du disque égale si i 10 mun. les bras, fris longs et tris minces peuvent alteindre 100 mm. : ils se brisent très facilement. Celle espece, qui ext fris fréquemmenl associée à 1.' chiajei, s'en distingue par l'al)sence de roselle prim. sur lis face dors. du disque, el la face ventr. en grande partie 111e: de plus il n'esiste pas d'icailles tentac, ;es 3 caractères la dislinguent à la lois de I'A. chiajei el cle l'A. mediler-

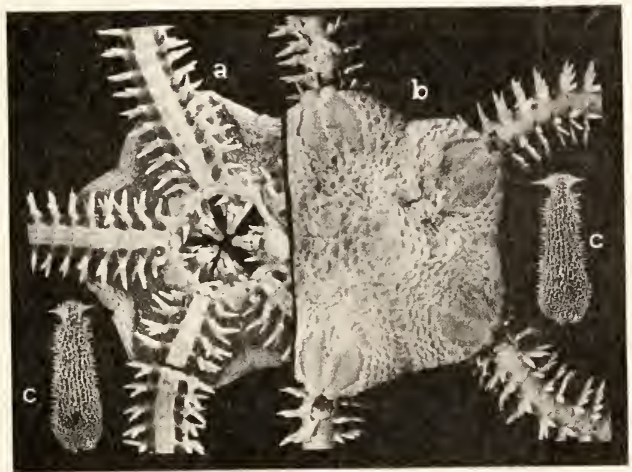
ranea. Les piq. brach. sont au nombre de 5 a 6 :

Fig. 52. - Amphiura filiformis; $\mathbf{a}$, face ventrale; $\mathbf{b}$, face dorsale, $X 6 ;$ c, deuxitme piquant ventral, $X 20 \%$. le ese piq. olfre ume structure tres caractéristique: il s"élargit an eflet à - son extrém. en ez pointes divergentes, hyalines el poinlnes faisant un angle droil aver le pin. c ; on dit que celui-ci est bihanulé.

A l'élal vivant, le disque est d'un brun rougeatre en dessous ef le bord de la face rentr. est très foncé. La liace dors. des bras est brun rongeáatre, avec sonvent un point noir et une ligne longit. runge sur les crités de chanue art. ; ces colorations disparaisisent dans l'aleool.

$\mathrm{L} A$, fliformis parait assez répandue sur nos cotes de la Manche el de l'Océan elle est notamment très commume it Dunkerque, it Wimereux, au Pouliguen; om la tronve dans le sable i mer basse, mais surtoul a la dragne, a me profondeur de 3 à : 00 m.; elle remonte jusquaux cilles de l)anemarck, de Vorvége el aux iles Faroë, et parait très ripandue daus les mers du X. Elle est égaloment assez conmune en Nóditerratue sur nos côtes dr. Provence ainsi que sur celles d'Italie;

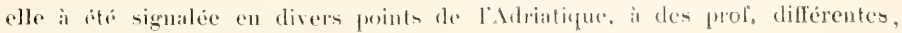
justgu 


\section{G. AMPHIPHOLIS LANGM.}

les papilles buce., au nombre de 3 , se suivent sm une meme ligne et sur un méme plan : la papille cxt. est très allongeve, rectangulaire, les 2 autres sont plus pelites, et ees 3 papilles, en rejoignant leurs congrueres, ferment complet. les fentes buce. Les bras sont courts et tres greles et les picp. brach. ordin. au nombre de 3 seulement; les boucliers rad. sont contigus. Ce g. ne renferme que des especes très petites ot délicates.

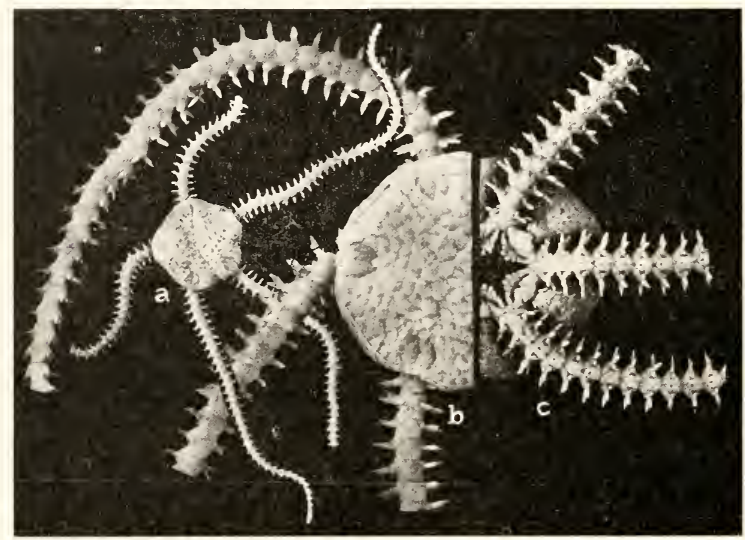

Fig. 53. - Amphipholis squamata; a, face dorsale, $X 4 ; \mathrm{b}$, face dorsale. et c, face ventrale, $\times 12$.

A. squamata Delle Cinsu: Amphiura s, auct., A. elegans Leach, A. neglecta Joxstox . Fig. 53. - Voir : Bess, ls.?, p. 119 [Amphiura elegans].

l.e diant. du disque varie entre 3 et $3,5 \mathrm{~mm}$. et atteint rarement $4 \mathrm{~mm}$. : les bras ne dépassent guère 12 a l.j mum. : ils sont trés greles et cassants. Ia

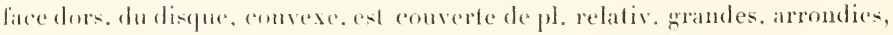

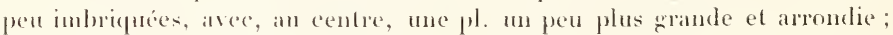
les pl. ventr., petites et imbriquées, forment un revètement continu. Les beailles tentac, au nombre de 2 , sont presque ég.

La couleur à l'état viv. est d’un gris unif., plus ou moins foncé.

L'A. squamata est extrêm. commun sur toutes nos còtes où elle vit parmi les Algues, sous les pierres, contre les jetées des ports, atc. ; elle est surtout littorale main pent dencendre it $130 \mathrm{~m}$. ef plus. Ciest une esprece prespue cosmopolite. 


\section{(i. PARAMPHIURA KiHLEK。}

Voir : Komlen, $1895, \mathrm{p} .17$, pl. IX, tig. 22 et 23.

Amphiuriclée dont le disque est couvert sur ses faces de petites pl. inbriquées sans inclication de pl. prim., les boucliers rad. sont allongés et très étroits. La papille bucc. ext. prend un développement considérable : elle dépasse beaueoup la taille des pl. orales; de plus elle est presque accolée à sa congénère sur la ligne interrad. méd. Deux écailles tentac. Les piq. brach., en nombre sup. à 3 et subeginux, restent appliqués sur les cotes des bras.

P. punctata Fониes). Fig. 5 t. - Le disque est pentagonal, assez fortement excavé dans les espaces interrad.; son diam. est de 3 mm. seulement et les bras ont $15 \mathrm{~mm}$. de long. l.es boucliers rad., trèstins, se terminent en dedans par une pointe tries aiguë : ils restent paralleles l'un à l'autre et séparés sur toute leur long. Les piq. brach. sont au nombre de 5, subégaux, et leur long. égale à peu près celle de l'art.; leur extrin. arrondie est un peu rugueuse.

La $P$. punctata n'est connue jusqü $" \mathrm{a}$ présent que par 2 exempl. : l'un est le type de Fонвеs et il avait été trouvé dans l'estomac d'une Morue, l'autre est celui que jai décrit en 1893; il mavait été donué. par Giand qui l'avait drague dans le Pas-de-Calais sans antre indieation).

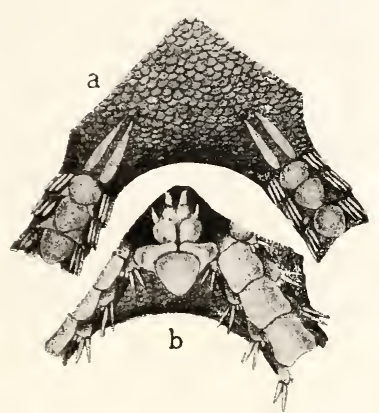

Fig. 54. - Paraniphiura punctata, $a$, face dorsale; $b$, face ventrale, $\times 12$.

\section{G. OPHIAGTIS Lütkex.}

Amphiuridée nc possédant qu'une papille buce. termin. impaire, et 1 ou 2 papilles lat. Les esp. sont en général d'assez petite taille avec des bras de moyenne long., munis de piq. un peu forts; les pl. du disque sont armées de piq. qui restent parfois localisés sur les berds de celui-ci.

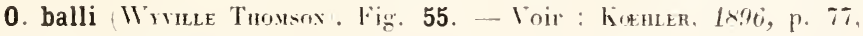
pl. III, fig. 23 et 2 í.

Le diam. du disque ne dépasse guère $4.5 \mathrm{~mm}$. et les bras ont environ $20 \mathrm{~mm}$. de long.; la face dors. du disque est converte de petites pl. imbriquées, à peu près égales, sauf une pl. centr. plus grande ; les boucliers rad. sont petits et divergents. Les piq. se montrent vers la périphérie du disque et ils se contimuent sur la face veutr. I.es boucliers bucc. sout un 
peu trilohés, à peu près aussi larges que long, il n’existe qu'une papille buec. lat. Les piq. brach., au

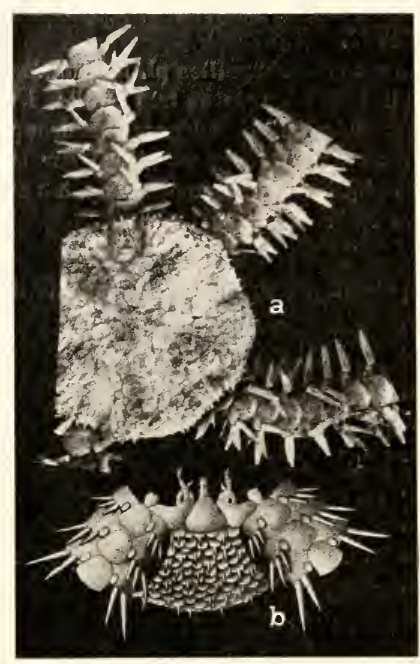

Fin. 55. - Ophentetis balli; a, face torsale, $X \times ;$ b. liee ventrale, $X 12$. nombre de 4 à 5 , sont un peu plus mands que liart., spinuleux i l'extrem. L'eratle tentac. mique, e-t mrancle el walaire.

Lat couleur a l'étal vivant en d d"un brun elair, un peu rougealre: les bras oflrent parfois des anmulations phas fonceses. Ia coloralion es en partice ennservee dans laleowl.

I.1). belli niest eneere enomue que datum I Itantipue of elle se lroure stur motre platean eontinental, toujour a une eretaine prot. ; on peut la dragner

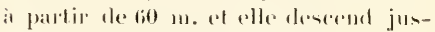

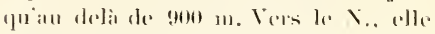
remonte justuans iles faroë of aus cribes de Norrege, mais elle nest pas eomnue en dessous du $40^{\circ}$ latit. $N$.

*0. virens SARs Fig. 56. - Vin': siмвотн, $1 \times 76$ et $1 \times 77,1$. 117 al 11!); Kindl:k, 191\%, p. 185., pl. I. lig. í el i.

Celte espece, loujours tris pretitc. $\therefore$ distiogue immediatement par te nombre de ser bras qui est constamment de 6 a a l'elat adulte. Le diam. du disque ue depasse gaère $3,5 \mathrm{~mm}$, ; les bras ont 15 a $18 \mathrm{~mm}$. de long.

Le disque est arrondi, un peu proéminent dans les espaces interrad.; sa fice dors. convese est couverte de petiles pl. irrégul. arrondies, peu on pas imbriqués, incegales, sans la moindre indication de pl. prim. el devenant plus petites vers la périphérie où quelques-unes sont armées d'un petit piq. eonique el tres contr: les boucliers rad., tres petits, sont plus on moins

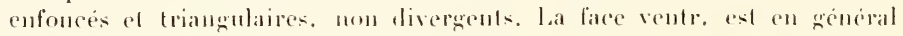

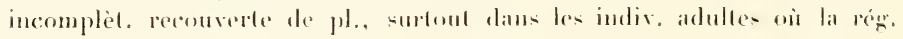
prosim. du disq. reste nue: ress la perijuherie de celui-ci, il existe quelques pelits piq. courls, coniques, identiques à ceux de la face dors. la papille termin. impaire qui se trouve en dessons de la pile dent. est 2 fois plus Inngue que large. I.es pl. brach. dors., tres larges, couvreut presque toute la face dors des luas; les pl, venlr. sont pentagrouales, lécrèr. plus larges que longues. I.es piq. brach., au nombre de 4 , sout petits, cylindriques, arrondis

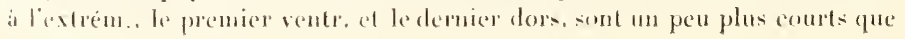


les 2 movens dont la longr. égrale celle de l'art. I'ne seule écaille tenlac., graude ef ovaliaire.

A l'atal vivant, la face dors. du disque offre une coloration d'un gris jaunâtre ou verditre avee des taches plus foncées; les bras sont plus on moins neltement annelés; la face ventr. est très claire. Ces colorations sont en partie conserves daus l'aleool.

LO virens est très remarquable par les phenomènes de reproduction asexuelle quielle présente : son disque peut se partager en deux moitiés à peu près ígales, portant chacune trois brats

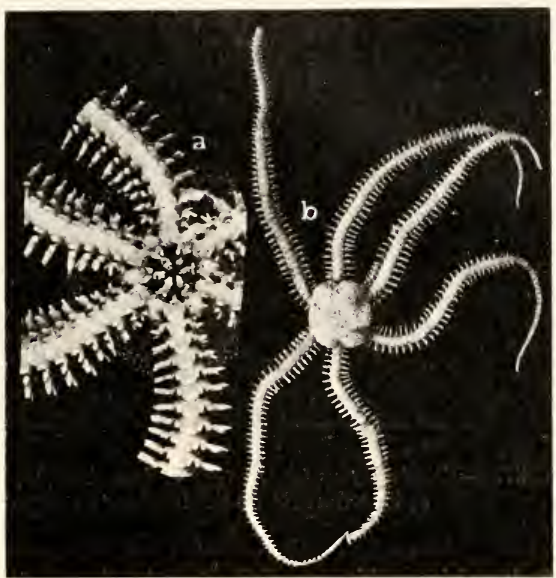

Fit: 56. - Ophinctis rirens; a, face ventrale, $\ 10$ : b, face dorsale, $X$ i. of qui rógrinement resperlivement les trois autres bras ansi que l'aulse moitio du disque : il existe des dispositions antitomiques spéciales, en rapport aree ees phénomènes de division, quion trouvera résumés daus le travail de Cínot, 1891 p. pis.

LOO. virens n'a encore été signalée jusqu ici qu’à Naples et à Madère, mais elle exsste certainement dans des localités intermédiaires entre ces 2 rég. sur les eôtes N. ou S. de la Méditerrannée, et on la trouvera très vaisemblablement un jour sur nos cotes de Provence ou d'Agérie; jai done cru devoir la mentionner ici. Elle se distingue facilement de l'O. balli par le nombre des bras, par sa coloration, par les boucliers rad. non divergents, etc.

\section{G. OPHIOCENTRUS Lavgan Amphiocnida Virmit.}

Les papilles buec. sont disposées comme dans le g. Amphiura, ed d. qu il existe de chaque côté une papille termin. insérée sur la pl, orale, une papille dist. ou ext. insérée sur la pl, adorale, et, enfin, sur un plan supérieur, une papille intermédiaire triangulaire et pointue. Le disque porte de petits piq.; les écailles tentac. font défaut ou n’existent quà la base des bras, ce qui arrive précisément dans l'espèce française où les bras sont particul. longs et portent de nombreux piq. 


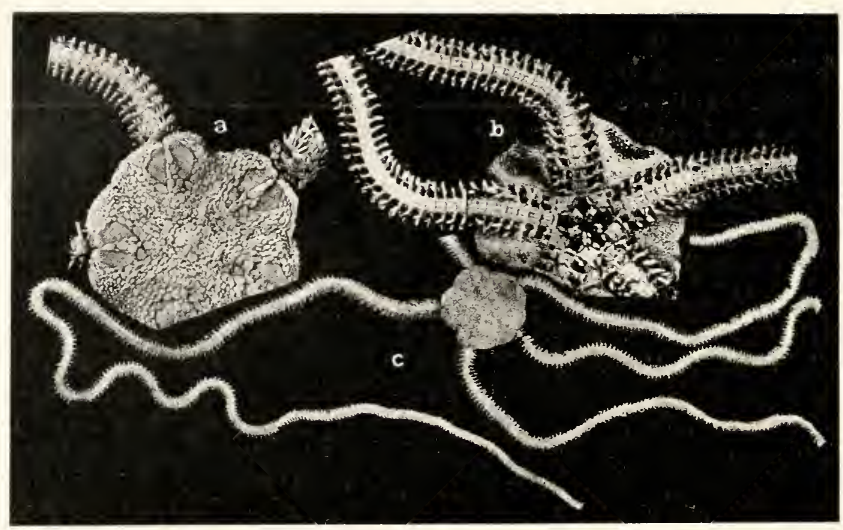

Fin. 57. - Ophiocentmus brachiatus; a, face dorsale, $X_{4}^{4} ; \mathbf{b}$, face ventrale, $X_{4}$; c, face dorsale de l'animal entier légèrement grossie.

0. brachiatus Moxrtgr Ophiocnida br. auct.]. Fig. 57. - Voir : Bell, 189.2, p. 116, pl. XIII, fig. 3-5 Ophiocnida.

Le diam. du disque atteint 8 a 111 mm. ; les bras sont extrêm. longs et le dépassent 15 à 20 fois ce diam, ; ils sout aplatis et assez minces. La face dors. du disque offre une rosette de $6 \mathrm{pl}$. prim. un peu plus grandes que les voisines qui sont nombreuses et un peu imbriquées: vers la périphérie, elles deviennent plus petites en même temps qu'elles s'épaississent, se redressent et acquièrent un petit piq. court, conique et pointu; les boucliers rad. sont assez grands et peu divergents. La face ventr. est couverte de pl. épaisses, dressées, et portant aussi chacune un petit piquant. Les boucliers bucc. sont presque losangiques: les pl. adorales sont rétrécies, mais contiguës en dedans. La papille bucc. ext. est tres grande et squamiforme. Les pl. brach. dors. sont grandes ; les ventr.. quadrangulaires, offrent à leur surf. 3 saillies longit., une méd. et deux lat., séparées par 22 sillons ou cannelures qui s'étendent jusqu’à une certaine distance du disque. Les piq. brach. sont au nombre de 7 ì 9, parfois même de 10, un peu plus petits que l'art., aplatis, avec l'extrém. arrondie. Jes pores leutac. offrent sur les premiers art. 2 écailles ; au delì du disque, l'écaille int. disparait et l'ext. persiste seule en se réduizant progressivement. puis clle ne larde pas à disparâ̂tre à son lour el les pores restent nus.

La couleur a l'utat vivant est d'un grris rougeâtre ou jaunâtre assez terne et miforme qui passe complit. dans lalcool.

Sur nos eôtes de l'Mllantique, l'(), brachiatus vit dans les sables vaseux à 10 ou 
$20 \mathrm{em}$. de prof. ; ses bras démesurément longs, sont très souples et peu cassants; à Areachon, où elle est assez eonmune, elle se trouve associce it tés Solen, it la Leptosynapla digitata, ete.; elle a été assez rarement signalée, mais doit être fréquente sur nos plages de sable. Elle remonte sur les côtes des lles Britanniques, mais ne dépasse paś le $56^{\circ}$ latit. N. En Néditerranér, l’o. brachinlus a ćté rencontrée à Marseille el à Naples, dans les sables vaseux, it des prof. toujours faibles et ne lépassant pas $30 \mathrm{~m}$.

On reconnait facilement eette espeece à ses bras extrêm. Iongs et à sou disque armé de piq.

\section{S. CL. CHILOPHIURIDES}

\section{F. OPHIODERMATIDÆ LJUNGMAN.}

Le tisque est muni sur les 2 faces de granules arrondis et serrés, reeourrant on non les boueliers rad. et les pl. adorales ainsi que portés par des pl. extrên. minees et imbriquées. Il existe des dents et de nombrenses papilles buee. mais pas de papilles dent.; les bras, eylindriques et flexibles, portent de nombreux piq. courts, ordin. appliqués contre les pl. lat.

\section{G. OPHIOderma Mülier et Troschel.}

Les dents sout coniques et pointues. Les piq. lrach., atteignent le elitlre de 10 on 12 ; ils sont très courts, aplatis, plus pelits que l'art., très étroits, appliqués et formant une série ininterrompue. Les ieailles tentae. sont au nombre de 2, l'ext. recouvrant la base du premier piq. brach. La face dors. du disque offre, à la base des bras, une incisure profonde, dans laquelle sont reçues les premières pl. brach. dors. Ia fente génit. au lien d'ètre unique le long de chaque bras, est dédoublée : il existe une fente proxim. vers le bouclier bnec. et une autre dist. vers le bord du disque. Les indiv, atteignent général. une grande taille.

0. longicauda Lixck. Fig. 58. - Voir : Mïller el Troscnel. 18'?, p. 86, pl. IX, fig. 1 ; Koenler, I!'/', p. 173, pl. IY, fig. 1-7.

Le diam. du disque alteint 25 mm., el les bras oul 100 à $150 \mathrm{~mm}$. de long.; leur larg., à la base, varie entre 1 et $\mathbf{1}, 5$ mm. : lout l'ensemble de l'animal est robuste el ses mourements sont très vifs.

Les 2 fices du disque sont couvertes de granules frès lins, sphériques et serrés, qui, sur certains exempl., recouvrent les boucliers rad., et sur 
d'autres les laissent à nu; ces granules recourrent égal. les pièces bucc. les pl. brach, dor's. sont très grandes, rectangulaires et ordin. morcelées en 2 fragments par un sillon méd., auquel s'ajoute parfois 1 ou 2 autres sillons

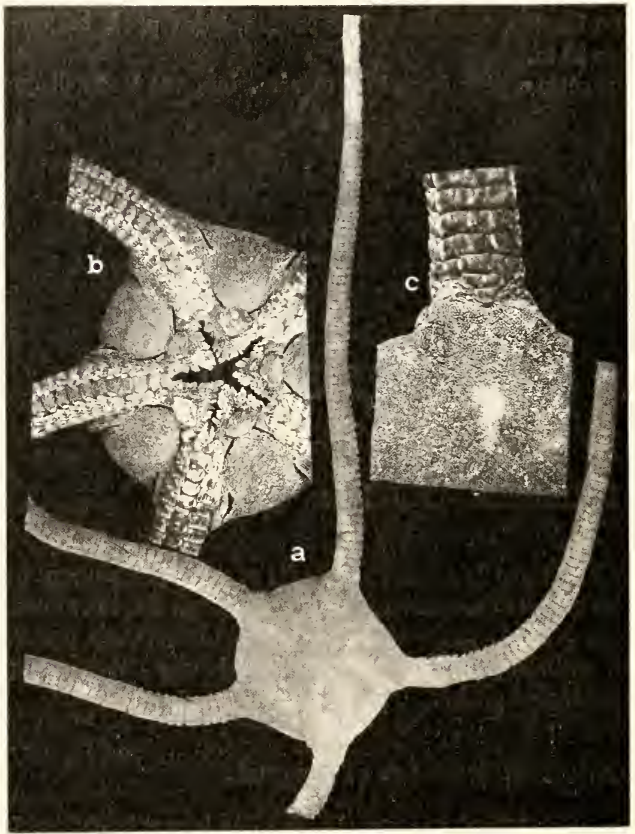

Fin. 58. - Ophioderma longicauda; a, face dorsale Jégèrement l'éduite: b, face voutrale lígìnement chrossie: c, face dorsale à l'orinine d'un luras, $X 2$. lat. : mais elles peuvent égal. resfer entières el l'on olserve beaucoup de variations a cet égard: le plus souvent il y a alternance entre une série de pl. morcelées ell 2, 3 ou i fragments, et une série de pl. entieres. Les piq. brach. dépassent un peu la moilié de l'art., et le premier piq. ventr. est un pen plus longqueles autres, q,uj sont $10 u$ s égaux.

4 l'etatrivant, la coloration est très foncée, d’un brun noirâtre sur la face dors.; la face ventr. est beaucoup plus claire, grise ou jaune grisâtre. Tantôt la coloration est uni-

\section{coloration est min-}

forme, timtot il existe quelques petites laches claire sur le disque; les brus peuvent aussi olfrir d'issom grandes tarches grises allongées transvers. el très inégal. distribuces. La coloration se conserve dans l'alcool.

L'O. longicauda est surtout répandue en lléditerranie où elle est très fréquente sur tout notre littoral ; on la trouve sur les rochers batlus par la mer, an milieu des Algues, dans les fentes des pierves, contre les jetios des ports, où on la voit sagiter à une faible prof., vers 0,00 cus., ot on peut la capturer facilement à l'aide d'un hamegon muni d'une amoree animale; de pent d'ailleurs descendre

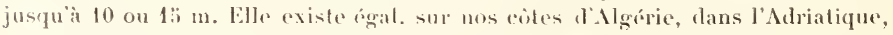
ete. Dans l'Atlantique, elle deseend lo loug des cotes d'Espagne, du Portugat et 
d'Afrique jusqu'a l'ḱquateur. et elle est tròs commune aux Acores et it Marlire: sur nos côtes occidentales, elle ne paraît pas dépasser La Rochelle vers le $\mathrm{N}$.

\section{G. OPHIOCONIS L̈̈TKPN.}

l.es gramules reeouvent les boneliers rad. ef toutes les pl. huce. Les dents sont aplaties ot très minces, en forme de lanelles translucides aver quelques denticulations sur les bords. Les papilles buce. lat. sont nombreuses, la plus ext. est la plus petite. Les piq. brach. sont courts, aplatis, hyalins, plus ou moins dressés; les pl. brach, ventr, sont très allongées. La taille reste petile.

0. forbesi Hellek . Vig. 59, - Voir : IILLen, 1863, p. \{2.2. pl. II, fig. 5-2 Pectinura $f$. .

Le diam. du disque est de 5 à 7 mm. les brascunont gucure plus de 15 à $18 \mathrm{~mm}$. de long. et égalent à peu près 3 ou 3 fois 1 2 le diam. du disque. Celui-ci est unilormément comvert degramulesqui, sur les pl. adorales et orales deviennent un peu plus grossiers surtout au voisinage de la pile dentaire. Les pl. brach. dors. sont grandes et translueides, plus longues que larges; les

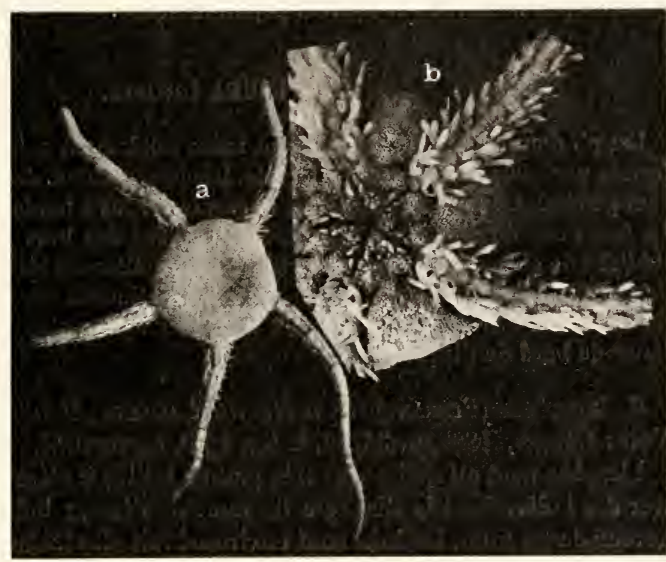

Fu. 59. - Ophioconis forbesi; a, face dorsale, $X i$ : b, face ventrale, $\times 10$. pl. brach. ven-

1r. sont aussi plus lomgues que larges. Les piq. brach. sont an uombre de 7 ; les premiers piq. ventr. et les derniers dors. égalent l'art. el les moyens sont un peu plus courts. Les pl. brach. présentent des stries transv. très fines el parallèles. L'écaille tentac. est très grande, ovalaire ou lancéolée, beancoup plus longue que large; et il en existe 2 sur les premiers art. brach.

La couleur à l'élat vivant est jaune Immâtre avee des marbrures plus foncées; les indiv. en aleool sont blanes.

Cette espèce a été considérée pendant longtemps comme spéciale à la Méditer- 
ranée; on la trouve au large de nos côtes de Provence, dans les graviers et les sables des "fonds durs" de la "broundo ", au inilieu des Algues calcaires, vers 40 à $50 \mathrm{~m}$. de prof., associée à l'Ophiura albida qui est toujours plus grande qu'elle: elle n'est pas commune. On l'a signaléc à Messine, à lissa et à Corfou, entre 20 et $60 \mathrm{~m}$. Elle a été rencontrée aussi dans les parages des Açores, entre 90 et $208 \mathrm{~m}$.

\section{F. OPHIOLEPIDIDÆ LuUngmaN.}

Le dirque est couvert de pl. général. grandes, inégales et épaisnes. Il n'y a pas de papilles dent., mais seulement des papilles buce.; les dents forment une rangée vertic. Les bras sont relativ. forts, rigides et résistants, larges à la base et allant en se rétrécissant rapidement. Les pir. brach, sont petits, peu importants. général. peu nombreux et souvent papilliformes.

\section{G. OPHIURA LAMARCK.}

Les pl. dors. du disque, fortes, sont unies solidement entre elles, de manière à former une sorte de carapace résistante. les pores tentac. des premiers art. brach. sont général. très grands, ovalaires et garnis sur les 2 bords de plusieurs écailles. La face dors. du disque est échancrée à la base des bras et les incisures sont limitées de chaque côtẻ par une rangée de papilles serrées, formant une sorte de peigne, le peigne radial; ces papilles se continuent sur la face ventr. le long du borl ext. des fentes génit.: les boucliers rad. restent à une certaine distance du bord lu disque.

0. lacertosa (Pexint) [O. texturata Lamarck, O. ciliaris L.]. Fig. 60. Voir: Lütín, 1858, p. 36, pl. 1, fig. 1 [O. texturata].

L'espèce peut atteindre une très grande taille : il n'est pas rare de rencontrer des indiv. dont le disque a $35 \mathrm{~mm}$. de diam.; habit. ce diam. oscille autour de $25 \mathrm{~mm}$., les bras sont environ f fois plus longs. Ce disque est épais et arrondi; sa surf. dors, est convexe ot la face ventr. plane. Les bras sont larges a leur base qui mesure 5 mm. : leur face dors, se relève en une carène arrondie; la larg. diminue rapirlement jusqu’̀ l'extrém. qui est très amincie et pointue. Ces bras sont tout i fait rigides et ils semblent ne ponvoir elfectuer que des mouvements peu étendus: lont l'ensemble de l'animal est très robuste.

La face dors. du disque est couverte de pl. très inćgales, général. assez petiles, parmi lesquelles on remarque une c.-dors. arrondie, el, séparées d'elle par 1 ou 2 rangs de pl., 5 pl. rad. prim. Les papillen rad. sont d'abord extrèm. fones et allongées, puis elles sélargissent progressiv., en mêne temps qu'elles se raceourcissent pour passer sur la face ventr. on pent compter une trentaine de papilles sur la face dors. Les incisures rad. sont grandes et profondes et elles reçoivent les 5 premières pl. brach. dors. Celles-ci portent 
sur leur bord ext. chacune quelques papilles extrêm. petites, beaweoup plus petites que celles du peigne principal, en dessous duquel elles forment une sorte de petit peigne aceessoire. Les boucliers buce. sont très grands, lancéolés, plus longs que larges, feur long. dépassant beaucoup l'espace qui les sépare du bord du disque. Les premières pl, brach, ventr, sont séparées les unes des autres par une petite dépression élargie fransvers. : ees dépressions sont très marquées sur les art. situés en dedans du disq.; les í ou jsuivantes s’alténuent progress, puis elles disparaisent finalement, Les piy.

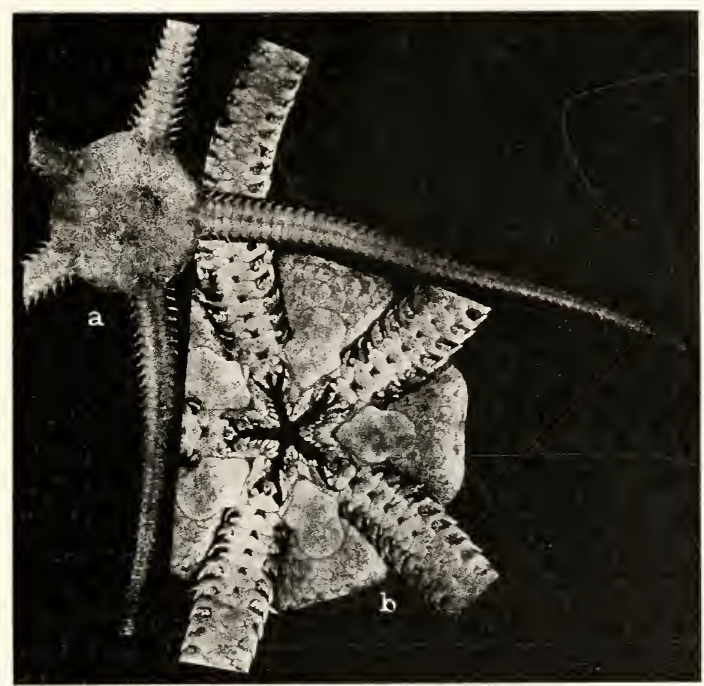

Fuc. 60. - Ophiura larertosa: a, fare dorsale lógèrement réduite: b. face ventrale, $X:, ;$.

brach.. au nombre de 3. sont très réduits. Les prẹmiers pores tentac. sont très grands et limités par 1 ou jóeailles de chaque coté, puis ce nombre diminue, tombe a 2 ou 3 , puis finalement il ne reste quime seule écaille dans la partie termin. des bras.

La couleur à l'état vivant est orangée ou rougeâtre sur la l'ace dors., plus pâle sur la faee ventr. : elle disparait eomplèt, dans l'aleool.

L'o. lacertosa est répaudue sur toutes nos côtes et vit surtout dans les fonds vaseux et sableux. Dans lAtlantique, on peut la trouver i mer basse sur certaine plages sableuses (Arcachon), mais elle vit surtout it partir de quelques mo de 
prof. et peut descendre jusqu'i $200 \mathrm{~m}$. ; elle s'étend jusqu'aux côtes de Norvège. En Méditerranée, l'O. lacertosa est très commune, principal. dans les fonds vaseux à partir d'une dizaine de m. ; clle est très répandue sur le pourtour de la "broundo " et au delà dans fous les sables vasenx ou la vase du large où elle descemal jusqü ì $300 \mathrm{~m}$.

0. albida lonms. Fign. 61, - Voir : Lётиғ, 1858, p. 39, pl. 1, fig. 2.

L'O. albida est très

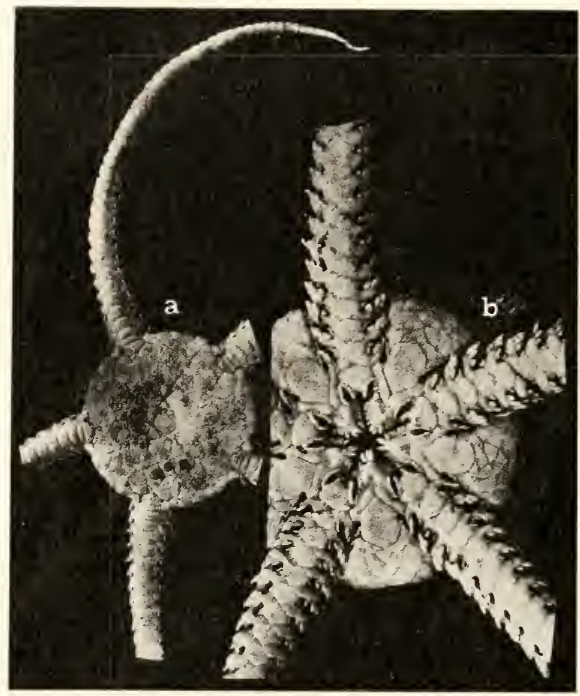

Firi, 61. - Ophiura albida; a, face dorsale, $X 2:$ b. face ventrale, $X 4$. voisine de l'O.lacertosa, mais elle est toujours plus petite; le diam. du disque ne dépasse guère 10) ¿ $12 \mathrm{mmm}$. et la long. des bras atteint environ 1 fois ce diam. ; elle est assez robuste, ses bras sont rigides et le revêtement du disque est très solide. La face dors. du disque est couverte de pl. inégales, assez grandes, offrant une rosette prim. de 6 grandes pl. arrondies et contiguës. Les incisures rad. sont moins prof. que chez l'O. lacertosa, et ne contiennent que les 3 premières pl. brach. dors. Les papilles rad. sont basses, assez épaisses et subégales: on n'en aperçoit que 15 à 18 environ quand on regarde lophiure par en haut. Il existe aussi mu petit peigne rad. supplémentaire. Les boucliers buce. ne sont pastrès grands, plus longs que larges et pentagonaux aveo les côtés droits; leur long. est égale ou inf. à l'espace qui les sépare du bord du disque. Il n’y a pas la moindre trace de dépressions entre les premières pl. brach, ventr. Les piq. brach, au nombre de 3, sont petils.

I l'ibil vivaut, la lace dors. du disque el des bras ost jaune orangé avec des marbrures blanches: la face ventr. est plus clatre: la coloralion dispatrait complèt. dans lableool.

l'̈l. albida se rencontre dans les mêmes localités que l’o. lacertosa, mais elle

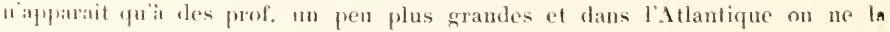


trouve pas à mer basse. En Méditerrance, elle se montre à partir d'une dizatine de m. Elle parail deseentre à des prof. plus grandes que l’O. lacertosa (833) 11. dans le eanal des ile's lanoë).

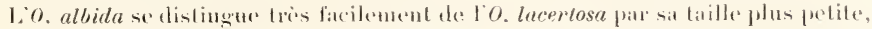
par les ple dors. du disegue relativ. plus grandes, par l'absenere de depressions entre les premieres pl. brach. ventre, pitr les patpilles pald plus courtes el moins nombreuses, par les bouclier's buec, plus couth, elc.

\section{F. OPHIOCOMIDA LJUNGMAN.}

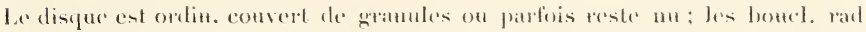
sont visibles. 11 existe des papilles dent. qui forment un paquel rertic, en dessous

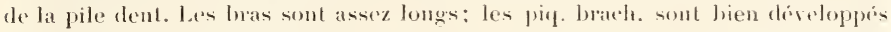
et dressés; 1 ou 2 écailles lentac. Celte fanille eomprend deux sons-lamilles :

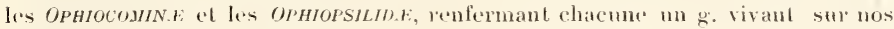
coites.

\section{G. OPHIOCOMINA KinLEH.}

Voir : Kinten, 1921 b; pl. L.XXY, lig. 1-6 (sous presse).

Ophiocomine chez laquelle les pic. brach. sont creux comme dans le genre "phiacantha. Le disfue est couvert de gratules sur les 2 faces. les boucliers buec. sont darquis transvers, fes pl. adlorales, très allongeres, sont contigues sur la ligne interrad. midl., elles s'alargissent en dehor's et separent plus ou moins largement le bouclier buer. de lat fremière pl. brach. lat. Les pl. orales sont hautes; les papilles buce, el dent. sont disposies comme dans le g. "ophiocoma, c. à d. que les papilles buec. sont assez nombreuses et les pap. dent. forment un paquet assez serré. 2 écailles tentac.

Ce g. est représenté par une seule espèce qui vit dans l'Atlantique boréal, et peut pénétrer en Méditerranée. Jai disculé les caractères du g. Ophiocomina et Jes raisons pour lesquelles j’ai cru devoir le séparer du g. Ophiocoma, dans le travail signalé ei-dessus, auquel je renvoie le lecteur.

0. nigra (O. F. MüLlzk). Fig. 62. - Le disque est arrondi el assez épais ; son diam. varie ordin. entre 12 el 15 mm., mais il pent arriver jusqu'a 23 mon.; la long. des bras atleint is un lo lois ce chillre. La face dors. est miformément couverte re gramules arrondis et serrés, qui cachent complèt. les pl. sousjacentes et les boucliers rad.; le disque forme, sur sa face dors., a la basc des bras, une légère incisure dans laquelle sunt reçues les ¿- premières pl. brach, dors. plus petites que les suivantes; la face ventr. est aussi couverte de granules. Les piq. brach., au nombre de 6, sont cylindriques, asse\% minces, avec l'extrém. arrondie et finement denticulée sur toute leur long. 
La face dors. du disque est très linncée, d'un brum noiratre, les bras sont plus clairs ef bruns, la face ventr. est plus claire également. Cette coloration se conserve dans lalcool. Il existe aussi, dans les mers du X., certains indiv. dont la couleur ent orangée ou mème rome, el qui se décolorent dans lalconl.

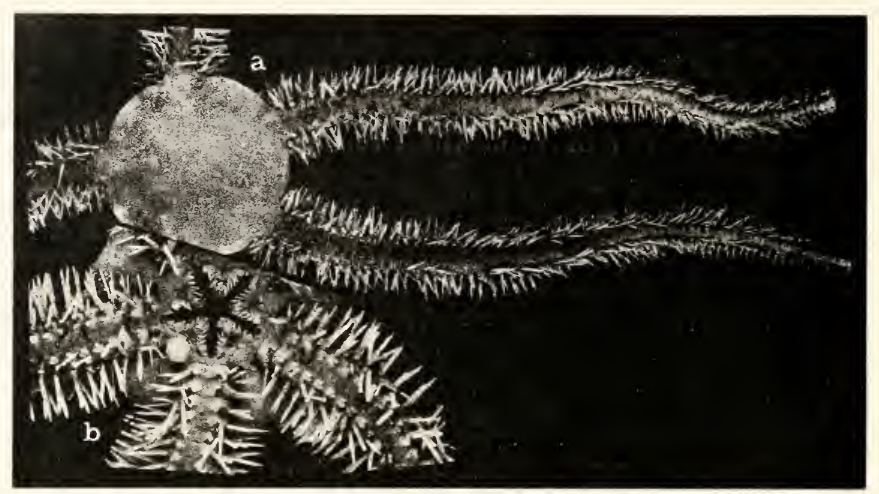

Fig. 62. - Ophiocomina nigra; a, face dorsale, $X 2$; fice ventrale, $\mathbf{b}, \times 3$.

L'O. nigra vit surtout dans l'Atlantique; elle est assez répandue sur nos cótes, surtout à Roscoff et à Concarneau, dans des sables graveleux et des fonds rocailleux, entre 6 et $30 \mathrm{~m}$. de prof., mais on ne la trouve pas it mer basse. Elle remonte assez haut dans les mer's du X., sur les cotes des Iles Britanniques et de Torvège, oì on peut la trouver à mer basse dans la zone des Laminaires : d'autre part, elle descend jusquà $160 \mathrm{~m}$. Comme elle a été rencontrée aux $\Lambda$ çores, il est Irès vraisemblable quelle se trouvera sur nos cotes du S. W. Enfin il est certain que l'o. nigra existe en Méditerranée; jen possède un échant. provenant de sicile,

\section{G. OPHIOPSILA FoHBKS.}

1. disque est comert, sur so 2 faces, de pl. extrim. minces ot pelites, difficiles i aperceroir: les boucliers rad, sont tres allongés et itroits. Il existe is la fois des papilles buee, et des papilles dent. Les piq. brach. sont courts, assez nombreux et dressés. Le caractere essentiel du g. est offert par les écailles tentac. dont l'int. se prolonge en forme d'un long piq., aplati et lancéolé, qui se dirige obliqu. sous la pl. brach. ventr. correspondante et se croise avec son congénère, tandis que l'ecaille ext. reste petite et courte. 


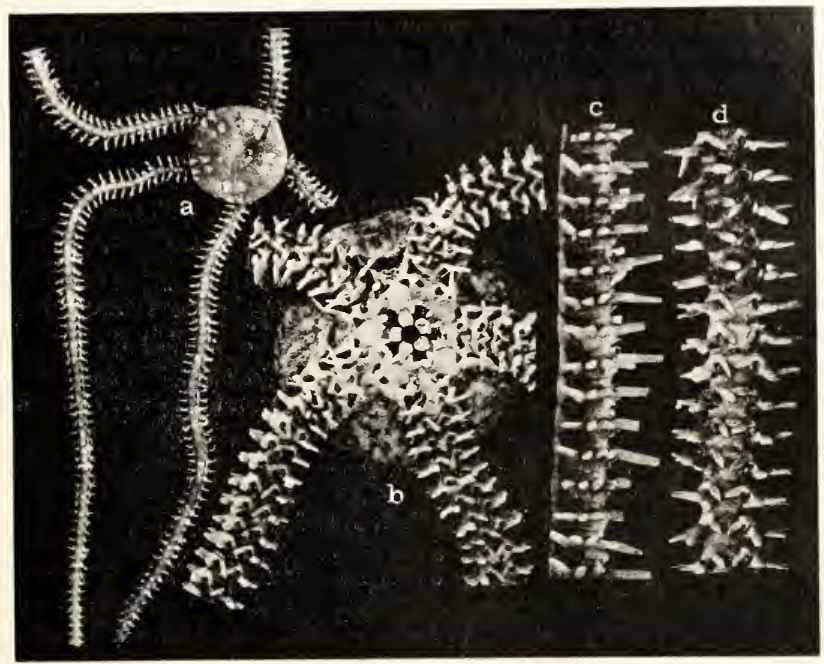

Fis. 63. - Ophiopsila aranea; a. faee dorsale, $X 2$; b, faee rentrale et c, vue latérale d'un bras, $X i$; $d$, faee ventrale d'un bras, $X 7$.

0. aranea Fokвes. Fig. 63. Voir : Fonвts 18'19, p. 149, pl. XIV, fig. 1-7; Heller, 1863, p. 415 , pl. II, fig. 17-20: Kuenlek, 1914, p. 205, pl. V'III, fig. 5 et 9 .

Le disque est petil, arrondi et son diam. ne dépasse pas 6 à $7 \mathrm{~mm}$.; les bras sont très étroits, délicats, leur long. varie entre to et $15 \mathrm{~mm}$. ; chez l'animal vivant, ces bras se meuvent avec agilité en se contournant et ils se brisent facilenent. Tout l'ensemble de l'Ophiure est délicat. La face dors. du disque est couverte d'un tég. mou et qui parait dépourvu de plaques, mais un examen attentif sur des échant. desséchés permet de découvrir de très petites pl. imbriquées el arrondies, qui laissent à nu les boucliers rad. ; ceux-ci sont étroits, très allongès, largement séparés l'un de l'autre. Les piq. hrach., au nombre de 6 , sont aplatis, arrondis à l'extrém., subégaux, le premier ventr. un peu plus grand que les autres, et le dernier dors. un peu plus petil. L'écaille tentac. int, allongée, est fusiforme et assez large; l'écaille ext. est petite et pointue.

La coul. à l'état vivant est d'un brun rougeâtre, avec, sur la face dors. du disque, des taches blanches irrég. qu'on peut retrouver sur les bras; la face ventr. est plus claire et jaunâtre; ces colorations se conservent plus ou moins dius l'alcool. 
$\mathrm{L}^{\circ} O$. aranea est très répandue en Héditerranie oil elle vit général, entre 30 et $50 \mathrm{ku}$. de prof. dans les graviers et surtout parmi les algnes caleaires : elle a été signalfie sur nos cotes de Provence et sur les eotes dAlgérie, a Naples, dans l'Adriatique, dans la mer Égée, ete. Elle existe anssi dans la Manche et Pmyor l'indique à Roneofl dans les graviers littoraux et le sable colier lin. Enfin, on l'a trousce aux Acores (91)-18:" nu.).

"0. annulosa Sirs. Fig. 64. - Voir: SArs, IN.7, 1, 79, pl. I, tig. 2-7 ('phianoplus : Kinsms, I9/'ı, p. 205, pl. VIII, fig. 6 ef 12.

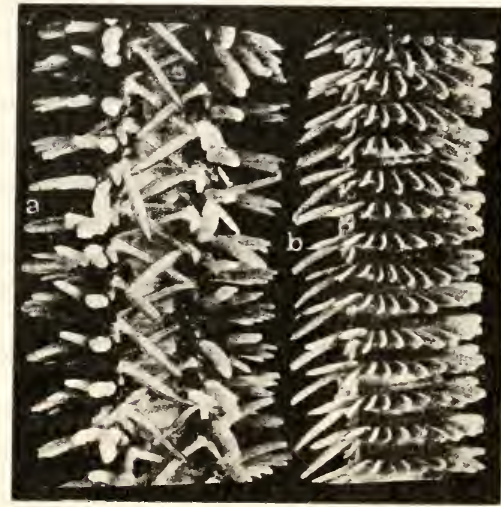

Fin, 64. - Ophiopsila annulosa; a, lare ventrale d'un bras et $b$, ve latérale d'un bras, $X 6$.

Cette espece se distingue de la précédente par sa grande taille et sa structure très robuste : le diam. du disrpue atteint facil. 10 a 120 $\mathbf{2 m}$., el la longueur des bras alteint au moins douze fois ce chiffre: ces bras sonl arroudis, aplatis sur la face ventr. el assey gros. l.e disque porte sur ses ? laces des écailles tress lines ef tres minces, un pen plus developpées au voisinage des boncliers rad. qui sont très tongrs el très étroits. Les piq. brach. sont an nombre de 1:2 environ et leur long. diminue depuis le premier ventr., qui est beaucoup plus grand que l’art., jusquanu dernier dors. qui égale l'art.; les piq. ventr. sont a pen près cylindr., mais les autressont aplatis et élargis en forme de spalule; tous ces piq. sont très serrés el dressés perpendicul. au bras. Lécaille lenlac. int. esl très allongée, lancéolée et pointue; l'ext. est petile, étroite, arec la pointe émoussée.

La conleur à l'état vivant est d’un brun foncé ou marron; le purtour des boucliers rad. est plus clair : la face durs. du disque est souvent tachetée de petits rercles hlancs ou tres clairs entomrant une partie centr. phus foncée: la face dors. des bras est brunc avee des ammulations plus claires; la face ventr. est blanche ou d'un blane jaunatre. Ces colorations sont conserves dans l'alcool.

L'O. annulosa a surtout été signalée en Méditerranie, à Naples, vers 80 à 100 11. de prof, ; elle a également été rencontrée sur la cóte oceidentale dlirlande,

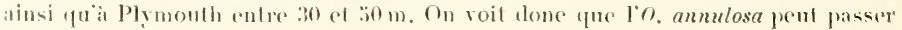

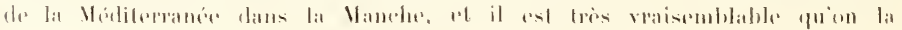


trouvera un jour sur nos côtes, soit en Méditerranée, soit dans l'Atlantique.

L'O. annulosa se distingue très facilement de l'o, aranea par sa taille plus grande, par sa structure beaucoup plus rohusle, et par le nombre de piq. brach.

\section{G. ÉCHINIDES}

\section{OURSINS}

Le test des Échinides est plus ou moins globuleux, parfois aplati, mais à contour esscutiellement arrondi ; il est couvert de piq. entremélés de pédic., et parmi lesquels on peut recommaitre les tubes ambul. disposés en 5 rangées méridiennes doubles. Si lon envisage le corps d'un Échinide tel que le Paracentrotus lividus, loursin comestible de nos coles, qui appartient aux Échinides les plus simples ou Régchiers, on remarquera que ces tubes s'étendent depuis le péristome jusquiau voisinage du périprocte. Le péristome est recouvert d'une membrane molle et assez grande; il offre en son milieu la bouche reconnaissable à ses jo dents proéminentes; lanus est entouré de tress petites plaques courrant unc aire de dimensions restreintes. le périprocte. J animal marche sur sa face orale ou ventr. Le squel. tig. 3 et 4 est constilué par de nombreuses pl. soudés qui comprennent dabord, an pole oppose a la bouche, 2 cercles de chacun 5 pl. cl entourant le péripr. ap ; j de ces pl., plus grandes, sont interrad. b et chacune porte un orif, génil., ce sont les pl.génitales g. 0 ; les 5 autres, sont plus petites et dites ocellaires (r), elles offrent un oril'. plus petil. Ces pl. entourant le péripr. qui s'est substitué à la pl. c.-dors, représentent le squel. prim. de l'Échinide : elles forment ensemble l'appareil apical. Chaque pl. prim. est le point de départ de 2 rangees de pl. lig. 4, ou mieux d'une ringee double disposee suivant un des méridiens du corps el se contimunt jusquau perist. : les j rangée doubles qui font -uite aux pl. ocellinires sont dites radiales a ou ambulacraires et les j autres interradiales ou interambulacraires i : le tout forme la couronne, dont la partie la plus élargie est l'ambitus.

Les pl. ambul. sont percées de pores disposés par paires $\mathbf{d}$, par lesquels passent les canalicules aquifères sourrant dans les Lubes aubul. Chez les jeunes Oursins, chaque pl. ambul. porte une seule paire de pores el par suite un seul tube ambul. Chez les Cidaridie, cette structure simple est conservée, 
mais chez les autres Échinides, les pl. simples se réunissent en pl. plus grandes dites composies, dont chacune oflre plusieurs paires de po res. Les 2 pores dechaque paire se trouvent dans une petite dépression appelée peripode et partoris ils sont réunis par un sillon, un dit alors qu'ils sont renjugues. Parfois les pores sont simples. Les pl. interambul. sont plus grandes el plus larges que les ambul., et elles ne sont jamais composées; en principe, elles ne portent pas de pores. Toutes les pl. sont munies de tubercules servant a larticulation des piq. on des pédic.. et quon appelle, suivant leur taille, primaires, secondaries, on milinires. Les pl. ambul. de la première paire quiltent la ranges a laquelle elles apparticument et passent sur la membrane buec. formant s paires de pl. bucrales.

Les pl. interambul. qui limitent le perint. prénentent, sulf chez les Códaridir, une encoche, l'entuille peristonienne, par wi passe une branchie externe, petil diverticulum ramifié de la cavité générale. Jes pl. ambul. et interambul., au nombre de 20 qui limitent le pourtour du périst. se retroussent en dedans du test pour former une bordure saillante, la ceincure pérignathique fig. 65 ; de plus. 10 de cespl.. tantrit les ambul., tantót les interambul. se soulevent chacune en une apophrse saillante, l'unricule: lorsquelles proviennent des pl. ambul., les 2 auricules de ehaque paire comvergent et se soudent pour former une sorte diarche fermée: lorsquelles viennent des pl. interambul. Cidaridae, elles restent simplement arcolées liune a l'aulre.

La ceinture pérignathique el les auricules domment inserlion aux muscles d'un appareil masticateur très compliqué appelé lanterne fig. 65 et 66 et qui comprend de nombreuses pièces. Les plus importanter on michoires fig. 66 ,

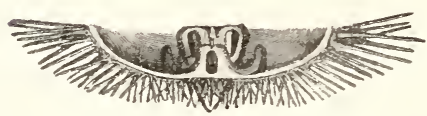

Fin. 65. - Parlie inferiesure du lest diun Oursin aree l'appareil masticaleur (l'apres R. Pенів:

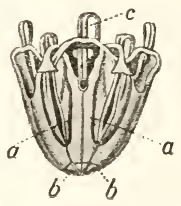

Fig. 66. - Ippareil masticateur isolé (d ajrés R. Perн1єн) ; a. machoire ; b, dents; c, plume.

a , ont la forme d'une prramide triangulaire, dont le sommet lourné vers le bas porte une dent pointue $\mathbf{b}$ qui est visible de l'extérieur fig. 65 et 66 ) el qui ofre, sur sa base, me piece molle et recourbée, la plume c). Ces mâchoires s'accolent les unes aux autres par 2 de leurs faces, la 3 e, ext., est libre; elles sont creuses el la face ext. offre pres de sa base une grande ouverture, la fenétre externe, qui, chez beaucoup d'Échinides, est fermée en laut par 2 épiphyses soudées l'une à l'autre: mais il peut arriver que les ejpiphree-ne se soudent pas et la tenetre ext, reste des lors ouverte en haut. 
La lanterne comprend encore dintres picces et clle constitue un appareil complexe qui fournit des caractéres tres importants, surtout pour l'élablissement des familles: elle nexiste d'atleurs par ches lous les lichinides, et ceus qui en sont pourvus sout dits Ginalhostomes.

Les piq. sarticulent a l'aide de muscles sur un tubere. du test dont les dimensions dépendent de celles des piy. lespuelles sont très variables. Les tuberc. sont lisses ou crenelés, perfores ounon. et le-plus gros sont souvent esutonrés d'une dépression arrondie, le cercle scrobiculare. Tee piq. prim. des Cidaridae, appeles radioles, sont tres grands: dans le g. Dorocidaris, ce sont des baguettes exlindriques atteignant 10 cm. de long. sur 3,5 à f mm. de larg.; dans le g. C'entrostephunus, ils sont aussi très longs, mais extrem. minces el creux; ailleurs ces piq. sont cylindriques avec l'extrém. plus ou moins ubtuse, on en forme d'un cone très allongré et très pointu, ou encore ils se montrent aplatis en spatule, etc.

Les pédic oflrent une structure très varieje; saul de très rares exceptions, ils possedent 3 valves : celles-ci oflrent une partie basilaire, ordin. courte et élargie ligr. 67 b), terminée infér. par des saillies servant à l'articulation aree les autres valves (1), et un limbe général. allongé et élargi en forme de cuilleron bl. On en distingue 5 sortes: les pédic. tridactyles ont les valves très allongées et le limbe plus ou moins élargi pouvant mesurer 2 ou 3 mm. de longueur 1 ; les ophicephales 2 , beaucoup plus petils el qui se montrent spécialement sur la membrane bucc., ont les valves courtes et fortes et munies à leur base d'un arc calcaire articulaire très développé ; les trifoliés (3) sont très petits et constitués par des valves aplaties et minces; les rostrés, spéciaux aux Irréguliers, ont des valves étroites, légèrennent recourbées et ne se touchant quà leur extrém. Enfin les pédic. globifëres 4 sont, au point de vue de la classification, les plus importants : ils sont constitués habit. par des valves minces et étroites portant sur leur face ext. une grosse glande qui s'ouvre au dehors à l'extrém. de la valve (fig. 14 ; chez les Cidaridie, au contraire, la glande est renfermée dans la valve calcaire. Le liquide secrété par les glandes a une action venimeuse sur les petits animaux.

Je signalerai encore les sphéridies, organes vraisemblablenent sensoriels, constitués par une petite tète ellipsoïdale articulée sur un petit tuberc. et formée par un tissu calcaire vitreux, recouvert d'un épithélium cilié. Les sphéridies sont essentiellement situées sur les pl. ambul, au voisinage du périst. Elles manquent aux Cidaridæ; certains $g$. n'en ont que 5 en tout, une par rad.; d'autres en présentent plusieurs.

La cavité générale des Echinides est vaste et le liquide qu'elle renferme est très abondant. Les organes int. les plus apparents sont : le tube digestif et les glandes genitales. I.e premier forme un long tube rattaché a la face int. du test : il ślève rertical. de la bouche en passunt i l’intérieur de la lanterne, puis il décrit, avec des inllexions deux cercles complets, liun ventr., latutre dors., qui se font suite en changeant de sens fig. 7 . Les glandes génit., an nombre de j chez les Réguliers, forment des masses 
importantes qui oceupent les interrad. entre les sinuosités du tube digestif. Les sexes sont séparés; lee glandes miles ont une couleur orangée, les femelles sont plus pales; chacune d'elles suure an dehors par le pore que possède chaque pl. génit. Ce sont ces glandes qui forment la partie comeslible des Oursins, du moins chez le Paracentrotus lividus de nos coles. la seule espèce utilisée dans l'alimentation.

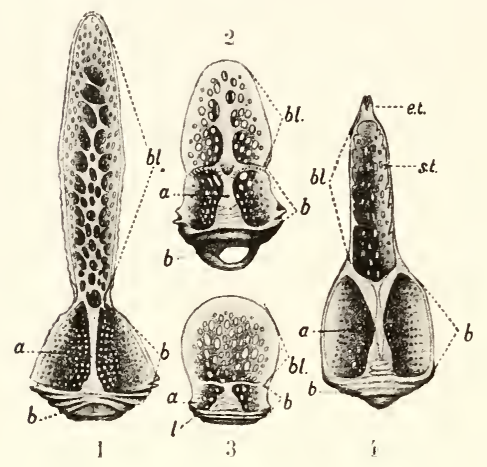

Firi. 67. - Diverses formes de valves de pédicellaires: 1 , tridactyle; 2 , oplicéphale: 3 , trifolié ; 4, globifère; bl, limbe; $\mathbf{b}$. partie basilaire séparéc en ? moitiés par l'apophyse a: st, bords denticulés du limbe du pélic. globifère; ec. orifice terminal: 1, surlaces artieulaires (d'après Montexnas).

les Ghinides rampent sur leur face ventr. plus ou moins aplatie qui sapplique sur le fond sous-mariu. lantot sur le sable ou le gravier, tantiot sur les rochers: d'autres fois ils sont enfouis complètement dans le sable ou dans la vase. Les uns se meuvent a l'aide de leurs tubes ambul. extensibles et contractiles, terminés par une vent. el sur lesquels ils se hàlent en quelque sorte; d'autres progressent grâce à leurs piq. : beaucoup d'Oursins Irréguliers marchent vaiment sur les pointes de leurs piq. ventr., et les C'idaridie s'accochent aux corps élrangers à l’aide de leurs longs radioles.

Les Oursins pourus de michoires se nomrissent d'Algues ou d'autres vétrélau marins; cenx qui en ont dépourvus avalent simplement le sable un la vase dan-laquelle ils vireut et se nourrissent des particules alimentaires que celle vase renferme.

Les Echinides se divisent en deux groupes naturels:

Les Échinides Réguliers chez lesquels le test offre un contour circulaire et dont la forme globuleuse se rapproche de celle d'une spliere plus ou moins aplatie sur la face orale: la bouche el l'aums sont diamétralement opposés; c'est à eux que sappliquent surtout les caractères généraux que je viens de résumer.

Les Échinides Irréguliers chez lesquels le test est aplati et dont le contour n’est plus circulaire, mais devient ovalaire; l'anus n’est plus opposé à la

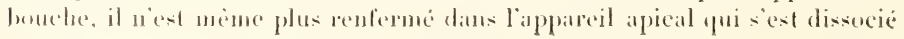


pour lui livrer passage: quittant le pole apical, il a subi une migration le long de l'interrad. post. 5 et se trouve dès lors placé sur le bord post. du corps ou même sur la face ventr. Cette migration de l'anus le longr d'un interrad, a fait disparaître la glande génit. qui s'y trouvait, ef ces gl. ne sont plus quau nombre de 1 ainsi que les orif. génit. qui leur correspondent. En outre, les ambulacres de la face dors. subissent me modification lres particulière : les zones porilères partant du prole apical s'écartent les unes des antres sur une certaine partie de lem trajet, puis se mppochent de nouveau en englobant entre elles une portion médiane en forme d'ellipse allongée. On a compréé la tigr. ainsi formée sur la face dors. du test à une fleur avec ses pétales, et on a domné le nom de pétales aux rég. ambul. ainsi entourées par les 2 séries de pores, d'abord divergentes et ensuite convergentes.

Les Échinides Irréguliers actuellement vivants se répartissent en 2 ordres :

Les CLIPÉASSTRIDÉs fig. 87 chez lesquels la bouche occupe le centre de la face ventr., et qui possèdent un appareil masticateur comparable ì la lanterne des Réguliers, mais plus simple.

Et les SPATAVGIDÉs fig. 88 à 97 chez lesquels la bouche noccupe plus le centre de la face 'ventr., mais se tronve reportéc en avant ef qui manquent complètement d'appareil masticatoire.

I.es Clypéastridés ne sont représentés sur nos cotes que par une seule espèce de très petite taille. Les Spatangidés renferment plusieurs espèces et offrent différents caractères particuliers qu’il est nécessaire de résumer. Le contour de leur test est ovalaire: le corps est plus ou moins aplati et la face ventr. est plane; la bouche se trouve à peu près à égale distance entre le centre de cette face ef le bord ant. du test; l'anus est situé sur le bord post. Le périst. est ovalaire transvers. et garni de petites plaquettes : son bord post., saillant, appelé la lèrre inf., constitue une sorte de petite pelle pouvant s'enfoncer dans le sable ou la vase où vit l'animal; lorsque celuí-ci marche, la lèvre s’enfonce dans le sable qui pénètre ainsi peu à peu et automatiquement dans le tube dig.

Au milieu de la face dors. se trouve l'appareil apical comprenant 1 pl. génit. et 5 pl. ocellaires: la pl. madrép. a, comme d'habitude. envahi la pl. génit. 2, mais le plus souvent elle s'allonge vers l'arrière el atteint l'interrad. 5 . De l'appareil apical partent les 5 ambul., mais il n'existe plus ici que 4 pétales, carle radius ant. III n'a pas les zones porifères écartées et il est général. creusé en gouttière ; les pétales forment donc une étoile ì 1 branches. A la face ventr., l'interrad. post. 3 a subi également des modifications profondes; la plaque qui forme la lèrre inf. est impaire et s'appelle le labre; les pl. suivantes, paires, courrent un espace allongé appelé le plastron sternal. Les zones ambul. ventr. II et IV quilimitent ce plastron sternal sont général. assez étroites; ce sont les avenues ambul. ventr. Il est très important dans la classification de noter la long. du labre par rapport aux pl. ambul. voisines. 
Le test est garni de tuberc, général. plus petils sur la face dors. que sur la face ventr. L'ne disposition spéciale aux Spatangidés consiste dans l'existence de bandes très étroites qui s’étendent sur certaines rég. du test et qui sont couvertes de très petit- piq. vibratiles ou clasules; ces bandes s'appellent fascioles : il en existe plusicurs sortes el leur importance est très grande pour la clasification et la détermination des g. On distingue suivant leur trajet lig. 68 : Le fusc. sous-anal a qui limite en dessous du péripr. une aire ovalaire transr. appelée le plustron sous-anal; le fasc.

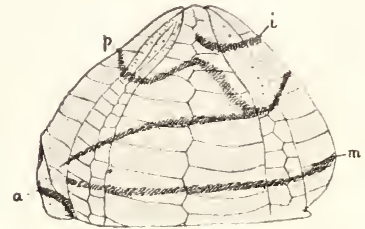

Fig. 68. - Vue latérale scluématrque d'un Échinide In'ténlier pour montrer les principales formes de fascioles : $i$, fasc. interne; p, lase. pripiptate; m, fasc. marginal; a, fasc. sousanal d'aprés Dfrariz: et HéHOIARD? péripétale p qui contourne extér. les pétales et fournit souvent une branche lat. second. qui se dirige en arrière vers le péripr; le fusc. interne (i) qui entoure à une certaine distance l'appareil apical et (oupe les pétales dont il fait disparaître phus ou moins la pointe proxim. Le fasc. le plus fréquent est le fasc. sous-anal. Lorm a donué le nom de Prymodrsmuxs anx formes qui le possèdent et il appelle Prymadètrs ceux qui possèdent un on plusieurs des autres fasc.; les g. dépourvus de fasc. sont dits Adètes.

J'ajouterai que les tubes ambul. subissent aussi des modifications profondes chez les Spatangidés suivant les rég. du corps que l'on considère. An voisinage de la bouche, les tubes donnent naissance à de nombreuses branches scond. et prennent une forme en pinceau, ils ne possèdent qu'un pore minique entouré d'un large péripode; au pourtour de l'anus. ces lubes ont égal. une forme en pinceau, mais ils possèdent une paire de pores. Les lubes du rad. impair ant. ont une forme en rosette. Enfin les tubes des pétales sont très élargis et forment des sortes de branchies munies de 2 pores chacun, tandis que sur le reste du corps les tubes restent extrêm. pelits, coniques et ne possèdent qu'un seul pore. Tous ces caractères sont utilisés dans la classification.

Détermination des Échinides. Ia détermination des Échinides Irréguliers ne présente aucune dilliculté. La forme ditlère beaucoup d'une espèce à l'antre el les fase. smut bien ifparents sur l'animal encore muni de ses piq. Le spatungus se distingur, par sa couleur violet pourpre, des autres espèces de nos mers qui sonl grises : seul parmi nosesperes françases, le Sahizaster canaliferus n'a pas de fascinle sum-anal) enfin l'Fithinocyamus pusillus se

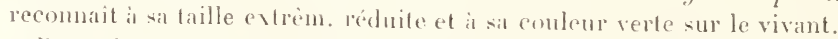

Parmi les Réguliers, la dislinclion entre les ('illaridir 2 espèces françaises et les autres formes se fait il premiere vue. Ia détermination de ces dernières est plus délicate el comporte ¿z examens dilférenté : celui de l'Échinide muni 
de ses piq., et celui du test démudé. L'élude du test intact comporte non seulement eelle des piq., mais anssi celle des pédice : il lant d'abord édudier ces derniers entiers en les montant dans le bamme on dans la wlyérine, ponis examiner les valves isolées ef lissociées: je conseille pour ce ternier examen de faire boutlir sur la lame alle-mème on dans une ciapsule les pédice aver

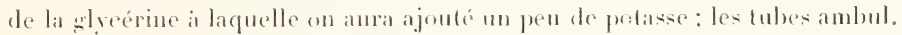
ef la membrane buec. semnt étudies de la mème maniere pour reconnatre les spic on les pl. quïils penvent renfermes. le lest serit déponillé en tout on en partie de ses piq. i l'aide de la potasse bonillante : on examinera surtout les zones ambul. el om comptera les paires de pores que porte chaque pl. et qui sont disposés en ares: on pourra ainsi séparer de suite les g. Echinus, Psammechinus, Spharrechimus et Paracentrolus. On examinera très altenlivement les petits Échindes méditeranéens qui pomratient être des Geno-

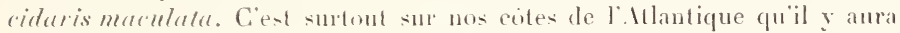
lieu de compter allentivenent le nombre des paires de pores afin de distin. guer le Paracentrotus lividns du Psammechinus miliaris.

\section{TABLEAL DES ESPËCES}

1. Le test est arrondi et globuleux; la bouche se trouve au milieu de la lace ventr. el l'anus lui est diamétralement opposé; le péripr. est entouré par les 11 pl. prin. S.Cl.ÉCHINIDES REGULIERS

- Le test est oval. et plus on moins aplati; la bouche et l'anus ne sont pas diamétralement opposés, et le péripr. n'est pas entouré par les pl. prim. S. Cl. ÉCHINIDES IRRÉGULIERS]. . . 1?

2. Lespiq.prim. rarlioles sont peu nombreux et très grands : ils égalent ou dépassent le diam. du test et ils s'articulent sur des tubere. très gros. Les autres piq., très petits, sont aplatis et ils forment une couronne autour de la base des piq. prim. qui manquent sur les zones ambul. Les pl. ambul. sont simples et ne portent ehacune qu'une seule paire de pores. Les pl, du test se continuent jusquà la houche sur la membrane buccale

Les piq. prim., nombreux, forment au test un revetement uniforme et serré, aussi bien dans les rég. aub. que dans les rég. interanbul.; les pl. ambul. sont "composées " el portent chacune plusieurs paires de pores. Les pl. du test ne se continuent pas sur la membrane buce. qui ne renferme que de petites pl. isolées.

3. Les piq. prim. sont plus lougs que le diam. du test et ils peruent atteindre et mème dépasser le double de ce diam.; ils sont finement striés longilud. Les valves des grands pédic. globif. sont terminées par une forte dent conique el pointue, en dessous de 
laquelle se trouve l'orifice glandul. Couleur grise assez terne.

Dorocidaris papillata p. 108)

- Les piq. prim. égalent au plus le diam. du test et sont munis de stries longit. assez grossières: les valves des gros pédic. globif. sont dépourvues de dents à leur extrém. et l'orif. glandul. est terminal. La couleur est très vive : elle est due en grande partie à la teinte rouge ou rougreâtre des piq. seeond. : les piq. prim. sont grris ou rosés.

Stylocidaris affinis (p. 110

4. Les piq. prim. sunt extrèm. longs et très tins, très fragiles, creux et ils dépassent liurgement le diam. du test; ils offrent des annulations alternativement violettes et blanc-jaunâtre, et sont munis ¿ leur surf. d’aspérités visibles a lieil nu, formant des verticilles: le test est al'une couleur violette très louncée.

Centrostephanus longispinus p. 112

- Les piq. prim. n'alteignent pas le diam. du test et ils sont ordin. beancoup plus courts; ils sont épais, solides, pleins, lisses ou striés, mais dépourvus d’aspérités ou de spinules verticillées .

5. Le péripr. est oceupé par une très grande pl. unique qui en courre presque toute lasurf. les valves des pédic. globif. portent chacune $2 \mathrm{gl}$. et sont munies, en dessous de la dent termin, d'une seule dent impaire lat. Espèce très petite * Genocidaris maculata (p. 115

- Le péripr. est couvert seulement par 4 pl. égales et triangulaires; les piq., très forts, atteignent la moitié du diam. du test; pas de pédic. globif. Couleur très foncée, noire.

Arbacia aquituberculata p. 113

- Le peripr. est formé chez l'adulte par de nombreuses petites pl. inégales; il existe toujours des pédic. whohif. dont chaque valve porte une seule gl. volumineuse et impaire. .

6. Chaque pl. ambul. est formée par la réunion de 3 pl. prim. et les arcs renferment 3 paires de pores chacum.

- Chaque pl. ambul. est formée par 1 ou 5 pl. et les ares renferment 4 ou 5 paires de pores au moins

7. Espèce de grande taille pouvant alteindre ou dépasser $12 \mathrm{~cm}$. de diam. Les valves des pédic. globif. offrent seulement 1 ou 2 dents de chaque coté ; membrane bucc. renfermant de petites pl. fenétrées 「g. Echinusi.

- Espèce de taille moyemne on réduite, le diam. du test ne dépassant pas 6 em. Nombreuse-dents de claque cotédes valves des pédic. globif.: membrane buce. courerte de pl. asséz épaisses et saillantes g. Psammechinus.

๖. Les piq. sont très serrés, nombreux mais assez eomts; chaque pl. interambul. porte plusieurs tuberc. prim. irrégul. disposés; les tuberc. ambul. prim. sont de la même taille que les tuberc. interambul. . 
- Les piq. prim. sout peu serrés et assez longs : il n'en existe qu'un seul sur chaque pl. interambul, et les tuberc, ambul. prim, sont plus petits que les interambul.

9. Le test est plus ou moins conique: il existe général. un tuberc. prim, au milieu de chaque pl. interambul. Les piq. sont colorés en rose lavé de blane et parfois verdìtres ì la base; le teat est d'un rouge tantit uniforme. tantit interrompun par des bandes vertic. blanches. . . . , . . Echimus aculns p.11ti

- Le test est ghobulenx, général. les pl. interambul. noulfunt de tubes prim. que de 2 en 2 pl. Les priq. prim, sont d'un beall vert; le test est foncé, brum on brunâtre.

Echinus melo p. 11s

10. Espèce de petite taille ne dépassant guère 3 a 3.5 cm. de diam.; le test est vert: les piq. sout verts avec l'extrém. rougeâtre : ils sont fins, courts et très serrés. Les pl. interambnl, sont hautes; elles portent chacune un gros tubere. prim. et plusieurs tubere. second. notablement plus petits et asiez espacís; lestubere. ambul. prim. sont plus petits que les tubere, interamb. prim. La membrane buce. est converte, en dehors des 5 paires de pl. buce. prim., de grosses pl. vertes on rerdatres épaisses et imbriquées. formées par un tissu cale, compact.

Psammechinus microtuberculatus p. 12.2

- Espèce de dim. moyeunes nunie de piq. forts, épais et asiez courts, gris-verdâtre on brun-verdàtre, ou encore vert: avec l'extrém. violacée. Les pl, interambul, sont contes et leurs tubere, prim. sont tres rappr.; lestubere. second., nombreux, ne sont pas beatcoup plus petits que les tubere prim. Les pl. de la membrane buce. sont rougeàtres el constiluéer pall un résean calcaire.

Psammechinus miliaris 1. 121

11. Test asiez devé, ghbulenx, Chaque pl, interambul, porte phoieurs

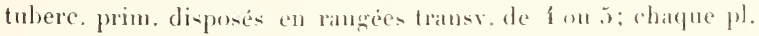
ambul. porte 2 lubere, prim. ì peu prés éganx anx précents. Les pores sont le plus ordin. disposés en ares de 1 paires. les

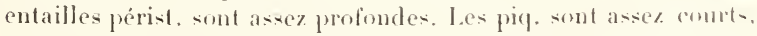
subegans, serrés, ef forment an test un revetement mifurme. le test est violet, les piq. sont violets aree l'extrem. blanche, par-

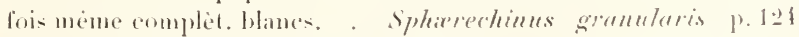

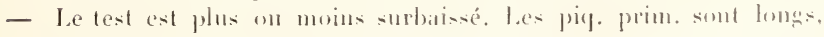
promtus et beancoup plus arands que les piq. serond. Les pores sont disposés en ares de sp parires et parfois meme de ti. Le teat est verdatre; les piq. sont brun-verditre, vert-olivitre, ou d'm vert plus ou moins foncé.

Paracentrolus lividus p. 123

12. Test ovalitre alleignant a peine 1 em. de Jong. mifomément

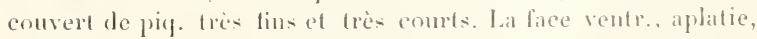
ollre en son centre la bouche, et. en arrière d'elle, l'anus qui e-t 
situé entre le centre el le bord post. du test. Il existe encore un appareil masticatem: les fasc. font complèt. défaut. Comleur générale verte (). Curbenstrumí. Échinoryamms pusillns p. 127

- Espèces de taille plutol grande, i contour ovalaire aree la face ventr. aplatie. Labouche est reportée en avant du centre de cette face, entre celui-ci el le bord ant. du test, tandis que l'anns se

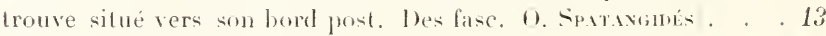

13. En fase. sous-anal: í orif. gén. . . . . . . . . . . 14

- Pas de fasc. soms-anal, un fase. péripétale durquel se détache un fasc. lat. L'appareil apical est reporté très en arrière. Vú de profil, le test, qui est très élevé dans la rég. post., va en s'amincissant rapidement et devient tres bas vers son bord ant. L'ambulacre ant. imple est transformé en mu sillon très prof. avec bords vertic.: 2 orif. génit. . Schisaster canaliferus (p. 1:8

14. In fasc. sous-anal seulement. Le test est corliforme; la face dors. offre, dans les interrad. lat., quelques grands piq. allongés, dirigés en arrière el recourbés, portés par de gros tuberc.; les autres piq. sont beancoup plus courts. Conleur violette.

Spatangus purpureus 13. 129

- Enplus du fase. sons-anal, il eviste m fase. lat. on m fase. int. Couleur grise. . . . . . . . . . . . . . . 15

15. Un fasc péripélale en plus du lase. sons-anal. . . . ; . . 16

- Un fase. int. en plus du fase. sons-anal. . . . . . . . . . 17

1r. Fasc. péripétale en forme de lyre: les pétales post. plus courts que les ant. Liappareil apical se tronve vers le le milien du test.

Brissopsis lyrifera p. 132

- Le fasc péripélale a des contours sinueux aver des angles rentrants et saillants, et il présente surtout un angle rentrint très marqué dans les interrad. lat. de la face dors.; lapp. apical est reporté en avant. les pétales post. sont un pen plus courts que les ant.

Brissus unicolor p. 133

17. L'ambul. ant. est plus ou moins fortement déprimé et forme un sillon arrondi qui sétend, tantôt de l'app. apical., tanteit seulement du fasc. int. jusquan bord ant. du test.

- Imbul. ant. mon déprimé el restant ì fleur du test

18. Le sillon ant., tres large, commence à lapp. apical, el il se troure traverse, dans sa parlic ant., par le fasce int.: les faces ant, et post. du test sont arrondies et it pen près de mène haul. ; le test

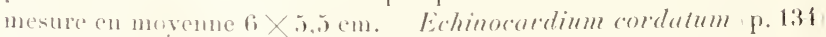

- Ia pirrlic de lombul. ant. enmprise en dedans du fase int sur la

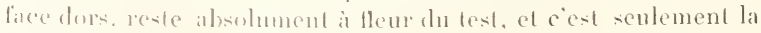

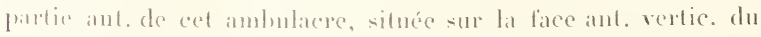

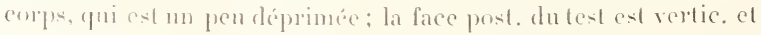
un pou plus baute que la face ant. Le corps est à peu près anssi 
Inng que large et ne dépasse général. pas $1 \mathrm{~cm}$.

Echinocardium mediterraneum p. 135

19. La face dors. offre un certain nombre de gros tubere, portant des piq. plus longs que les autres, surtont dans les "2 aires interrad. ant. et de chaque coté de l’ambul. ant., et qui se montrent égal.. mais moins dévetoppés. dans les interrad. pust. Le finse. int. est petit. Esp. de pelite taille ne dépassant gnère 3 cm. de long.: test très fragile. . . . Echinocaidium flasescens p. 136

- Il n'existe pas de gros tuberc. surles interrad. ant. dor's., on, s'il en existe, ils sont peu nombreux et localisés vers le bord ant. du

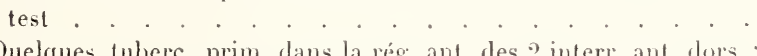

20. Quelques tuberc prim. dans la rég. ant. des 2 interr, ant. ders.; le labre est assez long quoique plus large que long et il atteint le niveau du hord ant. de la 20 pl. ambul. vois. Lespece est plus grande que l'E. flusescens et peut arriver it 5 et mème $5,5 \mathrm{~cm}$. de long. Le fasc. int. n’est pas très développé.

Echinocardium mortenseni p. 137

- Il n’y a jamais de gros luhere. dans les interrad. ant. dors. et le revetement de piq. reste tout is fait uniforme dans les 5 interrad. Le fare. int. est allongé et il s'étend en arant plus loin que che\% l'E. morlenseni. Le labre est extrèm. court, très élargi et il ne dépasse pas le milien de la première pl, ambul. voisine. Le test. assez grand, peut arriver jusqü ì 8 cm. de long.

Echinocardium pemuatifidum p. 138

\title{
S. CI. EGHIVIDES REGLLIERS
}

\author{
F. GIDARIDÆ Agilseiz ET Desor.
}

Le lest est globuleux; las pl. ambul. of interambul. \&c prolongent sur lat

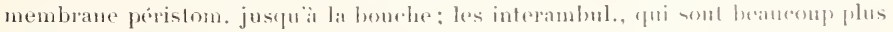

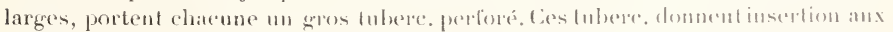

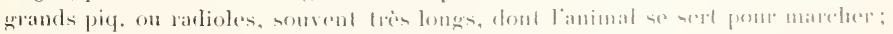

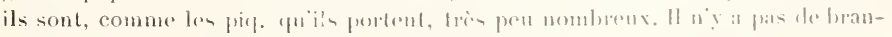

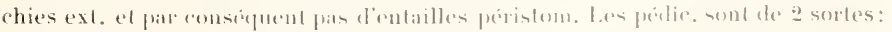

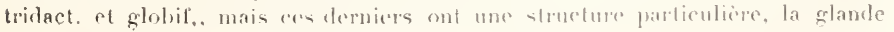


étant enfermée dans la valve calcaire au lieu d'être portée par elle; la tète du jédic. sarticule directement sur la tige qui est composée de 2 parties : une sup. plus courte, la tigelle, et une inf. plus longue, la hampe ; souvent à l'union de ces 2 parties se trouve une collerette de bagueltes pointues. Les sphéridies font défaut. La lanterne ollue, à sa partie sup., des organes particuliers, les organes de Stewart, sortes de diverticules de la memlmane entomant la lanterne el qui se

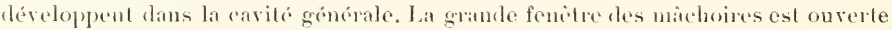
en hant, les cpiphyses restant tres courtes: les dents oflrent un sillon sur leur face int. La cemture perignathique est forme d apoplayses fommies par les pièces interamlut., ot les amricules onvertes ne se rejoignent pas au dessus des canaux ral. Colte disposition est an relation avee l'atal simple des pl. ambul, qui noffrent jamais quine seule paire de pores chacune ef ne se sondent pas en pl. comprosées. la test démuli présente un aspect très caractéristique arec ses gros lubere, interrad, entourés chacun d'un large cercle scrobiculaire.

\section{G. DOROGIDARIS A. AGISSIZ,}

Les piq. sont trés longs el leur long. peut égaler 2 fois le diam. du test; ils sont quelquefois lisses, mais le plus somrent munis de stries longit. Les grands

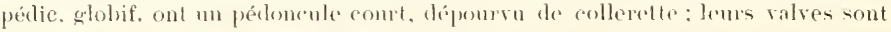

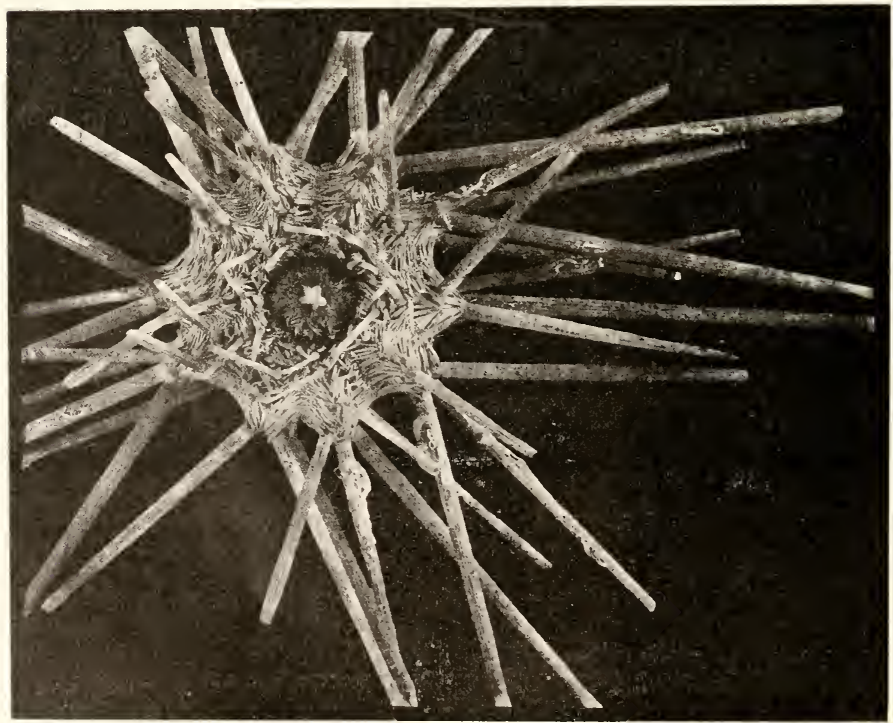

Fir, 69. Dorocidaris papillata arec ses piquants, face rentrale, $X 23$. 
terminées par une forte dent recourbée et l'orif. de la glande n'est pas foul it fait termin. Les pelits pédic. globif. sont égal. munis diune dent lomin. : les pédie. tridact, ont une strueture simple: les spienles des lubes ambul. sont en forme de $\mathrm{C}$.

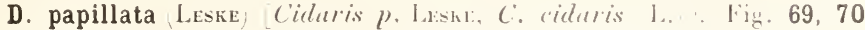

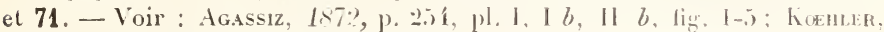
1883, p. 113 ; Mortexsex, 1903, p. 31.

La taille est assez grande; le cliam. du lest, dépouillé des piq., peul atteindre $45 \mathrm{~mm}$. et les plus grands rachioles ont 85 a $90 \mathrm{~mm}$, (le long. D)ans: l'exempl. que je représente ici fig. 48 , le diam. du tesl. est de fi3 $11 m$. el lit hauteur de $23 \mathrm{~mm}$; le diam, de lappareil apical est de 21 mm. el celui du périst. de $15 \mathrm{~mm}$. Lesstries des radioles sont constituées non pas par des saillies continues, mais par une série de tubere, allongés, lrés petils el très rapprochés. L’appareil apical est assez grand, les pl, génil. soml pentagronales. Le péripr. a un contour pentagonal avec les cotés légèr. excavés. Le périst. a les bords onduleux ; il est couvert de pl. imbriquées, beancoup plus larges que longues el épaisses. Les grauds pédic. whbil. ont un pédonrule tres cont, leur tete ext trè renllie et lesvalves penvent atteindre 1.5 $\mathrm{mm}$. de longueur: les bords de l'oril. glandulaire sonl garnis de dents (71, a : ces pédic. se montrent strtout sur le péripr. Les petils pédic, globif. onl la meme struclure générale b , mais la dent terminale est plus petite, leurs valves

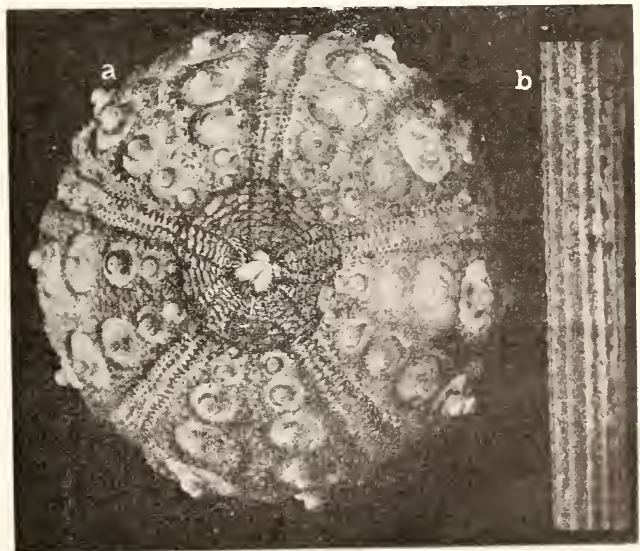

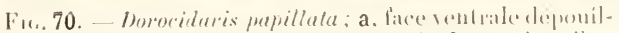
lie des piquants, légirenent gromi: b. portion d'un piquant, $\times: 3$.

\section{sonl plus étroites}

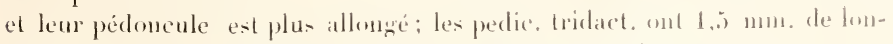
gueur, avec un pédoncule asiez longr el des valves élroiles.

La couleur a l'état vivant esl d'un blane grisilre lave sourent de jaune paille, mais jamais brillante et plutot assez terne.

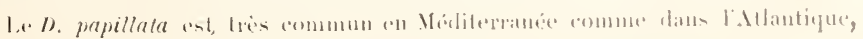


mais il rit toujours a une certaine prof. et ne se rencontre guère au dessus de $50 \mathrm{~m}$. Diatre part il ne dépasse général. pas 3 à $409 \mathrm{~m}$. On le troure principal. dans les fonds vaseux et les pêcheurs le capturent assez fréquemment. It remonte jusquanx coles de Norvege ef descend jusquaux Canaries ; on le retrouve égal. sur les cotes des litats-lnis el anx Intilles.

\section{G. STYLOCIDARIS NoHTENex.}

Le g. Shylocilaris differe du g. Lorociduris par les radioles plus courts et ne dépassant pas le dian. du test et par les gros pédic. globif. dépourvus de dent terminale, tandis que lem pédoncule est muni d'une collerelte. Les petits globif. ont une dent terminale.

St. affuis Pmump cidaris a. P'm.. Fig. 72 el 73. - Voir : Montexsex, 1!)(13, p. 35, pl. 1, figr. 1. et 1!)13, p. 11.

Laspert extérieur du S\% affinis est bien différent de celui du D. papillata: les radioles sont trés court e et lem long. ne dejasse pas le diam. du test qui varie entre 3 et ím. La striation de ces radioles est plus marquée que chez le D. pupillata : elle est constiluée par des granules grosiers disposés en rangées longit. Lexlrém. des valves des gros pédic. globif. n'est pas armée d'une dent el forifice, armondi. est toul a latit term. 72, a ; lorif. des petits globif. at plus petit et plus etroil que chez le $D$. papillata b. Les pédic. tridact. paraissent faire délaut dans les indiv, méditerranéens, tandis qüils existent dan ceux des Etats-Lnis ou les gros ghobil. sont en revanche tres rares.

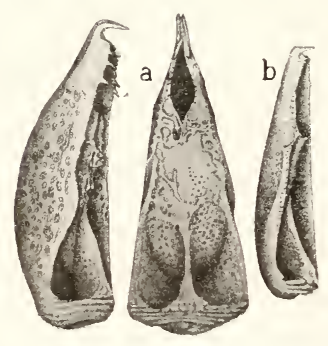

Fic. 71 . Dororidaris papillate: a vilves d'un gros predice. globifere; b. d'un pelit globifiere, $X 30$ dingès NontexsLy),

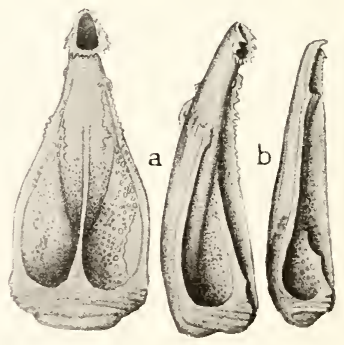

Fit. 72. - Stylocilaris affinis; a, gros glolofíre: b, petil globifère, $\times 30$ (d'aprés Montessex.

La couleur est trés brillante, d'un rouge vif: les radioles sont brunatres avec des bandes plus claires el plus foncées ; ces colorations sont conservés dans l'alcool. Cofte livrée élégante permet de distinguer facilement le 


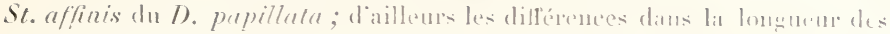

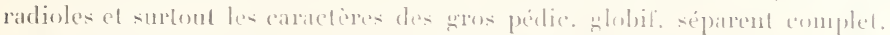

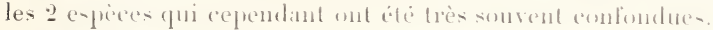

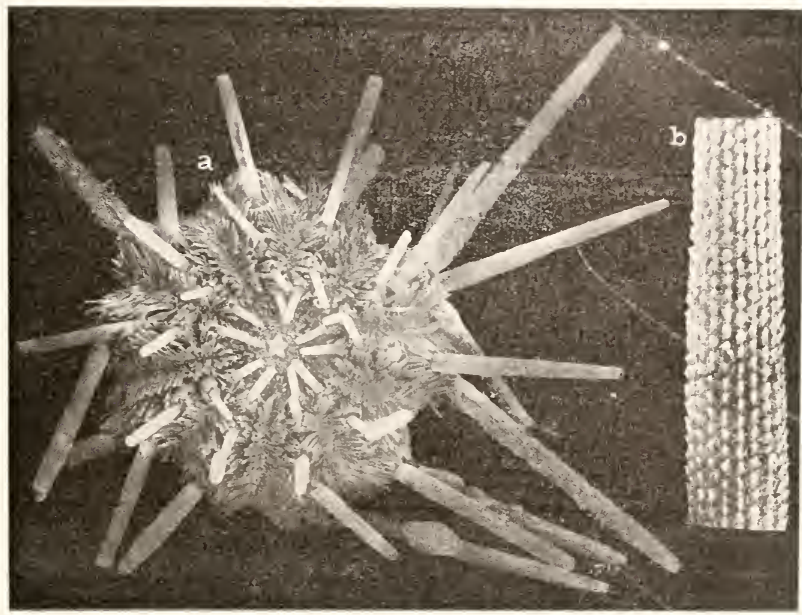

Frg. 73. - Stylociduris uffinis; a, fice venlade, légèrement réduil; b, piquant, $X \dot{t}$.

Le St, affinis se troure sur nos cotes de la Mediterranes it une prof. de $30 \mathrm{~m}$. principal. dans les fonds coralligines et il preut deseendre juspuia $1: 50 \mathrm{~m}$, : il a élé rencontré a Villefranche el it Yaples. Dans l'Vtlintique, il nest connu que sur les coles d'Afrique el aux iles du Cap leet et il ne parail par remonter plus Jaut que le détroit de Gibraltar; on te retroure aux Antilles.

\section{F. GENTRECHINID屟 JACKsoN.}

Le test est le plus somrent aplati sur la faer ventr. : les ple ambul, sont composées ", el portent plusieurs paires de pores. Les pl. ambul. el interambul. s'arrètent au périst. qui est courert par une membrane nofrant que "j paires de pl. principales isolées el général. pen déveloplus. Il "xiste des branchies ext. dont la présence determine 10 entailles péristom. Le's pl. ambul. el interambul. portent des lubere. prin.. ot lem piqf. qui restent toujours assez étroits, sont nom- 
breux et forment an lest un revetement serré el compated qui a fait comparer les Oursins à des Iférissons ou it des Chataignes. Les peidic. appartiennent aux types ophicéphale el trifolié et il sy ajoute le jus sous. des pérlie. tridaet. et

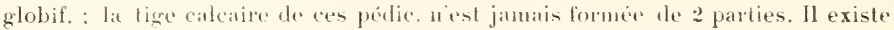
des spluéridien. Les auricules, formées par les pl. aubul., se réunissent en une arche au dessus thes cananx rad.

\section{G. GENTROSTEPHANUS PETEHS.}

Le test est solide et aplati. Les pl. ambul. et interambul. portent chacune un gros tuberc. prim. perforé, crénelé et entouré d'un assez large cercle scrobiculaire. Les zones porifères sont étroites et les pores sont disposés en ares de trois paires L'appareil apical et le péripr. sont relativ. grands et les pl. ocellaires ne touchent pas le péripr. Les piq. sont tries longs, très fins et lragiles, verticillés, ereux, et dans l'espèce françaire, leur long. dépasse beaucoup le diam. du test. Les pl. interambul, voisines de lappareil apical portent de petits piq. claviformes qui, d̀ l'état vivant, se font remartpuer par me couleur rouge rif et par leur monrement rotatoire continuel ; il existe des pédic. tridact., trif. et ophic., plus des globif. d'un type particulier. Les spicules des fubes ambul. ne sont pas en C, main de forme irreg.g. en II, en V, en V ou en T. Les dentsoffrent un sillon sur leur face int.

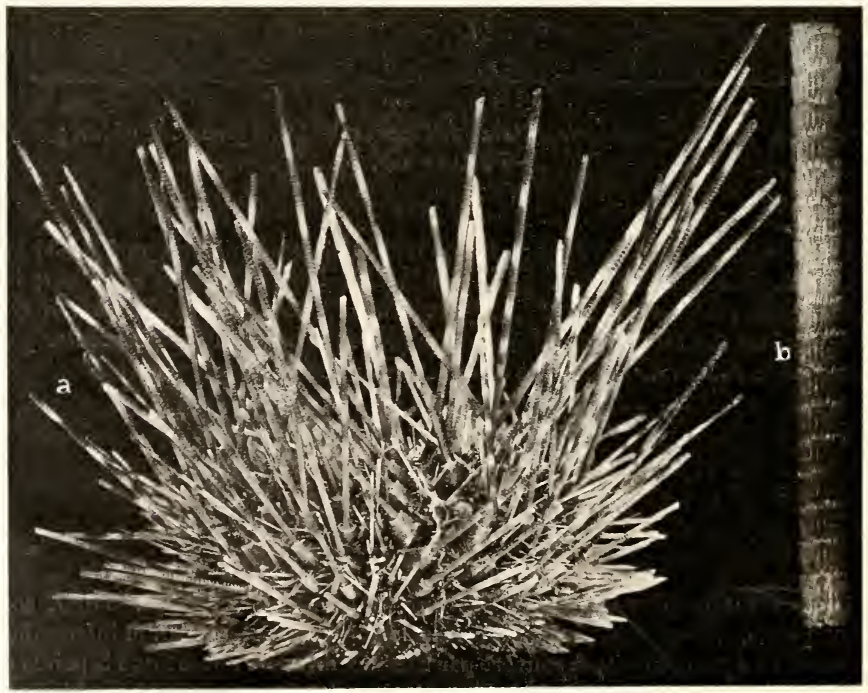

Fi6, 74. - Centrostehanus plongispinus; a, animal cntier vu de cóté, grandeur naturelle; b. piquant, $\times: "$. 


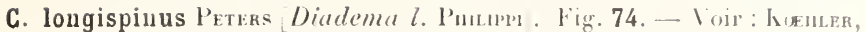
1895, p. 25, pl. IX. fig. 4 ; et 1!m!), p. 220, pl. XXXI, fig. 20.

Je renvoie pour la deseription detaillée du test is mon travilil de 1895 . Les piq. prim, dans un exempl. dont le diam. est do 38 mm. comme celui que je représente ici, ont $60 \mathrm{~mm}$. de long. Ils alleignent leur plus grande long. à l'ambitus et décroissent rapidement à mesure quion s'approche du périst. ; à l'ambitus et au dessus, ils sont dressés vertic. Ces piq. présentent de larges annulations alternat. blanches ou jaunatres et violelles; ils sont presque incolores sur la face ventr. On distingue très facilement à la loupe, à la surf. de ces piq., les verticilles caractéristiques forniés par des spinules serrées avec la pointe libre, et qui déterminent une striation longit. Les piq. second. sont très fins. Les pédic. tridact. ont la tête très allongée et les valves, étroiles, mesurent 3 mm. de long.; les trif. ont les valves 2 fois plus longues que larges. Les globif. sont très particuliers: leurs valves sout atrophiées et ils portent, sur leur tige très courte, 3 glandes qui en occupent à peu près toute la long.

Le tég. qui recouvre le test est d'un brun violacé assez foncé, le milieu des aires ambul. et interambul. est marqué par une ligne clatire qui s'arrète vers l'appareil apical.

Le $C$. longispinus est assez rare et il reste localisé dans quelques points ; en Méditerranée il a été surtout trouvé à Naples, sur des fonds coralligènes, vers $50 \mathrm{~m}$. Il a été capturé sur nos côtes à des prof. même moindres, à Nice à Toulon et à Carry, mais assez rarement. On l'a signalé aux Açores, aux Canaries et sur les côtes du Maroc.

\section{G. ARBACIA Grav.}

Les pl. ambul. sont composées et les zones porifères sont étroites sur la farc dors., mais elles s'élargissent sur la face ventr. à mesure qu'on se rapproche du périst. : les tubere. prim. sont imperforés et lisses. Le péripr. ovalaire est courert par † grandes pl. triangulaires. Les piq. prim. sont très forts, épais et assez lougs. Il existe des pédic. ophic., mais très rarement des pédie. trifol. et tridact. Les pl. sont recouvertes d'un système de granules, ponctuations, etc., anquel on a donné le nom depistroma et qui est plus ou moins developpé. Les épiphyses des mâchoires sont courtes et ne se soudent pas au dressus de la grande fencitre; les auricules restent ouvertes.

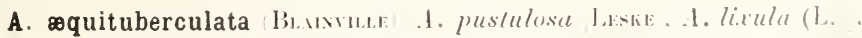

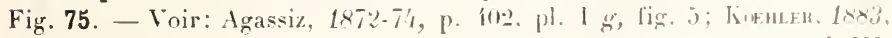
p. 117 , et 1911, p. 234, pl. XIII, fig. 1-6, Lowr ; 1857, p. 1115 et 110, pl. 111 .

Le test reste de dimensions moyemues, il est parfois un per surbaissé, plus souvent un peu conique; la lace ventr. est aplatie. Liexempl. que je reprisente ici a $45 \mathrm{~mm}$. de diam. et $25 \mathrm{~mm}$. de haut. Les zones ambul, sont étroites, mais en dessous de l'ambitus elles augmentent rapidement de largeur et elles prennent une apparence pétalö̈le en raison de l’élargissement de-paires de 
pores qui sont an, nombre de 3 par pl. Chaque pl. ambul. porte un gros tuberc. prim. Les zones interambul., tres larges, portent à l'ambitus 4 à 5 gros tuberc. prim., très rapprochés el méne contigus, mais ils se séparent à mesure quion siapproche du péripr. el lépistroma se développe davantage dans leur's intervalles. Les tuberc. seenul. nexistent pour ansi dire pas et

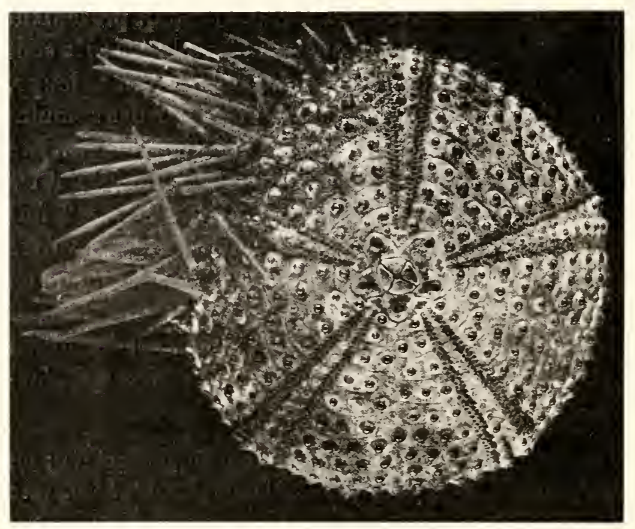

F16. 75. - Arbacia inquiluberculata; face dorsale. légèrement grossie.

les tuberc. miliaires sont pen nombreus. Le périst. est tres grand avec un eontom anduleux, sans entailles. Les piq. primi., grands et forts, sont allongés, assez épais a la hase, pointus a l'extrém.; leur long. peut atteindre la moilié du diam. du lest, mais elle est général. un peu plus petite. Les pédic. ophic. sont de tailles diflérentes et il existe de pe-

lits tridact. Jes spicules des tubes ambul. sont peu abondants. mais de lorme caracte-ristique : ce sont des batomnets élargis en leur milieu ou se tronvent quelquer perforations. Il existe 5 sphéridies en tout, une dans chaque rad.. logée dans une petite niche près du périst.

Les piq. sont tout à fait noirs et les tég. sont remplis de granulations très forcées qui domment à l'animal une coloration d'un noir absolu.

Cette espèce est essentiellement littorale, et rare sur nos còtes méditerranéennes; elle a été rencontrée parfois à Narseille, à Carry, à Niolon, associée au Paracentrotus lividus; elle est plus fréquente du eòté de Nice. Elle devient très abondante sur nos cotes d'Algérie et se retrouve dans plusieurs localités de la Miditerranée. Elle deseend sur les cotes occidentales d'Afrique et a été signalée aux Açores, à Iladère el aux iles du Cap Vert. C'est une forme de mers plutôl chaudes.

\section{G. 'GENOCIDARIS AGAssiz。}

Le péripr. est rècouvert par une très grosse pl. arrondie, arec 3 ou \& petites pl, insignifiantes sur l'un de ses bords. Iar membrane péristomienne noffre 
aucune pl. en dehors des 5 paires de pl. luce. prim. Les zones ambul, sont larges et chaque pl. porte 3 paires de pores. Les pl. du test présentent des fossettes et des impressions diverses qui leur dounent une apparence spéciale. Les valves des pédic. globif., au lien de porter une glande unique comme c'est la règle, en portent 2 chacune et leur limbe, an lien de se recourber en une gouttière ouverte, forme un tuhe ne laissant libre quine serie de petits orif. Les indiv. sont toujours très petits.

* G. maculata Agassiz [Temnechinus m. Ag.), Arbacina pallaryi Gac-

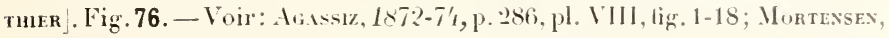
1913, p. 12, pl. 1, fig. 11 el 12; Dönerless, I9lki, p. 198, pl. XXV, fig. 2 el 13; KoeHler, 190\%, p. 226, pl. XXXl, fig. 3.

Le diam. du test varie ordin. entre 6 et $8 \mathrm{~mm}$., les exempl. ayant $10 \mathrm{~mm}$. sont très rares. La face dors, est trés bombée et la face ventr. est aplatie; la haut. est plus grande que la moitié du diam. Chaque zone interambul. renferme $12 \mathrm{pl}$. portant chacune un tuberc prim. vitreux et brillant; le reste de la pl. est recouvert de tuberc. second. nombreux et serrés. Ces pl. offrent des fossettes disposées en étoile autour des luberc. prim., et. entre ces tuberc. des sillons horiz. Les pl. ambul., au nombre de 11 dans chaque zone, portent un tuberc. prim. accompagné de tuberc. second. serrés; elles offrent égal, des fossettes en étoile et des sillons horiz. L'appareil apical mesure à peine le tiers du diam. du test et la moitié ou le tiers est occupée par le péripr. Celui-ci est recourert par une très grosse pl. bombée, brillante, en dehors de laquelle se montrent 3 ou 1 pl. extrèm. petites, parmi lesquelles śoure l'anus qui est done très excentrique. Les pl. génit. sont plus longues que larges, contiguës, et elles portent quelques tuberc. second. assez serrés. Les pl. ocellaires sont beaucoup plus petites et portent égal. quelques tuberc. second. Le périst. est plus petit que la moitié du diam. du test. Les 5 paires de pl. bucc. se trouvent plus rapprochées du centre que du bord de la membrane bucc.; elles portent de nombreux pédic. ophic. Les piq. sont trè courts et les plus grands n'atteignent à pas la moitié du diam. du test; ils sont lisses avec l'extrém. arrondie; les plus petils s'elargissent vers leur extrém. qui est tronquie et offre une petite pointe centr. entourée de

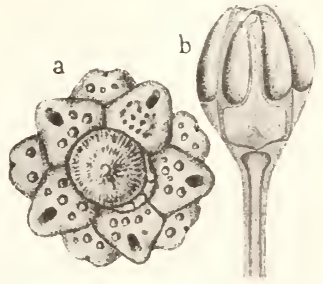

Fiti, 76. -. Tienocidaris maculata; $\mathbf{a}$, appareil apical, $\times$ : (d'aprós \Gassiz); b. pédicellaire globifère, $\times 50$ (d'apres Homrases). dents.

La couleur des indiv. virants est rerte arec des taches claires ou blanches surla face dors.

Le $G$. maculata doit ètre très répandu en Méditerranée, mais il n'it pas été souvent cité parce qu'on l'a confondu arec des jeunes d'autres esp., notamment

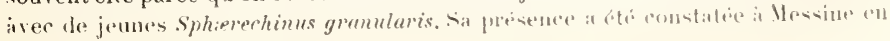


1893, puis il a été trouvé sur les eótes dXlgéric; il est très commun à Naples oì il vit sur les fonds coralligènes a $: 50 \mathrm{~m}$. de prof. En dehors de nos mers, on la reconnu au Congo, a Maulire, aux Agores el sur les cotes de l'Amérique du Tord. Ses limites extrimes en pof. sont 2.2 et $t$ ts m. Les zoologistes qui examineront attentivement les Oursins méditerraneens dont le test natteint pas un cm. de diam. pourront rencontrer le $f$. maculata, quils recomaitront de suite à la gronse p. recouvrant le periper.

\section{G. ECHINUS liONELET.}

Le test est renflé, plus ou moins globuleux: la membrane bucc. renferme de nombreuses pl. fenêtrées en dedans el en dehors des : tubere prim. sont relativ, petits et les pores sont trigéminés. Les valves des pédic. globif,, assez courtes, n'offrent, en dessous de la dent termin., qu'une ou 2 dents de chaque côte; le limbe, court, présente quelques travées transv. qui sélargissent parfois au point de transformer la gouttiere que forme le limbe en un tube offrant seulement queliues trous successifs. Les peid. tridact. sont très grands, arec les valves allongées et étroites, munies de rangées transr. de dents extrêm. fines.

Les 2 g. Echinus et Psammechinus onl, l'un et l'autre, les pores disposés en ares de 3 paires et leur distinction, d'ailleurs très facile, est surtout fondée sur les caractères des pédic. : dans le g. Psammechinus, les valves des pédic. globif sont munies de plusieurs dents lat. el elles sout dépourvues de traves transv. (fig. 81, a), celles des pédic. tridact. sont assez grandes; tandis que dans le g. Echimus, les globif. n'ont qu'une ou 2 dents lat. et leur limbe otlre des travees transvers., les pédic. tridact. ont les valves minces et allongées. An point de vue praticgue, il faut noter que nos 3 especes françaises du g. Echinus sont de très grande taille, leur diam. dépassant $12 \mathrm{~cm}$. a l'́btat adulte, tandis que les 2 espèces tu g. l'sammerhinus sonl, l'une de petite laille et spéciale à la lléditerranée, et l'autre, de taille moyenne, son diam, ne dépassant guère "̈ cm., propre à l'Atlantique. les tubes anbul. renferment, dans les $2 \mathrm{~g}$., des spicules en C.

E. acutus L.мияск. Fin. 77 et 78. - Voir: Agassiz, 1×7:-7', p. 159,

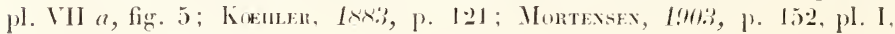
fig. 4, 7 et $8, \mathrm{pl}$. II, ete.

Ie dian., qui est habit. de 8 a $12 \mathrm{~cm}$, arrive facilement a 15 . Le contour est arrondi le lest est parfois un peu globuleux, mais le plus ordin. il est nellement conique vis par en haut, son contour est circulaire. Les pl. ambul. ne portent général. pas toutes un tubere prim., mais ces lubere. se montrent de 20 en 2 pl. senlement et celat avec quelques irrizularites les tuberc. secoud. sont peu nombreux el disposés sans ordre. Itans les zones interambul., chaque pl. porte, en principe, un tubere, prim. asiez gros et il est rare quon trouve une interruption dans la rangée régul. formée par ces tuberc.; les second. sont peu nombreux au dessus de l'ambitus, mais en dessous, ils devienuent plus nombreux el plus gros, el lendent à former des ran-

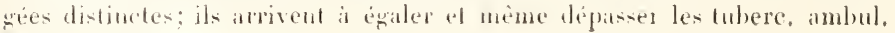




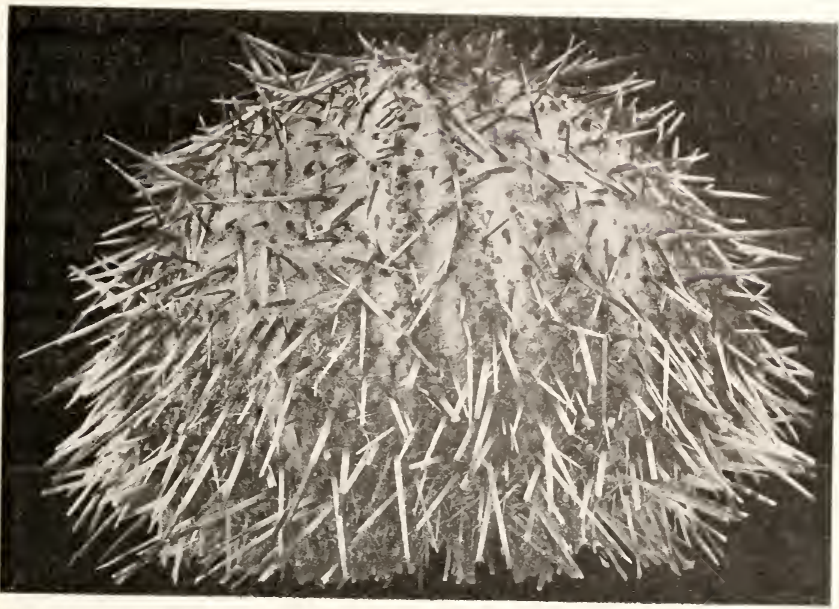

lig. 77. - Echinus aculus; animal entier muni de ses piquants, vu de colé; légèrement réduit.

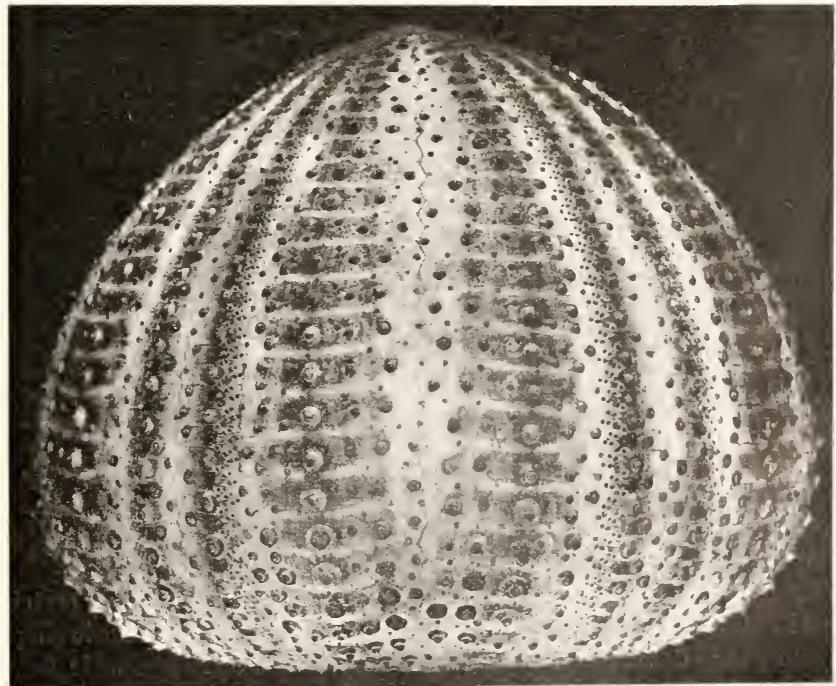

Fı, 78. - Échinus acutus; test dépouillé, vu de còté; légèr. réduit. 
prim, du mème niveau. Les piq. prim, ne sont pas très forts el les plus longs mesurent $25 \mathrm{~mm}$, en moyenne. D'une manière générale, les piq. sont peu serrés et peu nombreux au dessus de l’ambilus et deviennent beaucoup plus nombreux et plus serrés sur la face ventr.

En principe, res piq., sanf cenx de la liace venlr. qui sont presque toujours blanes, sont romger el verts, verts i la base, pmis rouges avee l'extrém, blanche, mais il y a de nombrenses variations. Le lest dénudé a une couleur générale rouge, fautot uniforme, tantot interrompue par des bandes blanches.

L'E. acutus présente une extension géographique très vaste, depuis la mer de Barentz jusquau cap Bojador, et il vit à des prof. trés différentes, depuis 20 jusquà $1280 \mathrm{~m}$. Sur nos côtes, on le rencontre à la fois en Méditerranée et dans l'Atlantique, princip. dans les fonds vaneux où il est très commun à partir de 20 it $2.3 \mathrm{~m}$.

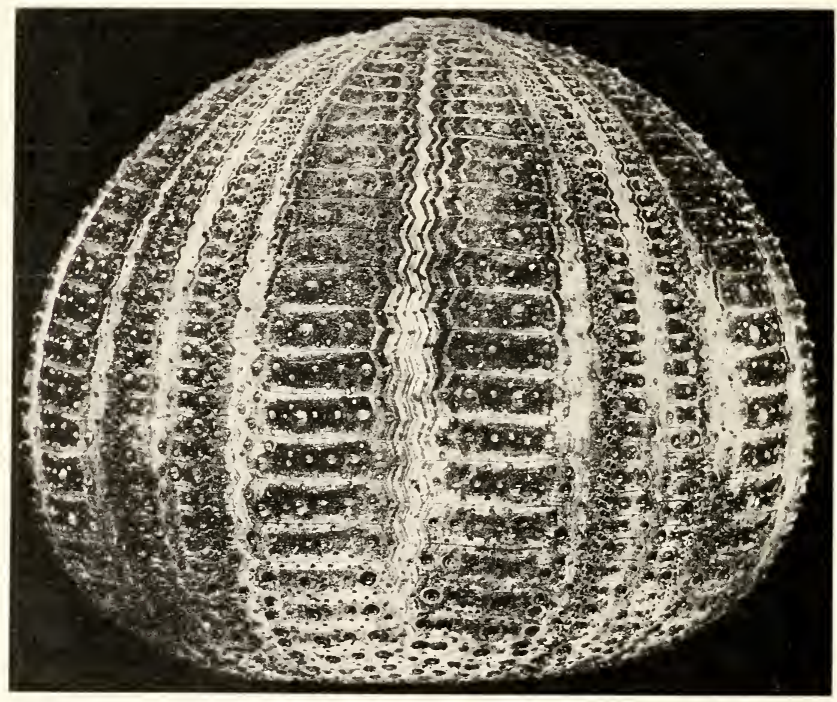

FIG. 79. -- Echinus melo; vu de côté, $\times 23$.

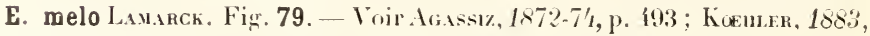
p. 120 el 1895, p. 20, pl. IX, fig. 1 et 2 : Монтахsix, 19113, p. 15 s.

L'E. melo a élé très souvent confondu avee l'E. acutus. D'abord certains auteurs ont donné autrefois le nom d'E. melo à l'E. arutus, sous le simple prétexle que ce dernier álaul lrès gros, il représentail évidemment la forme que bamarck avait voulu comparer à un melon. Il s’est mème lrouvé des 
auteurs plus récents qui ne eroyaient pas a l'existence de l'E. melo paree qu’ils ne l'avaient jamais vu; mais lorsqu'ils ont pu l'étudier, ils ont convenu de la validité de celte espèce. L'aspect est en eflet tout dilférent. L'E. melo a le test globuleux, renflé etventru avec la face ventr. pen déprimée, el il n'est jamais conique. L’exempl. que je représente ici mesure 10,7 cm. de diant. sur $8,5 \mathrm{~cm}$. de hant. Vu par en haut, le test a mentome neltement pentagonal, le milieu des zones interambul, étant un peu aplati a l'ambitus et les zones ambul. un peu proéminentes. Les lubere interambul, prim., au lieu de se succéder régul. sur ehaque pl.. ne se mentrent que de 2 ent 2. Les pl. ambul. sont moins hautes et relativ. plus nombreuses que chez l'E. aculus.

Les piq. prim. Ont une coloration générale vert foncé assez vive; le te-t dénudé est plutôt brunâtre et le milieu des aires ambul. est plus clair; la ligne en zigr-zag qui occupe la suture médiane de chaque zone est bordée de chaque coté d'une série de bandes alternat. blanches et brunes, au nombre d'une demi-douzaine en dessus de l'ambitus el moins nombreuses an dessous. Jes pédic. et les spicules offrent les mèmes caractères que chez l'E. uculus. l.es échant. de petite taille présentent téjà, d'mue manière très nette, les caractères de l'adulte.

L'E. melo est plus rare que l'E. acutus. Sur nos côtes de Provence, il se troure surtout sur les fonds rocheux, vers $30-30 \mathrm{~m}$. de prof., et les pêcheurs le rapportent parfois accroché à leurs entremailles lorsyue ces engins ont balayé le fonds à la limite de la broundo; au large de Varseille, il est assez commun vers la Cassidagne. Du côté de Niee, il devient plus aboulant et parait même plus fréquent que l'E. acutus. Il vit égal. dans l'Atlant. (còtes du Portugal, Açores), mais il ne paraît pas exister au $\mathrm{N}$. le la péninsule ibérique.

E. esculentus Linvé E. sphaera O. F. Mülrer]. Fig 80. - Voir : Agassi,

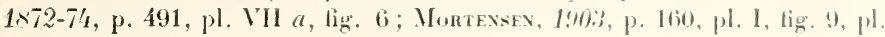
III, fig. 3, etc.

L'E. esculentus est bien différent des z espéces précédentes et il mériterait presque d'en être séparé génériquement; il s'en distingue immédiatement par les nombreux tuberc. prim. qu'il offre dans les zones interambul. et ambul., tandis que le test non dénudé est recouvert de piq. nombreux, serrés, forts et plutot courts, atteignant lous à peu près la même long. et formant au test un revêtement dense et unif.

Le test est globuleux ou parfois un peu conique avec la face ventr. plus ou moins aplatie. Les zones ambul, offrent à l'ambitus ume rangée très régul. de tuberc. prim. immédiatement en dedans de la zone porifère et qui se montrent général. de 2 en 2 pl.; celles qui en sont dépourves offrent un tuberc. un peu plus petit situé vers leur milieu. Les pl, adambul. portent chacune plusieurs tuberc. prim., mais ceux-ci ne lorment général. pas de rangées vertic. ou horiz, rég. ; on remarque cependant vers le milieu de chaque pl. un tuberc. dont la taille est égale ou à peine sup. à celle des voisins 
au dessus de l'ambitus, mais qui, en dessous de l'ambitus, devient un peu plus gros et forme avec ses congénères une rangée longit. distincte qui se continue vers la bouche. Le reste de la pl. interambul. est couvert de tuberc, prim. disposés irrégul., dont te nombre peut atteindre une douzaine, ct an milien desquels se tronvent des tubere. second. et miliaires. Les

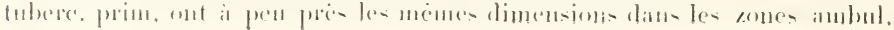
el inlevimbul.

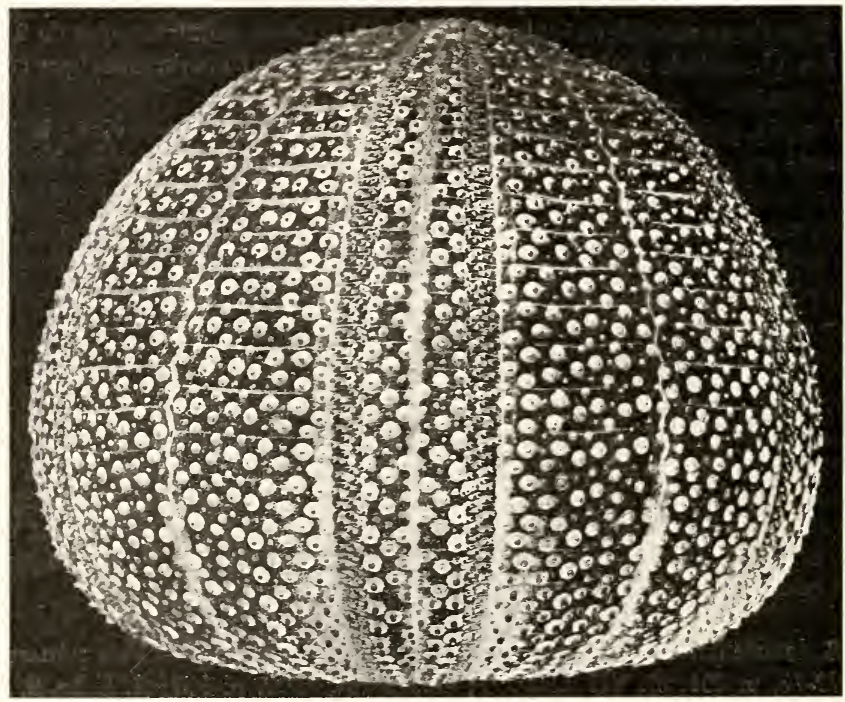

Fśr. 80. - Echinus esculentus; vu de cóté, $\times 13$.

La couleur du test i l'état virant est d'un gris violacé ou rougeâtre, les piq. sont rosés, blanc grisittre ou gris violacé clair.

1.E. esrulentus n'existe pas en Méliterranée, ot cest par erreur que quelques auteurs l'ont signalé dans eette mer : il vit exchusirement dans l'Atlantique où il est d'ailleurs très fréquent, ainsi (jue dans la IIanche, à une faible prof., ( $10 \mathrm{a} 15 \mathrm{~m}$.), et mème à Roscotl on l'a trouvé parfois it la còte lor's de très grandes marées; il peut descendre jusquau deli d'une centaine de m. mais il devient de plusen plus rare. Son extension géographique est vaste: il est en eflet très répaulu sur les

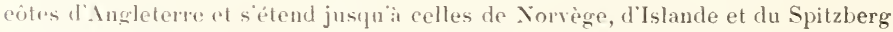
où il peut descendre ì une grande prof. $(126 \mathrm{fm}$. $)$ : d autre part il s'étend jusque *ur les côtes du Porturial.

Malgré son nom, cotle espiece vist mullement comestible. 


\section{G. PSAMMEGHINUS 1.. IgAssiz.}

Les valves des pédic. grobif. prortent de chaque coté plusieurs dents successives; los bords du limbe amincis et non épaissis sont complet. libres en dedans et ils ne sont remuis par aucume travee transs. Les pief. sont nombremx, plutot petits ef

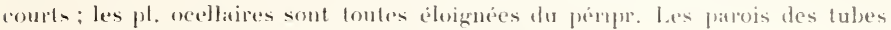
ambul. renferment dis spir. an C: l'oren trighinines.

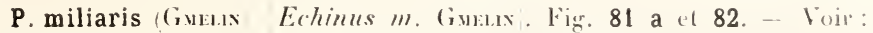

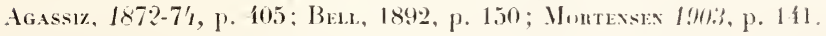

Le test est un peu pentagonal, assez haut, sonvent ligèr. conique, son diam. oscille autour de $50 \mathrm{~mm}$. et $m$ exempl. avant ce dianètre, comme celui que je représente ici lig. 82 , a $28 \mathrm{~mm}$. de haul, ; il est épais et résistant, et remarquable par les nombreux lubere. serrés el subiganx qui le recourrent. En plus du tubere. prim. que porte charne pl. ambul. et interanbul., il existe des lubere, seond. gros el serres, disposes en ranges vertic. et mème horiz. Lappareil apical est assez petit et som diam. mesure 10 mm. ; le périst. est plutit grand et son diam, alteint 18 mun., les entailles péristom. sont peu profondes, larges et arrondies. Lal membrane buce. est couverte, en dehors et en dedans des 5 p. de pl. buce. prim., te pl. allongées, imbriquées, relevees, serrées ef eomstitués par un réseau calcaire grossier. Les piq. sont courts, assez épais, striés longit., aree la pointe émoussée. Ils sont général. d'une couleur verte avec l'extrém. violacée: le test dénudé est gris jaunatre ou brunâtre avec parfois des bandes plus claires verdàtres on blanchâtres.

Le Psammechimus miliaris se distingm très facilement de l'esp. suivante, le P's.microtuberculatus qui nexiste che\% quien Médilerranée et reste beaucoup plus petite, mais conme il a a peu près les mèmes dims que le Paracentrolus lividus, anquel il est parfois associé, il importe d’indiquer leurs diflérences. Deux caractères permottront de séplares de suite les 22 esp. : l" les pores du Ps. miliaris sout toujours disposés en ares de 3 paires, tandis que cluez le Pror. lividus les ares ont au moins 5 paires de pores: 2 les pédic. globif. du Ps. miliaris 81, a offrent

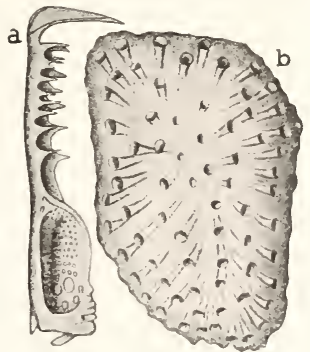

Fit. 81. - Psammerhinus: a. perdiecollare globiliere de P. miliciris. X 130 ; b. plingue lutecale de P. mirmonherenlatus, $X$ in dapres MonTEXSEY? plusieurs dent-le long de lems valves qui smont allongées, tandis que rhez le Par. lividus les valves, tres raccourcies. n'ont qu'une seule dent lat. en dessous de la dent term. 
Le Ps. miliaris est très répandu sur nos còtes de la Manche où il remplace le Par, lividus, tandis que sur nos cótes de l'Atlautique il est souvent associé à ce dernier. C'est une forme essentiellement littorale qu'on trouve à mer basse contre les rocher's, sous les pierres ou même dans le sable. Canluat l'a vu former des exeavations identiques a celles du Par. licidus. On le comnat à Areachon, mais il deseend beanconp plus an S., sur les eótes du Portugal et du Maroc. Il manque en Médilerranée et éest par erreur que quelques auteurs l'y ont signalé. Le Ps. miliaris remonte jusquaux eotes de Inrège et il existe dans toute la mer du Nord a sur les côtes des lless Britanniques: il peut descentre jusquà $60 \mathrm{~m}$. de prof.

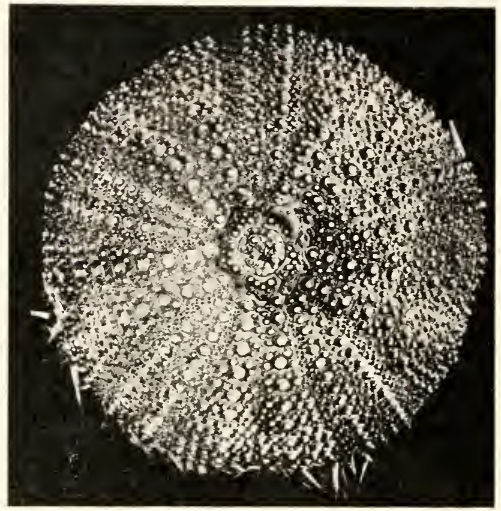

F1G. 82. - Psammechinus miliaris; face dorsale, $X 1$ i.

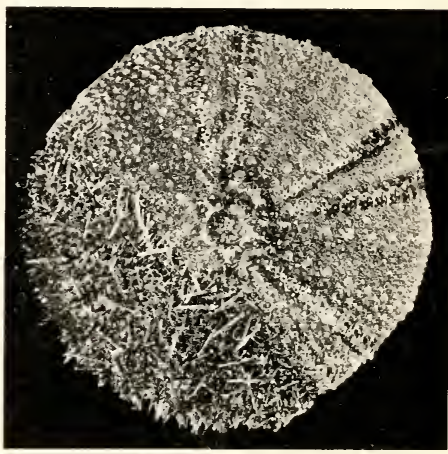

Fig. 83. - Psammechinus microtuberculatus; face dorsale, $X 2$.

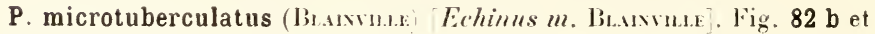
83. - Voir Agassz, 1870-71, p. 191, pl. VI, fig. í: Kumler, 1883, p. 112; Mortinsex, 1903, p. 107.

L'espèce reste toujours de très petite taille et le diam. du test ne dépasse guère $30 \mathrm{~mm}$, il arrive très rarement à $35 \mathrm{~mm}$. Comparé à celui du $P s$. miliaris, ce test se fait remarquer par l'ensemble de ses tubere. plus petits aussi bien les prim. que les seeond. Les tubere. ambul. prim. sont notablement plus petits que les interambul. ; ceux-ci sont assez écartés les uns des autres en raison de la hauteur des pl. ; les tuberc, second. sont beaucoup plus petits. Le Ps. microtuberculatus est essentiellement caractérisé par sa membrane buec. qui, en plus des jo paires de pl. prim., offre un recouvrement assez rég. et très apparent de pl. épaisses, serrées et imbriquées, plus larges que lomgues, assez grandes, surtout en dehors des pl. buec. prim., et plus développées que chez le Ps. miliaris; de plus, au lien d'ètre constituées comme chez ce dernier et chez les autres Échinides, par un tissu réticulé, elles 
sont formées par une masse homogène et transparente, épaisse, traversée par des pores allongés qui forment de véritables tubes, el elles penvent atteindre $1,1 \times 0,7 \mathrm{~mm}$. (fig. $\mathbf{8 1} \mathrm{b}$ Les piq. sont courts, minces, pointus et forment un revètement très serré. Ils ont une couleur verte, vert jaunâtre, ou vert grisàtre; le test dénudé est d'm vert asse\% foncé ou gris verdâtre, avec des bandes plus claires sur les zones porifères.

Espèce très répandue dans toute la Méditerranée, commune sur nos cites an bord des prairies de Zostires et s'y continuant jusqu'it 2:̈ m., pénétrant aussi en "broundo ". Elle a été signalée au Portugal et aux iles du Cap Vert, mais ne parait pas dépasser au $\mathrm{Y}$. la pénininsule ibérique.

\section{(i. PARACENTROTUS YohtFNSE:}

Les pores multigemines sont disposes en ares de :; ou fi paires. Chaque pl. ambul. porte un tubere. prim. landis que les pl. interanbul. en portent quelquesuns qui forment des rangées vertic. très apparentes. La membrane buce. est munie de petites pl. fenetrées en dedans et an dehors des "̈ paires de pl. prim.; les entailles péristom. sont peu profondes. Lne ou 2 pl. ocellaires peuvent atteindre le péripr. mais quéréral. toules en sont exclues. Les pir]. sont assez longrs, forts et serrés. less pédie. globif. n'onl qu une seule paire de lents lat.; leurs valves sont courtes et ranassies et la partie basilaire est plus longne que le limbe : eelui-ci, en forme de gonttière, est dépourvu de traves transw. Les valves des pédie. tridact. sont très longues, la partie lonsilate est très courte, mais le limbe est très allongé et très étroit; il est muni sur toute la long. de ses bords de dents eoniques et pointues.

P. lividus L.ıмırck [S/rongylocenlrotus l. Br.ivit. Fig. 84. - Voir :

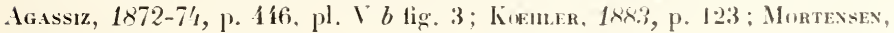
1903 , p. 123.

Les échant. sont de grosseur moyemne et le dian. du test est de 50 mm. en movenne, mais i! pent atteindre une taille mupen plus grande: il est assez déprimé el la face ventr. est aplatie. L'appareil apical est petit mais assez. saillant. Les piq. sont assez longs. forts et pointus. Ienr coloration varie du violet an vert foncé on devient brunâtre ou divatre: le test lui-mème est verdâtre.

Le P. lividus est extrèn. eommun sur nos cotes, prineipal. en Véditerranée oi il est pêché et livré à la consommation par quantités très considérables. C'est surtout à la lisière des prairies de Zostères, à 3 ou $\{\mathrm{m}$. de prof. qu'on le troure en plus grande abondance; il vit aussi tixé sur les rochers, les jetées des ports, ete. Les pêelseurs le eapturent à l'aide d'une longue eanne fendue à l'extrém. en 3 branches. 11 peut descendre jusqu’à $30 \mathrm{~m}$., c. ì d. jusqu’a la limite extrème des prairies de Zostères. Le P. licidus est égal. commun dans l'Atlantique, depuis Biarritz jusquaux eòtes de Bretagne et du Cotentin; il est parfois associé an Ps. miliaris, mais celui-ci domine dans la Manche et finit par exclure le l'. licidus; il est habit. fixé aux rochers à une petite prof., mais at Arcachon on le reneontre égal. dans le sable, assez rarement d'ailleurs. 
On a remarqué depuis fort longlemps, en Méditerranée et surtout sur nos cotes c l'Atlantique, que le $P$. lixidus vivait souvent dans des cavités quil creusait

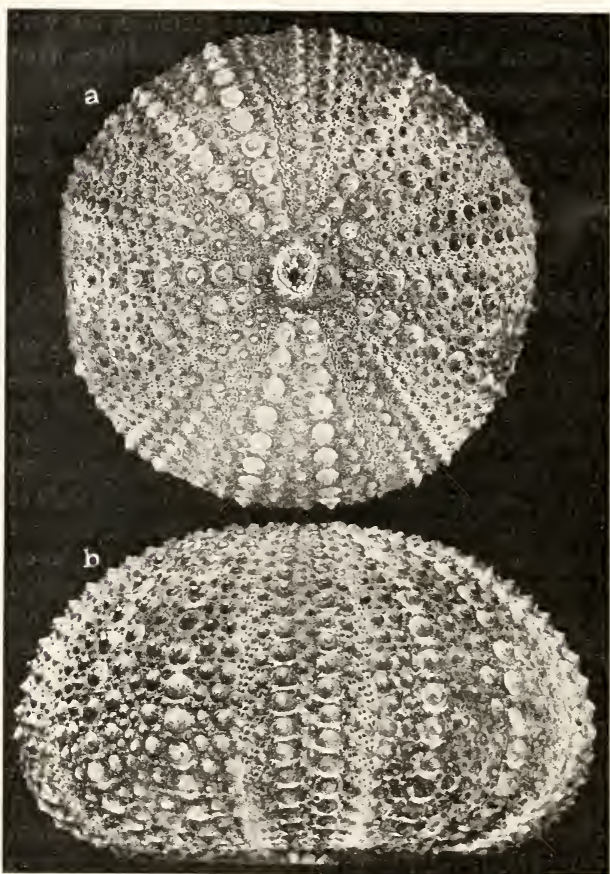

Fic. 84. - Paracentrotus lividus ; a, face dorsale: b, we latérale; légirement grossi. lui-méme dans les rowhers en les usant à laicle de ses piq. grace a des mouvements de rotation. Je renvoin pour artle question an mémoire de C.mlli.al n 'qui a beancoup etudis ces perforatiens. Hans cortaines localiters, les I'. lirilus fixes anx rocher's, ont l'habitude de recourrir lem face dors. à laide dedébris divers, notamment de Kostires, yni les abritent ansi plus ou moins complet. de la lumiere dı jour.

\section{(i. SPHARECHINUS \\ Desor.}

Le test est épais, presque sphérique. muni de tubere. nombreux et imperforés d'égale grosseur dans toutes les zones: le périst., décagonal, est pourvu d'entailles pro fondes. Chaque pl. ambul porte \& p. de pores; la membrane

bucc. mince renferme, an plus de 10 pl. prim, de nombreuses pl. fenétrées. Les piq. sont épais, forls cot courts. Les tètes des pédie. globif. sont très développées et les glandessont volumineuses ; les valves sont terminees par un gros crochet unique el leur limbe est converti, sur toute sa long., an un lube étroit : ces pédic. renferment des spicules recourbés avec l'extrém. un peu elargie, qui se retrouvent égal. dans les lubes ambul.

S. granularis Lamsrci. Fig. 85 el 86. - Voir : Igissiz, 187't, p. 652,

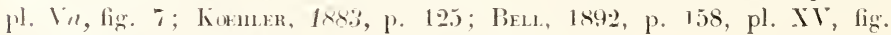
2 el 3 : Мontrisex, 1903 , p. 117 el 1913, p. 11.

Le test alleint une assez grande laille et son diam. est compris entre 60 
et $70 \mathrm{~mm}$, il arrive mème à $80 \mathrm{~mm}$. Il est ghobuleux, avec lit fare ventr. aplatie, tantôt élevé, tantit quelque peu déprimé : l'exempl. que je représente ici fig. 85 . b., mesure $70 \times 5.3 \mathrm{~mm}$. Les fubere. prim, des rones interambul. sont disposés suivant les rangées vertic. et horiz et ils sont it peine plus petits sur les zones ambul. Les piq. forment sur le test un recourrement assez dense ; ils sont serrés et afteignent tou- a pen prè la même long., et ils restent d'ailleurs asse\% courts avee la pointe arrondie, Ces piq. sont ordin. violets avec l'extrémité blanche, et quelquefós ils

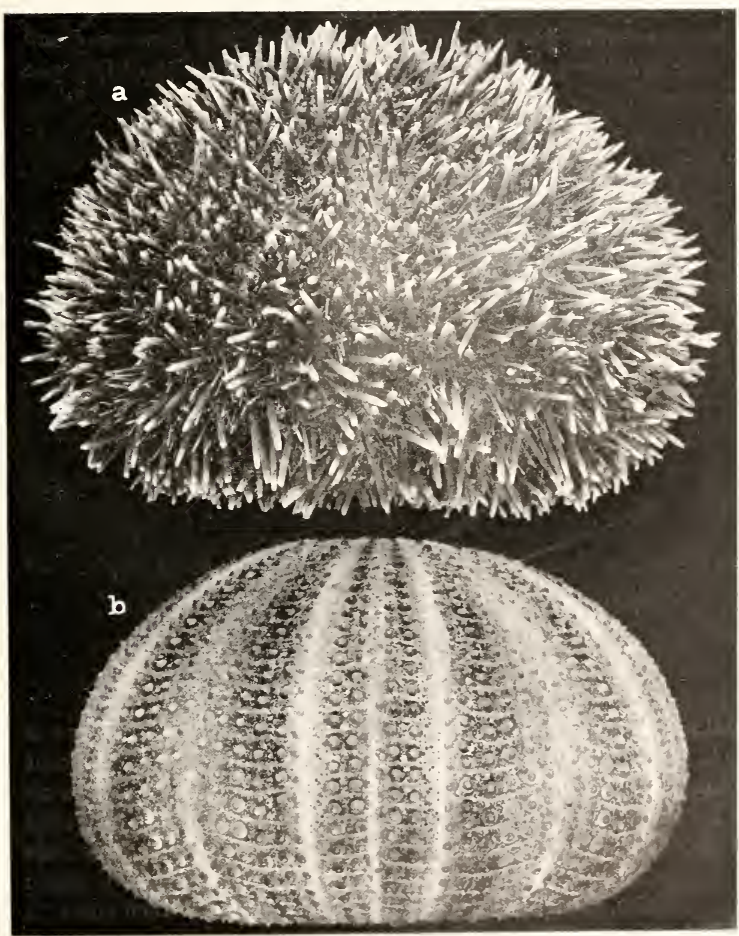

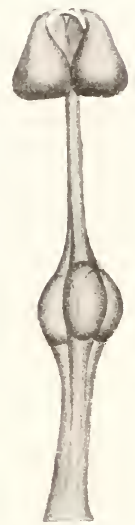

Fini. 86 .

sphierechinus granularis; pédicellatire globifère, $\times$ ti.i.

Fig. 85. - Spharechinus granularis; vues latérales; a, l'animal pourvu de ses piquants ; b, test dénudé; grandeur naturelle.

sont tout à fait blancs; le test est violacé. L.es pédic. globili présentent une structure très caractéristique: le pédoncule porte il quelque distance 
en dessous de la lète, 3 glandes allongées olliant chacune un petit orif. termin (fig. 86. Il arrive assez souvent que ces glandes prennent un développement assez considérable et cela aux dépens de la tète même qui $\therefore$ atrophie el disparait plus ou moins complè.: sur d’autres pédic., cette téte peut se télacher de son pédoncule, continuer it vive el conserver des mouvements pendint un certain temps 1 .

Le $S$. granularis habite surtout dans des sables vaseux à partir de quelques m. de prof. : en Véditerranée, on le trouve sur le pourtour des prairies cle Zostères jusqu sur nos côtes de l'Atlantique dans des stations analogues et à partir de quelques m. de prof.; il peut descendre jusquä $: 30 \mathrm{~m}$. Il a été rencontré dans um grand nombre de local. de la Méditerranée; on le retrouve égal. sur les côtes d'Espagne et sur les cotes oecidentales d'Afrique.

\section{S. CI. ÉCHINIDES IRRÉGULIERS}

\section{CLYPÉASTRIDES}

Ils ne sont représentés dans nos mers que par une seule espèce de fort petite taille, l'Echinocyamus pusillus.

\section{f. echinocyamus Vax Phelsum.}

Le test e'st ovalaire et sa long. atteint tout au plus I cm. ; l'anus est situé sur la face ventr. entre la bouche et le bord post. du corps. Les tubere., petits, sont entourés d'une depression. Il n'existe qu'un seul orif. madrép. à peu près de même taille que les orif. génit. Les zones ambul. sont plus larges que les zones interambul. Les pores de la face dors., disposés par paires, forment des sortes de pétales i bords parallèles. Au roisinage des sutures des pl. ambul., il existe de nombreux pores extrèm. fins qui sont surtont développes sur la face ventr. Il existe en tout "s sphéridies.

(1) Hamano avait, a tort, considéré ces pédoncules munis de leur's 3 glandes et dépourvus de tête, comme des pédic. ophic. modifiés. Une erreur plus grossière a été cosumise par J. Barrols : ayant l'encontré des têtes détachées de ces pédic. globif, il les a prises pour des organismes particuliers qu'il a décrits sous le noun de Trichalina paraloxa; inutile d'insister sur ces erreurs. 
E. pusillus O. F. Müller' E. angulosus Lfske]. Fig. 87. - Voir : Agassi\%, 1872-1874, p. 304, pl. XI e, lig. 3 ; Montexsex, 19197, p. 28, pl. Xll.

Ja taille des exempl. est toujours très petite et les dimensions labituelles sont 8 m. de long., 6 mur. de larg. et $3,5 \mathrm{~mm}$. de hant. On a cité comme long. maxima 1.5 mu, mais ceci est extrem. me. Te corps est ovalaine, plus étroit en avant qu'en arrière: le périst. est grand, un peu allongé, le péripr., plus petit, est situé à égale distance entre le bord pust. du périst. et le bord post. du test. Les pores des pétales dors. sout au uombre de ti il 8 paires. Les pores ocellaires, au nombre de 5 , sont plus petits que les pores génit. qui sont au nombre de í semlement. I.e corps ast revetu miformément de piq. fins, courts et serrés, dont la long. est de 1,5 à $0,8 \mathrm{~mm}$. en moyenne. Jeur coloration est d'un vert somvent très vil qui passe parfois an vert jaunatre. Je renvoie pour les pédic. ophic.. tridact. et tril. au mémoire de Mortexsen.

L'E, pusillus est assez répandu dans la Méditerranée et l'Atlantique. Sur nos cotes de Provence, on lo trouve principal. en dehors des prairies de Zostères, dans les sables

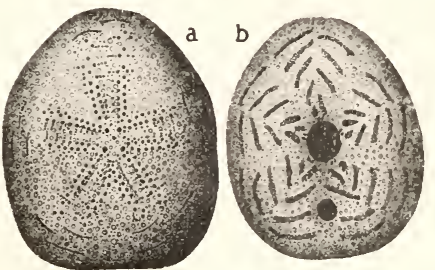

Fur. 87. - Echinocyamus pusillus diaprès llontexsix): a. face dorale; $b$. face rentrale, $X$ i. et les débris coquilliers ou les graviers à Bryozoaires, vers $30 \mathrm{~m}$. de prof.; il peut d'ailleurs descendre plus profondément. Il a été signalé dans un graud nombre de localités de la Méditerranée. Dans l'Allantique, on le rencontre aussi en différents points de nos còtes, généralement dans les sables et les débris coquilliers, dans le "mœrl" de nos côtes de Bretagne, le plus sonvent entre 20 et $40 \mathrm{~m}$. Il remonte dans les mer's du $\mathrm{X}$. jusqu'au cap Bojador. Il peut arriver à une prof. de quelques centaines de 11 . (il a été signalé à des prof. beaucoup plus grandes, mais il s'agissait alors d'une autre espèce'. 


\section{SPATANGIDES}

\section{S. (). PRYUVADETES}

\section{(i. SCHIZASTER Igassiz ot Desor.}

Le corps offre un contour ovalaire; il est déprimé en avant et trés élevé au contraire en arriere; la face post. est tronquéc. Lappareil apical est reporté en arrière. L'ambul. ant. forme un sillon très profoncl, allongé ; les pétales lat. sont itroits, mais profonds : les ant. sont allongés, légèr. infléchis, les post. sont très courts. Le fase. péripétale a un contour assez sinueux; il sélargit en avant et se rétrécit en arrière: vers son milieu, il fournit une branche lat. qui se dirige en arrière, et qui, an niveau du péripr.. s'abaisse rapiel. vers la face ventr., pour se réunir à la hanche oppose en formant un V tres onvert vers le haut. Habit. il n'existe que ? orif. genit.

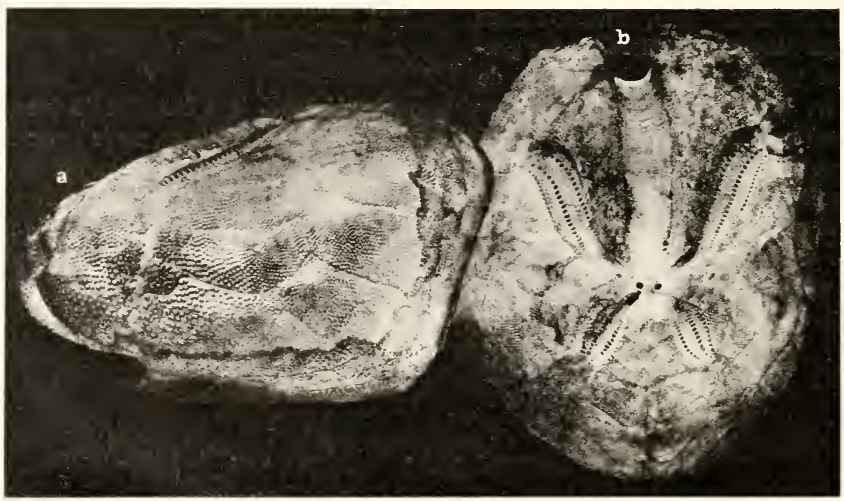

Fín. 88. - Schisaster canaliferus réduit d'un tiers; a, vue latérale; b, lace dorsale.

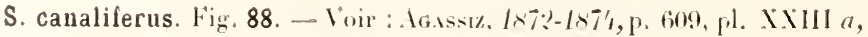

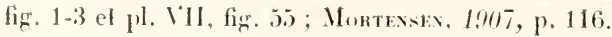

Ja Jotug. pent alleindre $70 \mathrm{~mm}$. ; daus l'indiv. que je représente ici et qui 


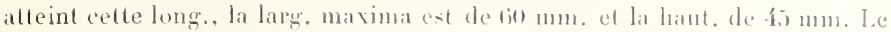
contour du test est ovalaire mais un pen angulenx, avere une forte encuehe en arant qui correspond an silkn ambul. ant., lequel est extrem. probud

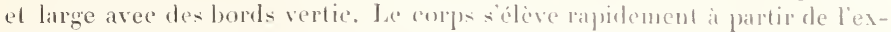
trém. ant. qui est basse, jusqüa l’extrém. pont. qui est tros devée; la lace

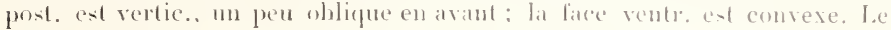
périst. trés roisin du bord ant. du test, ent petit : lat levre post. ent assees próminente el aiguë, el le labre as allongé. Le péripr. est petit, z lois plus haut que large el rapproché du bord sup). du test. leses avenues ambul. ventr. sont plutit atroites tandis que le plastron sternal est large. La lace dors. est uniformément comverte de lubere. fins el rapprochés, qui deviennent plus gros sur la lace veutr., surtout dans la moitié ant. de cette face. Les piq. sont lins, conts el soyeux sur la lace dors. plus lomps sur la face rentr. el ilo ont me lome en spatule sur le plastrom stemal où ils se groupent en 2 toulles divergentes. lesorill. génit. sont au nombre de 2 senlement; toutefois en avant de ceux-ci on trouve la trace de deux autres orif. extrem. rédnits mais il n’existe jamais que 2 glandes génit. en tont.

Les pérlic. appartiement à is sorles : tridact., rostrés, ophic., globile el trif. Je remvie pour leur description à mon mómoire le 188:3, p. 1:37, et a celui de Mortexsex, 1907, p. 117. Je rappelerai senlement, comme je l'ai signalé en 1883, que les pédic. Iridact peuvent posiséder í valves el mériteraient dès lors le nom de tétradaclyles ; on peut mème tromer des pédic. it 5 valves.

Le S. canaliferus est une espeer propre a la Méditerrance. Il est asiez rare sur nos ecotes de Provence et il se trouve dans les mèmes localitis que le spatangus purpureus; il est plus fréquent entre Vice el Jlenton, daus les foncls vatsens de

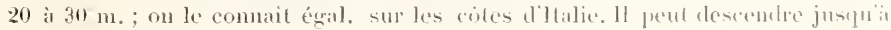
$100 \mathrm{~m}$. de prof.

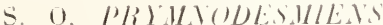

\section{(i. SPATANGUS KLEM.}

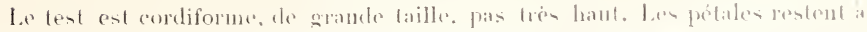

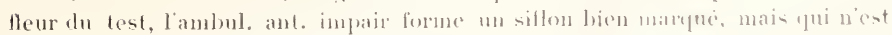

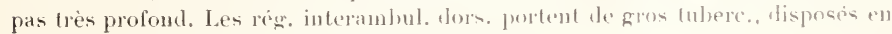
ares successifs. Ln fase, sous-inual serulement.

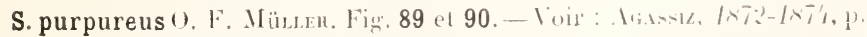

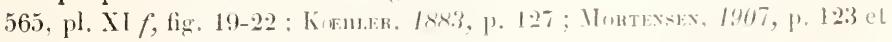
$1913, \mathrm{p}, 2 \cdot 2, \mathrm{pl} .11, \mathrm{fig}, 2-1$. 
Le test atteint facilement 11 i $12 \mathrm{~cm}$. de long. sur une larg. un peu mointre ; il est cordilorme avec un eontour régul.; la lace ventr. est aplatie. L'appareil apical est reporté un peu en avant: le sillon ant. est large. Les pétales sont pointus el movennement élargis, les ant. sont un peu plus longse el forment ensemble un angle plus obtus que les post. qui sont un peu plus étroits et plus rapprochés. l.e péripr. est allongé transvers. el le fase. sousanal, qui est fortement excavé du colé dors., ne renferme que deux tubes ambul. de chaque eoté. La bouche est large et la lère inf. peu proéminente. Les gros tuberc. des aires interrad. dors. sont placés près du bord apical des pl. et forment des séries successives en forme de l tress ouverts qui n'atleignent pas le pourtour du terl. les bords du sillon ant. présentent phusienrs rangées de luberc. un peu phus gros que les voisins; lout le reste de la face dors. est couvert de petits tubere. Ceus de la face rentr. sont plus

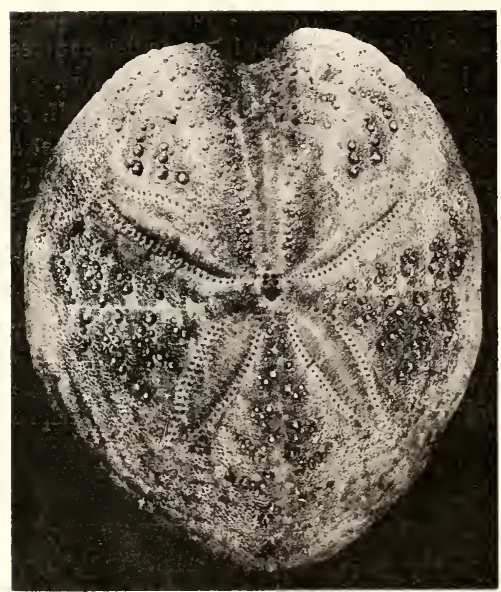

F1g. 89. -. Spatangus purpureus dépouillé des piquauts, $\times 23$; face dorsale d'un échantillon à corps allongé. gros, mais ils se réduisent vers la périph. du test el passent insensiblement aux dors. Les piq. portés par les gros lubere. te la face dors. sout lres longs, pointus, légèrement recourbés; les autres sout plus petits, mais leur dim. augmentent sur la face dors. les pédic. tridact. sont de 2 sortes: chez les uns la tète peut atteindre jusqu’a 2 mm. de long. et les valves sont amincies; les autres ont la tête plus petite. Les pédic. trifoliés sont très pelits et leur limbe est allongé, linement dentic. sur les bords; lesophic. sont rares et n'existent que chez les jeunes, surtout sur la face ventr. Enfin les pédic. globif. sont extrèm. rares et ils ne paraissent exister que dans les petits échint. : le limbe forme

t11 Inbe qui se termine par quelques dents.

Lanimal vivant est t'un violet pourpre assez foncé: la coloration, unilorme, intéresse a la fois les piq. et le test lui-mène: elle se conserve dano l'alcool.

Le $S$. purpureus n'est pas rare dans le golfe de Marseille oil il habite loujoura les fonds sableux ou sablo-raseux assez résistants; on le trouve à partir de $130 \mathrm{~m}$. de prof. et il descend jusquti it $6 \mathrm{~m}$, dans les fonds coralligèes au large de 


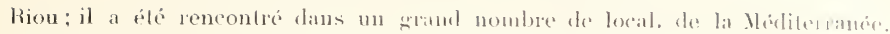
1) autre part, il existe sur nos coles ocedelentales de france, of dans certaines plages on le troure enfoner. tris superficiollement, dams le sable qui ne déconve

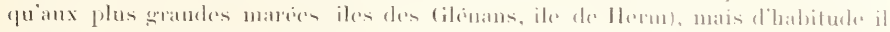

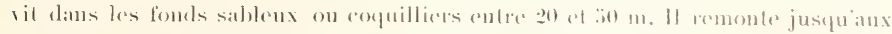

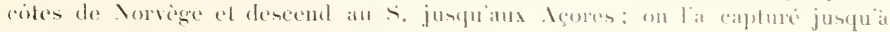
$900 \mathrm{~m}$. de prot.

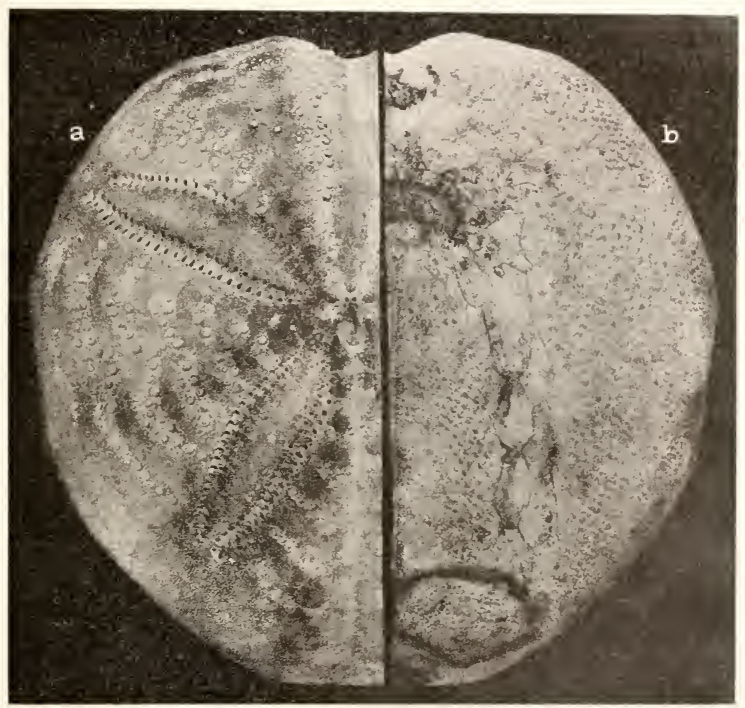

Fig. 90. - Spatangus purpureus; échantillon is corps élargi : a. face dorsale: b. face ventrale.

Montexsex, (1913, p. 2.), pt. 11, tig. 1 a dépit une var. caractérisée par des pétales pointus à l'exlrém., étroits ol conservant ì peu pris lat méme larg. sur la plus grande partic de leur long: aetlo lonme serail identique au sp. dieste-

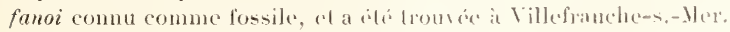

\section{BRISSOPSIS 1.. \.isisi\%}

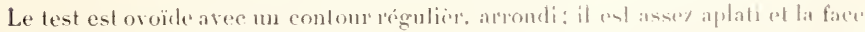

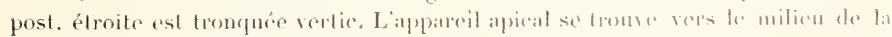

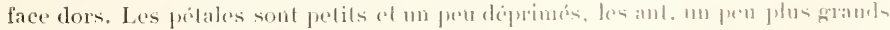

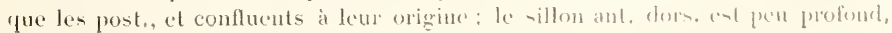


Le fasc. péripétale, qui entoure complet. les pétales a un contour sinueux et il est élargi au niveau des pétales ant. : lo fase. sons-anal est grand et large, un peu plus étroil en son milieu et situé en partic sur la lace ventrale: te péripr. ('n est tres eloigne. Les avenues ambul. ventr. sont larges et le plastron stemal est étroit.

B. lyrifera Forbes. Fig. 91. - Voir: Arissiz. 1×7?-187', p. 331, pl. IIX,

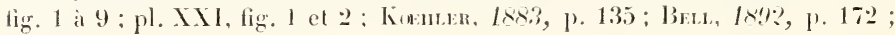
Mortexsen, 1907, p. 1.2.2.

L'espèce se reconnait très facilement it son fasc. péripétale dont la forme rappelle eelle d'une lyre el qui tranche netlement par sa conleur foncée sur le reste de la lace dors. converte te piq. gris. Le test, ovoüde et assez plat, est mn peu plus haut en arriere quen avant; sa long. atteint en movenne

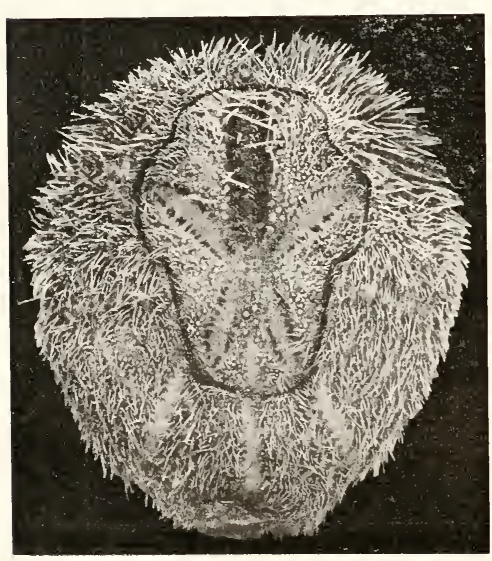

Fui. 91. - Brissopsis lyrifera; face dorsale diun échantillon muni de ses piquants: grandeurnaturelle. $50 \mathrm{~mm}$., la lark. $43 \mathrm{~mm}$. el la haut. 28 mm.; la long. peut arriver a 6.5 mm. Jes avemues ambul, rentr. sont presque nues. Le péripr. est ovale. plus long que large : le labre, court, atteint le milien de la première pl. ambul. voisine. Le fase. sous-anal renferme 3 paires de pores de chaque crité. Les piq. de la face dors. sont courts el pas très serrés, ceux du plastron sternal forment 2 toulles lat. ef sont souvent aplatis en spatules. Les pédic. sont de 4 sortes : tridact., rostrés, globil. et trif. Les tridact. ont les valves allongées et le limbe, élargi dans sa deuxième noitié, est finement denticulé; les rostrés ont les valves étroites; les globif. sont assez grands: leursvalves dépassent $1 \mathrm{~mm}$. de fong. ol oflrent une partie basibire tres large, landis que le lube est étroil: ils se terminent par : tres lomgues dents.

l.a couleur à l'étal vivant est d'un gris plus on moins fonci, ou gris verdatre.

La b. lyrifera se trouve dans des fonds sableux ou sablo-vasenx; nu Méditer-

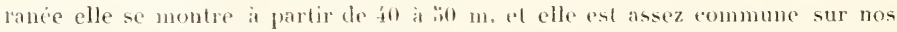
cotes de Provence: dans listlantique on la trome a partir de $30 \mathrm{~m}$. mais elle

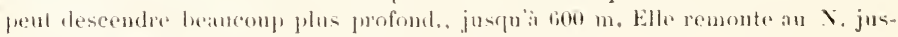

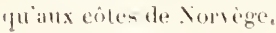




\section{G. BRISSUIS hLEIN,}

Le test est elliptique el allougé, l'appareil apical est reporté lrès en avant et

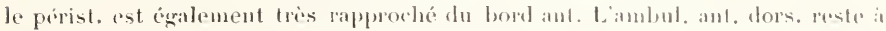

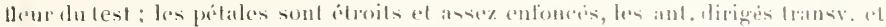

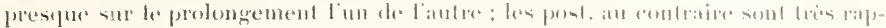

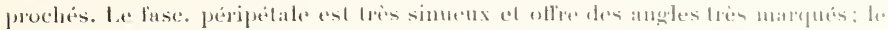

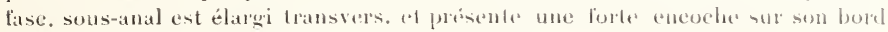
sup. qui est très rapprochí du péripr.

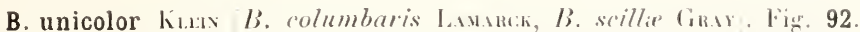

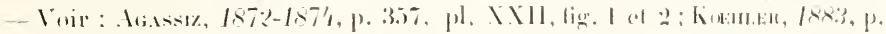

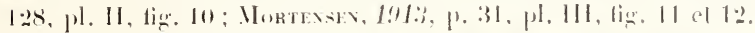

Le test est très grand et allongé; dans lindiv. que je représente ici, il atteint $100 \mathrm{~mm}$. de long., $8.3 \mathrm{~mm}$. de larg., 47 nom. de haut.: il exisle des indiv. encore plus grands. La face dors. séleve très rapidement jusqu'ì l'appareil apical, puis suit une courbe assez r'ég. jusqu'à la face post. qui est petite el oblique vers le bas: l'interrad. post. dors. est assez saillant, la face ventr. est un peu bombée: les avenues ambul. ventr. sont très étroites, tandis que le plastron stemal est très large. Le périst. est உ lois plus large que long, le labre est extrèmement courl é élarọi transvers. Le péripr., ovale, est presque 2 fois plus haul que large; le fasc. sous-anal

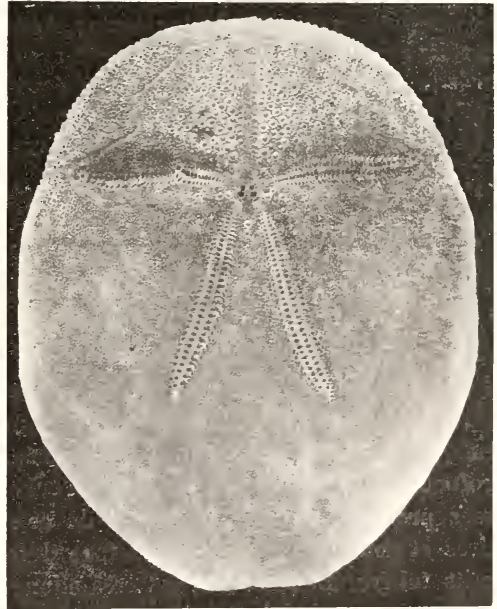

Fici. 92. - Brissus unicolor ; face dorsate, échantillon dinude, $\times 23$. renlerme 4 paires de pores de chaque crilé; le fasc. lat. forme en avant des pétales ant. mu angle rentrant aigu, et entre les pél. post. un autre angle rentrant beaucoup plus ourert. Les tuberc. de da face dors, sont gros, a peu près de mime taille que ceux de la face ventr. en avant des pétales ant., mais sur le reste de la lace thrs. iIs sont beaucoup plus petits: les piq. sout égal. très tius, serrés el soyenx sur cette partie de la face dors. 
En plu- de- í forme- lubit. de pédie. il existe des ophie. Les vatres des grandes pédic. tridact. présentent des dents très allongées, analogues à celles de l'Echinocardium penmatifidum fig. 13, a ; les globif. ont les valves courtes et ramassces, el l'ouverture terminale est munie de 4 à jo dents.

lat eonleur ì l'élal vivant est doun gris plus an moins foncé.

Le B. uniolor exinte sur nos cotes de Provence dans les fonds vasenx it une

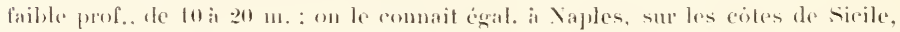

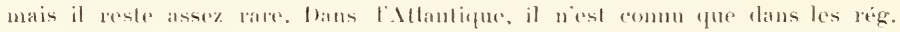
rbaudes Acores. Cimaries, iles du Calp Vert, Intilles), el il pent descendre jusqü 2 ì $\mathbf{t}^{\prime} \mathrm{m}$.

\section{G. EGHINOCARDIUM GiRAY.}

Le lest ast minee of plus ou moins cordiforme: en plus du fase. sous-anal, il existe un fance int., mais pas de fitse. prefipétale. Les pretales sont plus ou moins triangulates: la pertion de res pétales entermée dans le fasse. int. ne possède que des pores petits at plus ou moins obliterés. Le fase. soms-anal est ordin. anssi longr pur large, un peu corliforme arec une pointe inf. Le péripr. est situr sur la face post. du test yui est trongue vertic. La coulenr générale est grise. tantot gris clair, tantiot plus on moins foncée.

l. g. Echinocardium est representé sur nos côtes par ă espèces diflérentes.

to Espèces dont linuluul. ant. imparir est plus ou moins enfoncé :

E. cordatum Pexint $]$ [ I mphidelus r. Funbes $]$. Fig. 93. - Virir: Ag.ssiz,

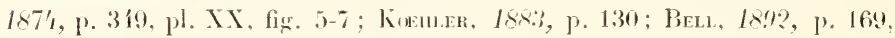

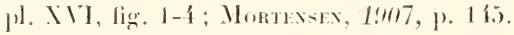

Le test est eordiforme. 11 pen plus lumgr que lange, ascez aplati : sa long. ne dépasse guere ó en. Vu par en haut, le conteur est un peu anguleux avec me forte échancrure ant. correspondaut au sillon dors. L'interrad. post. est rentlé eu une proéminence asse\% marquée. Le sillou dors. est très large et profond et il porte sur ses ciles des tuberc. prim. assez gros; le test est assez proéminent de chaque coité de ce sillon. Les pétales sont triangulaires el peu profonds. Toute la face dors. est uniformément couverte de petits tubere. Le fase. int. est très allongé ef tres large et il se prolonge beaucoup en avant; le fasc. sous-anat renferme 3 paires de pores de chaque coté. Le péripr., placé dans la partie sup). de la liace post. vertic. du test, varie beaucoup dans sa forme: sur les indir. de nos eotes il est général. allongé vertic. et plus haul que large, mais che\% d'antres il esl quelquefois ovalaire transvers. Le labre atteint la $2^{\text {me }}$ pl. ambul. voisine. Les piq. dors. sont très minces et soyenx, non dressés ; cenx de lia fiace ventr. sont plus forts et jus longs ct ils sont général: spatulés sur le plastron stemal.

Les pédie. sont de $\mathrm{f}$ sortes : tridart. stobil., rostrés el trif. Les tridact. ont les valves allongeos el géméral. minces ; les ghlobif. sont très apparents grâce surtout a leur eoloration pourpre foncée; leurs valves sont courtes el 
ramassées ; la partie basilaire est tres large et le limbe tubulaire, tres court. se termine par un orif. entouré de quelques dents.

L'E. cordatum est très répandu dans presque toutes les mer's. II est frequent sur nos côtes de l'Océan el de la Manche, et on peut le reneontrel dans le sable à mer basse. Il vit i) 15 ou 20 cm. de prof. dans une cavite tapissée par du mueus et qui communique aree le sol par 2 conduits; l'on trouve presque toujours avee lui 3 ou 4 Crustacés commensaux (Urothoe marina). A Wimereux, les pêcheurs appelent l'E. cordatnm " aruf de grisard". Sur nos còtes de la Méditćrannée, l'E. cordatum n'est pas très répandu, sauf rers les Bouches du lhone, Il vit général. à une faible prof., mais il peut descendere jusquä $1: 30 \mathrm{~m}$.

\section{E. mediterraneum For} Fig. 94. - Voir : Ag.ssiz, 1872-7', p. 580, pl. XXV, fig.

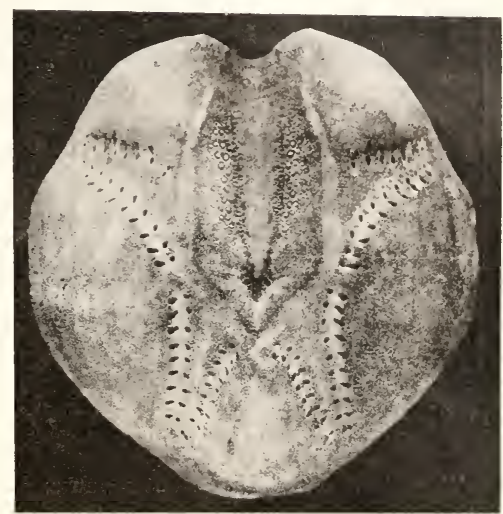

F1G. 93. - Echinocardium cordalum; face dorsale; légèrement grossi. 29; Kinhler, 1883, p. $132 \mathrm{el}$ 1898, p. 175, pl. IV, fig. 1-3; Mortexsex, 1907, p. 1.30.

Le test, à peu près aussi long que larwe, natteint jamais de grandes dimensions; les échant. ont $3,5 \mathrm{~cm}$. de long., exceptionn. í cur. Le contour vu d'en haut est anguleux avec une dépression ant. peu importante, el la rég. post. est rétrécie. Les faces dors. et ventr. sont aplaties et l'extrém. post. forme une gibbosilé asse\% marquée; les faces ant. el post. sont vertic. L: $E$. mediteraneum est surtout caractérisé par la forme du sillon ant. dors. qui n'existe qu'en avant du lasce int. et se trouve exatement localisé sur la face ant. vertic. dutest; ee sillon est d'ailleurs peu profond et étroit, of il offre sur son bord destubere à peine plus gros que les voisins. Le fasc int. est plutót court, puisqüil s’arrète au début du sillon ant. du test et il reste asse\% etroit; en dedans de ce fasc. se montrent plusieurs tubere assez développés. Le reste de la lace dors. est uniform. convert te petitstubere., mais ceux de la face ventr. sont plus développés. Le péripr. est allongé vertic.. étroit, 2 fois plus haut que large. Le fasc. sous-anal renferme une ou 2 p. de pores de chaque coté et le labre atteint la 2" pl. ambul, voisine. Les piq. de la face dors. sont fins, serrés, appliqués et mème feutrés; sur la face ventr., ils sont beaucoup plus gros, plus longs et'souvent recourbés: les piq. sternaux sont spatulés. Les pédic. sont de $\mathbf{f}$ sortes comme chez l' $E$. cordatum car il 
n'existe pas d'ophic.: les globif, onl les valves plus allongées que chez ce dernier.

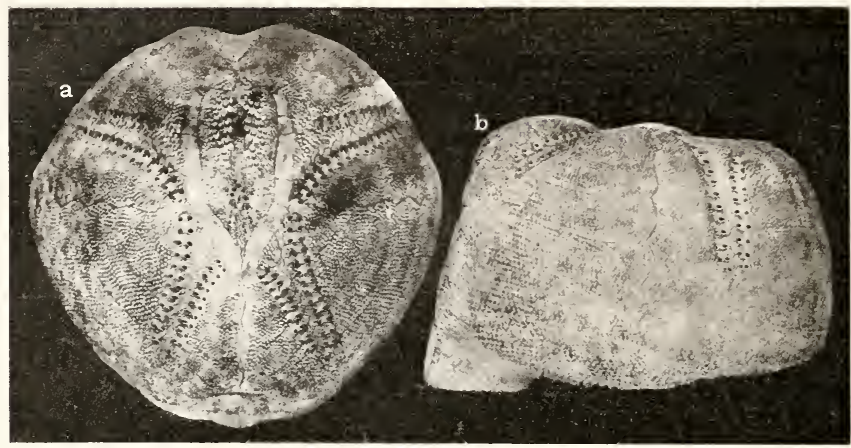

Fig. 94. Echinocardium mediterraneum. X 2; a, face dorsale; b, vue latérale.

L'E. mediterraneum os surtout eonnu en lléditerranéc oì il n'est d’ailleurs pas très commun. Je l'ai reneontré sur les plages de Foz (Bonches-du-Rhône) et de

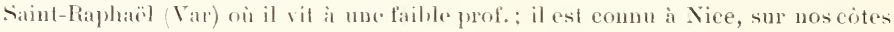

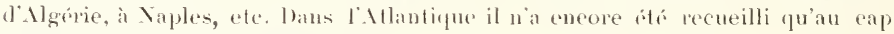
Sigres par la "Princessen Mlice ".

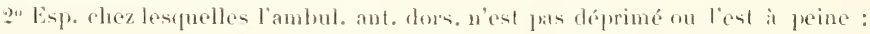

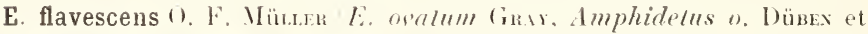
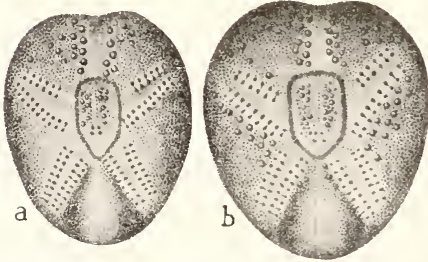

Fig. 95. - Echinocardium flarescens: lare dorsale, légèrement grossi ; a, échanlillon it laseiole interne allongri: b. echantillon ì fasciole élargi.

Lil: flusescens est esentiettement caractérisé par la presence, au milieu des tubere. tres fins qui reconvent toute sa face dors., de gros tubere qui se montrent surtout dans les aires interral. lat, ant. et le long de l'ambul. 
ant., et qui se retrouvent ausisi dans les 2 interrad. post., le long des pétales ant. Ces tuberc. servent à l'insertion de piq. beaucoup plus gros que les autres piq. de la face dors. qui sont très fins, feutrés et serrés. Le fase. int. est petit, court et étroit. Le fasc. sous-anal renlerme 1 ou 2 paires de pores de chaque coté. Je périst. est assez grand, pas beancoup plus large que

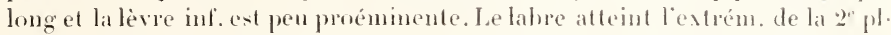
ambul. voisine. Le péripr. est relativ. gramd, a pen prés ansi hong que large.

En plus des í formes habit. de pédic. les jemes posident desophic. Les globif. offrent un tube étroit qui se termine par ti it s dents tris allongées (fig. 99, b) ; la forme des pédie tridact. est caractéristique el lem limbe est très élargri fig. 99, a .

La conlemr a l'état vivant est d'un hlanc grisâtre, quelquefois lépèr. rosée.

En Méditerranée, l'E. flaresceus se troure sur nos cótes de Provence it des prot.

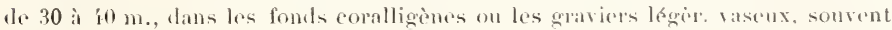
associe an Spatangus purpureus of an Schizaster cunaliferus. It it ingatement dans l'Atlantique el partois at me tris fithle prof. 6-8 m. a Concarneam, main il sétend jusqu’à $150 \mathrm{~m}$. Il remonte justuraux coles de Vorrige at d'Islande, et peut descendere justuatax Acores.

E. mortenseni Tumn E. intermedium Mortrisco . Fig. 96 el 98. -

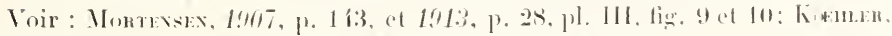
1909, p. 2 fo, pl. ХХХ, fig. 2-6 E. intermedium.

Cette espèce arait d’abord été considérée comme nu E. furescens alteignant une plus gramble taille que d'habitude : il présente en eflet le même contour rég. et la même forme ovalaire que ce dernier. Lu échant. que jai recueilli a Toulon mesure $511 \mathrm{~mm}$, de long, f.s mm. de largog. te 32 mum, de haut. Indépendanment de sa taille beaucoup plus wrande, il sécarte de l'E. flasescens par l'abanduce de grostubere., et par suite de gros pin.. sur la fase dor. dans be interrarl. lat.: le revotement des piq. de lia latee dorse du test est uniforme ef cest a peime sit existe quelques piq. un peu plus grands vers le bord ant. du test. De pluss. les pédic. tridact. ont une forme bien differente : les valves sont assez étroites an lieu d'etre élargies comme chez l'E. flureseens

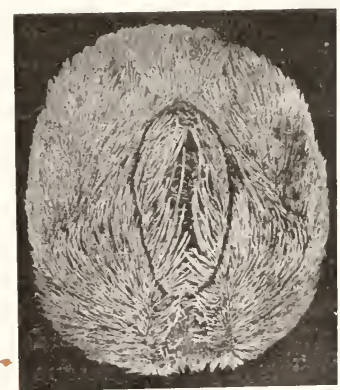

Fu. 96. Echinocardium mortensemi: animal muni de ses piguants: grandem naturelle. fig. 98. Le lase. sous-anal renferme 3 paires de pores de chaque coté, nombre sup. à celui que l'on observe chez. l'E. flasescens. le labre alteint l'extrém. de la $2^{\circ} \mathrm{pl}$. ambul, voisine. 
L'E. mortenseni n’a encore ólé rencontré que dans la Méditerranée et seulement daus 2 localités : à Toulon, où des pếcheurs l'ont capturé à une prof. de $10 \mathrm{~m}$., et is Naples où il vit entre 1 't et $40 \mathrm{~m}$.

On le distinguera facilement de l'E. flavescens à sa taille plus grande, à l'absence de grands piq. ou de gros tubere. sur les aires interrad. dors., et à la forme des pédic. tridact.

E. pennatifidum Norm.x" Amphidelus gibbosus B.ırett. Fig. 97 et 100.

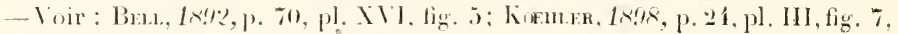
pl. IV, lig. 9-11; Montexsex, 1917, p. 139.

C'est le plus grand Echinocardium de nos mers car, sa long. peut

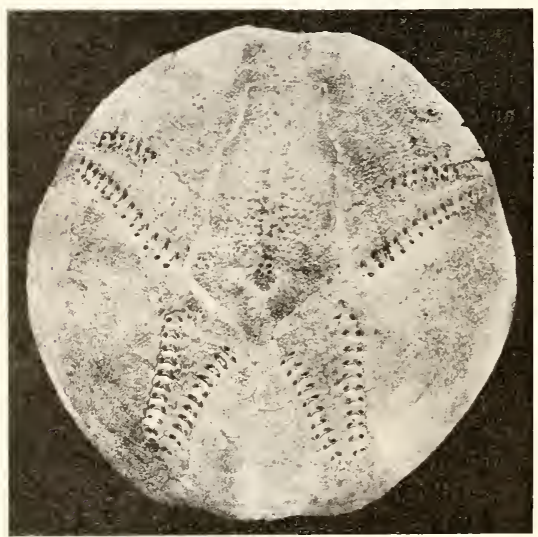

Fis. 97. - Echinocardium pennatifidum; face dorsale: grandemr naturelle. atteindre $7 \mathrm{~cm}$. Dans l'échant. que je représente ici, la long. est de $62 \mathrm{~mm}$., la larg. de $60 \mathrm{~mm}$, et la haut. de fil mm.; certains indiv. sont légèr. plus larges que longs. Le contour du test vu d'en haut est régulièr. ovalaire, presque circulaire, avec une légère troncalure en avant et en arrière. Vu de profil, le test suit une courbe très régul. jusqu'au niveau de la face post. qui est tronquée vertic.. L'ambul. ant. est à peine déprimé ¿l lambitus. Le fase. int. est très allongé : ses deux branches ne sont pas exactement parallèles et il est plus étroit en avant qu'au niveau de l'appareil apical :

il renferme destubere. assez gros, mais lout le reste de la face dors. est uniformément couvert de petits tiberc. Les pétales sont à fleur du test. la face rentr. est converte de gros luberc. Le fasc. sous-anal ne renferme que 2 paires de pores dechaque côté. Le labre est extrêm. court et il n’alteint que le milien de la première pl. ambul. voisine; le péripr. ext élargi transvers.

Les pédic tridact. se présentent sous 2 formes: dans l'une, très grande, la tele peut atteindre $2,5 \mathrm{~mm}$. de longueur et les valves sont munes, sur les lopds, de dente peu nombrenses mais trés longues et tres développées, dispositim qui a fait domner son nom a l'esp. fig. 100, b : l'autre forme a des denticulations plus lines. Les valves des pédic. globif. offrent une partie basilaire extrem. développée, très large el un tube très court dont l'ouverture termin. porte $i$ i 5 dents de chaque coté ligr. 100, a Les rostrés ont 
en général les valres linement denticulées mais cerlams ullumb des denls longues el ćpaisses, peu nombreuses $\mathrm{c}$ ), laisant ansi passige aus tridactyles.

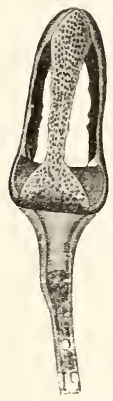

Fiti. 98. E Echinocardium mortenseni: pédicellaire tridacyyle, $X$ :

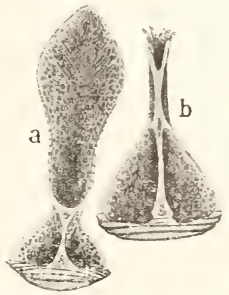

Fis. 99. - Echinocamlinm flucescens: a, valve de predicellaire Iridactyle, X $2: \mathbf{b}$. valve de pérlicellarie globilere, $X$ :

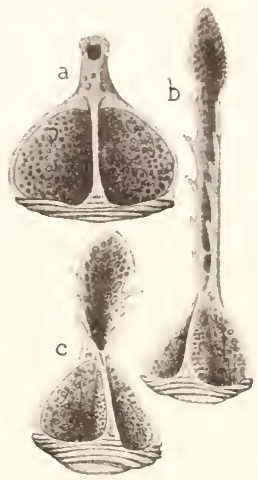

Fu. 100. - Eshinocardium pelmutifitum: valves de pedicellaires: a pédiceldaine globiliere, $X$ 2:i: b, pédicellaire triclaclyle allongé, $\times 3.8$ : c, pédicellaire rostré. $\times$ :

L'E. pennatifulum rappelle par divers caract. les E. flarescens al intermedium. 11 dillère du premier pret sa grande latille, par son fase, int. tros allongé, pau l'absence

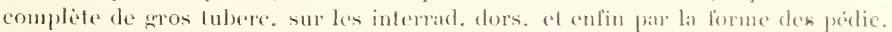
tridact. el globif. Il est plus voisin de l'E. mortenseni qui ast plus grand que l'E: flacescens et l'on pourrail parfois hesiler entre des E. mortenseni adultes ot des E. pennatifidum jeunes. On dislinguera ce dernier is son linse. int. plus allongé, it son labre nalleignant pas le berd post. de la premiere ple ambul., tamdis gu' il

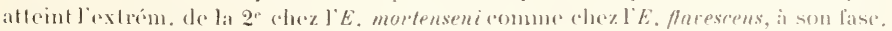

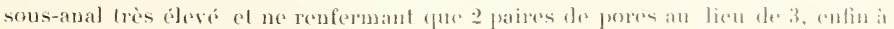
la forme bien diflerente des pedice triblacl. el globit.

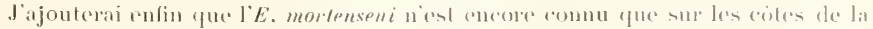

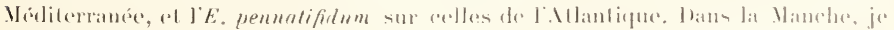

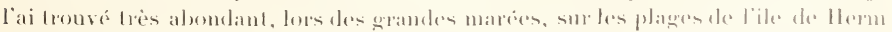

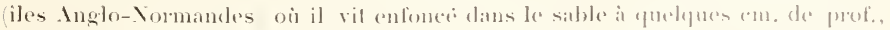

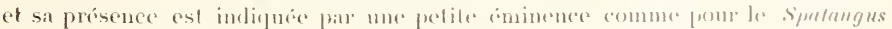

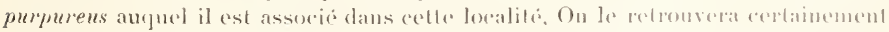

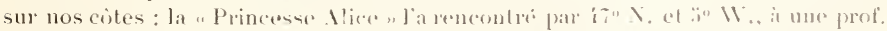

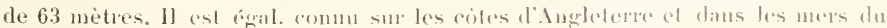

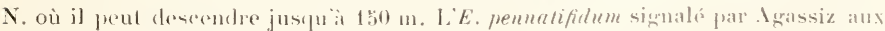
États-Lnis est une: antre espece. 


\section{HOLO'THURIDES}

Une Holothurie ne peut ètre mieux comparée qu’à un gros Ver cylindrique pouvant atteindre et mème dépasser $30 \mathrm{~cm}$. de long. La bouche, entourée d'un cercle de lentac., se trouve à l'une des extrém. du crlindre. Pour comprendre l'organisation de l'Holothurie, on peut supposer un Oursin dont le squel. serail réduit à de très petites pl. isolées, ef quon aurail étiré de manière à convertir son corps sphérique en un corps crlindrique fig. 103. Les rad. et les interrad., au lieu de correspondre aux méridiens d'une sphère, seraient donc placés suivant 10 génératrices du cylindre. Les appendices ambul. sont constitués par des lubes allongés, ordin. rétractiles et munis d'une vent. term., ou par des papilles dépourvues de vent. Les tubes ambul. ou pédicelles sont souvent localisés sur un des côtés du corps formant une face ventr. (qui correspond au irivium sur laquelle rampe l'Holothurie. la bouche en avant. Pour la placer dans la même position que l'Oursin auquel on la compare, il faut donc la redresser vertic. et la placer la bouche en bas fig. 1 .

Les parois du corps renferment des glandes à mucus et surtout des corpuscules calcaires ou sclériles isolés, très petits, représentant le stade jeune des pl. de l'Oursin et dont les lormes, très caractéristiques, sont importantes pour la clasification et la détermination. Cessclér. se montrent sous formes de pl. perlorées, tantit réz. el strmétriques, lisses ou munies d’aspérités, de tubere. ete; de bitonnets droits ou arqués, simples ou ramifiés, pleins ou perforés ; de corpuscules crépus dont les ramit. courtes, subégales et serrées, ont l'extrém. arrondie et forment un ensemble sphérique ou ovoïde; de corbeilles grillagées formées de travées très minces, recourbées et réunies par un cercle ext.; de corpuscules turriformes ainsi nommés parce que d'un disque basilaire s’élèvent descolonnettes général. au nomb. de 4, quisont rémies par des travées transv. et dont l'ensemble a été comparé à une tour. Les pl. qui ont une forme très rég., elliptique, avec quelques perforations symétriques, sappellent des boucles. On trouve ordin. chez la mème espèce plusieurs lormes de sclér. et les corpuscules superficiels des lég. sont souvent diflérents des corpuscules profonds. Dans certaines espèces, les sclér. sont très réduits comme taille et comme nombre, p. ex. chez l'Holothuria forstials de nos cotes.

Je serai très bref en ce qui concerne l'organisation inl, des Ilolothuries et 
ne rappellerai que les dispositions utilisées dans la clascifieation. Te tube dig. lig. 8, D part de la bouche et se dirige vers lanus en se rapplorehant

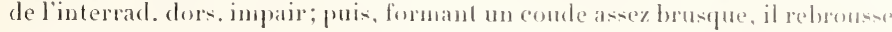
chemin vers la bouche en suivant l'interad. dors. ganche, mais avant de

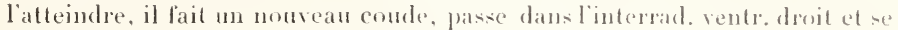
dirige de nouveau vers l'anus pour souvrir au dehors; des mésenteres rattachent à la paroi du corps ces 3 segments du lube dig. Dans certaines Synaptes, son trajet devient presque droil. Lal régiom pharrngienne est entourée de pièes calcaires formant lannenu pharyngien, et qui ront an nomb. de dix : 5 rad, et 5interrad. Lappareil aquifere comprend un cercle oral duquel partent 5 branches rad. formant des canaus longrit. qui fournissent des ramifications aux appendices ambul., plus 10 canaux tentaculares qui aboutisent aux tentac. $\mathbf{T}$. An cercle aquifere sont annexées une ou plusieurs vésicules de Poli P parfois très grandes et atteignant un ou $2 \mathrm{~cm}$. de long.. plus un tube hydrophore ou canal du sable sc , qui souvait au dehors chez la larve, mais qui, chez ladulte, sourre simplement dans la carité générale, excepté dans un groupe d'Holothuries abrssales; il peut aussi y aroir plusieurs tubes hydrophores.

On dérigne sous le nom d'organes arborescents, et improprement sous le nom de poumons, 2 org. très développés consistant en ranifications très nombreuses qui se réunissent en 2 trones volumineux Wl šinsérant sur la partie terminale de l'intestin cloaque). Ces organes peuvent se remplir d'eau qui pénétre par l'anus et sert à la respiration : Iorsquils sont complèt. remplis, le corps tout entier de l'Holothurie est gonflé et turgescent; lorsqu'au contraire l'eau qu ils contenaient est expulsée par l'anus, le corps devient flasque et mou. Sur la paroi du rectum s"inserent parfois des tubes particuliers dont l'ensemble constitue l'organe de C'uvier. Ces tubes, dont te nombre varie de dix à une centaine, forment un faisceau très serré et ils s'insèrent chacun isolément sur le rectum : ils sont tris fins, cylindriques, allongés, de coloration blanche ou jaune brunatre. Sous l'influence d'une excitation, p. ex. lorsquion prend l'Holothurie a la main, on la voit rejeter par l'anus un certain nombre de ces tubes qui sortent très rapidement, poussés avec une grande force par les contractions de l'animal; en arrivant dans l'eau, les tubes de Cuvier subissent un gonflement considérable : ils deviennent très longs en même temps que leur surf. devient glutineuse et visqueuse, de telle sorte qu'ils adhèrent très fortement à tous les corps étrangers el peurent emprisomner de petits innmaus. C'est par la déchirure du cloaque que les tubes de Cuvier sont ansi exputsés. Hs manquent d'aitleurs chez de nombreuses Holothuries ot n'existent que chez les Aspidochirotes, principal. dans le g. Holothuria. Leur présence chez certaines espèceconstitue un caractère taxonomique d'une tres grande valeur et il serait logique de classer dans un g. ̀̀ part les espèces d'Holothuries qui les possèdent.

Les oryanes genit. $\mathrm{OV}$ consistent en lubes simples nu ramilies formant 


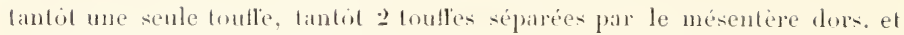
débouchant dans mon canal gui somve au dehors entre les tentac. dors. Les

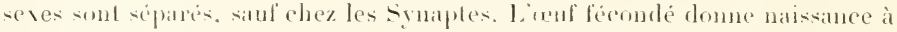

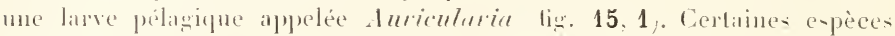

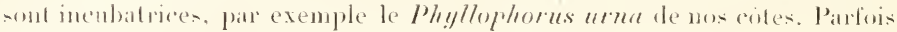
Jlobothurie peut se reprodure par simple division transr. : le fait, fres. rare, dे é丶 signalé che\% les Curumatia lactea el planci de mos cotes.

Les Ilolothuries vivent en mer, depuis le niveau des marées basses jusquaus plus grandes prof. En Méditerranée, on pent voir, à 22 ou 3 m. de prof. el meme moins, les grosses Ilolothuria, II. Lubulosa, II. polii, el

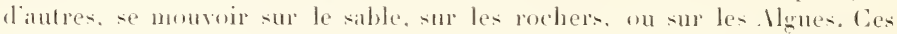
IIolothuries rampent sur lenr face ventr. an moren de leurs lubes ambul.

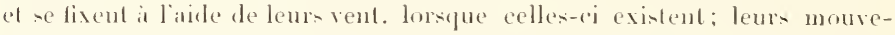
ments sont d'ailleurs tres lents, et beancomp de formes, notamment les C'acumatia, peuvent rester à peu pres immobiles pendant fort longtemps. Jen tentac. servent a l'llobothurie à eapturer les particules alimentaires qui comsistent en petits anmaux, débris d'organismes morts, ele.; me fois que ces debris sumt saisis par un tentac, celui-ci se rétracte, se recourbe et pousse lat particule alimentaire dams la bouclue.

Lorsquion saisil a la main une llolothurie, celle-ci se rétracle plus ou moins rapidement en expulsant par l'anus me certaine quantite du liquide contenu dans les oromes arborescents: les tentac. et les appendices ambul. se contractent auss rapidement. A la suite de ces contractions, le tube digestif est souvent rejeté par le cloaque : dans le gr. Ilolothuria, el notamment ches l'II. tubulosa, il est rejeté tout entier par l'anus: ce rejel, très violent, est très rapide, el le lube dig.. qui entraine avee lui l'oro. arboresent droil, brise, pour sortir, les parois du cloaque: dams le g. Thyone, les deux org. arborescents et mème les org. wénil. sont rejetés. Chez les especes qui possedent un org. de Cuvier, les fubes de celui-ei sont expulsés aviut l'appareil dig. qui ne sort que quelque temps après. (In a comstaté dans certains eas que le tube digr. expulsé pourail êre régénéré.

La détermination des llolothmries est beancoup plus diflicile que celles des autres f́chinolemes : il ext peu de groupes che\% lesquels les erreurs de délermination se soient montrées ausi nombreuses, et chez lesquels anssi la sronomie soit aussi riche. Jes caracteres ext. fommissent, à l'état vivant des indications qui sont surtont utiles chez les Ispidochirotes, et le zoologiste exercé recommatra de suite a première vue lat pluparl de nos especes des g. Molothutia et Stichopus; chez les Jendrochirotes et les symaptes. les caracteres ext, ont moins de valeur. Dans fous les cas, el surtoul si l'ous at

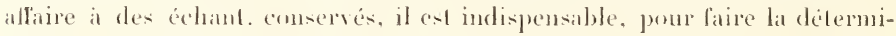
nation, d'abord de compter lestentac. puis d'ouvir l'animal pour examiner les ors. int.. et enfin d'étudier les sclér. Le nombre des lentac. permetlra de

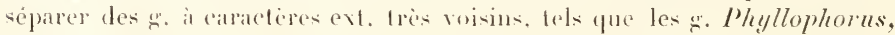

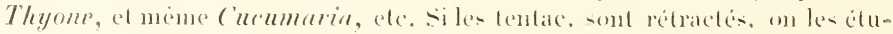


diera en ourant le pharyns. Lieximen des orgo. int. permettra de reconmaitre la forme des pièces calcaires de lamneau pharvngien, le nomblue de vésic. de Poli el des tubes hydrophores. le mombre, le developpentent al lat

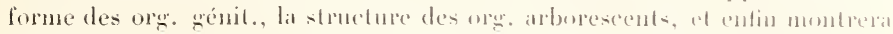

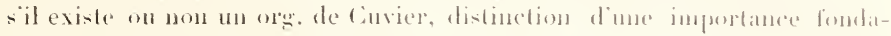

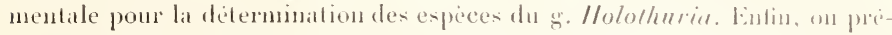
levera de petits fragnents des tign., des appendices divers du corpe el der

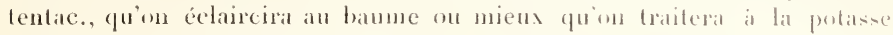
boullante pour éludier les sclér.

Est-il besoin de fare remarquer que la dedermination des Hotothuries ne

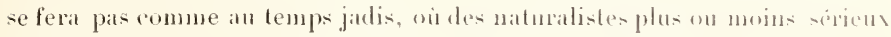

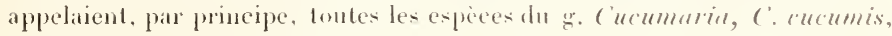
sous prétexte quelles ressemblaient a des cornichoms, on domaticul te mon d'Il. tubulosa ì toutes les especes dug. Ilolothuria provenant de nuscotes... et i beancoup d'autres:

La conservation des Ilolothuries demande quelques procautions, el lion peut parlaitement obtenir des échant. bien élales. avant une apprarence voisine de celle de lanimal vivant, au lien de ces choses informes quin voit si sourent dans les collections. J’ai déja donné p. 12z la technique à employer.

\section{TABLEAU DES ESPËCES}

1. En plus des tentac. péribuce, il existe des append. ambul., principal. sur les rad., et se présentant, soit sous forme de lubes ou pédicelles érectiles terminées par une vent. et servant à la licomotion, soit sous forme de papilles coniques S. CI. HOLOTHU-

\section{RIES PÉDIF ÈRES}

- Les seuls append. sont les tentac. péribuce. et la surf. du corpsest complèt, nue, les pédicelles et les papilles laisant détaut s. Cil.

\section{HOLOTHURIES APODES}

2. Les tentac. sont arborescents, c'est it dire que des ramifications de 2 ordre naissent à différents niveaux sur me branche principale, puis se divisent à leur lom en ramitications de $33^{\prime \prime}$ stretre, etc. O. DENDROCHIROTE

- Les tentac. sont peltés. c. a d. que leurs ranitications partent exclusivement de l'extrém.; ils olfrent une tige simple cl les ramilications, assez courtes, arrivant foutes an mene nivean, forment dams leur ensemble me sorte de disque épais (1. ISPLDOCIIIROTES

3. Tentac. au nombre de 10, dont ? phos petits, qui occupent toujours une situation ventr. 
- Tentac. en nombre sup. a 10, inegans el les plus petits en nombresul). à 2 .

' rangés rad, bien distinctes, entre lesquelles peuvent se montrer quelques lubes interrad. plus petits et plus ou moins abondants. Pas de dents anales G. C'ucumaria

- Les tubes ambul. sont répartis irrégulies. sur tonte la surf. du corps sans former de rangeses rad. plus particulierement nettes: des dents anales (i. Thyone.

5. Append aubul. constitués par des tubes réguljèr. disposés en 2a rangées sur la face ventr., et par des papilles moins serrés et irrégu-. lièr. disposées sur la face dors. Tubes génit. terminés par une extrém. rentlée, piriforme et aplatie. Les sclér. ont comme forme de départ un corpuscule en huette avec 으 orif., ou en losange avee torif: de cette forme fondanentale dérivent des sclér. plus compliqués, et, chez les jeunes, il existe de grandes pl. éparses avec de nombreuses perfor. : corbeilles petites et incomplètes

C'ucumaria montagui p. 1.50

- Les append. ambul. ollrent la mème forme dans les a rad.

i. Certaines pl. destég. sont très épaisses et ont la forme de cones de Sapin avec un réseau calcaire très dense. devenant plus délicat vers l'extrém. la plus étroite.

- Pas de corpuscules en forme de cones de sapin.

7. Lestubes ambul. forment plus de gangés dans la plupart des rad. et manquent dans les interrad. Les pl. epaisses en cones de Sapin, sont accompanées de pl. plus pelites a hords denticulés, munies le plus sourent de 2 ou 1 perfor. trés élroiles qui font mène lefant parfois . . . . . . . C'grubei P. 15 i

- Les tubes ambul. ne forment que z rangees dam les rad. et existent anssi dans les interrad.; les corpuse. en crines de Sapin sont accompagnes de pl. ovalaires munies de gros nodules arrondis et de perforations. . . . . . . C. syracusana p. 15.)

8. Les tubes ambul. sont rigides et nou rétractiles: lestég., rigides eux-mèmes, renferment de grosses pl, perf. dont l'ensemble constilue une sorte de cuirasse résistante

- Les tubes ambul. sont rétractiles. les tég. renferment des pl. plus (u moin- décloppées mais ils ne sont pas rigides.

e corps a la forme d'un croissant plus on moims recourbé, épaisis en son milien et aninci anx extrém. les tubes ambul. sont gros, coniques, pas trís serres, et disposés en zig-zag plutit qu'en deux rangées distinctes: les pl. sont particul. grandes et allongées . . . . . . . . . C. lergestina p. 158

- Les tubes ambul.. asse\% nombreux et serrés, sont petils, courts. el disposés en 2rangers. 
11. Le corps ent allongé, droit on quelque pen recourbe; lat rigion post. est très anincie sur une asse\% grande long. of forme une sorte de quene très apparente. Lespl. sunt tresgrandes: les cort. portent sur leur cercle ext. des dent - an de- lole-plus ou mons nombreus et bien ipparents. . . . . . Cucumaria elongata p. 160

- Le corps est plus on moins fortenent recourbe. lextrén. post. est raceourcie el identique à l'estrem. ant. ; les pl. sont de movenues dim.; le cercle des corb. ollre un bord. ext. lisse on muni de petits lobes peu développés. . . . Cucumaria cucumis p. 161

11. Especes de tres petite taille pasplus de 2 em. de long. avee des tubes ambul. peu nombreux el disposés irrégulièr. sur une seule rangée ou stuivant une ligne en zig-zag . . . . . . . . . . . . . 1

- Especes de taille moyenne ou assez grande dont les tubes ambul. rad. sont disposés sur 2 rangées distinctes. . . . . . . . . 1)

1?. Couleur général. blanche. Les pl. des tégr. sont munies pour la plupart de nodules arrondis, et elles ont pour point de départ une pl. losangique avee 2 pertor. princip. el 2 perfor. plus petites placées perpendicul. aux précédentes. Il existe, en ontre, dins la couche superficielle, de petites corbeilles dont les travées centr. sont général. disposeses en crois ef fournisent de petites loranclues périphériques non ramiliees. . . Cucumaria las'lea p. 163

Conleur brume. Les pl. des tég., ovalaires et munies de modules arrondis, wlient des mailles plus éparisses el des orif. plus petitel moins régulièr. disposés que dans l’espèce précédente. Lescorb. sont formées de travees plus nombreuses et leurs ramilications se réunissent souvent de manière à limiter de petit-orif. périplrér.

Cucumaria brumnea P. lir.

13. Les sclér. sont formes surtout de pl. épaises, a surl' irrégulièr. mamelomnée et à perfor. nombreu-es disposées en ramgeres paral-

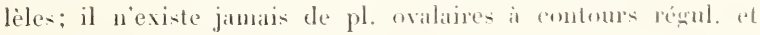
munes de nodules arrondis placés smétriquenent aver des oril. eux-mèmes symétriques; les corpuse. destubes ambul amsintent essentiellement en batomuets arqués du milieu desquels sálievent 2 petites tiges convergentes, formant une fourelle rudimentare. Pas de corl).

- Les sclér. comprenuent surtout des pl. ovalaires. munies de grom modulessphériquéstous égaux, dispu-és trés régulièr. el trèn symétriquement, avec desoril. placés égalicr. d'une manièresmétrique. 15

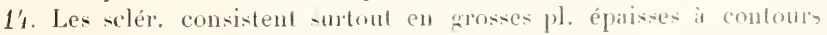

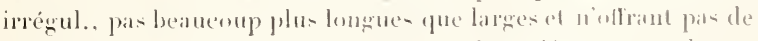

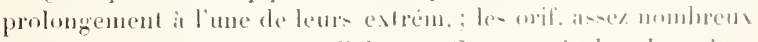

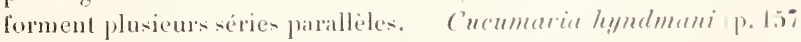

- Les pl. princip., plus longues que larges. noflrent ardin. que 2

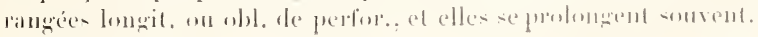


i linne de lenrs extrem. qui ent plus amincie, en une pointe lisse

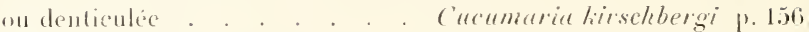

1.j. Outre les pl. walatires, de lomestmétrique el munies de molules, il en existe d'antres plus grandes, allongées. offrant is liune de

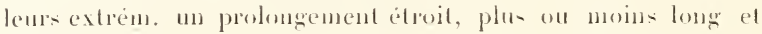
mumi de quelques pelites puintes lat.; il existe des predicelles interrad.

* Cucumaria liobllikeri p. listi

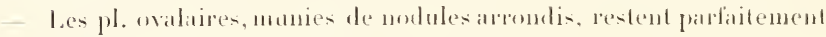

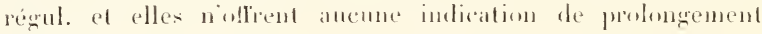
terminal.

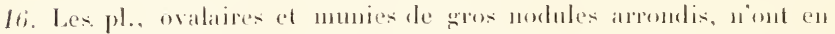

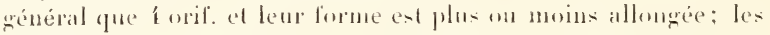
corbeilles, grandes, soul constiluées par des batzuettes épaisses el fortes; tég. plissés: des pédicelles interrad.

C"ucumaria lejerrei p. 152

- Les pl. "valaires on eylindriques, mumies de gros lubere., ont m nombre d'oril. sup. a i : corbeilles petites el délicates: lexw. lisses: pas de pedicelles dams les interrad. C Cucumaria planci p. 153

17. Les pédicelles sont répartis sur toute lis surl. du corps sims dilférence entre les rad. el les interrad. : les tentac. ext., au nombre d'une dizaine el tres qrands, lornent un prenier cercle entonrant un cercle int. d'une demi-douzane de tentac. plus petits: les sclér. consistent surtout en corpusc. crépus, très nombreux el en pl. pertorées de lorme irrégul., arec, en plus, quelques petits corpusc. Lurrilormes . . . . . . Phyllophorus urna p. 16!9)

- Tentac. au nombre de 18 a a 20 , en 2 cercles, les 10 ext plus grands. les 8 i $10 \mathrm{int}$. plus petits. Les pédicelles n’existent que sur les rad., au moins dans les régions ant. el post. mais ils peurent former plus de 2 rangées et vers le milieu du corps, il peut exister quelques pédicelles interad.; les sclér. consistent en corpusc. lurriformes très grands dans l'espece principale de nos cotes; pas de corpuse, crépus G. Pseudocucumis . . . . . . . . . . Is

18. E-pece de grande taille atteignant 15 a $20 \mathrm{~cm}$. de long. ; les corpusc. lumilormes ont le di-que qrand et la tourelle très développée. formée de í colonnettes: un cerlain nombre de pédicelles dans les intermal. . . . . . . . Pseudocucumis mixta p. 168 ispece de trexpelite taille (b a 10 mun. de long.). Les corpusc. turrilormes ont le dispue basilaire irregulier. arrondi avee plusiens.s perfor. et les tourelles peu développées consistent seulement en z colonnetter obl. Pas d'apjend. interrad.

Pseudocucumis marioni( (p. 16:)

19. Les tég. sont déponrrus de selér, ed cenx-ci n’existent qu’an roisinage de lamus el dans les pédicelles. . . . . . . . . ?t

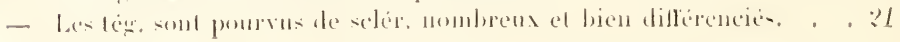


20. Les tubes ambul. noffreut quin disque term. sauf les 10 tubes qui bordent l'anus et qui renferment quelques bitonnets simples ou ramifiès. Les tég. soul gris-rosé, ou brun-rosé, opaques el assez épais. . . . . . . . Thyone roscosiln p.lfiti

- Les tég. de l'extrém. pont. du corps renferment quelques corpusc. turriformes dont le- disques arrondis sont parfois incomplets, les tourelles elles-memes sont tantit bien formées, tantôt incomplétes. Les pédicelles ne renferment qu'un disque term. Tég. rosés ou rouges, transparents . . . . Thyone inermis p. 167,

?1. Le corps, plutot court et recourbé sur lui-mème, oflre une rég. ant. assez élargie tandis que la rég. post., fortement amincie, constitue une sorte de queue, le tout atteignant $30 u \mathrm{fm}$. de long. au maximum. Les tég. sont remplis de pl. assez grandes. aplaties et lisses, tle forme irrégulièr. arrondie; il existe en plıs de petits corpusc. crépus . . . . Thyone raphanus p. 16.j

- Le corps, cylindrique el allongé, non recourbé, peut atteindre une lomg. de 10 à $15 \mathrm{~cm}$. sur 2 à $3 \mathrm{~cm}$. de larg. Les sclér. consistent principal. en corpusc. turriformes dont le disque, allongé et élargi en son milieu, offre le plus souvent 4 perfor. et porte 2 petites colonnettes courtes qui sont réunies par une anastomose transiers.

- Tubes génit. divisés en 2 faisceaux de chaque cóté du mésentère dore. G. Stichopus.

2. Tubes génit. groupés en faiscean unique $\mathbf{G}$. Holothuria .

23. Corps cylindrique; couleur ronge i l'état vivant: sclér. formés de corpusc. turriformes et de bitonnets aplatis et élargis, ordin. divisés ou ramifiés, en forme de croix, etc. Stichopus tremulus p. $\mid$ s|

- Corps aplati: conleur brune ou d'un brun-rosé à l'état virant avec des taches blanches: sclér. formes surtout de corpusc, turriformes et de bâtomnets non ramifiés. . Stichopus regulis p. 18.2

2'،. Tous les append. ambul. sont des papilles ayant la méme forme sur la face dors. que sur la face ventr.: les sclèr. consistent en corpusc. turriformes bien développés dont le disque est circulaire avec les bords lisses, et en boucles ovalaires à 6 orif.; un org. de Cuvier. .

- Les append. ambul. ont une forme différente sur la face dors. et sur la face ventr., cette dernière portant des pédicelles et la première des papilles

25. I.es sclér. des tég. et des pédicelles ventr. sont extrèm. petits, très rares et réduits a des pl. rudim. oflrant f orif. smétriques et égaux: seuls les tentac. posédent des hatomets. I.es téz. trèmous sont général, de couleur foncée, souvent uoire et le- papilles dors. ont ordin. l'extrém, blanche: un ory, de Cuvier trés développé llolothuria forsticeli (p. li!), 
les sclér. de- tég. nombreux el trè développés, consistent principal. en corpuse. tumitomes et en boucles ovalaires: des batonnels dims les pidicelles el les tentac.

$\because 6$. Lescorpusc t urriformes, de grande taille, ont un di-rque arrondiaver: des bords lines el des orif. régulier. el sumétriquement disposés..

- Les corpusc. turriformes peu abondants, sont tres petits el leur disque, dont les perfor. sont peu nombreuses, est muni sur les bords de pointen fortes et irrégulièr. di-pories; les selér. sont surtout constitués par des boucles

:7. Espèce de petite taille. Les pédicelles forment sur les 5 rad, des rangées distinctes. entre lesquelles se montrent des pédicelles interrad. plus rares. Les corpusc. turriformes sont bien développés et leurs tourelles, longues et minces, ont plusieurs étages.

* IIolothuria helleri p. 180)

- Espèce de grandes dimensions. Les tubes ambul. n'existent que sur la face ventr. ou ils sont trés serrés el souvent disposés en 3 séries plus ou moins distinctes; la face dors. est couverte de papilles très grandes, allongées, coniques el serrées; les corpusc. turriformes ont un disque à bord ondulé mais lisse et une tourelle large, épaisse el assez courte. Holothuria sanctori p. 171

28. La face dors, est munie de papilles coniques et pointues, de dimensions variables, plus ou moins nombreuses et pas très serrées; les tubes ventr. sont serrés et nombreux; pas d'org. de Curier. Espèces de grandes dimensions (jusquà $30 \mathrm{~cm}$.

a face dors. se fait remarquer par des éminences en forme de fros mamelons très volumineux, souvent disposés en rangées longit. assez distinctes; les pédicelles ventr. sont peu serrés; un org. de Cuvier. Les exempl. connus ne sont pas lrès grands 12 a $13 \mathrm{~cm}$. . . . . . . " Holothuria mammata (p. 17 .

?!. Lextrém. des append, ambul., pédicelles ventr. et papilles dors., offre une couleur blanche qui tranche nettement sur la coloration générale trés foncée, violetle ou noire, des tég. ; la -urf. des boucles est lisse.

llolothuria polii p. 1 is

- La conleur générale est brune, plus claire sur la face ventr. plus loncée sur la face dors.; la surface des boucles est plus ou moins rugrueuse el garnie de pelites aspérités pointues.

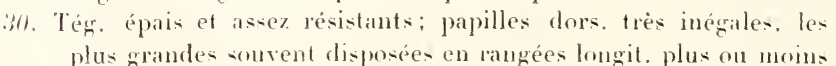
distinctes; les boucles possèdent en général 3 paires d'oril., mais elles peuvent s'allonger considérablement et en acquérir 12 a 15 paires, parfois ces orif. sont eomplèt, obturés.

Holothuria tubulosa p. 17 í.

- Tég. assez minces; les papilles de la face dors. sont général. petites el comrtes, mais, a la limite des faces dors. el ventr. it existe, de 
chaque cote du corps, une rangée de trés grosses papilles de dimensions uniformes et séparées régrul. par des intervalles égaux. Les boucles, rugueuses, sont de taille variable, mais elles n'atteignent pas les grandes dimensions qu'elles prennent parfois che\% l'H. tubulosa el lemrs orif. ne disparaissent jamais complet.

\section{*Il. stellati p. 17i}

31. Le corpos est divise en une rép. principale large el crlindrique, el une rég. term. beancoup plus étroite, formant me sorte de queue; il existe des org. arborescents. Forme tres ratre, vivant toujours a une certaine prof.

Molpadiu musculas p. KN

- Le corps est cylindrique, trés allongé el de forme régul, avec des tég. translucides; pas d'orgr. arborescents. Lisèces en général communes, ordin. littorales, vivant dans le sable ou le sable vaseux. F. Synaptidæ

32. Espèce extrêm. petite long. $10 \mathrm{~mm}$. portant 10 tentac simples: pas de sclér. dans les lég. trouvée une fois à St-Waast

Rhabdomolgus ruber p. 191

- Espèce pouvant alteindre de 10 a $25 \mathrm{~cm}$. : 11 ou 12 tentac. digités ou pinnés: les sclér. consistent en ancres articulées sur des

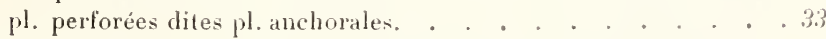

33. Tentac. pinnés, $c$ à $d$, offrant une partie principale qui porte sur ses bords un nombre variable de pinnules successives, au nombre de 6 à 9 paires arec, en plus, une pinnule terminale. Les pl. anchorales sont simplement ovalaires, rétrécies à l'extrém. sur laguelle s'articule l'ancre, el leurs gros orif. sont denticulés G. Leplosynapta

- Tentac. digités, c. à d. constitués par une lige principale portant à son extrém. seulement quelques ramifications 3 ou l en général). Les pl. anchorales ont la forme d'une raquetle, c. it d. sont munies à l'une des extrém. d'un manche sm lequel s'articule l'ancre; les orif. sont lisses G. Labidoplax.

3'. Pinnules lat. des tentac. au nomb. de 8 paires, subégales. Les ancres sont très développées el l'espèce athère fortement aux doigts; elle atteint une long. de $30 \mathrm{~cm}$. Pl. anchorales de moyeme dim., avec au moins 8 orif. princip. denticulés; les bords des pl. sont égal. dentic., au moins sur une partie de leur pourtour; la partie pointue sur laquelle s’insère l'ancre est séparée du reste par une saillie arrondie. . . . . . . Leptosynapta galiennei p. 1sti

- Pinnules lat. des tentac, an nomb, de fi pairesen général, plus une pinnule termin, plus gr. que les autres. Les pl. anchorales et les ancres sont médiocrement développées: l'esp. alhère pen aux doigts. Les pl, anchorales ont les bords lisses et possedent général. 7 orif. princip.; la partic pointue n'est pas siparée du reste par une saillie.

Leptosynapta mhirens p. 187 
35. Dans la partie ant. du corps, les pl. anchorales sont courtes, presque circulaires, avec un manche très court et des bords fortement denticulés: elles portent un réseau second.irrég. à mailles serrées el opaque, superposé au réseau principal. En s’éloignant de la rég. ant., le réseau second. disparaît. el les pl. s’allongent: elles deviennent ovalaires avec le manche assez allongé el des bords lisses mais un peu anguleux el elles offrent i a $b$ perfor. centr. plus rambles el d'aulres phus pelites: les ancres sont courtes . . . . . . . . Labidoplax thomsoni p. 1!)

- Les pl. anchorales sont toujours dépourvnes de réseau second.; elles sont seulement plus courtes dans la rég. ant. du corps que dans la rég. post. où elles se montrent toujours plus longues que larges; leurs bords sont toujours lisses; il existe ordin. 4 grandes perfor. centr. et quelques autres plus petites. Les ancres sont notablement plus longues que les pl. correspondantes.

Labidopla.x digitata p. 188

\section{S. CI. HOLOTHITIES PEDIFERES}

\section{O. DENDROCHIROTES.}

Holothuries pédifères chez lesquelles les leutac. sont ramifiés à la manièré des lranches d'un arbre; ces tentac. sont souvent an nombre de 10 mais parfois en nombre sup. à $1:$; le pharrux est muni de muscles rétracteurs spéciaux.

\section{G. CUCUMARIA Buinvili.e.}

10 tentac., dont 2 plus pelits silues du rote ventr. Les priclicelles sont disposés

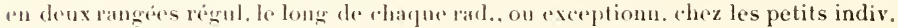
shivant une ligne en zigr-zag. I. interracl. sont orlin. dépourvus d'append., ou, s̈ils en possèdent, ccux-ci sont irrégulìr. dlistribués et général. plus petits que les pédicelles rad. L'anus est dépourvu de dents.

C. montagui Flemixg C. saxicola Briny et Robertsox]. Fig. 101. -

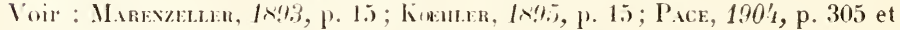
(1нтох, 191', p. 21 1, fig. 1, 1, f el 7 C'. saricola.

Le corps eylindrique alleint ordin, une long. de 10 il $12 \mathrm{~cm}$., il peut arriver 


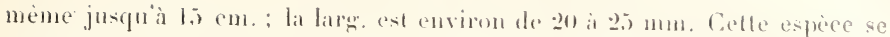

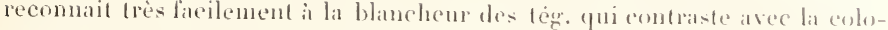

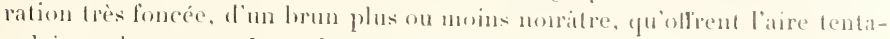
eulaire el les tentac. Les lubes ambul. sont disposes d'une maniere differente sur la face dors. el sur la face rentr. : sur les 3 rad. veutr. e esont des tubes

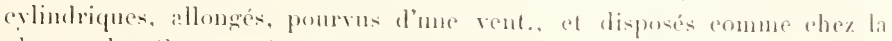

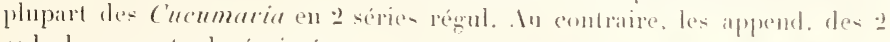
rad. dors. sont placés irrégulierr. arser cécartés les mon des antres. el ils forment des par pilles épaisses el coniques, munies cependant d'me petile vent. term. Iu point de vue anatomique, la C. montagui se distingue par la forme des tubes génit. qui sonl larmes el prea nombreus, avee l'extrém. élargie, en forme de poire comprimée. Les sclér. de la couche prof. des tég. ont d'abord la forme d'un biscuit plus ou moins ćtranglé en som milieu et offrant un orif. rers chaque extrém. élarque, ce qui les a fail désigner sous le nom de corpusc. en lunetle a ; les bords présentent par-

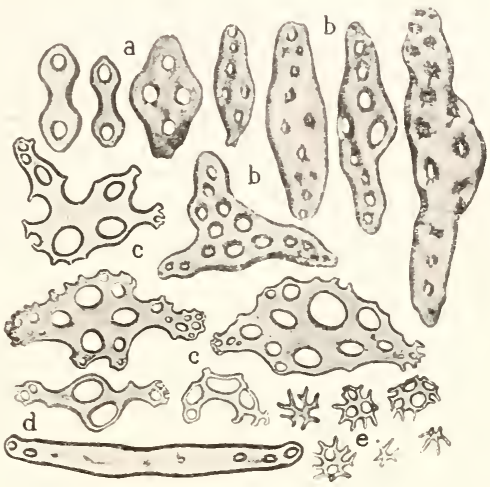

Fig. 101. - Cucumaria montagui: a, playnes en forme de lunette ou de losange, $X 120$; b. grandes plaques épassies; c, plarpues à réseau mince; $d$. bàtonnets des tentacules, $\times$ 70; e, corbeilles, $\times 120$. fois quelques petites pointes qui seront le point de départ de prolongements, et lorsque cens-ei en se développant arriveront is se rejoindre, les corpuse. prendront une forme losangique rég. avec í orif., mais sans nolules: cetle disposition régul. est conservée sur la plupart des corpuse.. mais quelques-uns cependant continuent à s'accroitre et domment naissance à des pl. irrégnl. el de dimensions variables, qui peurent meme atteindre 0.6 a $11.7 \mathrm{~mm}$. de Inng, mais restent toujours dépourvues de modules b . Jescorpust. superf sont des pelites corbeilles ineompletes donl le diam. varie entre 0.013 el $0.01 \mathrm{~mm}$. formées par quelques trabécules partant d'un mème fuint centr. recourbées el ordin. hifurquées mais non réuniés par un ecrele périph. e. Les append. ambul. el lestentac. renferment des bitomnets d el des pl. perforées à contoms irrégul. el conslifués par un résean calcaire d'épaisseur variable $\mathrm{c}$.

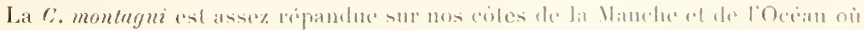

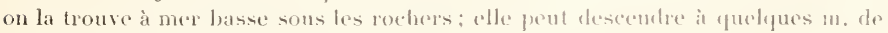
prof. Elle a été égal. observere en ditlópentes localités des Iles Britanniques, mais 
elle ne remonte pas beancoup vers le $\mathrm{X}$. On l'a reneontréaux Acores par $130 \mathrm{~m}$. de prof.

C. lefevrei Barrols 'C. normani Allex et l'ace. Fig. 102. - Voir: B.sroms / 1!1 1́, 1. 211. lig. 2, 5, 7 el s' C'. normani.

lat long. des échanl. varie ordin. entre fiel fo com. el peut même alteindre

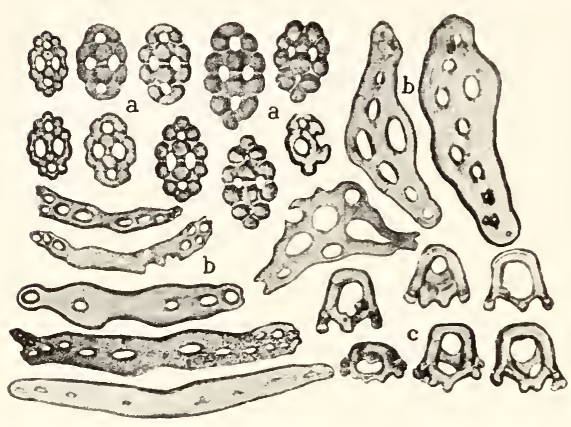

Fig. 102. - Cucumaria leferrei; a, platues à nodules; $\mathbf{b}$. batomets de diverses formes, $\times 130$; c, eorbeilles, $\times 190$.
$15 \mathrm{~cm}$. Le corps est cylindrique el la peau est epaisse, plus ou moins plissée, rugueuse et coriace. Les tubes ambul., rétracliles, forment 2 rangées distinctes dans chaque rad. et ils ont la même forme dans les j rad.; les interrad. ollrent des pédic. plus pelits et distribués très irrégulièr. Les tubes génit. sont très nombreux el peurent atleindre le chillre de .000 ; ils restent fins et evlindriques sur toute lem long.

less sclèr. des léğ. consistent principal. en pl. le plus souvent losangiques avec $\mathbf{1}$ grandes perfor. symétriquement disposées, portant, sur leurs 2 faces, de gros nodules arroudis el proéminents a les corbeilles, hémisphériques, sont constituées par des travées plutit fortes et épaisses el leurs dimensions sont relativ assez considérables car elles atteignent $0,07 \mathrm{~mm}$. de diam c Leur structure est beaucoup plus robuste que chez les espèces voisines avec lesquelles on peut confondre la $C$. leferrei, c. ì d. les C. montagui et planci. Les pédicelles renlerment des pl. identiques ì celles des tég., accompagnées de bâtomets de forme varialıle, allongés ou ramassés b: des bâtomnets analogues se trouvent dans les tentacules.

Les lég. sonl d'un brun assez clair chez l'animal qu'on vient de capturer: la couleur devient un peu plus foncéc a la lumiere: les tentac. el l'arre tentacul. sont brun foncé ou noirs.

Lat C. lefever se dislingue de la $C$. montagui par les append. ambul. formes de

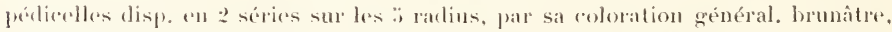

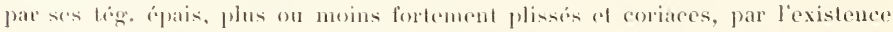

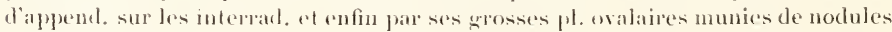
spheriques aree des perfor. spmétriguement disposies, Elle se distingue de la 
C. planci par ses pl. it nochules munies de i oril. symótriques, par ses corlecilles grandes et forles, ses lég. plissés ot la présenec diappend. dius les inferrat.

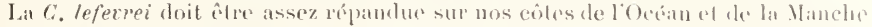

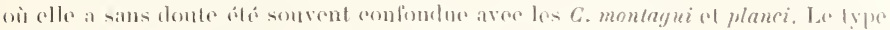

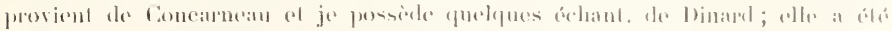

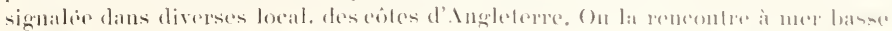

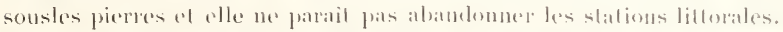

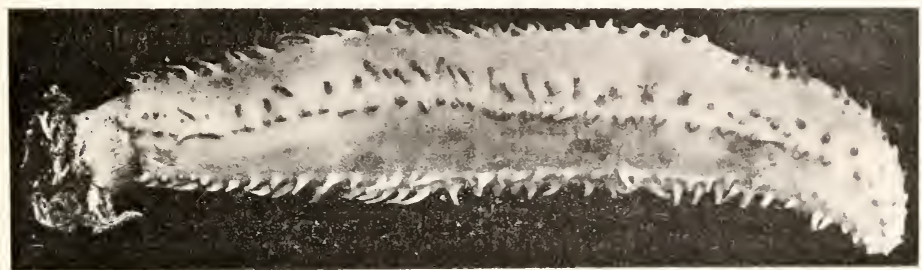

Fis. 103. - Cucumariu planci: animal entier: grandenr naturelle.

C. planci Brixnt . Fig. 103 el 104. - Voir : S.ın, $1 \times 57$, p. 120, pl. 1, lig.

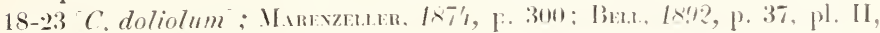
fig. 2- et pl. YIII, fig. 1.

Le corps est eylindrique ou quelque pen prismislique; il peut alleindre une loug. de 15 em. sur une lary. de 3 i 3,5 cm. Ia peau est assez épaisse el coriace, mais lisse. Les pédicelles forment 2- ramgeres bien distiucles dius les 5 rad., el les rég. inlerrad. sont complat. dépourves d’append. les lubes śénil.. assez. nombreux, sont lins. allongersel its resemblent a ceux de lal ('. leferrei.

l.es sclér. sont conslilués smotoul par des pl. "walaires, munies - 110 lenrs :faces de gros modules arromdis, avec des perlor: an

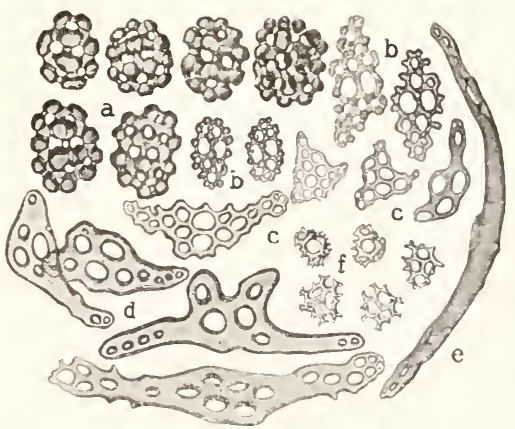

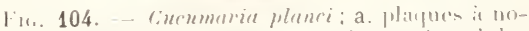
dules et is resean ipras ; b. plitgues a molules

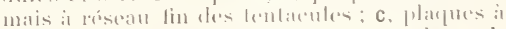

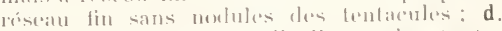

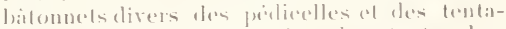

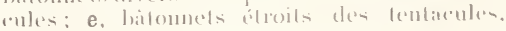
$\times 130 ; \mathrm{f}$, (o) recillus, X $190 \%$. nombre de 6 ou and desills

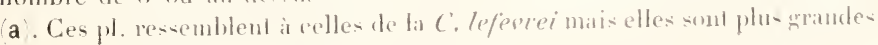


et les nodules ainsi que les perfor. sont moins symétriques el plus nombreux. Les corbeilles sont petites et ne mesurent pas plus de $0,01 \mathrm{~mm}$. de diam.; elles portent sur le bord de leur cercle term. un certain nombre de petites digitations parfois bifurquées $\mathbf{f}$. Les pédicelles renferment des pl. identiques à celles des tég., très nombreuses el serrés avee quelques bâtonnets munis de perforations $\mathbf{d}$. Les tentac. offrent les mèmes bâtonnet: ainsi que de très nombreuses pl., les unes munies de nodosilés arrondies mais à résean calcaire assez délical $b$, les autres dépourvues de nodules of lormées d'un résean assez fin, de forme triangulaire ou irrégul. c'.

La couleur générale du corps est d’un hrun plus ou moins clair les tentac. sont un peu plus foncés; tantiot la coloration reste uniforme, lantót il existe çà et là des taches plus claires.

La C. planci est extrêm. abondante dans toute la Méditerranée où elle vit général. dans les fonds vaseux ì partir de quelques m. do prof., mais elle peut descendre jusqu’à 60 ou $80 \mathrm{~m}$. : les pècheurs la capturent par grandes quantités et la nembrane péritonéale qu'ils arrachent après avoir ouvert l'animal teur sert d'appât pour leurs hameçons. Elle existe égal. dans l'Mtlantique et elle a été signalée depuis les côtes de Portugal jusqưà celles d'Angleterre, mais je suis persuadé quion l'a souvent confondue arec la $C$. lefevrei ou mème avec la $C$. montagui : il serait important de reviser les déterminations.

"C grubei Minexzeller C. dicquemarii SArs?. Fig. 105. - Voir : Sars. 1857, p. 125. pl. I, fig. 30-35: Marexzeller, 187', p. 305.

Le corps, de laille moyenne, alteint de 8 à $10 \mathrm{~cm}$. de long. sur 2,3 à 2,5

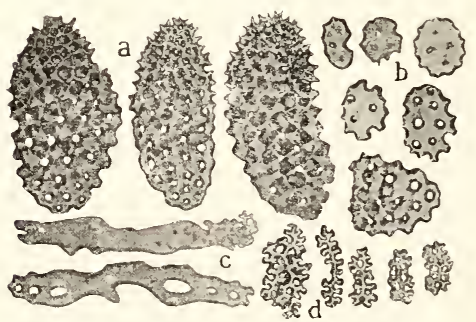

Fra. 105. - Cucumaria grubei; a, plaques en forme de cones de Sapin; b, états jeunes des plaques précédentes, $X$ i:i; c, batonnets, $\times 130 ; \mathrm{d}$, corpuseules crepus, $X 190$. de larg. ; il est eylindrique ou fusiforme et la peau est assez. mince. Les pédicelles, très rétractiles, restent localisés sur les radius: sur les 3 rad. ventr. ils sont disposés en 3 ou i rangrées, tandis que sur les 2 rad. dors., ils forment le plus souvent - rangées. Les sclér. consistent d'abord en zros corpusc. en forme de cones de sapin alteignant an moins 11. $1 \mathrm{mmm}$. de long. et munis de perfor. petiles très régul. disposées en quineonce; leur rég. term. amincie porte quelques epines coniques a. On trouve en ontre des pl. plus petites, a bords dentic., de laille et de forme variables, avec des orif. átroils, au nomb. de 3 ou i che\% les plus peelites dont la surf. est lisse b : les pl. plus grosices ont des oril. nomberex et presentent quelques nodules. Il existe, en plus, des bitcunets arqués ou nou qui se montrent surtout dans 
les pédicelles c : leur rég. centr. peut s'élargir el acquérir quelques perfor, Les tentac. renferment, en plus des bitmmets, des corpuse, crepus plus longs que larges $d$.

La couleur générale est jaune ou brun jannitre aree des laches blanches: lat face ventr. est plus claire.

La $C$.grubei, assez rare, na ancore été signalée que dans quelfues localités de

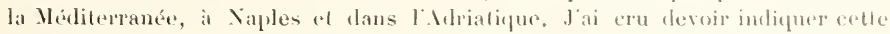

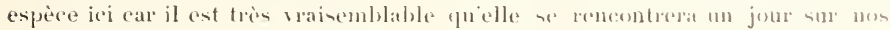

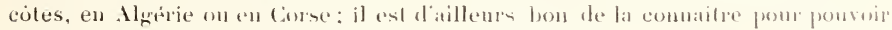
en distinguer la $C$. syracusana que jëhulie ci-rlessons.

*C. syracusana Grtbe. Fig. 106. - Voir : S.rns, 1857, P. 12:3, p]. I, fig. $24-29$.

Le corps e:t allongé, cylindrique, en forme de cornichon; il atteint 6 i 7 cm. de long. sur 1, i i 1,7 de larg. : lestég. sont assez corialcon mais lisses. Les nédicelles, lins et rétracliles. forment 2 rangées assez serrées sur chaque rad., mais il en existe anssi de plus fins épars dans les interrad. Les sclér. sont de $f$ sorles : cesont d'abord des pl. ou boucles épaisses, arrondies ou ovalaires munies de groses tubérosités et offraut de petits orif. b) ; des corpuse. plus gros en forme de cones de Sapin atteignant $0,5 \mathrm{~mm}$. de long. dont les perfor. sont petiles et irrégrulièr. alignées $\mathbf{a}$; des corpusc très petits se présentant souvent solls lorme de crois a 3 ou f branches c qui pewrent se rémir el former des pl. aplaties de forme variable $f$ el entin des corpusc. crépus. Les pélicelles renlerment des batomnets droits on arqués avec quelques pertor. el qui

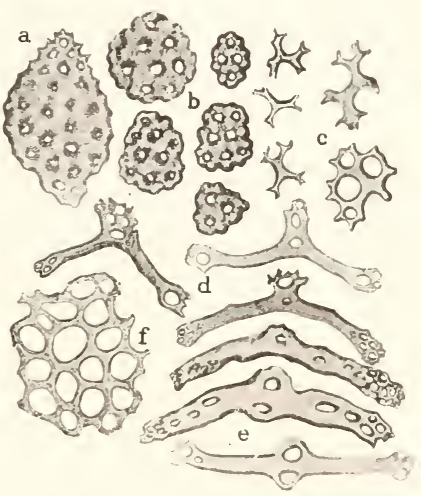

Fin. 106. - Cucumeria syraerusana; a, platgues en cones de sippin ; b. plitumes epaisses is conlour ovalatire, $X$ ii: c. formes jeunes de's plirgurs préceidentes, $X 190: \mathrm{d}$, bitommel avec lonrelle; e, bitomnet sans tourelle: f. platue it risean mince, Y. 130 . peuvent porter une fourelle rudi-

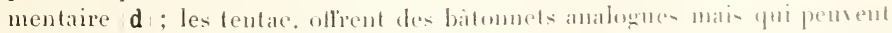

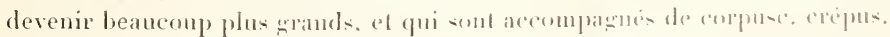

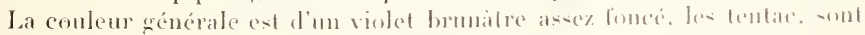

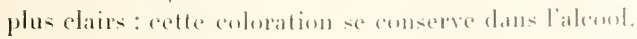

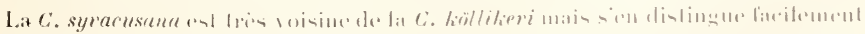

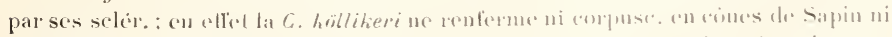

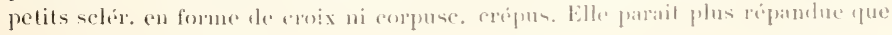


la $C$. grubei dont elle se distingue par les pédicelles formant 2 rangées sur les rad. dors. et par ses boucles arrondies munies de nodules.

La $C$. syracusana est surtout conme sur les cotes d'Italie et de sicile où elle a été rencontrée à diverses prof. jusqu ì $100 \mathrm{~m}$. Elle n’a jas encore été signalée sur nos côtes de lirance, mais jen ai reçu quelques exempl. de Tunisie (Sfax; elle doit se trou er égal. sur nos cótes d'Algérie el clle sera peut-ètre rencontrée un jour sur eelles de Corse.

*C. kollikeri SFarle, Fig. 107. - Voir : Sevipe, 1868, p. 237, pl. XXXIX. fig. 17.

Le corps ne dépasse pas 20 à $25 \mathrm{~mm}$. de long. sur 10 à $15 \mathrm{~mm}$. de larg. et

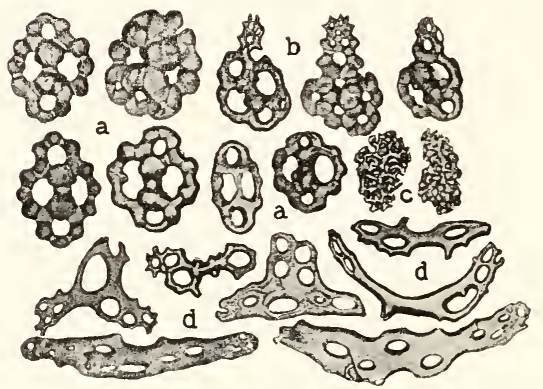

FIg. 107. - Cucumaria höllikeri; a, plapues épaisses des téguments à contour ovalaile; b, plaques avee un prolongement terminal; $\mathbf{c}$, corpuscules crépus: d, bấlonnets divers, \ 1:30. il est plus ou moins fortement recourbé en $\mathrm{U}$; les pédicelles sont régulièr. disposés en 2 rangées sur chaque rad. et il existe en outre un certain nombre d'append. interrad. plus petits el irrégulièr. distribués. Less sclér. consistent principal. en grandes $\mathrm{pl}$. ovalaires, munies de gros nodules sphériques assez régulièr. disposés et offrant des perfor. de dimensions variables a . Ces pl. présentent souvent une svmétrie régul. avec 4 orif.; mais chez plusieurs d'entre

elles, la rég. tournée vers l'ext. se prolonge en un processus plus ou moins marqué, sourent muni de quelques pointes b. disposition qui rappelle ce qui existe chez la C. lirischbergi. I.es pédicelles renferment, en plus de ces mènes pl., des batonnets tantit allongés, tantit triangulaires et passant a de véritables pl. irrérrul. d t. Les tentac. renferment des bâtonnets identiques à ceux des pédicelles el, en plus, des corpusc. crépus analogues à ceux de la C'. grubei, mais un peu plus délicats, plus petis et moins nombreux $\mathbf{c}$.

La conleur de l'animal vivant est d'un brun assez foncé el la face ventr. est jinuntre.

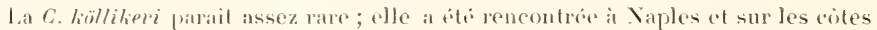
de Sieile: jen posside quelques exempl. provenaut du Portugal : elle peut done pansier dans listlantique, et il ast probable guon la reneontrera un jour sur nos

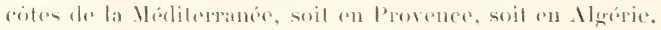

C. kirschbergi Hlater. Fig. 108. - Voir: Heular, 180r, p. 75, pl. 111, tig. 8 - 10 . 
Le corps est crlindrique, assey étroit. un pen aminci vers les extrémites: il ne me semble pas pouvoir alteindre de grandes dinem-ions et sa long. ne doit pas dépasser 30 mu. I.es tég. sont asiez résistants el ruguenx; les pédicelles, rétractiles, sont disposés régulier. sur 2a rangs dans chaque rad. Les tég. renferment des pl. assez grosses, pouvant attciudre 0, ímm, de Imug. sur 0,1 à 0,15 de largo. de forme irregulier. ovalaire et allongés ces pl. sont munies de perfor. formant ordin. 2 ramges principales, el il arrive fré souvent que l'une des extrém. se contimue en un prolongement étroit dans lequel les orif. sont plus petits ou disparaissent, et qui offre à sa périph. des pointes aiguës; sur d'autres pl., le prolongement. formé d'un tissu hvalin, siamincil progressivement en pointe et sa surf. est lisice. Les pédicelles renferment des bâtonnets de formes diverses (c et d et leur vent. term. offre une rosette calcaire bien développée. Marexzeluar a signalé en outre, dans les léx... de petits corpusc. crépus que je n'ai pas pu retrouver. Les tentac. renferment égal. des

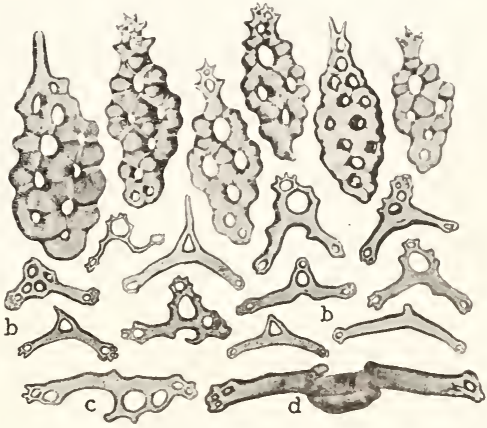

Fu. 108. - Cucumaria kirschbergi; a, plapues i nodules: b. britomets diver's aree tonrelle: c. bitomnet sans tourelle: $d$, liatonnet droit, $X 7 i$.

bâtounets recourbés dont la

partie médiane oflre souvent 1 ou 2 orif. et peut émettre 2 colonnettes convergentes qui portent, au point de leur réunion, 2 ou 3 petites pointes divergentes susceptibles de se réunir (b).

La couleur de l'animal vivant est d'un brun grisatre d'apré- Helter el d'un rouge cru d’après Hínocano.

La $C$. kirschbergi na encore éte trouvée, jusquä maintenant, qu en Méditerranée, elle ne parait pras très répandue: cependant IÉnot and dit quelle est assezz commune à Banyuls. Elle est roisine de la C. hyndmani que jeetudie ci-desius.

C. hyndmani Tuompsox . Fig. 109. - Voir : Bri., 189.2, p. 36. pl. II, fig. 1.

Le corps ne depasse général. pas fo mun, de longr.: il est asce\% large. cylindrique et peu aminci aux extrém. Les tég. sont durs et résistants. Les pédicelles sont assez régulièr. disposés en Z rangrées sur chaque rad., au moins dans les grands échant. Les sclér. consistent principal, en pl. grandes et épaisses atteignant $(1, \mathbf{f}$ à $(1,5 \mathrm{~mm}$, de long., de forme variable mais général. peu allongée, ovalaires on circulaires; ces pl, offrent de grosses perfor. disposées plus on moins régulièr. en séries longit. a . Il exi-te en outre de- 
batonnets recourbés du milieu desquels śélivent 22 colomnettes convergentes

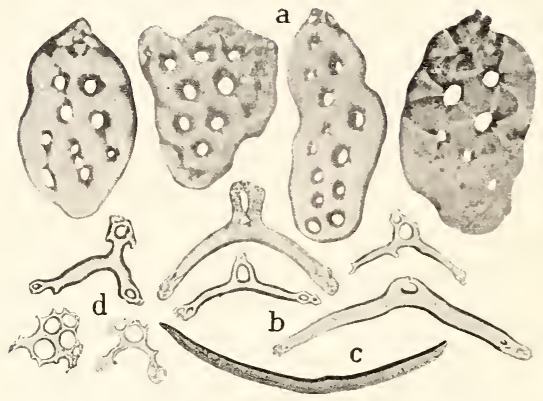

Fir. 109. - Cucumaria hyndmanni: a, plargues épaissies; b. bitonnets arec tomelle; c. bitonnet simple; $d$. plapues i réseau fin des tentacules, $\times$;i. portant 2 ou 3 petites pointes à leur point de remion. comme cela arrive che\% la $C$. lilischbergi b. Cies bitonnets se retronvent dans les pédicelles. Jestentac. renferment des bàtomnels allongés c), fantot lisses tantot munis de petites pointes: il existe en phus des batomuets offrant en teur milien 2 tiges convergentes el entin de petites pl. délicates à réseau irrégul. et très fin $(d$.

A létat rivant, l'animal offre une coloration jaunatre.

La $C$. hyndmani a été signalée en Méditerranépe ot principal. daus l'Adriatique où elle it toujours il une certaine prof., 010 m. at plus; elle a ité retrouvée a Banyuls daus la vase cótiere. Elle existe égal. sur uos cotes de la Vanche, où elle vit sous les pierres et les rocher's qui décourrent aux grandes marées; elle est assez commune sur les coles d'Angleterre et remonte mème jusqu'en Norvège.

Les C. hyndmani et hirschbergi sont assez voisines of on peut se demander s'il n'y aurait pas lieu de les réunir. Cependant le corps est plus mince, el plus allongé, et les pédicelles sont plus gros, plus courts et moins serrés chez la C. kirschbergi dont la coloration générale est rouge (l'après Iİnor arr); tandis que la $C$. hyndmani a le corps plus trapu, les pédicelles plus minces et une coloration jaunatre. Les pl. des tég. de la $C$. hyndmani sont plus grandes et plus larges, en outre elles nomt pas cette extrém. plus ou noins allongee et parfois munie de petits piq. qui termine souvent le's pl. chez la C. Firschbergi. On ne peut pas dire que la $C$. kirschbergi représente me forme mediterranemue de la $C$. hyndmani, car cette dernière, qui a surtout été rencontrée dans l'Allantique, a été également trouve dans lidelriatique ef à Banyuls. Toutefois, il y aurait lieu de rechercher sur des exempl. plus nombreus que ceux qui ont étr étudiés jusquà èe jour, sil nexisterait pas des formen de passage.

C. tergestina S.ık. Fig. 110 et 111. - Voir: SAHs, 1857, p. 127, pl. 1. lig. 36-38, pt. II, fig. 39 et io: R. Pl:rrier, 1902, p. 197, pl. XII, lig. s, pl. XXI, fig. 10-19 C. incurrata].

Le corps est plus on moins fortement incurve et il prend la forme soit d'un croissant soit d'un L'. avec la rég. movenne élargie et les deux extrém. 


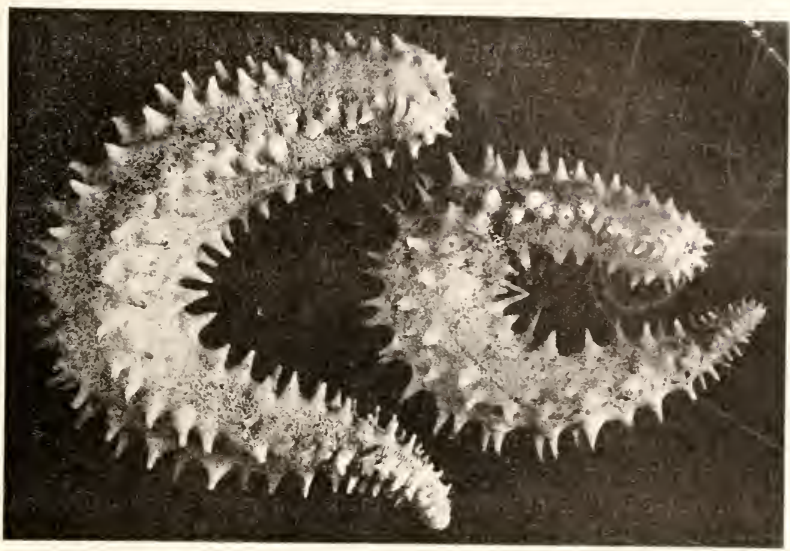

Fis. 110, - Curumaria tergestina, rue latérale de deux esemplaires différemment incurvés, X?.

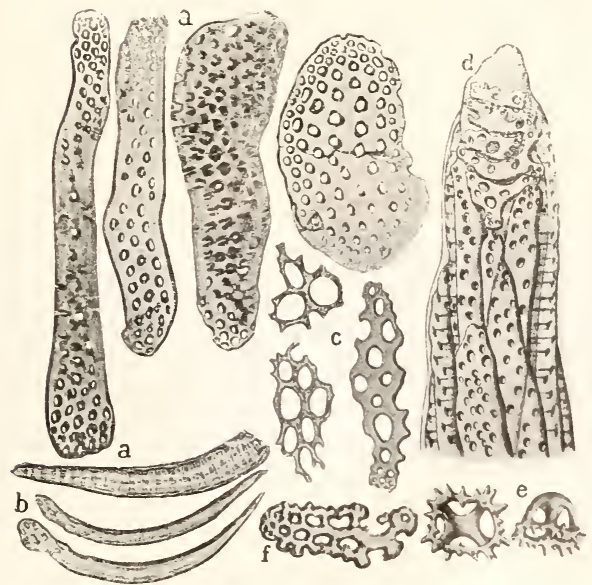

Fir. 111. Ciucumaria lergestina; a, plaques des téguments, l'inférieure vue de profil sur la moitié de sa longueur, $X$ it); b. bâtonucts: c. plaques minces, X io: d, extrémite d'un tube ambulacraire: e. colfeilles; f, corpuso

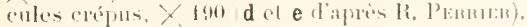




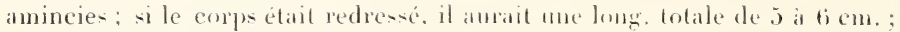
les pédicelles, asser gros, sont coniques, en liome de piq., dresses pointus, tout à fait rigides, fantot disposés sur? rangs. tantit formant une rangée en aig-rag sur ehaque rad.

Les sclèr, destén. consistent surtout en pl. perforess tris éparses el de grandes dimensions, de forme parfois irregul., mais général. très allongées, asse\% élroites et imbriquées a . Ces pl. atteignent l et mème 1,j mm. de long. ; leurs perlor. nombreuses, sont disposées en rangées obl. régul. el les intervalles qui separent ces rangees sont souvent assez sallanls, ee qui fait que les pl. paraisient cammelées. Les corbeilles sont de la forme ordin. et leur cercle porte de nombreux lohes courts el quelque peu renflés à l'extrém. e. Les pl. des tég. se retronvent daus les pédicelles mais elles y sont encore plus longues et plus étroites $\mathbf{d}$ ), et il existe en outre de petites pl. très comrtes, en forme de fer de flèche dont la pointe est dirigée vers le sommet dı pédicelle. La partie term. de celui-ci est dépourvue de sclér. et ne renferme même pas de disque: elle reste molle et llexible. Les tentac. oflrent surtout des bat. arqués, de dimensions et de formes diverses b). ainsi que de tres petites pl. de forme irrégul., armondies, triangulaires ou allongées, aree de grosses perfor. el un réseau delicat (c) : parfois les perfor. deviennent plus petites, et les bords des pl. oflrent des lobes arroudis qui leur domnent me certaine ressemblance avee des corpusc crépus f .

La couleur des indir virants est d'un jame brunctre pluson moins fonce, en partie conserve dans l"aleonl.

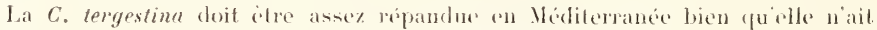
encore été rencontré qu'en exemple peu nombreux ; on la trouvere an large de Marseille, daus le sable vaneux ou dans les graviers coralligenes ver's :0 $\mathrm{m}$, de

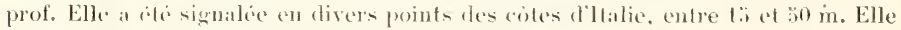

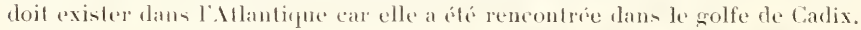

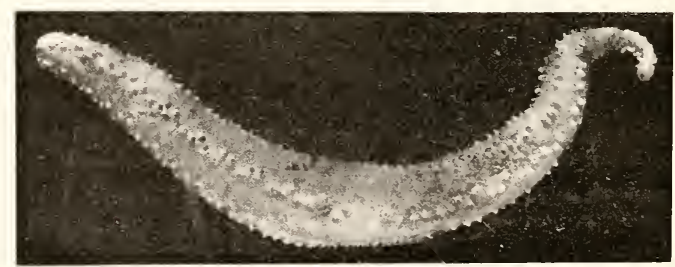

1.ig. 112. - Cucumaria elongata; rue latérale, $X 2,30$.

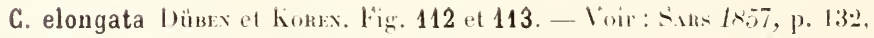

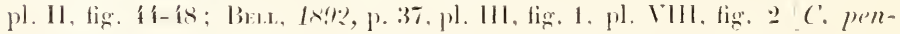

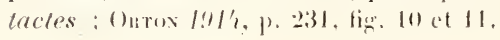


Le corps est allongé, cylindrique ou le plus souvent pentagonal el relativ. étroit, il peut atteindre jusqu’à 1.7 cm. de lonz.., mais la plupart des individus ont de 6 à $10 \mathrm{~cm}$, : la laron. varie entre $\{$ et 7 mun. Lal rég. ant. est légèr. amincie el la rég. post. se prolonge en se rétrécissint fortentent de manière à former me sorte de queue étroite et pointue. La peau est épaisise ef coriace en raison despl. calcaires très développées dont elle est bourrée. Les pédicelles, qui ne sont pas complèt. rétractiles, sont disposés sur z rangées dans chaque rad., du moins dans la rég. la plus large du corps, mais clans les parties ant. et post. plus étroites, ils sont placés en zig-zag. Les sclér, des tég. comprennent d'abord des pl, extrèm. grandes, de forme irrégul., souvent 2 fois plus longues que larges et qui penvenl atteindre 0,6 à $0,7 \mathrm{~mm}$. de long.; ces pl. sont assez épaisses el munies d'oril'. arrondis souvent disposés en rangées régul. (a ; d'autres pl, sont beaucoup' plus petites b. Les corbeilles des rég'. superf. des tég. sont lormées par í bittonnets principaux assez épais recourbés. réunis par un cercle portant toujours sur son bord libre plu-

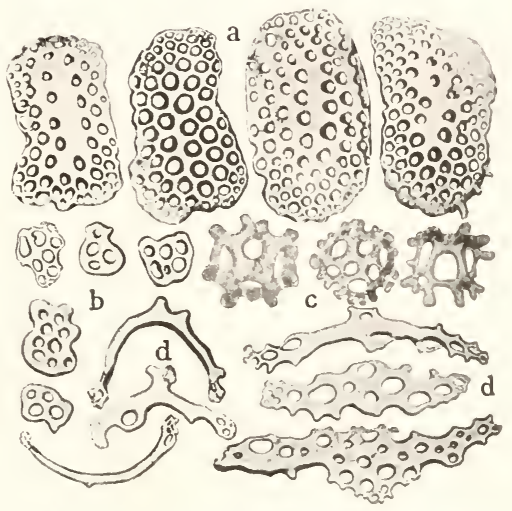

Fisi, 113. - Cincumaria elongata; a, grandes plaquesdestéguments, $X$ i 10 ; b. pelitesplaques, $X 130$ : c, corbeilles, $X 190 ; \mathrm{d}$, batonnets diver's, $\times 130$. sieurs piq. épais, allongés, à extrém. arrondie (c); il existe, en plus, des batonmels de diverses formes d ; ces mèmes sclér. se retrouvent dans les pédieclles. Les tentac. renferment surtout des bâtonnels arqués. munis de pointes el fonruissant souvent des ramilications.

A l'état vivant, la $C$. elongata présente une coloration brunàtre ou grrisatre plus au moins foncée, qui disparait dans l'alcool.

La C. elongata se reneontre surtont dans le sable où elle vit it moitié enfoncée par sa rég. ant.; dans l'Atlantique on la rencontre aux grandes mares dans le

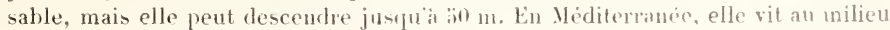
des Algues, parfois à quelques 112 . de prof., mais elle peut ausi alteindre in ou $50 \mathrm{~m}$.

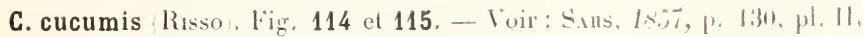
fig. $\$ 1-\{3$.

La long, varie entre 5 et $6 \mathrm{~cm}$. sur 10 à $15 \mathrm{~mm}$. de larg, : le corps est 
souvent recourbé sur lui-même en forme d' $\mathrm{L}^{\circ}$; la partie médiane est assez élargie el les 2 extrém. vont en se rétrécissant progressivement. Les pédicelles coniques et printus, assez petils et serrés, ne sont pas rétractiles el olfrent une petite vent. term.; ils forment sur chaque radius 2 rangées distinetes, mais vers les extrém. ils sont disposes en zig-zang : il n'y a pas la moindre indication d'append. dans les interrad. Les leg. renferment de grosses pl. assez. éparsses, de forme irrégul. el ressemblant à celles de la $C$. elongata tout en restant toujours plus petites el moins regul. que chez cetle derniere, avec des perfor. centr. tris srosses el ponvant former 3 ou í rangées parallèles (a). 1)antres pl. sont beancoup phus petites et sont identiques aux petites pl. de la C: elongata. Les corbeilles, petites, sont constiluées par des trabécules lormant un réseau irrégulier, réunies par un cercle lisse ou ollrant de petits lobes peu nombreux, courts el arondis, mats jamais de dents allongeses comme la $C$. elongala c. Les pédicelles renlement les mèmes corpuse. que les tég., avee, en plus, de petits batomels quion retrouve égal. dans les tentac. (b).

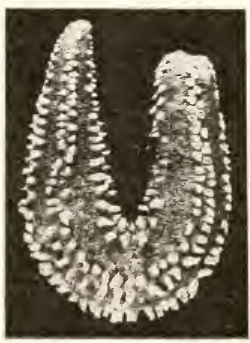

Fis. 114. - Curumaria curnmis; vue latérale, $X 2$.

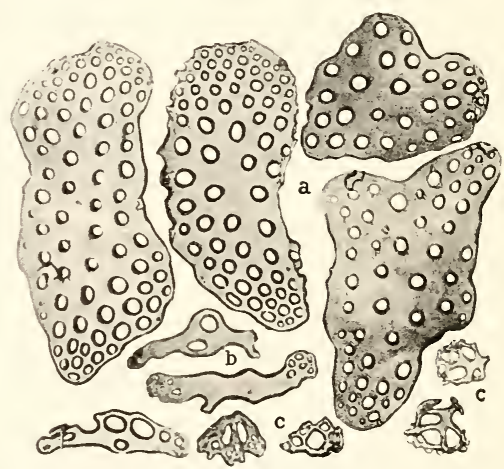

Fiti, 115. - Cucumaria cucumis; a, grandes plaques des téguments, $X 7 \ddot{3} ; \mathbf{b}$, bàtonnets, $\times 190 ;$ c, corbeilles, $\times 190$.

Lat conkul chez l'animal vivant est assez foncée, brun noiratre ou olivitre, ave la lice ventrale plus chare; elle se conserve en partie dans l'aleool.

La C. cucumis est trés roisine de la G. elongata; elle en dittire tontefois par son corps beancoup plus raceourci ef nollrant jamais te prolongement en forme de queue qui caractérise celle-ci. Les pl. des legg. sont plus petites el moins allongées que chez la C. elongatu; enfin le cercle des corbeilles est a peu près lisse.

lat $C$. curumis a surtoul ele rencontrée dans diverses localiles de Idriatique

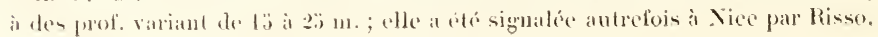




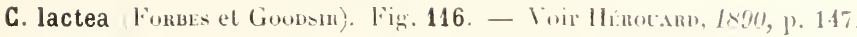
pl. XXXI, fig. E.

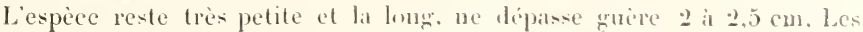
lubes ambul. sont disposis en rigr-zalg sur chaque rad.; leur nombre varie d'ailleurs avee les échant, mais, d'une manière grénérale, les append, des 2 rad. dors. sont moins nombreux que ceux des 3 rangées ventr. Les tég. sont asse\% rigides en raison du nombre et de la taille des enrpuse, calc. qu'ils renferment. Ces tég. sont blancs, tantìt d'un blanc très pur, tantot légèr. rosés; les tentac. sont jaunes. Les selér. consistent d'abord en pl. ovalaires rappelant, par leurs nodules arrondis, eelles de la C', planci (a). Lat plupart offrent 2 perfor. centr. assez grandes el 2 autres plus petites disposées en croix par rapport aux précédentes; d'autres pl. sont plus graudes, irrégul. et leurs perfor., plus nombreuses, sont grandes et inégales (b) ; certaines d'entre elles se développent eneore davantage, mais elles perdent eomplèt. leurs nodules: elles restent dès lors aplaties avec un contuur irrégul., des mailles assez épaisses et de gros orifices; leur long. peut alteindre $1,5 \mathrm{~mm}$. Il existe égal. des pl. à réseau plus délicat el de forme irrégul. c : lorsque ces pl. deviennent plus longues et plus étroites, elles prement la forme de batonnets (d). Enfin la couche superf. des lég. renferme des pl. très petites, sortes de corbeilles aplaties ofrant quelques travées centrales disposées souvent en croix, avec de très fines expansions périphér.; celles-ci peuvent se ramitier mais ne se réunissent jamais en réseau e. Les pédicelles el les tentae. renferment des bâlonnels assaciés à de petites pl. constituées par un réseau calcaire délicat.

Fif. 116. - Cucumaria lactea; a, plaques avec orifices strmétriques et munies de nodules; b, plaques plus grandes el irrégulières, $\times 130$; c, petiles playues irrégulières passant it des batonnets : $d$. bitonnets, $\times 19 \theta$; e, corbeille (d'apres Hénocaulo), X280.

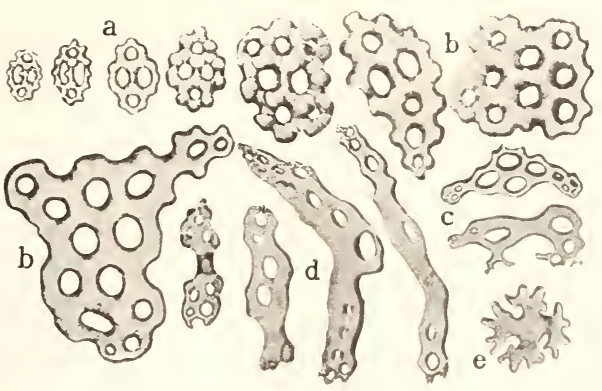

La $C$. lactea doit exister dans diverses focalites de nos coten de lat Janche et sans doute anssi dans l'Océan; mais jusqüi preisent elle n'a éti anthentiquement

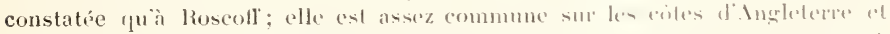

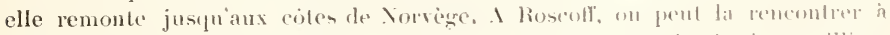

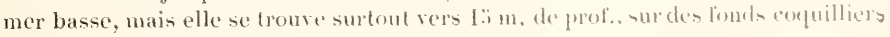
ou parmi les Algues ealcaires, 
G. brunnea Forbes. Fïg. 117. - Voir: Hérotard, 1890, p. 148, pl. XXXI, fig. B).

La C' brumnea présente la mème forme ext. que la C. lactea : mêmes dimensions l ré réduites et mème disposition des append. ambul. ; la couleur

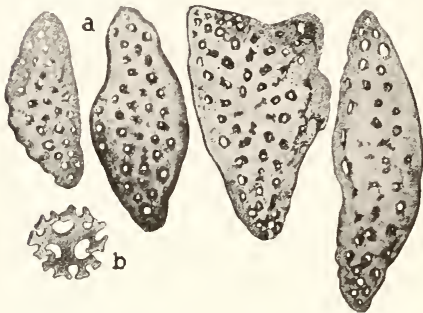

Fis, 117. Coucumaria brunnea: a, grandes plaques des téguments, X $73 ;$; b, corbeille (l'après Ilénot.116, $X 280$. seule dillere et varie, chez la première, du brun clair au brun foncé: celte difrérence dans la coloration permet de distinguer immédiatement les 2 esp. the plus, les sclér. ne sont fats identiques: ils consistent chez la C. Lrunnea en pl. ovalaires munies de tuberc. arrondis el présentant im nombre de perfor. général. assez élevé, nulliant que rarement la disposition régul. el srmétrique des petites pl. de la C. lavleu, mais rappelant les pl. plus grosises comme celles de la lig. 116, b. Il existe en outre des pl, grandes 11,5 à $0,6 \mathrm{~mm}$. de long.), épaisses, de forme variable ovalaire, fusiforme, triangulaire, etc. , a perlor. nombreuses el pelites. disposées en rangrées longit. régul. : ces pl. manquent complet. chez la C. lactea. Enlin le cercle périphér, des conbeilles donne naissance a de petits lobes souvent bilurqués el pouvant même se réunir et se souder aux lobes voisins (b).

La $C$. brunnea est général, associée sur nos cótes de la Manche à la $C$. lactea, à laquelle la réunissent Bell, 1892 , p. 38, MAssy, 1920 , p. 46, etc., et se troure comme clle sur les Algues calcaires vers $15 \mathrm{~m}$. de prof, ou mème à la còte aux grandes marées. Elle se rencontre aussi en Méditerranée où la C. lactea est inconnue, et elle nest pas rare i Banyuls. On la connait sur les cotes d'Angleterre, mais elle ne parait pas remonter aussi hant vers le $X$. que la G. lactea.

\section{(i. THYONE OKEX.}

Dendrochirote possédant 10 tentac., les 2 ventr. plus petits; les pédicelles sont nombreux et répartis uniforménent sur tout le corps sans qu'on puisse distinguer de rangées rad. régul. ; l’anus est souvent muni de dents.

T. fusus O. F. Mïller. Fig. 118. - Voir: Sans, 18:7, p. 135, pl. 1I,

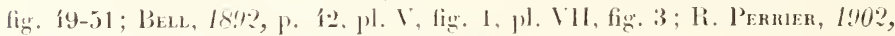
p. 510 , pl. XXI, lig. 29-31|Th. gadeana.

Le corps peul atleindre une grande taille el arriver à $20 \mathrm{~cm}$. de long. sur une larg. de 3 à $\mathrm{i} \mathrm{cm}$., mais habituellement la long. ne dépasse pas $10 \mathrm{~cm}$. la forme est cylindrique avec les deux cxtrém. amincies. Les pédicelles sont souvent groupés de manière à former des rangées longil. assez apparentes, ansi bien sur les rad, que sur les interrad.; l'anus est entoné de 
5 dents calcaires. Les -clér. comsistent en corpuse. lurviformes tres simples. dont le disque, qéneral. oralaire, partois allongé, oflre le plus sonvent 4 orif. plus grands el quelques autres plus petits: de la rég. centr. sélèvent ? colonuettes étroites et comvergentes qui penvent se terminer par '2 on 3 pointes a . Ces corpusc, se retrousent dans les pédicelles. Les tentae, renferment des bàtonnets étroils c et de petites pl. irrégul. constituées par un réseau calcaire délical (b).

la eouleur à l'état vivant est blanchàtre ou rosée.

La forme des corpuse. Iurriformes sépare complèl. la $T$. fusus des antres especes françaises du g. Thyone.

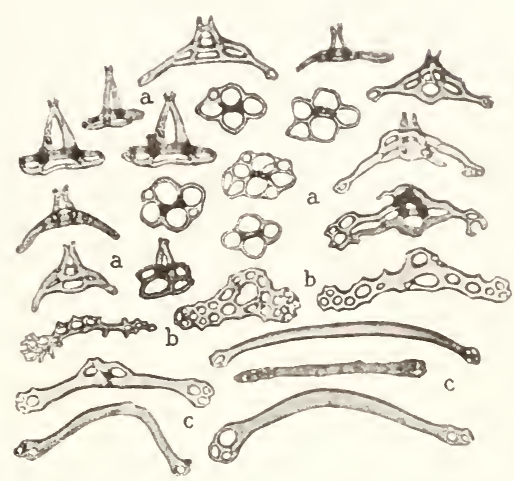

Fig. 118. Thyone fisus: a, corpuscules turriformes, $X 130$; b. plaques it réseau délicat: c. bittonnet, $\times 190$.

La $T$. fusus est très répandue en Véditerranée et dans l'Allantique. Elle a été trourée dans un asser grand

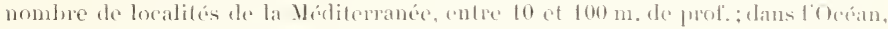

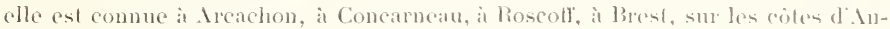
gleterre, etc, ; elle remonte au $\mathrm{Y}$. juscuinen Yorrige.

T. raphanus Dübex et Korex. Fig. 119 et 120. - Voir : Beur. Ixg?, p. 42,

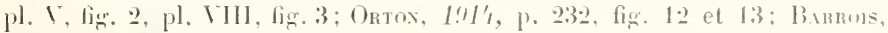
1.8\%, 1. 53 Th. poucheti.

Le corps est plus ou moins fortement incurve, il ślargit rapidement

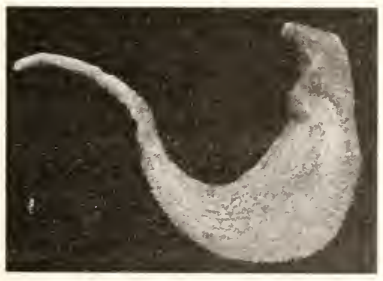

Fig. 119. - Thyone raphanus, vue latérale, $X:$.? a partir de la bouche pour atteindre sa larg. maxima quill conserve sur prí de la moitie de sa loniz.., puir il s'allénue progresivement en win long procestr. eandal qui reste tre nince. lichant. que je reprétente ici fig. 119) anrail, si on le rederesit emplet., nue long. totiale de fo com.. mais général. les is div. sont moins grands. Les pédicelles se troment repartis sor tomt le corpe moins nombreus sm lat rég. dors. ed sur le procestur candial. et ils disparaisent complet. an voisinage de l'anus qui est entouré par 5 grosses dents. Les lég. renferment surtont des pl. 
aplaties el lisses, à contour arrondi, et munies de grosses perfor qui

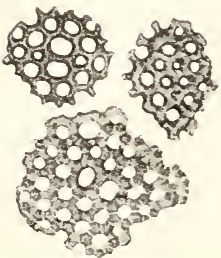

Fig. 120. - Thyone raphanus; placfues destéguments, $\times$ i:. alteignent presque $1 \mathrm{~mm}$, de diam. fig. 120. A ces grosses pl. sont associés de petits corpusc. crépus; au voisinage des dents anales, les pl. deviennent plus allongées el plus fortes et leur surf. est ordin. mamelonncée.

La coulenr est jaumatre ou brunatre assez claire (hez l'animal vivant: elle disparait dans l'alcool.

Lit $T$. raphanus est surtout connue lans les mers du X., sur les côtes de Norvìge, aux ìles Faroë et Shetland, et sur les cotes l'Angleterre, à des prof. variant de i a $900 \mathrm{~m}$. Elle a été retrouvée par Marios, au large de Marscille, ¿ los m. de prof. On la reneontrera (cortainement un jour sur nos cotes atlantiques. 1) ait-

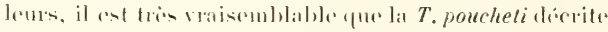

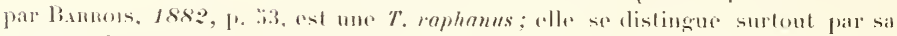

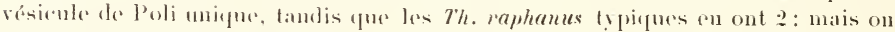

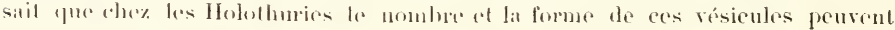
présenter des variations.

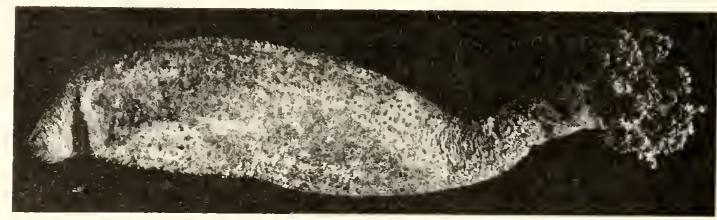

Fis. 121. - Thyone roscovila; vne latérale, légèrement réduit.

T. roscovita Héroctro. Fig. 121 el 122. - Voir: Hérolind 1790, p. 152. pl. XXXII, fig. 6, 15 el 16 ; Cix́ot, 1!1?, p. 59.

Le corps étalé mesure 7 à $8 \mathrm{~cm}$. de long. el les tég. sont d'un gris rosé piqueté de brun, non transparents. Les sclér. font complèt. défaut dans les tentac. ef les pédicelles: ils n'existent que dans les 111 tubes ambul. term. entourant l'anus où ils se monlrent sous forme de bâlonnets droits ou recourbés, avec

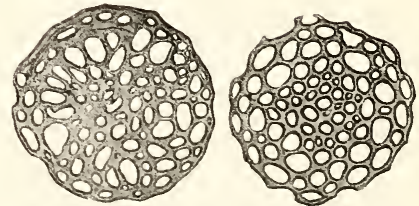

Fig. 122. - Thyone roscorita: disques calcaires des lules ambulacraires. quelques perfor. term. Les pédicelles renferment dans leur ventouse un disque ealcaire bien développé (fir. 122). l'anus présente 5 denls rad. trimgulaires ì côtés échancrés.

La $T$. roscovita n a eneore áte rencontré que sur nos côtes de lidlantique ou 


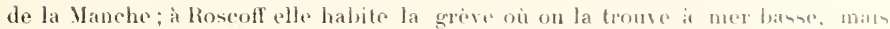
elle peut descendre jusque vers $40 \mathrm{~m}$; 0 on la comait au large d'Arearlon; je possède également un échant. trouvé à mer basse à Dinard.

La $T$. roscovita se distingue facilement de fat $T$. inermis par ses tég. opatques et d'une couleur grise ou bronatre et par l'alsence complite de corpuse. lurriformes an roisinage de l'anms.

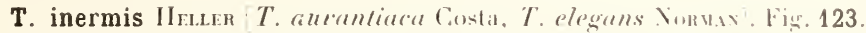

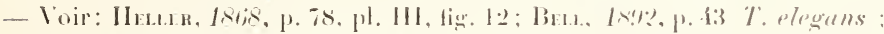

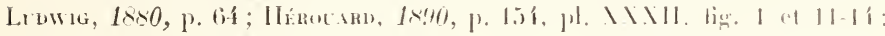
Cúxor, 191?, p. 59 T. aurantiaca.

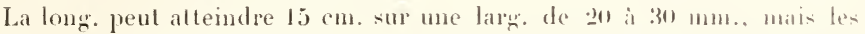

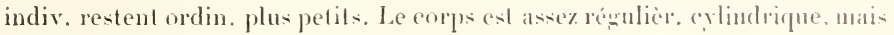
il devient fusiforme lorsqu'il est confracte. Les parois sont tout à fait transparentes et minees, tres délicates, de couleur rouge on rosée, et les pédicelles, irrégulièr. répartis sur toute la surf., sont assez serrés. Les sclér. n’existent que dans la rég. post. au voisinage de l'anus: ce sont des corpusc. turriformes, plus ou moins complèt. développés, dont le disque est tantôt circulaire, tantôl irrégulier (b); la plupart de ces disques portent 2 colomettes courtes, terminées par quelyues pointes et convergentes; d'autres disques portent un nombre variable de colonnettes isolées ou de saillies à forme variable:

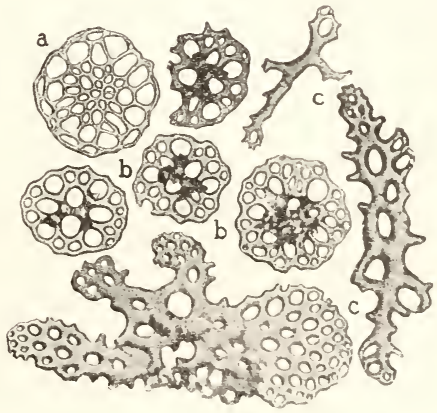

Fra. 123, - Thyone inermis; a, disfue calcaire des tubes ambulacraires; b, corpuseules turriformes: c, grosses plaques irrégulieres de l'extrémité posté rieure du corps, $\times$ t 30 . d'autres enfin sont rérluits exclu-

sivement au disque basilaire. Les pédicelles ne renlerment qu'un disque calc. terminal (a). Lanus présente 5 dents épaisses, mulraclueuses. hérissées de piq., aceompagnées de grandes pl. irrégul. el inéz̆. parloís éparimien par un réseau secondaire (c)

La $T$. inermis se distingue facilement de lit $T$. roscorita pitl la minceur de ses tég., roses chez l'animal vivant; clle na encore été signalée jusqü it précent qui en Méditerranée où elle parait dailleurs tris rare; on l'a rencontrón à Naples ot it Messine; Hérotaro l’indique à Banyuls, je l’a moi-míme dragure ì Cette, dans des fonds vaseux, à $30 \mathrm{~m}$. (he prof. 11 est très probalude que linspiece existe dans la Manche et que la Thyone décrite par Yorman sous le nom de T. elegans nést antre qu'une $T$. inermis. 


\section{G. PSEUDOCUCUMIS LUDWIG.}

18 tentac., 10 plus grands et 8 i 10 plus petits alternant avec les premiers ou formant un cercle int. Les pédicelles sont disposés en 2 rangées principales régul. le long des rad. et ils deviennent plus nombreux vers le milieu du corps ; les interrad. sont en principe nus, mais frarfois ils sont oceupés par quelques pédicelles vers le milien du eorps. Les pieces de l'anneau calcaire pharyngien sont tres

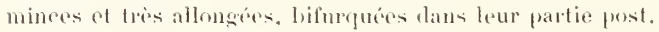

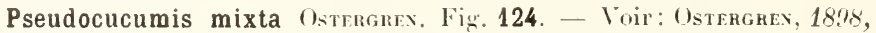
p. 135 et 1906, p. 1. fig. I et 3 ; Kommen et Vixfy, 1905, p. 395, fig. 1 à 6 [Ps. cuenoli].

Le corps est allongé, légèr. fusiforme: la long. totale peut alleindre 15 à $20 \mathrm{~cm}$. avee un diam. de 25 a $30 \mathrm{~mm}$. dans la rég. moyenne. Dans les petits échant. les pédicelles sont disposés sur உ rangées dans chaque rad., mais leur nombre angmente dans la rém. movenue du corps où ils forment de 2 à f rangrées; chez les grands indir, ils sont d'abord disposés sur 2 rangées, puis lemr

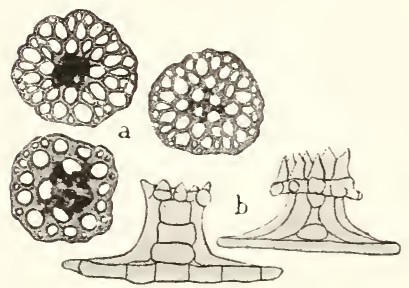

Fig. 124. - Pseudocucumis mixta; a, corpusenles turriformes vus de face, $\times 130 ; \mathrm{b}$, corpuscules turriformes de profil (d'après Konuen et Vixis), $\times 200$. travées transs. Les pédicelles renferment outre les corpuse. lurriformes, des pl. assez grandes, minces. it contonr irrég. el simnenx. Les tentac. possiedent des bitomnets anquels sont associées de petites pl. arrondies et dex corpusc. turril. souvent incomplests.

Lin conleur des exemplaires en alconl est blanchitre ou brun grisâtre, avec des laches brunes assez foncées lres irrégul. el plus ou moins élendues.

Le $P$. mixla est répandu sur les cottes oecitentales de l'Europe et peut même devenir très abondant daus certaines locatités: a l'ile de Tatihou, Ostergnex en a ru des quantités considérables rejotíes à la cite après une tempête. L'espèce a été également trourée à Areachon. à Bréhat et a Wimereux; je suis persuadé 
qu'elle sera souvent reneontréc sur nos cotes lol'squ'on saurat la reennaitre, mais elle a du ètre fréquemment eonfondue avee diuntres. Ifolothuries; ello a été signalée sur les coites de Norvìge ot des Farö̈.

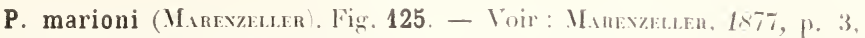
fig. 1. [Curumarin m.].

Dans l'exempl. type, le corps avail sententent fi mm. do lome. of 3 mum. de larg. vers te milien: l'espiece reste toujums de tròs petite taille. Les tég. sont durs al résistants; les pédicelles sont lisposés asse\% régulièr. sur 2 rangées avec cependant une certaine tendance à alterner; ils manquent sur les interrad. Dans sa description originale, Marexzelone indiquait que lestentac. étaient

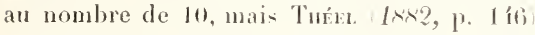
nous informe que Marenzeller a trouvé, après un nouvel examen, qüindépendamment des 10 grands tentac. mentionnés d'abord par

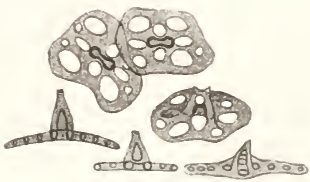

Fig, 125. - Pseudorucumis marioni; corpuseubes turriformes lapues M.mm:zr:II.5u, $X 220$. lui -.. disposition qui lni avait fait placer

l'espèce dans le g. Cucumariu - il existe lo autres tentac. plus petils, ce qui porte leur nombre a 20 en tout. Tuis. proposatit de placer l'espéce dans le g. Thyonidium : ce dernier g. a disparu et, il mon aris. l'espece doit rentrer dans le g. Pseudocucumis.

Les corpuse. turriformes des tég. ont un disque basilaire relativ. grand el irrégul. arrondi ou ovalaire, avec plusieurs perfor. assez prandes; les tourelles sont composées de deux colonneltes sentement, convergentes et munies à leur point de reneontre de quelques petites spinules. Inos les pédicelles, les disques hasilaires sont plus allongés.

L'espèce a été découverte par Mamox dans le golfe de Matreille oi che vit

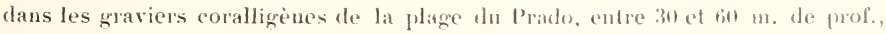

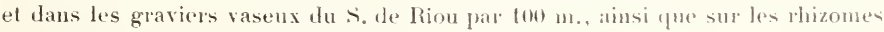
de-Posidonies au N. de Tiboulen, à 20 m, semlement.

\section{G. PHYLLOPHORUS (iش'Bl:.}

13 à 20 tentac. inégaux, les 5 it 10 plus petits alternant avec les 10 plus graurls ou formant un cerele int.; les pédicelles sont répartis uniformément sur tout le corps: pas de dents anales.

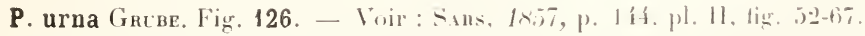

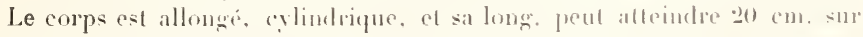

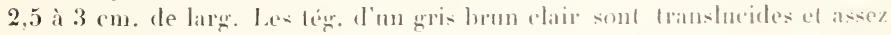
minces. I.es appendices sous forme de tubes, sout répartis sur tout le corps et 
assez serrés. I.es grands tentac. peuvent atteindre 3 i $3,5 \mathrm{~cm}$. de long., leurs ramifications sont nombreuses, allongées et asse\% minces.

Les sclér. des tég. consistent surtout en pl. perforées, de forme irrégul.,

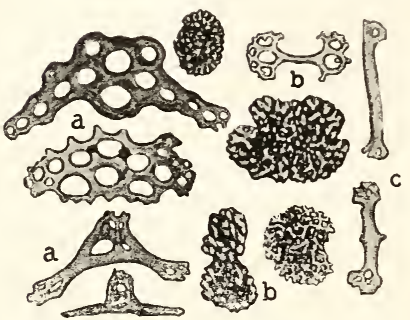

Fis. 126. - Phyllophorus urna; a, plaques avee ou sans tourelle rudimentaire, $X 130 ; \mathbf{b}$, corpuscules erépus; c, bitonnets, $\times 190$. général. plus longues que larges, atteignant 0,3 à 0,35 de long., ì perfor. inégales et assez grosses a. Parmi ces pl., on en rencontre quelques-mes de forme somvent arrondie el plus petites, de la rég. centr. desquelles s'élèvent $\mathbf{4}$ petites tiges vertic. réunies par 2 travées transv. constituant ainsi un petit corpusc. Lurriforme. Il existe en outre de très nombreux corpuse. crépus doml le diant. moven est de $0.116 \mathrm{~mm}$. b ; ces mèmes corpusc. se retrouvent dans les pédicelles. Les lentac. renferment de nom-

breux bàtomets c a ainsi que des corpusc. crépus.

Le P. urna na eneore étí trouví qu'en Misliterranée el prineipal. à Narseille;

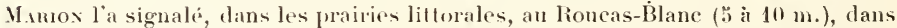
la calanque de Ratonneau ( $2-3$ m.) et dans les fonds vaseux au large de Niolon, ainsi que dans l'avant-port $\mathrm{X}$. du bassin de la Joliette. L'espèce a égal. été rencontrée à Banyuls ef à Naples, toujours ì dassez faibles prof.

\section{1). ASPIDOCHIROTES}

Holothuries pédifères chez lesquelles les ramifications des tentacules sont peltées et forment une sorte de disque termin.; pas de muscles rétracteurs spéciaux pour le pharynx.

\section{F. HOLOTHURIIDÆ LUDWIG.}

L.e corps est allongé, cylinlrique, avec une sole plantaire en général peu accusée: les canaux aquifères allant aux tentac. possèdent à leur base chacun une 


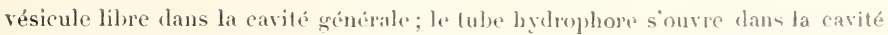
générale; l'organe arborescent gatlehe est entoure d'un réseatu. Formes le plus sowrent littorales.

\section{G. HOLOTHURIA Lix́.}

Aspidochirotes possedant une vinglaine de tentae. subigatux: les append.

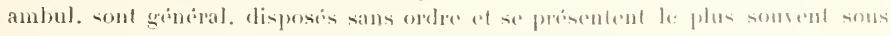

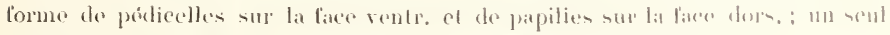
faiseeau de tubes génit. placé à gauche du ménentère dors.

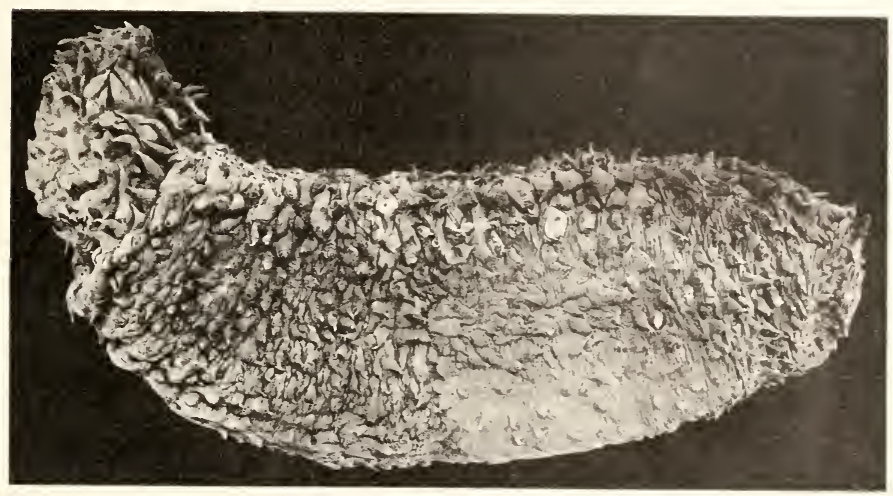

Fis. 127. - Holothuria sanctori; échantillon de Vaples en aleool, $X 12$.

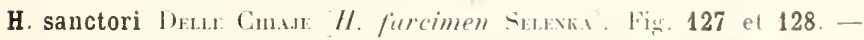
Voir: R. Perniler, 190?, p. 47\%, pl. XV, lig. 15-27 II. farcimen.

Le corps est presque eylindrique, assez aplati sur la face ventr.. il mestre 15 à 20 cm. de long. sur j cm. de larg. dans les exempl. conserves. mais il doit ètre sensiblement plus grand che\% l'animal vivant. La lace ventr. est couverte de pédicelles extrèm. serrés el formant un revetement uniforme dans lequel il est impossible de recomnaitre des rangés distinetes fig. 127. La face dors. est eowverte de tubere. trè- développés. coniques at allongé-. termin. par une papille mince et pointue. Entre les luberc.. se trourent des append. beancoup plus petits qui representent des pédicelles. Lorsque les tuberc. dors. sont fortement eontraetés lenr aspect. comme la lait remarquer R. Perrien, rappelle la trace que laissent les radicelles adventivessur la base rentlée d'une lige de biambon. La limite entre les pédieclles rentr. et les tuberc. dors. est très nette et les premiers tubere, margin. sont identiques 
à ceux du reste de la face dors. Il existe un org. de Cuvier formé par de tubes fins et allongés réunis en un long faisceau.

Les sclér. consistent d'abord en corpusc. turriformes à bords légèrement festonnés et lisses ; autour de la perfor. centr. existe un cercle de 8 à 12 orif. périphér. à peu près égaux, et en dehors de ce premier c'ercle il peut en exister un $2^{m e}$ constilué par des perfor. beaucoup plus petites fig. 128 a et 1 ). La tourelle, épaisse, avec 2 élages de travées transi., est plus courte que le diam. du disque. On troure en ontre des boucles planes, de forme ordin. ovalaire, pereées de perfor, régulier. disposées en 2 rangées, el au nombre de 3 ¿ \& dans chaque rangée b. Les papille-dors, offrent au--i des corpusc.

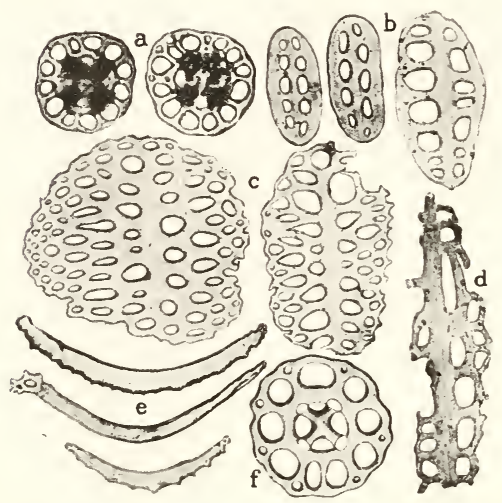

Fig. 128. - Holothuria Sanctori; a, eorpuscules turriformes. $\times 190 ; \mathrm{b}$, boncles petites formes) ; c, boucles (grandes formes), $\times 130 ; \mathrm{d}$, bitommet alargi et perforé, $\times 190 ; \mathbf{e}$, bilonnet allongé, $\times 130 ; \mathbf{f}$. corpuseule turriforme (d'après R. Penrient), $\times: 30$.

des batonnets recourbés el munis sur les còtés de petites denticulations dont les dimensions sont d’aillems variables e : les plus pros portent souvent sur leur cóté convexe des lubere. arrondis disposés en pelites rangées lransv.

La couleur générale est brunâtre, un peu plus claire sur la face ventr., les tuberc, de la face dors. sont bruns : la coloration persiste dans l'alcool.

L:H. sanctori se reconnaît très faeilement aux grands append, allongés de la face dor's, bien différents des pédicelles ventr., à lat présence d'un org. de Cuvier qui est toutefois moins dévelop pri que chez $\mathrm{l} H$. forskali, et enfin au pourtour tout i fait lisse des disques des corp. lurriformes.

Lill. sanctori a cté d'ahord trouvée à Naples ef elte a été retrouvée ensuite aux Açores; sur nos cotes, elle a été signalée à Arcachon et à Saint-Jean-cle-Luz. 
R. Perrier l'a étudiée sous le nom d'H. farcimen (I).

H. impatiens Fonskil II. botellus sedexid. Fig. 129. - Voir : SElexk. l×

Celle Ilolothurie, qui est presque cosmopolitr, peut alleindre de grandes dimensions lans les mers chaudes el un échant. représenté en couleur par simer avail fo cm. de long. sur une larg. de 30 à fo mm. En Médilerranée, les dimensions des échant. sont plus réduites el la longr, ne dépasse pas 15 à $16 \mathrm{~cm}$. Les lég. soul assec minces et mous, mais leur surf. est rugueuse. Les append. ambul. conservent la mème forme sur toule la surface du corps: ce sont des papilles coniques, se rélrécissanl rapidement el se terminanl par un filament cylindrique élroil : ces papilles

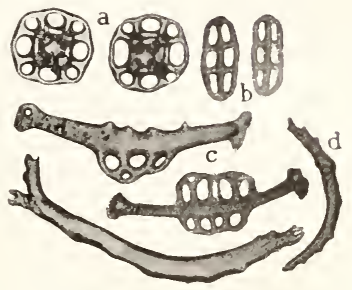

Fig. 129. - Holothuria impatiens; a. corpuseules turriformes; b, boucles: c, bitonnets dila gis; $d$, batonnet itroil, $\times 130$. sout irrégulièr. dispoxíes el ne sont pas très serrées; elles offrent souvent des alignements longit. mais qui ne se

(1) Je suis persuadé qu'une confusion s'est produite relat. à l'II. sanctori, ct que l'H. farcimen, décrite sommairenent par Selexka, puis très complèt. par R. Perher, n'est pas autre chose que l'H. sanctori. Delle Chiase, qui a créé l'espèce, a publié quelques bons dessins d'échant. provenant de Naples sur lesquels on reconnait parfaitement les pédicelles très serrès de la face ventr. et les grosses papilles coniques de la face dors. La station zoologique de Naples a mis en vente sous le non d'II. sanctori des indir. qui sont tout à fait conformes à ces dessins; j'ai constaté chez eux l'existence d'un org. de Cuvier. D'autre part, je possède dans ma collection des exempl, des Açores que j'ai reçus du Musée de Las Paimas, dont les caractères ext. et surtout les sclér. sont parfait. identiques à ceux des échant. de Naples. En particulier, le disque des corpusc. turriformes a les bords lisses, sourent un peu onduleux, mais toujours depourrus d'aspérités quelconques et il existe un org. de Cuvier. Banfors a aussi recueilli l'/l. sanctori aux Açores, et comme la détermination de ses exempl. est due à Ludwig, on peut étre certain qu'elle est correcte.

Il semble que les caractères de l'H. sanctori n'auraient fait de doute pour personne si Théel (1886, p. 22\$) n'avait dit qu'il avait reşu de Manexzeller, sous le nom H. sanctori, une Holothurie contracte provenant, paraft-il, de Naples, et chez laquelle le disque des corpuse. était épineux. Aussi Théel séparait-il de l'H. sanctori dont les corpusc, turriformes auraient le disque epineux, l' $H$. farcimen ayant des disques lisses. R. Perrier a adopté cette maniẻre de voir et il a donné le nom d'H. farcimen à des Ilolotburies recueillies par le "Travailleur " et le "Talismann", aux Açores, parce que les disques de leurs corpusc. turriformes avaient les bords lisses. Cette divergence tient uniquement à l'échantillon que Théel a reçu de Mafenzeller. Je suis persuadé que cet échant. à disque épineux, n'ètait pas une $H$. sanctori, mais bien une autre espèce : il y a eu erreur de détermination, et peut-être metme de localite. Pour moi, I'II, farcimen 
continuent pas au delit de quelques $\mathrm{cm}$. I:Il. impatiens possède un org. de Cuvier comme l'/l. forskali, mais elle est heaucoup moins sensible que cette dernière anx excitations extérieures el elle ne rejelte pas facilement ses tubes, pas plus d'ailleurs qu'elle ne rejette son intestin.

Lessclér. consistent : $1^{\circ}$ en corpuse. Lurriformes dont les disques circulaires nnt les bords lisses avee 8 orif. périphér. assez grands; les tourelles sont larges el constituées par $\mathbf{f}$ colonnettes général. réunies par une seule travée transs. a ; 2" en boucles très nombreuses, ovalaires et allongées, de dimensions général. très uniformes, présentant 3 paires d'orif: allongés et disposés régulièr. ; leur surf. est parfaitement lisse b . Les papilles renferment en outre des bitomnets de forme très variable c et un disque termin. rudimentaire.

La couleur des échant. vivants parail se conserver sans modification importante dans l'alcool; elle est d'un brun jaunâtre ou violacé plus ou moins foncé, arec des laches brunes plus foncées, irrégulièr. réparties; les papilles sont plus claires.

En lléditerranée, I'H. impatiens est assez commune dans les stations littorales à 2-i m. de prof. parmi les Algues. Je n'en connais pas d'exemplaire authentique provenant de nos eôtes de l'Atlantique; en revanche elle est extrêm. répandue dans les mers chaudes, it Amboine, aux Philippines, dans la mer liouge, ete.

H. tubulosa Guems. Fig. 130. - Voir : Marexzenler, 187', p. 314 .

Le corps peut alteindre de grandes dimensions, 25 et mème $30 \mathrm{~cm}$. de long. sur 5 à $6 \mathrm{~cm}$. de larg. Ja rég. ventr. porte de nombreux pédicelles serrés, irrégulièr. répartis ; la lace dors. présente de- tubérosités nombreuses et de grosseur différente, coniques et éparses. terminées par une petite papille allongrée. Les tég. sont très épais, asse\% corlaces, avec des sclér. de plusieurs formes. Ce sont dabord des corpuse. turriformes, très petits, dont le disque hasilaire a un contour épineux et dont la tourelle se termine par plusieurs pointes a . Les stcér. les plus nombreux sont des boncles dont la surf. présente toujours de petites aspérités enniques, pointues et assez. rapprochées. Le plus habit. ces boucles ont une forme ovale, avec 3 paires d'orif. successifs disposés srmétriquement b, mais souvent ces boucles s'allongent et oflrent de $\boldsymbol{t}$ ì 6 paires d'orif. successifs $\mathbf{c}$. Elles peuvent aussi présenter cerlaines irrégularités dans leur contour. De plus, les orif. de ces boucles pensent devenir très petits el mème faire complèt, défaut, ef l'on parse ainni is des corpuse. pleins. à surf. rugueuse et mamelomée. quion rencontre surtout dams la face ventr. C'est aussi dans cette face que les boucles sallongent le plus el le nombre de leurs orif. peut atteindre le

n'est qu'un synongue de l'H. sanctori. d'ai d’ailleurs pu eludier les exempl. du a Travailleur " el du "Talisman " et constater que c'ètaient bien des IJ. sanctori. Quant aux H. lentiginosa Manenzeler et arguinensis Kinter et VaNey, ce sont 2 espèces différentes de l'H. sanctori. 
chiffre de 12 à 15 paires $\mathbf{d}$. Les papilles dors. renferment des boucles et des corpusc. turriformes, avec des batomels allongés dont la rég. méd. est souvent élargic f. Il existe aussi des pl. allongées, munies de 7 ou 8 paires doril., e mais qui ne sont jamais aussi grandes que sur la lace ventr.: il existe d'ailleurs toutes les formes de passage entre les pl. et les bàtonnets. Les pédicelles ventr. renferment des corpusc. turriformes, des boucles, des pl. allongées et perforées ainsi que des bâtonnets. Enfin les tentac. oflrent des batonnets droits ou légèr. arqués, d’un tissu hyalin, mumis sur les bord: de petites pointes très courtes; mais à coté de ceux-ci, on rencontre des bâtonnets beaucoup plus gros dont la surl. est couverte d'aspérités très serrées, de telle sorte quïlscessent d’ètre transparents : leur lorme est souvent celle diun

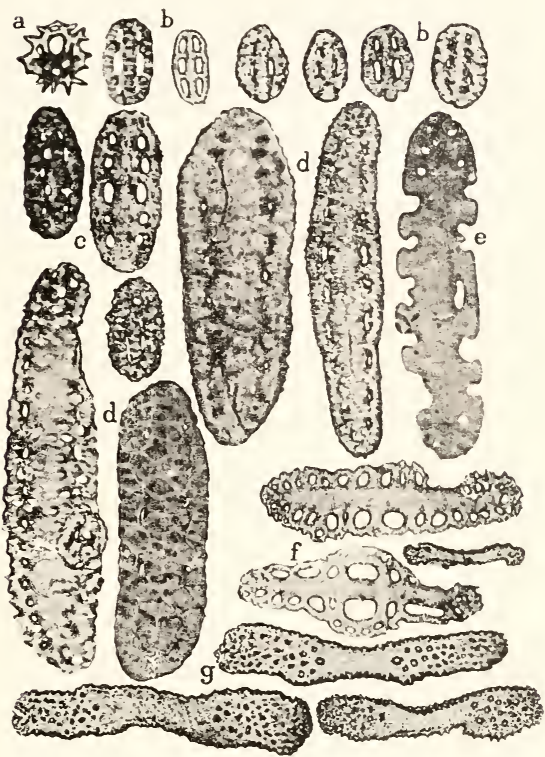

Fig. 130. - Holothuria tubulosa; a, corpuscule turriforme; b, petites boueles: c, houcles plus grandes: d, boucles tres grandes; e, plaques a contours irréguliers des papilles dorsales, X 190 ; f, bâtonnet élargi des papilles dorsales, $X 130 ; \mathbf{g}$, bâtounets des tentacules, $\times 7 \%$. biscuit très allongé, dépourvu de perlor. dans la rég. moyenne, tandis que les extrém. offrent quelques petits orif. (g) Il n'existe jamais d'org. de Cuvier.

Chez l'animal vivant la couleur est d'un brun plus ou moins foncé, marron, brun-rougeâtre ou brun-violacé sur la lace dors.; la face ventr. est beaucoup plus claire. La partie term. des append. n'est jamais blanche. La couleur change peu dans l'alcool.

Il est difficile et même impossible de conserver plus de quelques heures l' $H$. tubulosa intacte après qu'elle a été capturée. Une fois en captivité, elle rejette en effet, très rapidement ses viscères par l'anus, mais elle peut survirre quelques jours à cette ériscération.

I:H. tubulosa est une espèce essentiellement littorale qui se montre surtout en 
Méditerranée oì elle vit sur le sable, entre les touffes d'Algues et d'herbes, à quelpues dm. de prof.. et on peut la eapturer facilement avec un grappin ; sur certaines plages, elle est d'une extreme abondance. Elle descend dans les prairies de Zostères et atteint $30 \mathrm{~m}$. de prof. EHe est ordin, associée en Méditerranée, aux H. polii et forstali dout on la distingue facilement. Elle existe égal. sur nos côtes de locéan et clle a cité signalée à La lioelelle, à Biorritz, ete.

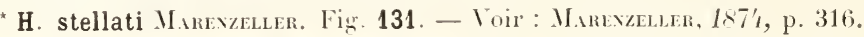
Extérieurement, le corps

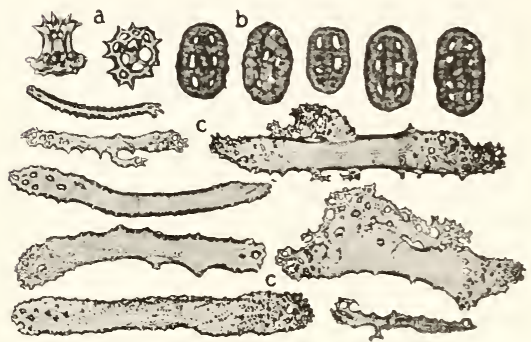

Fit. 131. - Holothuria stellati; a, corpuscule turriforme; b. boucles, $X 190$; c, batonnets divers de tentacules, $X 90$. ressemble beancoup à celui de l'Il. tubulosa, mais les lég. sont plus minces et phus mous. Les pédicelles ventr. ne sont pas très serrés el la lace dors. ollire, en phusle pédicellesidentiques a ceus de la face ventr.. quelques grosses éminences coniques, terminées par une palp. mince at allongée: ees éminences sont peu nombreuses, mais elles sont souvent disposées en rangées longit. assez apparentes, au nombre de $\mathbf{f}$ à 5 .

Il existe en outre sur les cotés du corps, a la réunion des faces dors. et ventr., une rangée très régul. d'éminences beancoup plus grosses que le autres. La couleur est d'un brun assez foncé sur la lice dors., plus claire sur la face ventr. avec des taches blanchàtres irrégulièr. distribuées.

Les sclér. sont moins variés el plus simples que chez l'/l. tubulosa. Il existe descorpusc. lurriformes a el des boucles b ayant les mèmes caract. que che\% colle dernière, mais lom nobserve jamais ces pramles boncles ou pl. allongées. spinulenses. possédant jusqu à 11, l: el meme lí paires de perfor.; ces perfor. elles-mèmes ne disparaisent jamais complet. Il est d'ailleurs rare que les boucles offrent plus de 5 pares d'orif. Les tentac. renferment des bâtomnets droits et arqués c), dont le tissu reste toujours hyalin, même dans les plus grands batonnets alteignant 0,45 à $0,5 \mathrm{~mm}$. de

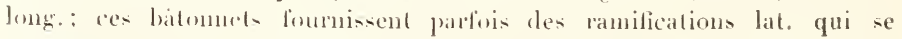
réunissent en amas volumineux domnant des formes plus compliquées que celles qu'on trouve chez l'll. tubulosa.

Je no connais pas d'échant. authentique d'H. stellati provenant de nos cotes: tous les indir. qui ont été décrits provenaient de Naples ou de l'Adriatique.

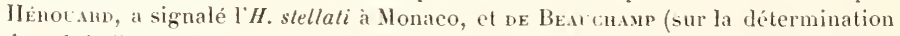
de celui-ci) à Saint-Jean-de-Luz, mais jai pu examiner les exempl. cités par ces auteurs, et j’ai constaté qu'il s'agissait simplement de l'H. Iubulosa. En somme 


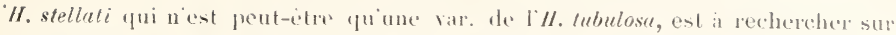
nos cotes.

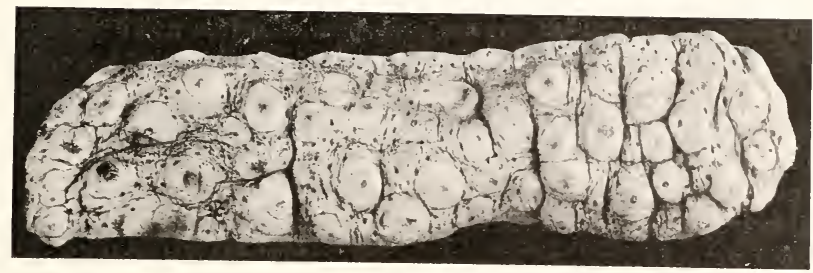

F1t. 132. - Holothuria mammatu; échantillon du . Talisman .. en alcocl; face dorsale, grandeur naturelle.

H. mammata Grebe. Fig. 132 el 133. - Voir : Ltdwlg, LAst, p. 6is; R.

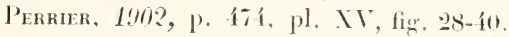

Cetle espece ne parait pas acquérir de grandes dim., le trpe de Grabr. avait tone long. de 11 cul. : cest à peu près la long. de l'indiv. que je représente ici liw. 132 . La forme générale est roisine de celle de l' $/$. tubulosa, mais la face dors. othe de très gros mamelons atteignant 7 ou 8 mm. de larg.et formant 5 à 6 rangées lougil imégul. ; ce-t à ces mamelons qui se terminent par une papille amineie que l'espèce doit son nom. La lace ventr. ninllre que des pédicelles de la forme or-

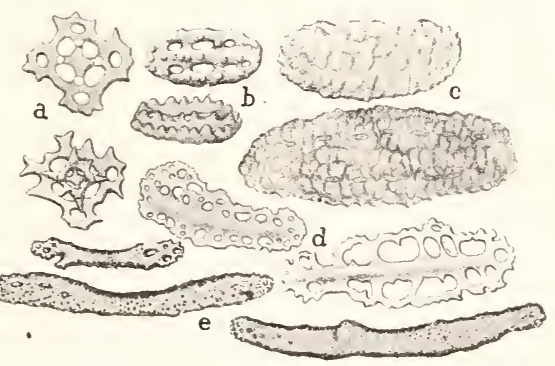

Fir. 133. - Ilolothuria mammata; a, corpuscules turriformes ; b. boucles ; c, boucles très grosses it perforations rudimentaires, $X 180: d$, plaques perforces des prapilles dorsales, $X 100$ (llapris R. PEnmer): e, hitomnets des tentacules, $X 90$. dinatre, relativ. pen nombreux et éeartis les uns des autres. Les corpuse. turriformes der leg. sont pent abondants of ressemblent a ceux de l'/l. Lubulosa 133, a . Les bundes sallongent moins que dans cette demiere espece et nollient général. que 3 pares de pertor, maipeuvent en avoir 5 ou 6 ; b : leur surface ast muniede petits tubere, ter-

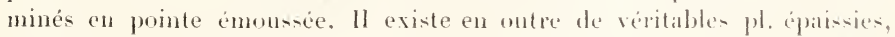
plus grandes que les boucles et dont la surf. est ribice on mamelonée c : leurs perfor. sont très réduites et parfois mime manquent complètement : on 
peut en compter jusqu'ì fo ou 8 paires. Les pédicelles ventr. et les papilles dors, renferment de grandes pl. perforées, oflranl sur les deux faces une cote médiane légèr. sillante. el, de chaque coté, de grandes perfor. plus ou moins nombreuses d ; les boucles sont plus allongées aussi que dans le lèr. général du corps. Les lentac. renfermenl des batonnels arqués qui peuvent itre aussi lougs que chez I'Il. Iubulosa, mais ils restent plus minces el ne sélargisent pour ainsi dire pas aux extrém. qui noflrent qui un petit nombre de perfor, ; dailleurs leur surf. est peu ruguense et ils restent a peu pres transparents an lien debre opaques comme che\% l'll. tubulosa e. Il existe un org, de Cuvier comprenant mue vingtaine de lubes.

La couleur des échant, vivants est d'un rouge brun foncé avec la face ventr. plus claire; elle disparail en partie dans l'alconl.

L'H. mummata a été souvent reunie a l'II. tubulosa, mats en rialite elle en est bien distincte : lapparence ext. est tout it fait ditférente et les gres mamelons de la face dors. sont trés caractéristiques: les boucles des tég. ont la surf. moins épinense et leurs dim. sont plus uniformes; enfin il existe un org. de Cuvier. earactere extrém. important pour la séparation des 2 espèces. l:II. mammata a été déconverte ì Naples; elle a été draguee i $7 t$ m., dans les parages de Bonifacio, par le "Talisman ", et Ilacmsso l'indique à Rapallo it mu m. de profondeur seulemont. Elle se reneontrera certainement sur nos cotes de la Méditerranée, dans les stitions littorales ou cotières: d'ailleurs elle est pent-etre identique à l' $/$. mamillatu que kisso signalait is Nice.

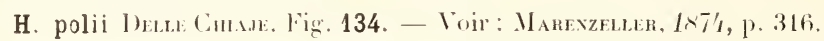

Le corps resle toujour:

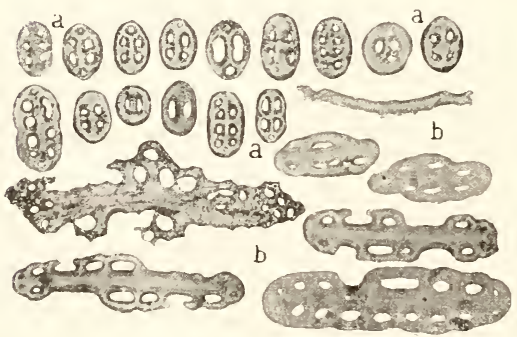

Fir. 134. - Ilolothuriu polii: a, boucles, $\times 190$; b. plarjues allongeres of bitomets de diverses Formes, $\times 130$. de grande taille mais il natleint pas tout ì fail les dimensions que lon rencontre ordin, chezl'H.tubulosa et il dépasse rarement 20 a $22 \mathrm{~cm}$. de long. sur 4 on 5 de larg. Les append. consistent surtout en tubes très serrés el très nombreux sur la lace ventr. qu'ils recourrent uniformément sans présenter d'arrangements régul.: sur lat lise dors.. ils sont moins nombremx et plus fins. Enoutre, la face dors. oftre des fuberc, eoniques, irrégulièr. disposés. toujours moins développés que chez l'/l. tubulosa. Tous ces append. pédicelles on papilles, sont terminés par une extrém. blanche qui tranche nettement sur la conlenr trè foncée, brun noiràtre, du reste du corps et donne à reflo espece un lacies bien recommaissable. 
Les tég., assez épais, renferment des sclèr. caractéristiques. Les plus abondants sont des boucles à contour ovalaire possédant ordin. 3 paires d'orif. et dont la surf. est toujours parfait. lisse (a . Certaines de ces boucles sont un peu plus allongées et offrent de 1 à 6 paires d'orifices; d'autres sont incomplèt. lormées: il leur manque une partic plus ou moins étendue de l'un des cotés, on des deux cotés, ou encore de l'une des extrém. Dans une forme assez fréquente, on observe 2 paires de perfor. symétriques et successives, puis une perfor. plus petite vers chaque extrém, de la boucle. Il existe aussi des corpusc. Lurriformes analogues à ceux de l'H. tubulosa. Les pédicelles ventr. renferment, outre les boucles et les corpusc. turriformes, des pl. allongées et assez grandes à contour irrégul., munies de perfor. nombreuses et inégales, mais dont la surf. reste toujours parfait. lisse (b. Les tentac, renferment des bâtonnets allongés et arqués, un peu amincis aux extrém., dont la surf. est tantòt lisse, tantòt hérissée de pointes, plus des pl. perforées à contour irrégul.

Après sa capture, l' $H$. polii rejette ses viscères un peu moins rapidement que II. tubulosa. l'org, de Cuvier fait complèt, défaut, et s'il a été attribué à l' $H$. polii. éest par suite d'une eonfusion avee l'H. forstiali.

Lill. polii est surlout comnune en Véditerranée : ést une espèce littorale, ordin. associèe aux H. tubulosa et forskali. Elle descend dans les prairies de Zostères. mais ne parait pas dépasser les limites de la "broundo " sur nos còtes de Provence. Elle a été signalée sur nos côtes de l'Atlantique par Barmoss (iles des Glenans); comme on l'a indiquée aux Canaries, il est possible qu'on la reneontre en divers points de nos côtes océaniques.

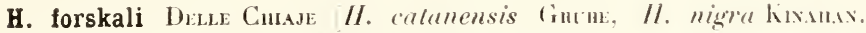
Stichopus selentice B.хrnols. Fig. 135. - Voir: Bell, $1 \times 9 ?$, p. 49. pl. VIII, fig. ; H. nigra; Kinhlen. $18 y^{\prime} 1$, p. J et 13. Cette espece a été très souvent confondue avec les $\boldsymbol{H}$. polii et impatiens. Elle rappelle la première par la coloration foncée de ses tég. el la deuxième par la présence d'un org. de Cuvier: je renvoie pour la discussion de cette synon. a mon travail de $1 \times 9 y^{\prime}$ p. o

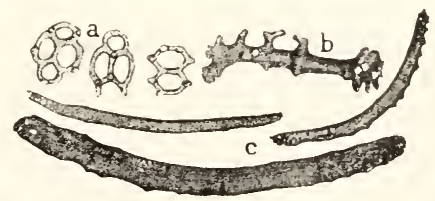

FIG. 135. - Holothuria forstiali; a, corpuseules turriformes atrophiés (d'après BELL); X 450 ; b, bâtonnets ramifiés des téguments, $\times 190 ; \mathrm{c}$. bâtonnets des tentacules, $X \div$ \% et 13).

Les dimensions sont toujours assez grandes: la long. 'atteint 20 à $25 \mathrm{~cm}$. sur 4 à $5 \mathrm{~cm}$. de larg. La régr. ventr, offre des pédicelles très serrés formant ordin. 3 rangées longit., la rangée méd. plus large que les rangres lat. Sur la face dors., les append. forment des papilles coniques se terminant en un fila ment mince. Les tégr., tout en élant asser épais, sout très mous ct facilement déformables: ils sont remarquables par la réduction considérable des sclér, 
qui sont peu abondants et surtout tris petits. Ce sont de petites pl. ollrant le plus souvent $\{$ orif. srmétriques dont la long. ne dépasse pas 10.1$)$.2 mm., el portant parfois de petits tubere. représentant des vestiges de colonnettes, tandis que la pl. elle-mème représente le disque rudimentaire d’un corpuse. turriforme a). Les pédicelles ventr. el les pap. dors posiedent des pl. analogues, toujours très peu ahondantes, anxquelles sajoutent quelques bitomets élargis, munis, sm les bords, de petits prolongements b . Iu contraire. les tentac. renferment des bitonnets allongés, legèr. arqués. i extrém. rugueuses, munis de dentionles on de petits lobes c. liorg. de Cuvier est tres développé. (Quand on eapture une //. forstiali, on remarque que les teg., tres mons, we deviement jamais rigides comme che\% les /I. tubulosa et polii. si lon tient un instant l'Jolothurie a la main en exerçant sur elle de légères presions, on la wil d'abord rejeter par l'anns des filaments hlanes, opaques, tres longs, très athésils, et qui se fixent fortement sur les corps étrangers: ce sont des tubes de Cuvier. Lexpulsion du tube diw. ne vient que plus tardivement.

A l'état vivant, l'/l. forslialia toujours une couleur très foncée, du moins en Méditerranée el chez les échant. littoraux la face dors. est noire aree lextrém. des papilles blanche et la face ventr. un peu plus claire. Les indis. provenant d'une certaine prof. ont une coloration moins foncée: la face cors est d'un brum plus ou moins clair, et la face ventr. est brum jaunitre su mème tout a fint jamne. Ansi que je l'ai signalé il y a fort longtemps, Ix!́', le pignent qui colore les tég. de l'll. forstiali est en partie soluble dan- l'alcool et lui communique une très belle fluorescence rerte, mais la coloration de limimal n'est guère modifiée.

Lill. Forstiali ent tris eommune dans toute la Vediteranee ainsi que sur nos cotes allantiques. En Méditerranée, efte est tress abondante dans les pratries de \%osteres et peut remonter jus ju it 3 on \& m. de prof., tandis quelle pent descendre jusfü: $: 30$ m. sur nos cotes de Provence, elle est tries commune en broundo ". ol alleint minc les foncls coralligènes. lille est égal. assez répandue sur nos cotes de l Mllantinge et a sté signatée dans plusieurs localites des cotes l' Ingrleterre, entre o el in m. de prof.

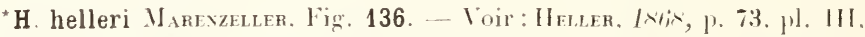

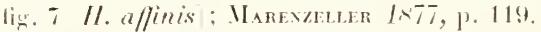

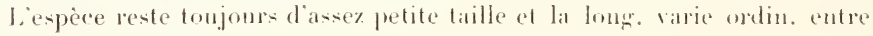
10 et 20 mm.; elle peut cependant atteindre $55 \mathrm{~mm}$. Les petits échant. rappellent une (ucumaria: le corps est pentagonal, les tég. sont résistants et rudes, el les tubes ambul. sont disposés en í rangées subégrales: sur les indiv, plus grands, les lég. sont plus mons, les pédicelles de la face dors. ont la forme de papilles, taudis que sur la lace ventr., ils gardent la lorme de tubes.

Les sclér. consistent surtont en corpure. Lurriformes dont le disque

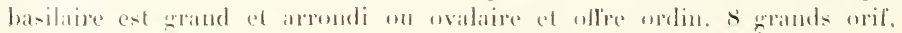


périphér. ef quelpues autres beanconp' plus pelits an plus de f orif, centr. Les tourelles sont très allongres of étroites, el leur haut. égale le diam. dn disque: leurs travées transv. sont an nombre de 3 à 5 (a). 11 existe en plus des boncles olliant habil. 3 paires d'orif., a surf. rnguense (b). l'arlotis ces bomeles sallongent el le momber de leurs orif. peut alleindre le chillie de 10 ef mème de 12 paires (c. Les pédicelles ventr. el les tentac. renferment des bàtomnets recourbés (d) plus ou moins ypinuleux.

L'animal vivant est d'un brun assey foncé tacheté de blane, la face veutr.

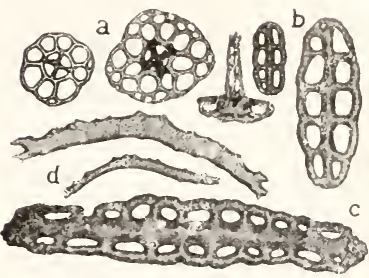

Fig. 136. - Holothuria helleri; a, corpuscule turriforme, $\times 130 ; \mathrm{b}$, boucles; c, tres gratule boucle,

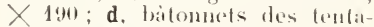
cules, $\times 130$. est plus foncée; les extrém. des parpilles dors. sont ordin. plus elaires el celles des pédicelles ventr. sout jaunes: les tentac. sont d'm jaune asser vil.

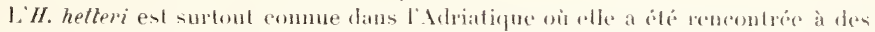
pof. de 2 a 3 m. senlement, parni les Algues. Cetle esjece na pas eneore étí signalée sur nos côtes de lrance, mais je possède dans ma collection un fehant. provenant de Bône: on la rencontrera certainement dans d'antres localités de nos côtes d'Algéric et de Tunisie on peut-itre de Provence.

\section{(i. STICHOPUS BHANDT.}

Aspidochirote possédant is it 20 tentac.; les append. anubul. se présentent sous forme de pédicelles sur la face veutr. où ils sont disposés général. en 3 rangées longit. plus ou moins distinctes, et sur la face dor's, sous forme de papilles; les tubes génit. aont groupés en 2) faisceaux, un de chaque coté du mésentère dors.

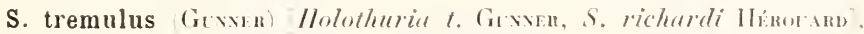

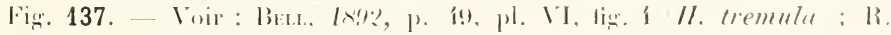

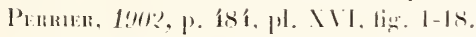

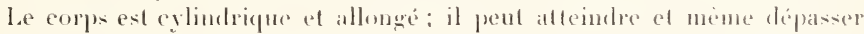

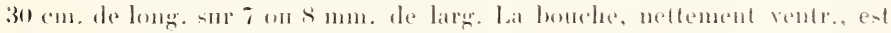
silue a 1 ou $2 \mathrm{~cm}$. en arriere de l'extrem. ant. et on distingne antonr delle une couronne de papilles disposées plus ou moins régulior. en ㄹ cereles. I a face dors. offre des papilles pen nombrenses, espacées el terminées par tme extrém. pointue. Les selér. des tég., très abondants, sont de 3 sorles: ce sont d'abord des corpuse. Iurriformes bien développés, itrec th disque asse\% irrégulièr. circulaire, dont le bord est hérissé de dents aigü̈s; el une fomrelle formée de 1 colonnettes reliées par 2 étages de travées transv. (a). On tronve, en outre, des corpusc. grêles et épineux, plus ou moins ramifiés, dont les bras 
étroits portent sur toute leur long. des pointes aiguës; les plus simples sont arqués ou ont la forme d'un $\mathrm{I}$ ou d'un $\mathrm{Y}$ b', puis ils arrivent, par

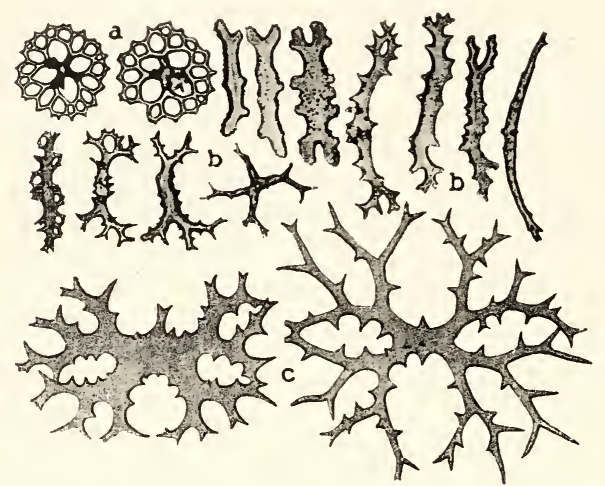

Fig. 137. - Stichopus tremulus; a, eorpuseules turriformes, $\times 130 ; \mathrm{b}$, hatonuets diver's, $X 70$; c, bâtonnets très ramifies (rlapres R. Pemurn), $\times 180$. ramifications successives, à des formes plus compliquées c. Très souvent ces corpusc. s'élargissent mais en même temps ils $\therefore$ aplatissent en forme de lamelles épineuses. Les papilles dors, et les pédicelles ventr. renferment les mêmes corpusc.; les tentac. offrent, en outre, des corpuse. turriformes.

La couleur générale est d'un rouge plus ou moins vif sur la face dors., rosée ou blanchâtre sur la face ventr.; cette dernière

oflre souvent de petites taches très foncées. Les échant. en alcool sont complèt. décolorés.

Le St. tremulus est tris commun sur nos cotes de l'Atlantique, a partir de $80 \mathrm{~m}$. de prof.; il n'est pas connu en Véliterrante. 11 remonte dans les mers du $\mathrm{N}$. sur les côtes des lles Britamiques êt de Norvège ot se retrouve égal. au large des côtes d'Espagne.

En captivité, le St. tremulus ne rejette pas ses viscères et il peut virre plusieurs jours intaet: mais an bout d'un certain temps, ses tég. subissent une modification profonde consistant en une sorte de fonte ou de liquéfaction de leur couche superficielle.

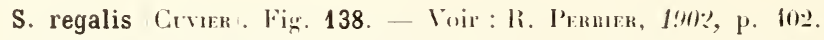

l.e rorps peut atteindre de grandes dimensions of la long. arrive souvent a $30 \mathrm{~cm}$., mais les indiv. de 20 a $2.5 \mathrm{~cm}$. sont les phus lréquents ; la larg., qui est en moyenne de 6 a 7 cm.. se conserve sur presque toute la long. du corps. Celui-ci est plus ou moins aplati, surtout sur la face ventr. ; la face dors. est convexe. Les bords amincis du corps sont garnis de grosses papilles se continuant sans interruption sur l'extrém. ant., en avant de la bouche qui est ventr. et est entourée par un cercle incomplet de papilles.

La face ventr. offre 3 rangées plus ou moins distinctes de pédicelles et la face dors. porte de gros tuberc. terminés chacun par une papille conique et pointue : ces tubere.. assez rapprochés, forment des rangées longit. plus ou 
moirs régul. La couleur des tég., à l'élat virant, est géućral, jamm hrunitre et devient plus claire sur la face ventr. La face dors. présente soment de grandes taches blanches arrondies ou ovalaires asse\% rapprochese les unes des autres: parfois la coloration passe an rose plus ou moins vif : cette deruicre rouleur disparait complet. dams l'alcool, tandis que les teintes brumes on jannes restent à peu près intartes.

lessclér. destég. consistent principal. en corpuse. furriformes asse\% grands. dont les colonnettes sont rémies par 3 étages de travées transs. el quelquefois mème par 1 ou ja les extrém. de ees colomnettes sont munies de pointes aiguës qui traversentle lég. el le rendent tres rugueux b. 11 existe an antre les bàtomets allongés el aplatlis. droits ou reeourbés, sélargissant anx extrém. el souvent aussi en leur milieu, et offrant dans ces rég. élarquies des perfor. ordin. asse\% grandes: les borls sont munis de spinules a loes

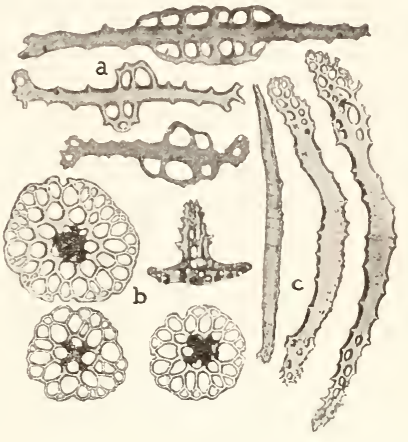

Fis. 138. - Stichopus regalis; a, biitomnets à région moxenne élargie; b. corpuscules turriformes, $X\{30$; c, bitonnets allongés, $X 71$. tentac. renferment des batommet- de taille el de long. variable, mais qui restent toujours étroits : les plus petits sont lisses landis que les autres sont hérissés de pointes coniquesasse\% fortes c .

Cette espece pent vivre assez longtempen en caplivité sans expulser ses viscires: le rejet cependant se produit au hont d'un certain temps.

Le St. regalis est surtout comm en Méditerranér oì il rit it des prot. variant de quelques m., à $30 \mathrm{~m}$. el au delit; sur nos côtes de Provence, on le rencontre de préférence dans les fonds vaseux de la "lnoundo " oì il est associé à l'Échinns acutus; les pecheurs lappellent " langue de chat ". Wans l'Atlantique, il parait très abondant ì partir de 30 ou $40 \mathrm{~m}$., mais il peut descendre juscun à plusiens centaines de m. et il se montre alors sousent associé au s'. tremulus. 


\section{S. Cl. HOLO'THI RIES IPOIESS}

\section{F、MOLPADIDÆ J. MüLLER}

IIolothures apodes c. à d. nianant pas d'aulres append. que les tentac. qui sont an nombre de $1 \%$, simples on digilés; il existe des org. arborescents mais pas d'org. sensorjels sur les lentac., ni d’entomoirs ciliés sur les mésentères. Les sclér. comprenuent des corpusc. Lnrrif., des bàtonnets, des pl. perforées, des ancres, et souvent des corpuse. colorés renfermant du phosphate de fer; il existe des canaux aquifères raul.

\section{G. MOLPAdia Cuvier Ankyrodermu Dixmlssix et Konex].}

Les fentare sont pen on pas ramifies : le oor ps se refrecil brusquenent dans sa reg. post. de manièr it former me sorte de quene. les sclér. nombreux consistent surtout en pl. perforées el en bitrmels fusiformes, accompagnés d'ancres assez rares, et de nombreux corpuse, phosphatipues d'un rouge vineux.

M. musculus Risso Haplodactyla mediterranea Gras, Inliyroderma musculus auct.]. Fig. 139 et 140. - Voir : L. Cuari, 16117, p. 16.5, pl. XII.

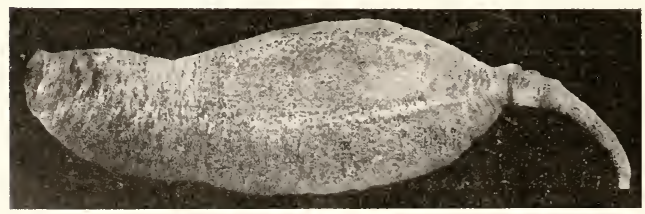

Frn, 139 - Holpadin musculus; vor lalimele. ligèremenl grosice.

Les lentac. sont courts, it peu près ansi longer yue latres of lemr extrim. prosente de rhatgue erole 111 ramean élargi. Les leg. sont épats el ruguem: il ny a pas la moindre indicationd'append.

ambul. antres que les lis tentae. Iess selér. consistent surtout en bâtomnets allongés, lusiformes, dont la partie métiane élaryie en percée d'orif, ordin. 
inégaux et souvent au nombre de $\mathbf{i}$ fig. 140, a). Le centre de la partie élargie porte parfois une petite tige dont l'extrém. est arrondie ou parfois bifurquée. On trouve, en outre, des pl. perforées, avee de nombreux orif. très grands et circulaires, de la rég. centrale desquels śetevent 3 colonnettes convergentes se réunisant en une tige vertuale termines par quelques denticulations b. A cóté de ces sclér., on trouve ausit, mais plus rarement, des pl. circulaires perforées et du centre desquelles s'élève ume longue lige terminée par quelques crochets, habil. an nombre de 6 et dont la pointe recourbée est dirigée vers la pl. d. Une autre forme. très caractéristique, esl constiluée par depl. en forme de spatules, perforées, et associées au nombre de 6 à 8 en une sorte de rosetle: 'au centre de eelle-ci se trouve une petite pl. arrondie de laquelle s'élève une ancre, Enlin, les tég. renfement

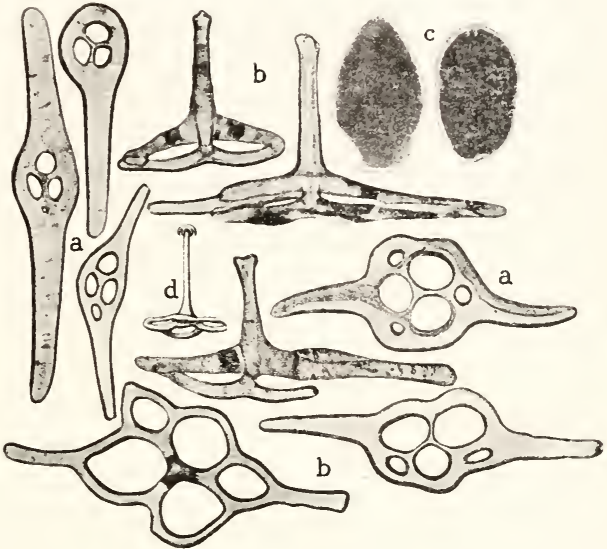

Fie. 140. - Molpadia musculus: a, liverses formes de plarpes perforeses $b$, placpues à tourelle allongie (rues de fice et de profil); c. corpuscule vineux,

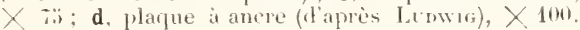
un nombre considé-

rable de corpuse. phosphatiques, sphériques on ellipsö̈danx, formés de conches concentrigues tres distinetes et colorés en rouge vineux c :

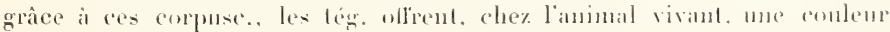

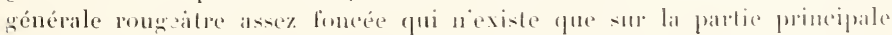

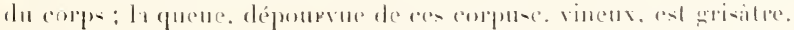

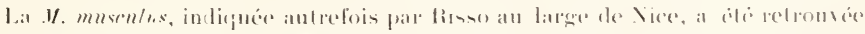

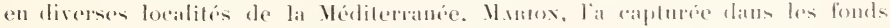

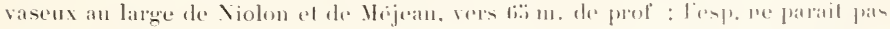

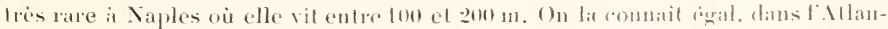
tique oì elle atteint des prof. beaucoup plus grandes : je lai capture it som 11 . de prof., par 'to longit. W., el t'to latil. N. 


\section{F. SYNAPTIDE BCrMEISTER.}

Holothuries apodes chez lesquelles l'appareil aquifire est tries réduit; il existe seulement des tentac. péribuccaux dépourvus d’ampoules, et les canaux aquilères rad. font complèt. défaut, ainsi que les org. arborescents. Le corps est eyllindrique, allongé, vermiforme: les sexes sont souvent réunis; il existe des org. sensoriels ¿ la base des tentac. et des entomnoirs (on urnes) cilies, sur les mésentères. les sclér, eonsistent an ancres articulées sur des pl. spéciales dites pl. anchorales, mais il nexiste ni corpuse. turriformes ni corpuse, phosphatiques colorés.

Les 5 esp. françaises, autrefois du g. Synapta, vivent dans le sable ou le sable vaseux : on les trouve sur nos côtes de l'Atlantique à mer basse, enfoncées dans le sable oi clles creusent une galerie enduite de mucus, et leur présence est souvent révélée par de petits monticules; elles peuvent descendre à une certaine profi., ct quatre se reneontrent égal. en Méditerranéc. Iorsque les Synaptes sont gardées en captivití, elles ne tardent pas ì se morecter en fragments : le corps se contracte de distance en distance it partir de lextrém. post. et il prend une forme en chapelet, puis les fragments succesvits se scparent les uns des autres et ne tardent pas à périr.

\section{G. LEPTOSYNAPTA Verrul.}

Les tentac, au nomb. de 10 à 13 , sont pimnés, c. à d. portent de chaque côté. "艹 à $x$ ramific. lat. simples, et ils se terminent par un lobe impair ; pl, anchorales ovalaires.

L. galliennei Herapatul). Fig. 141. - Voir : L. Clark, 1907, p. 91 : Cuḱnot, 1912 p. 62.

Le corps evlindrique atleint ordin. une long, de 122 a $25 \mathrm{~cm}$. parfois même de $30 \mathrm{~cm}$., sur un diam. de 6 à $9 \mathrm{~mm}$. Les tég., translucides, sont rosés et piquetés de taches blanches qui correspondent aux ancres d'assez grandes dim. Lestentac., au nomb. de 12, portent sur leur face buce. une douzaine d'org. sensoriels chacum.

Les pl. anchorales et les aneres atteignent d'assez grandes dim. : c'est pourquoi cette esp. adhère fortement aux doigts. Les ancres ont une long. de 0,45 a $0,50 \mathrm{~mm}$. et les pl. correspondantes $0,35 \mathrm{~mm}$. Les pl. sont ovales avec une extrém. amincie et pointue sur laquelle s’insère l'ancre, séparée du reste de la pl. par un relief plus ou moins saillant a \% La partie principale présente un certain nombre d'orif. disposés général. d'une manière régul. autour d'un orif. centr, : on trouve souvent 7 de ces orif. périphér., quelquefois plus, et, en dehors de cenx-ci, quelques petits troussupplémentaires. l.es grands orif. sont denticules sur la plus grande partie de leurs bords ; et les bord- des pl. eux-memes sont munis de petiles dents lat., coniques, en nombre viriable, mais qui śétendent rarement sur toute la périph. de la pl. 11 existe en outre, dans les tég., de petits bâtonnets en forme d'un C épaissi aux extrém. 
Les org. int. de la $L$. galliennei offrent certaines dispositions très caraeléristiques. I la suite de l'asophage, l'intestin, rallaché a l'interrad. dors. par un mésentère, continue son trajel d'abord en ligne droite, puis il se reeombe sur lui-mème pour revenir vers la bouche dans um trajet récurrent, it la suite duquel il se replie de nomvean en mème lomps qüil se rapperobe du coité ventr. Ju comps: de là il continue son trajet en linge droile jusqu’à l'extrém. post. où se trouve l'anus. Les urnes ciliées ne sont développées que sur le mésentère šétendant le long de l'interrad. dors. gauche on elles forment une rangée lomgit. mique mais bien apparente.

La L. galliennei esl tris répan-

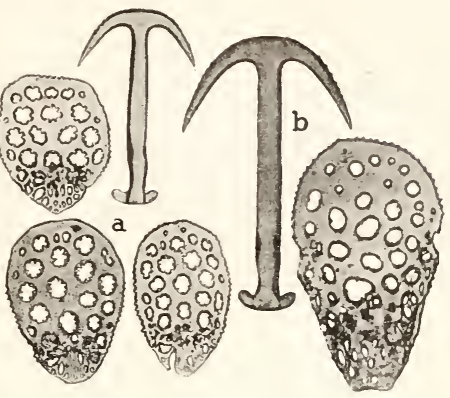

Lu. 141. - Leptosynaptu galliennei; a. plapues anchorales de taille ordinaire of une ancre correspondante; b, plaque anchorale plus grande et ancre correspondante, $\times 130$. due sur nos coles occilentales, mais elle a fité souvent confondue arec les $L$. inhærens et Labidoplax digitata; aussi il est assez difficile d'indiquer exactement son extension géographique; en tous ras,

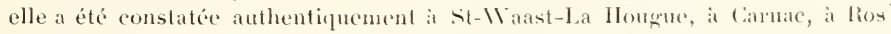
coff et à Arcachon; daus certaines localités elle exchut complìt. la $L$. inkerrens. Elle doit remonter assez hant dans les mors du Y.. et, sous le nom de L. bergensis, elle a élé signalée a Borgen, a Tromblhom, aux iles fanoë, aux llóbrieles, ete. On la reneontre à la cole, à mer hasse on it une très faible prot.

Lonwa a décrit sous le nom de L. malirankyra une syople de Yaples, muarquable par les dim, consid. de ses aneres qui peuvent atteindre 0,8 mm. de long.

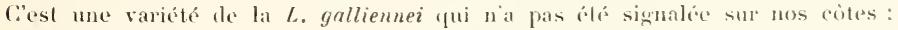
cependant je trome, dans certains échant. de binarl, des ancres et des pl. anchorales 2 fois plus grandes que dhabitude b el qui pouraient hien apparterir it lat var. makrankiyra.

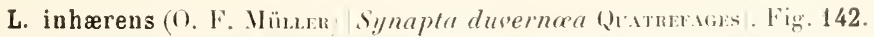

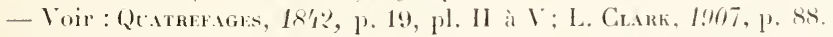

La long. du corps peut allemelre 20 on $25 \mathrm{em}$., mais ne déparae pas ordin. 10 a 15 em. et reste plus potite que chez la $L$. galliemnei. La lég. Irans-

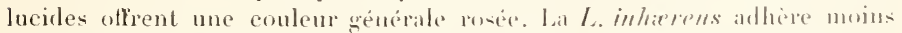
fortement aux doigts que lat L. gallienmei, et plus fortement que les Labido-

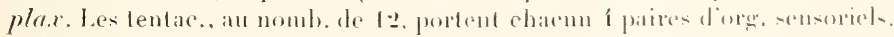

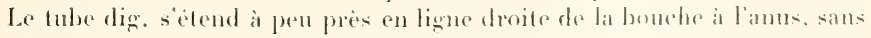
offrir eette boucle si rurieuse pui exisle ehe\% la L. galliemnei : il fial simple-

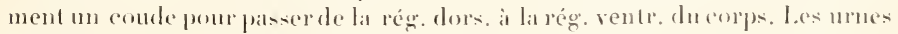
ciliées forment 3 rangr. Iongit. Les ancres sonl relativ petiles; les pl. anchor 
lates ovalaides ont des bords lisses et offrent le plus soment 7 grandes perfor, : une centr. et tipériphér. avecquelques autres beaucoup plus petites. Les grandes perfor. sont munies de fortes

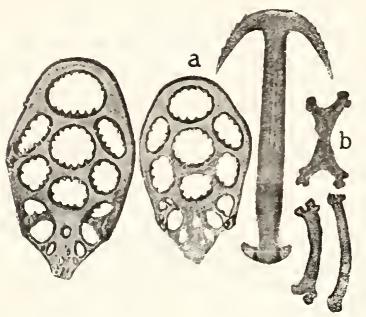

Fic. 142. - Leptosynapta inhrerens; a, placpues anchorales of ancre, $\times 130 ; \mathrm{b}$, bitonnets des tentacules, $\times 190$. dentic. qui n'existent pas toujours sur tout le pourtour des orif.; la partie pointuè niest pas séparée dn reste de la pl. par un relief a. Les tentac. renferment des bâtomnels b légèr. recourbés et olfrant ì leurs extrém. une ou 2 petites ramifications.

La $L$. inherens est très répandue sur nos côtes de l'Oeéan et de la Manche, du moins si l'on en juge par les indications des anteurs, mais il est très prossiblequ'elle ait été parfois confondue arec la $L$. galliennei. Sa présence a b́fé constatée d'une manièrécertaine à Dinard, a St-Mialo, à Roscoff, à Concarneau, à Areachon ete. On la trouve à mer basse, dans le sable vascux, mais elle peut deseendre daus l:1lautique comme en Véditerra-

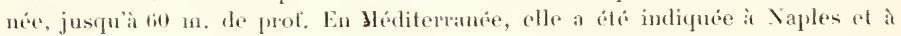
Trieste. Elle remonte jusquaux eôtes de Norvige el peut descendre jusqu ì l'embouchure du Congo. Enfin on la retroure aux États- Ínis.

Les $L$. inhrerens et galliennei sont les 2 scules espèces de Synaptes de nos côtes dont les tentac. sont pinnés. On les distinguera très facilement: la $L$. inhrerens adhère peu aux doigts, ses pl. anchorales ont les bords lisses et les bâtonnets des tent. sont ramifiés anx extrém., tandis que lia L. galliennei adhère fortement aux doigts, ses pl. anclorr, ont les bords dentic. et les battonnets des tentac. sont simples.

\section{G. LABIDOplaX Östercinex.}

Tentac, digitis, au uomls, de 11 i 12 , olfrant 3 ou f tigitations: fl, anchorales en forme de raquettes.

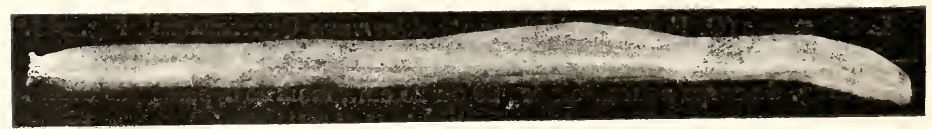

Fur. 143. - Labidoplax digitato; ve de coti. X 12.

L. digitata Moxmar Fig. 143 a 144. - Voil : L. Ci.nki, 1907, p. 9.); (ixist, 1!11:, p. 7í.

La long. alleint sour. $30 \mathrm{~cm}$. sur une lary. de 8 it 9 mm. en movenue. les fentac., an nombre de 12, ne portent ordin. vers lextrém., que 2 paires de 
pinnules lat. qui ne sont pas tout a fait sur le mème plan; il u'y a pas de pinmule termin. on bien celle-ci est rudimentaire; la face int. des tentac. offre 2 groupes longit. d'org. sensoriels, an nomb. de 12 i 15 de chaque coité.

les pl. anchorales sont allongées, en forme de rapuetle areo mu manche assez long b : la partic principale de la pl. est ovalaire, les bords sont lisices el les perfor. ellesmèmes sont loujours déponrvos de denticules. (hn abserve tres sourent í grandes perfor. di-posées en eroix, subégales, accompagnées d'autres plus pelites. Les pl. sout relat. plus courtes dans la rég. ant.du corps c el leur long. augmente dans la rég. post, ou elles ont environ $0,3 \mathrm{~mm}$.: elles sont plus larges en arrière quien avint. Les ancres correspondant it ces pl. ont une long. de 0,3 a $19,3,5$ et leurs bras sont assez divergent a, c et d, mais elles ne sont pits très longues relativ a la long. de la plaque anchorale. Le tube dig. s'étend presque en ligne droite. et, comme chez la L. inhierens, il fait simplement un eoude brusque pour pasier du coté dor's. au coté ventr. du corps: les urnes ciliées, petites et nombreuses, forment 3 rangées distinctes.

Certains échant. offrent des ancres géantes qui se trouvent très régulièr. disposées à la suite les unes des autres el suivant

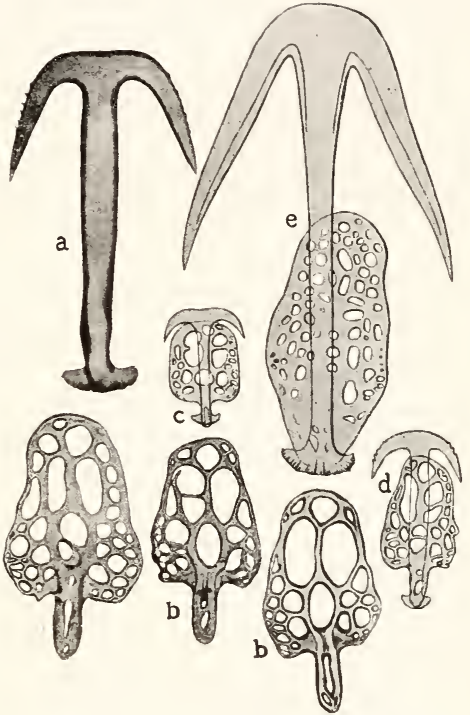

Fig. 144. - Labidoplax digitata; a, incre: b. plaque anchorale, $X 130$; c, plaque anchorale et ancre de la région antérieure; $\mathbf{d}$, playue anchorale et ancre vers le quart antérieur; e, ancre géante el sa plaque correspondante des interradius latéro-dorsaux (d'après Woopward et B.trkett), $\times 80$. une seule file, dans cluaque

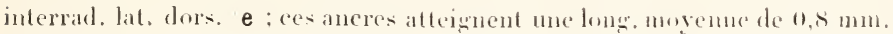
et meme $1,9 \mathrm{~mm}$, on les recomnat done facilement a lieil nu on a la louje; leurs bords sont lisses; les pl. correspondantes sont relat. petites et étroites, elles ne mesurent pas plus de 0,3 i $0,4 \mathrm{~mm}$. delong. et les perfor. nombreuses sont irrézulièr, disposées. Celte forme peut se rencontrer sur nos coiles de Bretagne et je considère les indiv possédant ces aneres géantes comme une var. distincte : il doit y avoir, chez la L. digitata, une forme matirantiyra, i ancres très développées, comme il en existe une chez la Leplosynapta galliennei. 
Che\% lamimal viant, la face dors. est fortenent colorée en rouge ou en brun vineux par de petites taches très rapprochées, et la face ventr. est blanche ou blanc-rose.

La $L$. digitala parait très répandue sur nos cóles de la Manche et de l'Oeéan: elle est souvent associéc à la L. galliennei. Elle se tronve surtout dans les stations littorales qui décomrent anx grantes marés. Elle vit aussi en Véditerranée et a dé signaléc a Marseille, à Naples, i Trieste; elle peut descendre jusquă $50 \mathrm{~m}$. de prot.

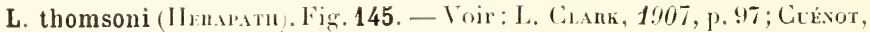
191 ?, p. 75 Synapla digitala var. thomsoni.

Celle espèce. survent confondue avee la $L$. digitala, a les mêmes caractères ext., mais elle śen écarte par ses corpusc. calcaires. Chez la L. thomsoni, en effet, il y a une diflérence tres marquée entre les pl. anchorales de la rég. ant. el celles des rég. moyenne et post. du corprs. Daus la rég. ant.,

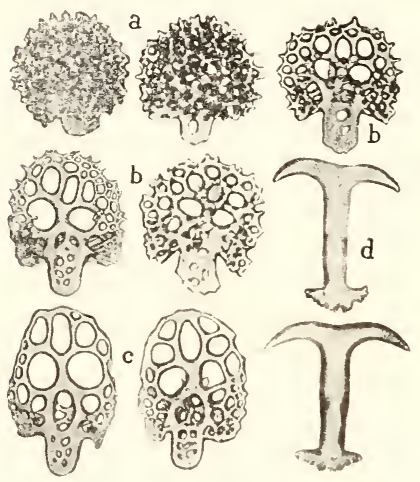

Fis. 145. - Labidoplar thomsoni; a. plaques anchorales de l'extrémité antérieure du corps; b, playqes anchorales vers le premier quart; c, plaques anchorales des reigions movenne al postérienre du corps: d. ancres corre-pumbint is ces plaques. $\times 130$. leur contour est arrondi mais offre des dents nombreuses, fortes et inég.; de plus leur tissu calcaire, au lien de compremble me seule lame mince munie de perfor. bien distinctes. porte un résean second. formé de trabécules nombrenses, tres serrées el irregulier. disposées, cachant les uril. sous-jacents a ; aussi cespl., assez compactes, se montrentelles opaques et presque noires, saul leur manche très court, qui est transparent: le diam. moyen est de $0,15 \mathrm{~mm}$. Les ancres correspondantes sont courtes et leur long. égale à peu près le diam. de la pl.; leurs bras, très fortement divergents, sont placés presque sur le prolongement l'un de l'autre. A mesure qu'on stavance vers la rég. moveme du corps. on voit le reseau second. disparatre progress. b el les pl. oflrent dies lors 5 à $f$ oril. pincipaux asse\% régulièr. disposén avee d'autres beaucoup plus petits: les bords restent encore numis de quelques dentientes; en meme temps, les pl. sallomgent quelque pen et devienuent ovales: leur long. alteint $0,20 \mathrm{~mm}$. sur 10.1 í de larg. c : les denticules penvent faire complet. déf. mais le contour reste ordin. un peu anguleux; le manche est ćpais el plus court que chez la $L$. digitata. 
On voit done qưil est nécessaire, pour séparer la $L$. digitata de la $L$. thomsoni, d'étudier les pl. anchorales dans dillérentes rég. du corps à partir de l'extrém. ant.

La $L$. thomsoni doit coexister dans beaucoup de localités de nos cotes avee la L. digitata : Bunous, à Concarnan, et Cíxor, à Areachon, ont trouvé les 2 esp. virant còte à cote; elle a cité indiquice a Naples et dans l'Mdriatigue, mais il est certain quelle a éte somvent confondue aver la $L$. digitala.

\section{(i. RHABDOMOLGUS KEFERSTEIN.}

Les tentac. au nombre de dix seulement ont les bords lestomif's; il n'y a pas de sclér. dans les tég. et les urnes ciliées font également défaut; un tube hydrophore et ure vésicule de Poli. Taille très petite.

R. ruber lifensteix, Fig. 146. - Voir : liefensten, 1862, p. 34, pl. XI, tig. 30; Lсxwme, 1!10.5, p. 458.

La long. ne dépasse pas $10 \mathrm{~mm}$. sur une largeur de 1,5 mm. environ et la couleur chez l'animal vivant est d'un rouge vif. In seul exemplaire avait èté trouvé autrefois par liefrestrix à st- Waast, et il a élé considéré par plusieurs zoologistes comme une jeune synapte, mais plusieurs échantillons ont été retrouvés à Ilelgoland dans le sable par Lıwwig, qui a confirmé les caractères indiqués par Kefressten.

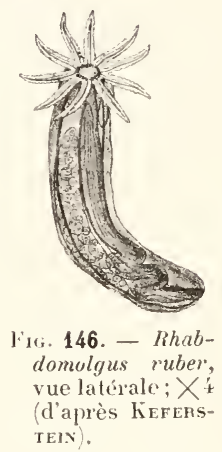

\section{CRINOIIDES}

Pour avoir une idée exacte el complète de la structure d'un Crinoỉde, i] faul considérer non pas l'une des quelques esp. vivant sur nos côtes, mais des formes telles que les Pentacrinus qui pexistent au large de Rochefort vers 1.500 m. de prof., ou les Rhizocrinus des cotes de Norvège.

Ces Crinoïdes sont rattachés au sol sous-marin à l'aide d'une longue tige formée d'art. successifs, et leur corps proprement dit, apjecí calices, est de 
petites dim.; il a la forme d'une eoupe oud'un cone, dont le sommet est fixé

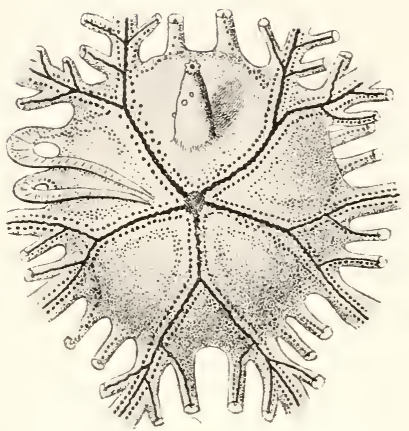

Fiti. 147. - Fice orale diune Comatule, $X i$, voe sehrimatique (d'apres Cuiм(T).

squel. : les pl. bas. et rad. passent a l'intérieur de bi coupe el ne sont plus visibtes a l'extérieur : les parois du calice sont dès lors formées par les premieres pièces brach.: lart. prox. du pédoncule, resté adhérent au calice constitue la pièce appelée improprement centrodorsale ehez ces formes. On trouve d'aborls séries de pl. suceessives, an nombre de 2 dans le $\ldots$. Intedon, et de 3 dans le 2. Leptomella, les radiales on primibrachiales, dont la dermiere ou axillaire. de forme lriangulare, porte le -2 has d'me mente paire formes par des picees suecessives dites

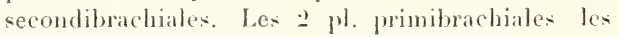
et les 3 on í premières secomdibrachiales forment parois du ealice et cest an deli que les 10 bras deviennent libres. Les articulations des pl. brach. permettent des mouvements élendus: ees articulations sont asse\% compliquees of obliques. Mais il existe de distance en distance certaines articulations spéciales. dites syzlygies, dépourvues de muscles el qui sont des lienx de moindre risistance: cest toujours a leur niveau que les hras se brisent; ces syrgies sont perpendic a la direction des bras, au lieu d'etre obliques comme les articulations pr. dites.

La base du cone anquel jai comparé le calice de nos Comalules représente la lace orale: elle est formée par une membrane renfermant 
quelques spicules et au centre de laquelle s'ourre la bouche; dans un interrad. se trouve l'anus porté par un lube saillant tig. 147). De la bouche partent 5 sillons rad. yui se bilurquent ef se continuent sur la face wrale de chaque bras: les bordi de ces sillons portent lestubes ambul. dépourwo de ventouse el disposés par grouper de 3. Lin dehor's des silloms, on remarque de nombreuses petiles spliérules enfonées dans les lég., les saccules. Chaque art. brach. porte me petite ramification lat. appelée pimnule, et éest dans ces pimules que se développent, à un moment donné, les glandes génit. (fig. 149, 150 et 151 ; les sillons ambul. se continuent sur leur face orale. Enfin, la pl. c.-dors. porte un eertain nombre d'append. appelés cirres lormes par des art. suece-sifs, dont le dernier a la lorme d'une griffe, et qui se fixent sur les corps etrangers : ils peurent servir i la locomotion. Il n'existe pas de pl. madrép. : les tubes hydrophores, qui sont nombreux, sourrent isolément au dehors.

La ponte a lieu par éclatement des parois des pinmules. Les auf's lécondés domnent naissance ì une larve libre, ovoüde, nunie de bandes ciliées, el qui ne tarde pas a se fixer par son lobe préoral; celui-ci s'allonge en un petil pédoncule supportant le calice terminé par les bras tig. 148. Puis, à un certain moment, le pédoncule se brise entre le $1^{\mathrm{er}}$ article et le $2^{\text {mo }}$ et ce premier article deriendra la c--dors. La Comatule devenue libre n'aura plus quà grossir.

Les Comatules virent général. par troupes sur les rochers, parmi les Algues, fixées a l'aide de leurs cirres: elles restent habit. immobiles les bras élalés horizont. et les relèvent lorsquion les inquiète ; ces loras peurent s’écarter, se rapprocher, śenrouler en spirale ou s'étendre en ligne droite; ils se brisent facilement, surtout lorsquon les irrite, et la rupture a toujours lieu au niveau d’une syzygie. Les bras brisés se régénèrent assez rapidement. On a constaté parfois une éviscération comparable à celle qui est frécquente chez les Holothuries : dans cerlaines circonstances mal comues, les Comatules rejettent, et cela sins périr, fous les org. contenus dans leur calice et les régénèrent ensuite en quelques semaines. Les Crinoüdes portent sourent sur leurs bras ou leurs disques des parasites particuliers appelé Myostomes.

On combit sur les coles de france í especes diflérentes de Comatules: ces espèces étaient autrefois rangées dans le sent grenre fmledon $=$ Coma-

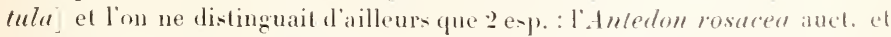

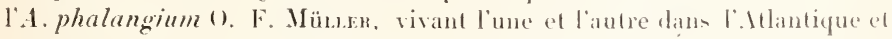
la Néditerranée; on recomnaissait cependant que les formes atlantiques étaient un peu différentes de celles de la Méditerranée. Austin Cisnok a sépare dans chacune de ces especes une forme atlantique el une lome méditerranéenne, el il a eréé mu s.. nomvean, leg. Leplomelra pour l’.1. phalangium de la Méditerranee et la forme correspondante de l'M lanlique ; l'ancien g. Anledon renferne I'A. bifida de l'. Mllantique et l'A. mediterranea de la Méditerranée. De plus ce meme antenr ennidere que

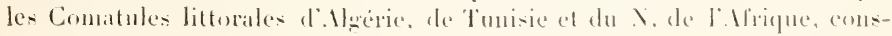




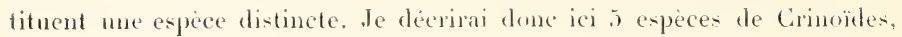
dont les caractèress sont résumés dans le tableau suivant:

\section{TABLE $\backslash U$ DES ESIECES}

1. Pl. e.dors. tres qrande ordin. fres próminente el fortement conique, le long de laquelle les cirres sont dispones en rangées vertic. ; res cirres sont trè- lomgs et complemment 35 a 601 art. : les 3 premieres pl. loracls. sont distimetes. Conlent verte a l'état

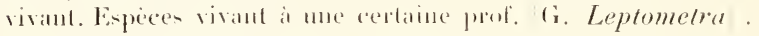

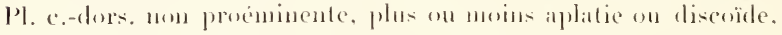

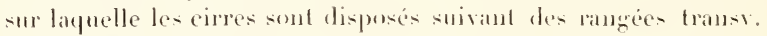
Ces cirres sont assez courts el lemrs ardicles sout peu mombrems. me vinglaine an plus : les deux premieres pl. brach. seules sout visibles extér. Coulenr rouge on orangée sm le virant : esp. surtout litlorates. (r. Antedon

$\because$ Cirres très longs ; tous les art. sont notablement plus lougs que larges ; les bras ont $150 \mathrm{~mm}$, de long. en moyenne et les cirres 50 à $6010 \mathrm{~mm}$. . . . . . . Leplometra phalangium p. 195

- Cirres relativement plus conrts; les art. proxim. sont environ 2 fois plus longs que larges, mais les art. dist. sont presque ausis larges que longs, et tout au plus $1: 3$ plus longs que larges. Les luas ont environ $125 \mathrm{~mm}$. de long. el les cirres 35 à $50 \mathrm{~mm}$. .

L. cellica p. Is

2. Chaque cirre comprend 18 it 20 art. Lous allomeres, les art dist. it peine dillérents des prox. el non comprimés later. Couleur urangée. . . . . . . . Antedon mediterranea p. 195

- Chaque cirre comprend au plus 17 ant. ef souvent 15 seulement : les art. prox. sont plus longs que les dist. qui sont eomprimés latéralement

4. Les art. dist des cirres ne ditlérent pas beanconp des prox. La longe. des art distaux plus courts, mesures sur lem bord dors., est d'un tiers ou dime moilié sup. à lemr diam. lat. Coulemrouge.

- Les derniers art des corres sont lortement comprimés latér. el lorsquion les regarde de probil lemr reg. dist. est e fors plus grande

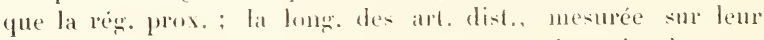
bord dors., est égale a lenr large sur leur bord prox.

- A. maroccana. (p. 19\%.) 


\section{F. ANTEDONIDÆ Nomun.}

Crinoides libres a l'etat adulte: pl. c.-tors. portant un certain nombre de curres; bras an nombre de fo. Les pl. bas, sont invisibles exter. et forment une rosette incluse dans le disque. Bouche centrale el anus excentrique.

\section{G. ANTEDON Fínistille.}

La pl, c.-dor's., arrondie ou discoïdale, n'est ni conique, ni très proéminente, et les cirres quielle porte sont disposés suivant des rangées transv. Ces cirres sont assez courts et les art. sont au uombre d'une vingtaine au plus. La première pinnule est allongée, ? fois plus longue que les autres, formée d'art. fins et allongrés : la $2^{e}$ pinnule ressemble aux suirantes. Les parois du calice sont constituées par 2 radiales primibrachiales et par les 3 premières brach. secondibrachiales. Formes liltorales ; couleur rouge, rouge orangé ou orangé.

Je décrirai surtout lesp. commune sur notre littoral de lit Méditerranée. dont les 2 autres ne diffèrent que par des caractères plutò second.

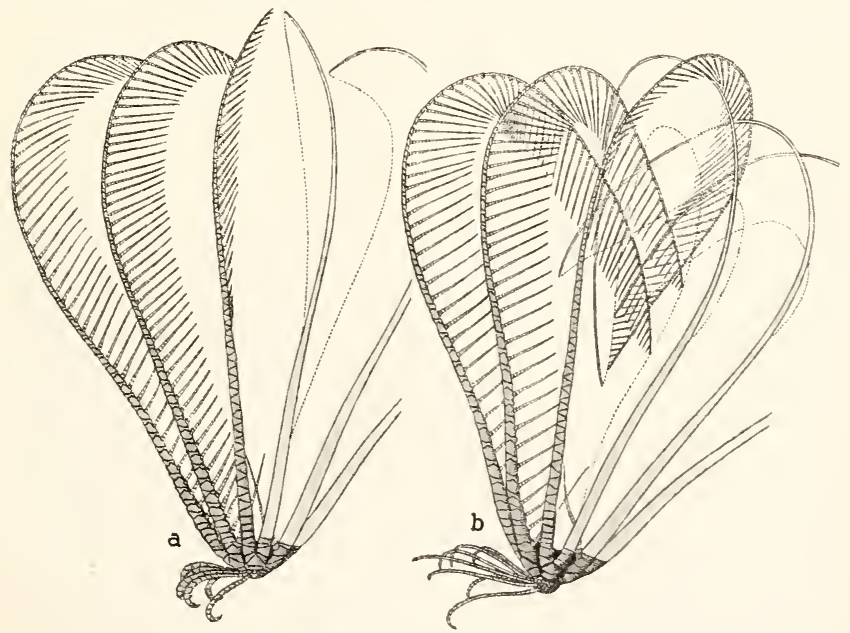

Fin. 149. - a. Antedon bifida; b. A. mediterranea; fig. schimatiques d'aprés A. Clink.

A. mediterranea Lamarck. Fign.:149 b et 150. - Voir : 1. Chakk, 1915 , p. 169, fig. 105, et 191ヶ, p. 203.

Le calice a la forme d'un còne très surbaissé dont le sommet arrondi est 
recouvert par la pl. c.-dors.; ses parois sont surtout fomées par les 2 primibrachiales, la première n'étant pas visible, el le reste par les 3 premières secondibrachiales: entre ces art. les espaces étroil- qui restent libres sont simplement membraneux comme l'est aussi la base du cone qui représente la lace orate: les 5 bras sont donc constitués chacun par 2 art. seulement, la hifurcation s'ellectuant après le 2" art. Les syogres se trouvent entre les lirach. 3 et 4 , puis elles se montrent assez régulièr. entre les brach. 9 et 10. 1 í el 15. Is et 19, 22 el 2:3, etc. La première pinnule de chaque bras est beatucoup plus longue que les autres et ses art. successifs sont allongés: elle renl'erme 35 a ís art. : la long. des pimule-suivantes dépasse nn peu la moitié de la premiere. Je diam. dueatice est de ti a 7 mm.. les bras alteignent une long. de $100 \mathrm{n}$. en movemue. Ja pl. c.-dors.. -implement eonvere ou meme quelque pen aplatie, porte 25 it in cirres dont la lomgre moyenne ent de 1.5 it $16 \mathrm{~mm}$. : chacum deux est constitué par lis a 20 art., lous allongés, exeepté les :2 ou 3 premiers: les art. dist. diffierent a preine des pros. et ils ne sont pas comprimés latér. Ces cirres soml disposés en rangés lranss. plu- ou moin apparentes et le milieu de la ce-ders. reste libre.

La coulemr a l'elat vivant est orangé on janne orangé.

L'A. mediterranea est répandue sur loutes nos coles méridionales, de Banyuls it Menton, à une faible prof. parmi les Mlgues, contre Ies rochers ou fixie it laide de ses cirres sur dirors animitux. Elle est romue sur les coles d'Espranc, au X.

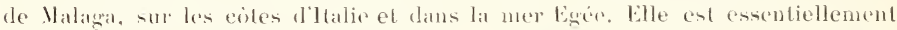
littorale. Lin mine femps que les adulles, on rencontre sourent des jeunes ì tous les élints de divalopjentent.

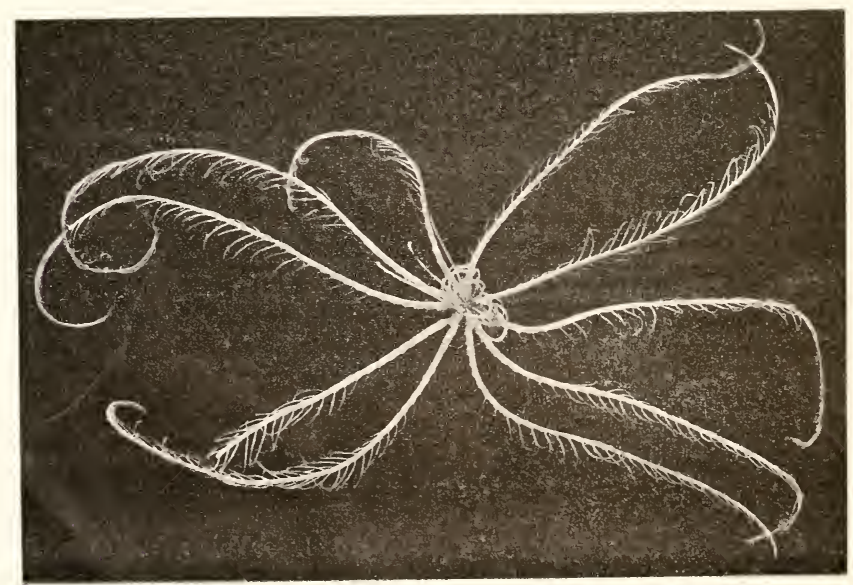

1.16. 150. - Antedon meditereanea, stumelenr naturelle, 


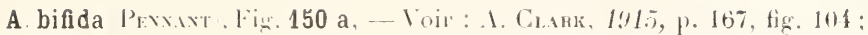

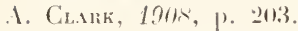

L'.1. bifida ollre la mème structure que l'.1. medilerranea; elle en diffère seulement par ses bras plus courts, leur long. movenne ne dépassant général. pas 70 i so $111 m$. e par ses cirres beaucoup plus courts égal., ceux-cin'ayant pas plus de 17 art. et souvent 15 seulement : ces art. sont plus courts, mais relativ. plus larges que chez l'd. medilerranea, et les art. prox. sont plus longs que les art. dist.; la long. de ces cirres est de $12 \mathrm{~mm}$. en movenne.

La coulem de l'A. bifida est rouge, rouge pourpre, rosé ou rouge orangé.

L'A. bifida est très répandue sur nos eóles de la Manche et de l'Atlantique et peut ître capturée en nombreux exempl. lors des prandes marées ; elle est ordin. fixée par ses cirres aux pieds des \%ostères ou des Ilgues, parfois aussi sur les rochers. Li. bifida patrait manpuer daus le Pas de Calais mais elle se retrouve sur les colles dingleterre.

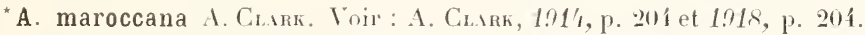

Cette forme, qui vit sur nos eôtes d'.lgérie et de Tunisie est extrêmement voisine de J'A. bifida el mérite à peine d'en ctre distinguée spéeifiquement; on pourrait n'en faire: qu'une var. de l'A. bifirdn différ'int du type par les derniers art. des eires fortement comprimós litir.

\section{(i. LEPTOMETRA 1 . Ci.AнK.}

La e.rlors. très déreloprée est fortement prosminente, conique, aree le sommet tantit arrondi, tantit assez pointu. Les eirres sont liposés en rangées vertic. plus ou moins régul. : ils sont très long's et les art. dist. n'offrent pas de crête dors. mais restent arrondis. Les parois du ealice sont formées par 3 rad. suecessives (primibrachiales el par les f premiers art. brach. (secondibrachiales). Les 2 premières pinnules de ehaque bras sont beancoup plus longues que les suivantes. Les 2 especes du g. sont assez rolustes, leurs bras sont très longs ainsi que les cirres; la long. des bras varie entre $: 00$ ot $150 \mathrm{~mm}$., et celle des cirres entre $3 \ddot{3}$ et $160 \mathrm{~mm}$. Ces 2 espèces u'abandoment jamais une certaine prof. / de 50 ¿t $1.2 \times 0 \mathrm{~m}$.). Les animaux sont d'un bean vert à l'état vivant.

L. phalangium. O. F. Mülefer. Fig. 151, 152, 153 a. - Voir : Mirmov, 1879, p. 40. pl. XVIII Anledon p. ; Cinpextfr, 185.5, p. 17.5, pl. IIII: 1. Ciark, 1918 , p. 231.

Celle espèce, exclusivement méditerranéenne est plus grande el plus robuste que l'Antedon mediterranea; les bras sont plus longs, et surtout les cirres offrent une très grande long.; leurs arl. sont grêles et allongés et ils suinèrent sur une c.-dors, conique, ordin. 2 fois plus longue que large $152 \mathrm{a}$, pourant afteindre $5 \mathrm{~mm}$. de long.. quelquefois cette c.-dors. est plus courte (b), mais elle reste toujours très saillante. Les cirres sont au nombre de 25 à 30 , et leur long., est variable : les plus petits ont $25 \mathrm{~mm}$. de long., 
mais les plus grands atteignent 50 a $60 \mathrm{~mm}$. ; on compte 37 a 38 art. dans les plus petits et une cinquantaine dans lesplus grands. Les premiers art. sont plus larges que longs, puis la proportion change, les art. s'allongent et ils finissent par être 2 et même 3 lois plus longs que larges 153 a). Tous sont com-

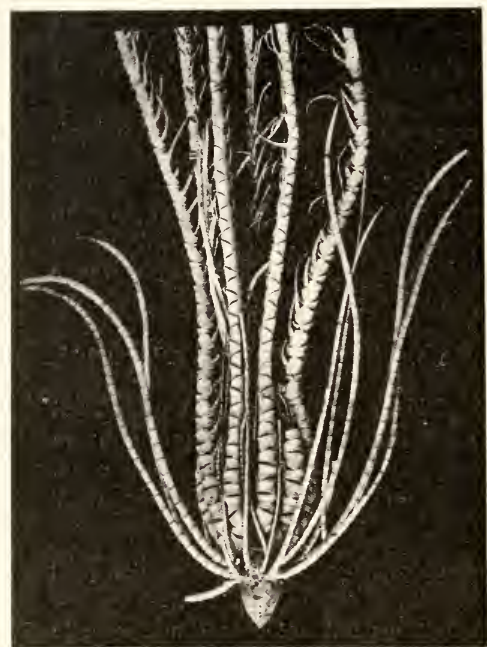

Fir. 151. - Leptometra phatangium: vere laterale, $X: 2$.

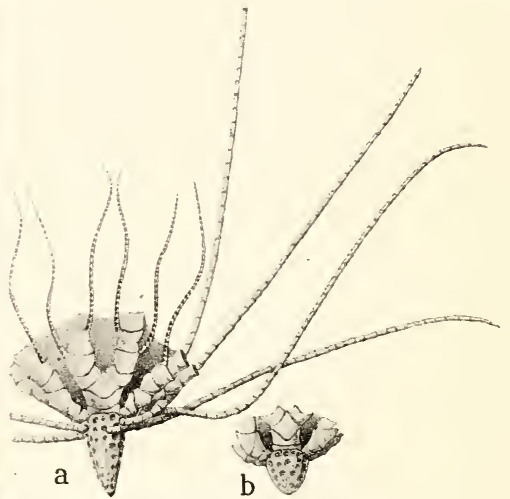

Fig. 152. - Leptometra phalangium; caliec (dapres Maniox) ; a, plaque centro-dorsale très allongée; $\mathbf{b}$, plaque centro-dorsale raccoureie; $\times 3$.

primés latér. : le dernier art. forme un crochet allongé. les bras mesurent 120 a $150 \mathrm{~mm}$. de long. ef présentent 200 art. en movenne. La première -rygie se trouve entre les art. 3 et $\{$, lat suivante ordin. entre les art. 10 et 11 : puis les syzygies se suivent i les intervalles variables, mais rapprochés (tous les 3 ou 4 art. général.).

1:A. phalangium se troure fréfuemment au large de nos côtes de Provenee, dams les fonds vaseux, it partir de 70 it $80 \mathrm{~m}$. : elle devient plus abondante entre to0 of 20010 ., dans les graviers of les fonds coralligènes.

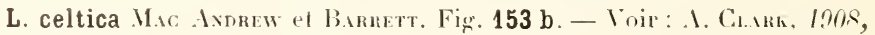
p. 231.

La Leptometra celtica, essentiellement atlantique, diffère de la forme méciiterranéenne par quelques caractères, pen marqués à la vérité, mais qui ont paru suffisants ì certains auteurs pour justifier une séparation spécifique. 


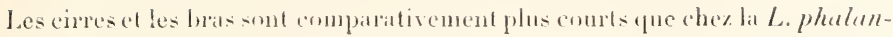
gium; les cirres, an nombre d'une trentaine ont 35) it il mum. de long. tout au plus: ils comprennent it peu press le mène nombre d'art. que chez la L. phalangium, mais ces arl, se raceourcissent beaucoup dans la rég. des

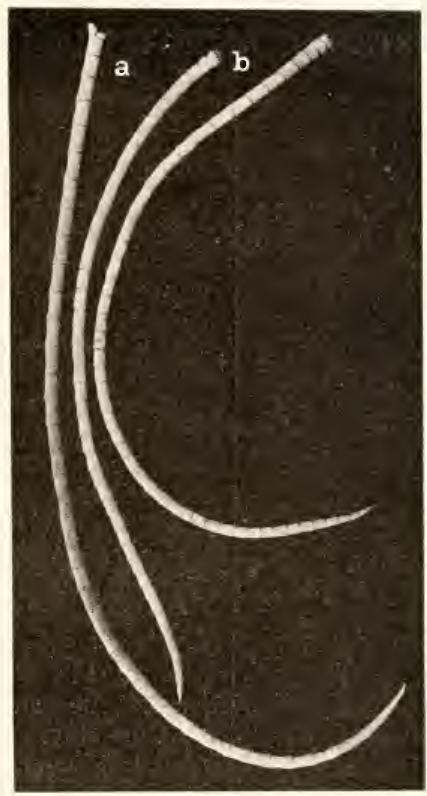

Fur. 153. - Cirres te Leptometra; a, L. phalangium; b, L. cellira (d'aprés II. Cahpextek).

cirres ef arrivent ì ètre à pen pris anssi longs que larges, on à peine un pen plus longs que larges.

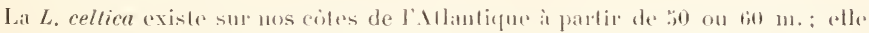

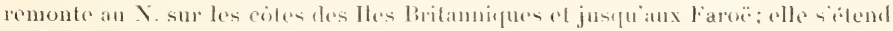

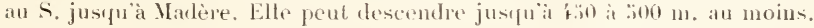




\section{I.ISTE DES IBREVIITIONS}

adambul., adambulacraire.

alternat., alternativement.

ambul., ambulacre, ambulacraire.

art., article.

auct., auctormm.

bas., hasal.

brach., Jirachisl.

r. it d., cest it dire.

calc, calcaire.

curin., carinal.

c.dors., rentro-dorsal.

centr., ecutral.

Cl., classe.

$\mathrm{cm}$., centimètre.

complet., conplètenent.

corb., corbeille.

corpusc, corjuscule.

dent., dentaire.

diam., diametre.

diq., digestif.

dim., dimension.

dist., distal.

dm., llécimètre.

dors, dorsial.

dorsal., dor'salement.

E., Est.

echunt., śchantillon.

egal., également.

esp., espèce.

exart, exactement.

exceptionn., exceptiommellement.

e.rempl., exemplaire.

e.rt., cxterue, extérieur.

p.rter., trírieurement.

p.rtrim.. extrimentent.

$r, f$, fimille.

fuse fasciole.

F., g., genre. gènèral., généralement.

genit., génital.

gl., glande.

glandul., glandulaire.

globif., globifère.

labil., habituellement.

haut., hauteur.

ident., identique.

indiv., individu.

int., interne, intérieur.

interr, intérieurement.

interrad., interradius, interradial.

imrigul, irrégulier.

irrégnlièr., irrégulièrement.

l., Limné.

larg., largeur.

lat., latcral.

latèr., latéralement.

latit., latitude.

lègèr., légèrement.

local, , localité.

long., longueur.

longit., longitude, longitulinal.

longituel., longitudinalement.

m., milre.

madrép., madréporique.

marg., marginal.

$N$., Nord.

$O$., ordre.

obl., oblitjue.

obliq., obliquement.

ophir. opluicépbale.

ordin., ordinairement.

ory., ormane.

orif., orifice.

particul., particulièrement.

pax., paxille.

perdir., pédicellaire. 
jerfor., perforation.

periph., périphéric.

périphèr., périphérique.

péripr., périprocte.

perist., péristome.

péristom., péristomien.

perpendic., perpendiculairment.

piq, piquant.

p., page.

pl., plaque (planche dans les reuvois bibliographiques).

$p v^{*}$, dit, proprement dil.

prim., primaire.

princip, principalement.

prof., profondeur.

prox., proximal.

$R$, longueur du radius.

$r$, longueur de liuterradius.

rad., radius, ratial.

règ., région.

régul., régulier.

régulier., régulièremont.

relal., relativement.

s., sud.

s., , ., sous-classe. srlèr., sclérile,

second., secondairr.

S. (1., sous-ordire.

squel., squelette.

sup., supérieur.

supere, supéricuremrnt.

superf., superticiel.

surf., surface.

tég., tégument.

tentac., tentacule, fontaculaire.

term., terminal.

transi., transwersal.

trunsters, transversalement.

tridact., tridactyle.

trif., trifolié.

tuberc., tubercule.

var., variété.

rent., rentouse.

rentr., ventral.

rentral., ventualement.

rert. vertical.

vertic, verticalement.

rèsic., résicule.

W., Ouest. 


\section{INDEA BIBLIOGRAPHIQUE}

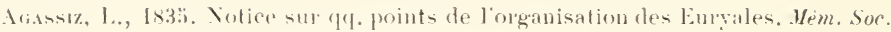
Sc. leuchitel. T 11.

Agassz, A., 1872-1874. levision of the Echini. Illustr. Catal. Mus. Comp. Zool. Cambridge. $\mathrm{N}^{\circ} \mathrm{\tau}$.

Agassiz, A. el Crark, 1., I908. Jawain and olher Pacific Echini. Mem. Mus. Comp. Zool. Cambridge. Vol. XXXIY (la suile et la fin par L. Clark, 1912-1917).

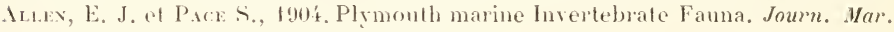
Biol. Assoc Vol. III, No 2. Plymouth.

B.mmoıs, Thı, 18x2. Catal. des Crustacés Podophthalmaires et Échinodermes recueillis à Concitrneau. Lille.

Ber.t, J., 1891. Asterius mbens and the British species allied thereto. Ann. Mag. Nat. Hist. 6, Vol. VII. London.

Bell, J., 1892. Calal, of the British Echinoderms in the British Museum. London.

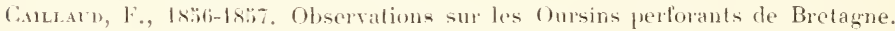
Rex. Mag. Zool. (2), No 8, p. lis, et No 9, 1.1.

C.mpenter, II., 188:. On the variation in the form of the Cirri in certain Comatulit. Trans. Linn. Soc. Londor. Sér. 2. Zool. Vol. IJ, p. tï.

Crakk, Instin, l!9t'. Buituäge zur liemntnis der Vecresfamen Westafrikas. Echinoderma II. Crinoidea. Ilambourg.

Crank, Austin, 191:i. I nomograph of the existing Crinoisls. Vol. I. The Comatulids. Smilh. Listit. Bull. 82. Washington.

Clark, Instin, 1918. The mnstalled Crinoids of the Siloga Expedtion. Siboga Exp. Monogr. XIII. Leyden.

Clark, Lyman, 1907. The aportons 1Folothurians. Smiths. Contrib. to knorledge. Vol. XXXY. Washingtom.

Crark, Lrman, 1913. Calat, of the recent Ophiurans. Mem. Mus. Comp. Zool. Cambridge. Vol. XXV, No f.

Cravor, l.., Éndes morphologiques sur les Échinodermes. Arch de Biologie. Vol. $\mathrm{XI}, \mathrm{l}, 313$.

Crivor, L., 1912. Fanne du hassin d'Jreachon. V. Échinodermes. Bull. St. biol. Areachon, l'te annive, lase. I.

Demafe, Y. et llínotari, E. 1903. Traité de Zoologie conerète. T. IIl. Les Échinodermes. Paris.

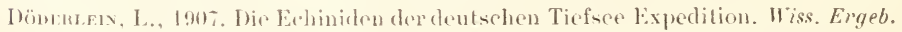
1. Tiefsee E.r.p. Vol. V.

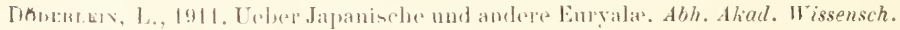
München, suppl. Bit. II. Munich.

IH wax, P. Il., el St.mix, P., Issl. Eehinodermata of the Arelic sea to the West of firendanel. Jomiton.

Fonses, E., 18i:?. () the Radiata of Eastern Mediterranean. Trans. Linn. Soc. l.onken. Vol, NIX, p. li3, 


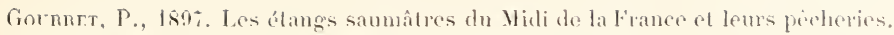
Ann. Mus. hisı, naturelle. Marseille. Vol. V, Mém, no 1.

IJezen, C.. 1863. Wnters. üb, die Lilloralfauna des adriatischen Meeres. Silz. Mat. Nat. Aliat. Wiss. Nien. Vol. XINT, p. 113.

Ifrerer, C., 1868. Die Zoophyten und Echinodermen des adrialischen Weeres. Wien.

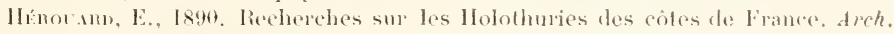
Zool. E.rp. 2, Vol. VII. Paris.

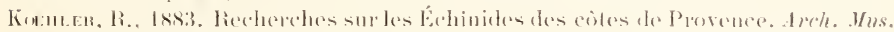
Mist. Vat. Marseille. Vol. 1, X"3.

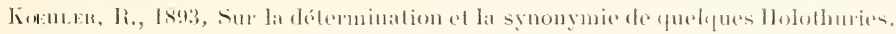
Bull. Se. France el Belgique. T. XXV. Paris.

Kinuten, R., 189. Echinodermes recueillis a La Ciotal. Mem. Soc. Zool. France. T. VIl, I,ille.

Kinuler, R., Ix9:3. Yotes échinologriques. Rerue biol. vord de la Franre. T. VII. Lille.

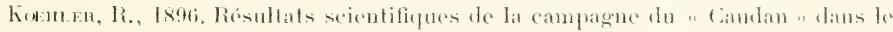
golfe de Gascengue. Échinodermes. Ann. Lnix. Lyon, Vul. XXV1.

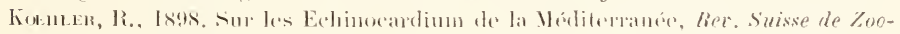
logie, T. VI. Gienive.

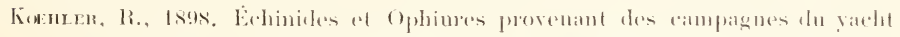
"Hllirondelle ". Ries. campagnes scientif. accomplies par le Prince de Monaro. Fase. NII. Yonateo.

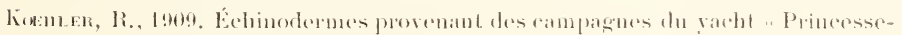

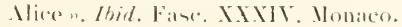

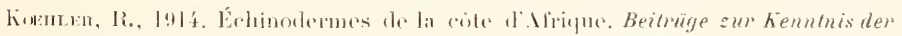
Meeresfaumen Hestafrikas. Echinorlermata 1. 11ambourde.

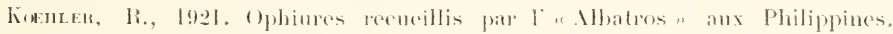
IVashington (sous presse).

Konmlsh, R., 1921 a. Astéries recueillies par l' "Expédition antarelique Australiemne ". Srolner (sous presse).

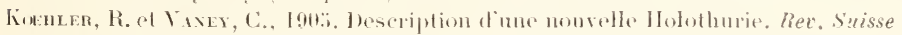
de Zoologie. T. XIII, fase. I, 1898.

I.uxg, A., Traité danalomie compraréc el de Zoologie. T. Il. Mollusques al Éehinodermes. Trad. liançise. Paris.

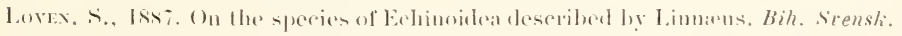
let. Aket. Hendl. BH. NIII.

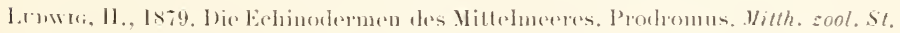
Neapel. Bul. I.

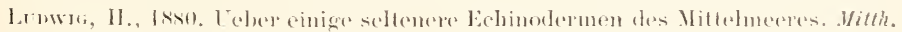
Znol. sit. leapel. Bul. II.

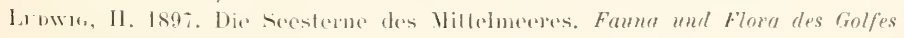
von Veapel. Monogr. XXIV. Bertin.

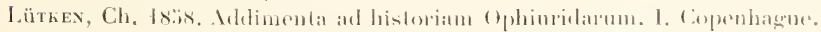

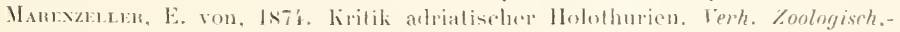
botunischen Gesellsch. Wien. Bd. XXIV.

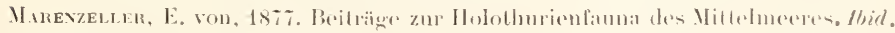
Bd. XXTH, p. IIR.

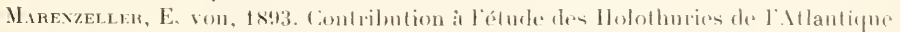
Nord. Res. Camp. Scientif. Prince de Monaco. l'ascicule Xl. Mrnaco, 
Varmos, A. F., 1679. Dragages an large de Marseille, Inn. Si. Nat. Zool. of. Vol. VIII.

Уatsunoto, II , 1917, A Monograph of Jipanese Ophiuroiclea aranged aceording to a new Classification. Journ. of the College of Science Imp. Univ. Tolio. Vol. XXXVIII, art. 2.

M.sssy, 1., 1920. The IIolothumoidea of the Coasts of Hand. Scient. Proc. Roy. Soc, Dublin. Vol. XVI, No i, p. 3i,

Montexisex, Th., 1903. Echinoidea I. The Danish "Ingolf" Expedition. Vol. IV. Copenhague.

Mortmasex, Th., 1907. Eehinoidea II. Ibid.

Mortensiz, Th, 1913. Die Echinodermen des Mittehmeres. Mtth. Zool. St. Neapel. Bd. NXI, No 1 .

Mälren, J. et Troscmer, F. II., 1X'2. Sistem der Asteriden. Braunschweig.

Ontox, J. II., 191't. (H) some Plymouth IJolothurians. Journ. Mar. Biol. Assoc. Vol, $\mathrm{X}, \mathrm{n}^{\circ} 2$.

Ostengrex, II., 1898. Zur Inatomie der Dendrochiroten nebst Beschr, nener Arten. Zool. Anz. Bd. XXI.

Ostergnes, II., I90\%. Zur Kenntnis der skandinavischen und arktischen Synaptiden. Arch. Zool. Exp. ('́). Vol. III

Ostergin: II., 1906. Einige Bemerkungen über die Westeuropäischen Pseudocucumis - und Phyllophorus - Arten. Arkiv for Zoologi, Bd. III, No I8.

P.ce, S., 190't. Notes on two species of Cucumaria from Plymouth. Journ. Mar. Biol. Assoc. Vol. VII, p. 30 :.

Penner, Erl., 1879. Róvision de la collection des Stellérides du Musemm. Arch. Zool. Exp. Vol. IV et V. Paris.

Promer, Ed., 18xt. Contribution à I'étude des Stellérides de l'Allantique Nord. Res. Camp. scientif. Irrince de Monaco. Fase. XI. Monaco.

Pfrnier, R., 1902. IIolothuries. Exp. scient. "Travailleur " et "Talisman ", Vol. V, Paris.

Protuo, II., 1888. Recherches sur Ie Dorocidaris papillata el quelques autres Échinides de la Méditerranée. Arch. Zool. Exp. (2), Vol. V.

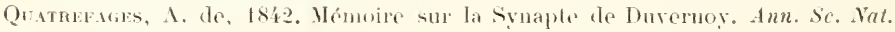
Zool. (2), 1. VII, P 19 .

Rr sso, A., 1893. Speeses di Eehinoderni poen conoscinti e movi riventi nel Golfo di Neapoli. Mem. acad. Neapoti. 1893.

Sins, M., 18.is Bidrag til Kundskaben om Viddelhavels Littoral fauna. Myt. Magaz. f. Vature. Pd. IX, Christiania.

Sitexk, E., 18tit. Beiträge zur Inatomie mol systematik der IIololhurien. Zeil. f. wiss. Zool. BH. XVIII.

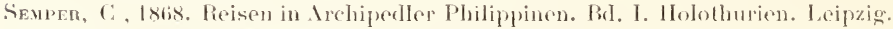

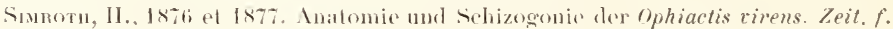
wiss. Zool. Bi. XVII el XYIII.

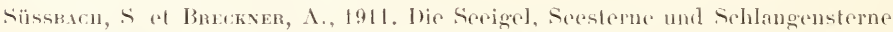
del Nord - und Ostsee, Labor. f. intern. Meeresforschnng in Kiel. No 17.

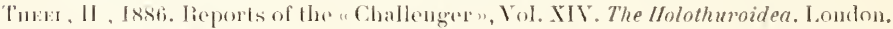
Vemul, 1., 191' . Monograph of the Shallow-water Starfishes of the North Pacifie Coast from the Aretic Ocean to the California. Smillsonian Institution, Harriman Alaska series. Vol. XIV. Washington. 


\section{INDEX SYSTEMATIQUE}

Cet index comprend tous les noms employés dius la partie systémalique. Jes classes sont en capitales grasses, les sous-clesses en capilithes grasses inclinees, les ordres en capilales ordinatres, les sons-ordres en capitales inclinces, les familles an minuscules grasses, les genres feommencant par me majuscule), les especes cl les carietes (commencant par mo minuscule), en romaines pour les noms corrects, en ilaliques pour les synonymes (1). Chaque nom est suivi du numéro te la page correspondante en chiffres ordinaires, el, sil y a lieu, de celui de la figure en chillies grats. Les numéros eutre crochels apres un non de genre sont ceux des pages oi il at cili simplement en symonymie. Les lormes non enerere signalées arec certilude sur les coles de france sensu stricto sont précédées d'un astérisque.

abildyaardi (Ophiothrix fragilis), i9,

76 a.

aculus lichimus), $116,77,78$.

iecuiluberculata (Al)acia), $113,75$.

affinis (Cidaris), 110.

affris (Hololhuria), Ino.

affinis (stylocilaris), 110, 72, 73.

athida (Ophura), 92, 61 .

alopecurus (")phiolhrix). Ai.

Amphidelus, 13i, 136i, 13s.

Amphipholis, 8:.

Anpluiura, $88,82$.

Amphiuridæ, $7 \times$.

angulosus (Echinocyamus), 1:2.

Anhyroderma, Ixi.

- amuulosa (Ophiopsila), 96, 64.

Inseroporla, 33.

Interlon, 19\%.

Antedonidæ, 19:.

APODES (HOLOTHURIES), L'i. aranea (Ophiopsila), 9\%, 63.

Irbacia. 11:3.
Arbacina, $11:$.

arborescens (Astrospartus), 6.;, 43.

"arguinensis (Itolothuria), 17i.

Asmuocmimotes, 170.

Isterials, 23, 22, 26 .

Asteriidæ, 21.

Asterina, 32.

Asterinidæ, 3\%.

Asteriscus, 32 .

Asteropidæ, '?.

Istripecten, $t i$.

Astropectinidæ, i $i$.

Astrophyton, 6it.

Istrospartus, 6.:.

allenuala (IIacelia), in, 29.

aurantiaca (Thyone), 167.

iurauliacus (Istropecteu), it, 32

balli (cpluinelis), 83, 55.

bitirlit Intedon), 19\%, 149 a.

bispinosis (1stropecten), iti, 33, 34.

botellus Holothuria), 173.

brachiata (Ophiocnida), sti.

(1) Dans la synonymie donnèe pour chaque espece, les noms specificues inlentiques au nom précédemment indiqué ont èti, pour gagner le la place, réduits a l'initiale, comme les noms génériques tans les mémes conditions. Un les tromvera ici in extenso at accortes aver charue nom de genre comme ils doivent bien entendu lêtre. 
brachiatun (()phiocentrus), xtj, 57.

Brissopsis. 131.

lirissus, 133.

Inumea (Cucmuaria, 16i, 117

(analiferus (Sehizaster), $128,88$.

(atanensis (Holothuria), 179.

celtica (1eptometra), 178, $163 \mathrm{~b}$.

Centrechinidæ. 111.

Cintrostephanus, 11:.

Ciermaster, 4?.

Chataster, 30.

Chætasteridæ, :36.

chiajei (Imphiua), $78,50$.

CHILOPHIURIDES, ๙ 7 .

Cidaridæ. 10\%.

Cillaris, 109, 110.

ciduris (Culluris), 10!?.

ciliatris (Luillia), :3: 41 a.

ciliaris ophiura, 90.

Cesprastumes, 126.

columbaris (Brissus), 13.3.

Comatula, 193.

corlatus tmphidetus), 13't.

corrlatum Echinocardium), 13t, 93.

Coscinasterias, 2.).

ciribrella, 30, 31.

CRINOIDES, 191 .

Crosstzster, 3.i.

Cineumaria, l:30, [169

cucumis (Cucumaria) 1ti1, 114, 115.

cuenoti (pseudocucumis), 168.

DENHROCHnotes, (::0).

dicquemarii (Cucumaria), 1:í.

digitata (aabidoplax), 188, 144.

lligitalı (synapta), Ix8.

di-Stefanoi (-spatangus purpureus,

131.

doliolum ('incumaria), 1:33.

borocidaris, tos.

duvernea (synata), 187.

Echinaster, 2!.

Echinasteridæ, :?!.

echinutu (Ophiothrix. i:.

echinata (Ophiothix fragilis), 7ï, 48.

ÉCHINIDES, $9 \%$.

Echinocarlium, 13i.

Echinocyantus, 1:66.

Echimus, 116, 1:21, 1:2.2. edmundi Asterias), 20.

elegans Amphiura), 8:2.

elegans (Thyone), 1tii.

clongata (Cucumaria), 160, 112, 113.

asculentus (Echinus), 119, 80.

Euryale, biti.

farcimen (Holothurio), 171.

filiformis (Amphima), $\times 1,52$.

tlavescens (Echincardimm), 136, 95,99.

forbesi (Ophioconis), 8!), 59.

l'ORGaPt LOSÉES, 21.

Porsliali (Ilololluuria), 179, 135.

laagilis (Ophiothrix), it, 48, 49.

fusus (Thyone), 16i, 118.

gradeanu (Thyone), $16 \%$.

galliemei (Leptosmapta), 1×6, 141.

Genocidaris, Il't.

yibbosa (Asterina), 3:2, 24.

grbbosus (Amphidetus), 138.

glacialis (Asterias), 22.2.

glacialis (Marthasteriats), 2.2, 17.

GNATHOPHIURIDES, io.

Gorgonocephalidæ, 6:i.

Gorgonocephalus, 6i;.

granularis (Spharechinus), 12', 85, 86.

"grubei (Cucmuaria), lï, 105.

guernei (Sclerasterias), 27, 20.

Hacelia, 39.

Haplodactyla, 18x́.

helleri (1tolothuria, 180. 136.

Hemricia, 30.

Ilolothuria, 171.

HOLOTHURIDES, $1: 0$.

Holothuriidæ, $17 \%$.

"Hrdrasterias, sti.

hynduani (Cucumaria), 15\%, 109.

impatiens (Ilolothuria), 173, 129.

inermis (Thyone), 16\%, 123.

inherens Leptosynapta), 187, 142.

intermedium (Echinocardium), 137.

irregularis I-tropecten , 50, 37, 38, 39 .

I RRÉGULIERS (ÉGHINIDES),

$1: 26$.

jonstoni Istroprecten, 4!!, 36.

kirschbergi (Cuenuaria, 1:̈t, 108.

köllikeri (tucumariat, 196, 107.

Labictoplax, Isx.

laceretosal ()phimar), 90, 60. 
lactea (incumaria), 163, 116.

\section{LAMOPHIURIDES, 69.}

leferrei (Cucumaria), 152, 102.

*lentiginosa (Holohuria, 17t.

Leptometra, 19\%.

I.eptosynapta, Isti.

lividus Paracentrołus, 12:3, 84.

lividus strongylocentrotus, 123.

lixula trbacia, 113.

longicauda (Ophiodernai), $\times i, 58$.

longipes (hintaster), 37, 27.

longispinus (Centrostephanus, 113,74 .

luidia, io.

\section{Luidiidæ, :i...}

lusitanica (Ophiothrix fragilis), it, $49 \mathrm{~d}$.

lütkeni Ophiothrix), il, 46.

IYrifera (Brissopsis , 132, 91.

maculata (Genocidaris, $11:$, 76.

maculatus (Temnechinus), $11 \%$.

"makrankyra Leptosynapta gralliennei, $18 \%$.

mammata (llolothuria), 175, 133.

mamillata Holothuria), $1 \%$.

marioni (Cucumaria), 169.

marioni Pseudocucumis), 169, 125.

- maroccana (Antedon ', 197.

Marthasterias, 21.

mediterranea (Amphiura), 79, 51.

mediterranea (Intedon), 195, $149 \mathrm{~b}$,

150.

mediterranea (Haplodactyla), 184.

mediferraneum Echinocardium, 13\%.

94.

melo Echinus), 118, 79.

membranacea (Anseropodia), 33, 25.

membranacers (Palmipes), 33.

microtuberculatus (Echinus), 122.

microtuberculatus (Psammechinus),

$122,81 \mathrm{~b}, 83$.

miliaris Echinus), 121 .

miliaris (Psammechinus), 1:1, 81 a, 82 .

mixta (Pseudocucumis), 168, 124.

Molpadia, 1s't.

Molpadiidæ, 18't.

montagui (Cucumaria), 1.50, 101.

mortenseni Echinocardium), 137, 96,

98. musculus (.1nkiyroderma), Isi.

unseulus (Molparlia), Ixi, 139, 140.

neglecta Amphim'a), x?.

neglecti stylasterias, 26.

nigra (Holotheria). 179.

nigra Ophioeomina , 93, 62.

normani (Curumaria), 1:i2.

ocnlata Ciribrella), 31.

Ophiacantha, 69.

Ophiacanthidæ, 69 .

Ophiactis. 83.

"ophidianus (Ophidiaster), 3×, 28.

- Ophidiaster, 3r.

0phidiasteridæ, $3 \times$.

Ophiocentrus, x.i.

Ophiocnida, 86.

ophiocoma, $9 \mathrm{i}$.

Ophiocomidæ, 93.

Ophiocomina, 93.

Ophioconis, 89.

Ophioderma, si.

Ophiodermatidæ, si.

Ophiolepididæ, 90 .

Ophiomyxa, 67.

0phiomyxidæ, $6 \pi$.

Ophiopsila, $9 \mathrm{i}$.

Ophiothrichidæ, 70.

Ophiothrix, 70.

Ophiura, 90.

\section{OPHIURIDES, 58.}

pallaryi (Arbacina), 113 .

ovalus (Amphidefus), 136.

ovatum (Echinocardium), 136.

Palmipes, 33 .

papillata (Dorocidaris), 109, 68, 70, 71 .

papposus (Crossaster), 3:.

papposus (Solaster), $33,26$.

Paracentrotus, 123.

Paramphiura, 83.

Paxillosées, $\mathbf{t}$ í.

Pectinura, 89.

PÉDIFĖRES (HOLOTHURIES). 150 .

pennatifidum (Echinocardium), 138,

97, 100.

pentacanthus (Astropecten irregula-

ris, : $1,38$.

pentagona (O)hiomyxa), 67, 44. 
Pentagonaster, iz.

pentaphrilum (Ophiothrix fragilis), 6 ,

$49 \mathrm{~b}, \mathrm{c}$.

phalangium (Leptometra), 197.

PHRYNOPHIURIDES, 6.5.

Phyllophorus, 169.

placenta (Ceramaster), \{2, 31.

placenta ('almipes, 33.

placenta Pentugonaster), $\$ 2$.

planci (Cucumania), 133, 103, 104.

platyacantlus (Astropecten bispinosus). 47.34.

Plutonaster, $\mathrm{it}$.

polii (IJolothuria), I ix, 134.

Porania, 41.

poucheti (Thyone), 1 ti.i.

PMIXY

PHYMOUE⿱

Psammechinus, 121.

Pseudocueumis, 168.

pulvilus Porania), 41.30.

punctata Parampliura), 83. 54

purpureus (Spatangus), 129.89, 90.

pusilhus (Echinocyamus), 127, 87.

pustulosa (Arbacia), 113.

quinquenaculata (Ophiothrix), 7:, 47.

raphanus (Throne), 168. 119, 120

regalis (Ntichopus, 182, 138.

RÉGULIERS (ÉGHINIDES), 10 .

lihabetomolgus, $1 ! 1$.

- richardi (Ilydrasterias). 26 i.

richarli (Stirhopus), InI.

roseovita (Thyone), 166, 121, 122.

rosea (Stichastrella), 2x, 21.

rosens (Stichaster), 2 \%.

rosula (Ophiothrix), it.

rubens (Isterias), 23, 18.

ruber (13habdomolgus), 1!1, 146.

sanchori (Ilolothmia), 171, 127, 128.

sanguimolenta (Cribrella, 31.

saltigunterula Henticia), 31, 23.

sarsi (Luirlia), : :6, 41.

saxicole (cucumarin), 1.:0).

schizatster, 12x.

scille (Brissus), 1333.

Selerasterias, 26.

selentive (Strchopus), 179. spositus (Kchinaster), 29, 22.

serratus (Astropecten), $51,39$.

setosa (Ophiacantha), 69, 45.

Solaster, 3:.

Solasteridæ, 3. .

Spatixgmes, 128.

Spatangus, 129.

sphiera (Echinus), 119.

Sphrechinus, 12'.

SPINulosées, 29.

spinulosus (Istropecten), 48, 35.

stpumata (Anphipholis), 82, 53.

squamala (Amphiura), 82.

squamatus (Istropecten), 49.

stcllati (Holothuria), $176,131$.

STELLERIDES, 1.3.

Stichaster, 28.

Stichasteridæ, 2 i.

stichastrella, 28.

Stichopus. Ist 17!? .

strongylocentrotus, 1:3.

Strlasterias, 26.

Sirlocidaris, 110.

subinermis (Plutonaster), iit.

sulnuermis Tethyaster), : í, 40.

Synapte, 186, 18\%.

Synaptidæ, 186.

"syricusana (Cucumaria), 1.\%, 106.

Temnechinus, $11:$ :.

tenuispina (Asterias), 20 .

tenuispina (Coscinasterias), 26.19.

tergestina (Cucmmaria), liss, 110, 111.

Tethyaster, :33.

texturata (ophiura), : $1 \%$.

thomsoni (Labicloplax), I90, 145.

Thyone, 16it.

Thyonillium, 169.

tremula (Holothuria), |x|.

tremulus (stichopus), Ist.

tubulosi (IIolothuria), 17t. 130.

unicolor (Brissus), 133, 92.

urna (Phyllophorus), 169, 126.

Vintugsis, 3 r.

rerruculatus (Asteriscus), 32.

rersicolor (Ophiothrix), it.

"virens (Ophiactis), 8i, 56. 


\section{TABLE DES MATIERES}

IXTRODLCTION morphologie. embrvologic, éthologie el faunistique.

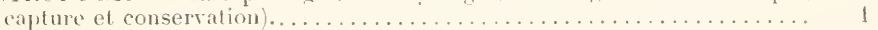

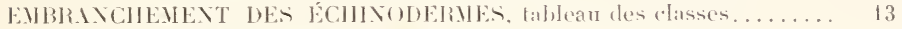

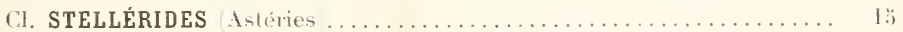

Tableau des espèces................. Ix

(). Foncıptosées. F. Asteriidæ G. Marthasterias, Asterias, Coscinasterias.

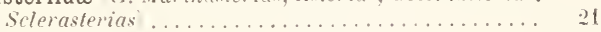

F. Stichasteridæ (i. Stichastrella ........... 2i

(). SPRtrosées. F. Echinasteridæ (i. Echinaster, Henricin .......... 29

F. Asterinidæ (i. Asterina. Anseroposla ......... 32

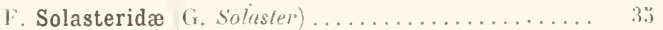

F. Chætasteridæ (F. Chictuster .............. 36

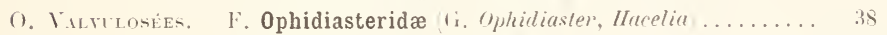

F. Asteropidæ G. Porania ................ in

F. Goniasteridæ (i. Ceramaster ............. i2

O. Puxiliosées. F. Astropectinidæ (i. Astropecten, Tethyaster....... it

F. Luidiidæ G. Luiliu .......................

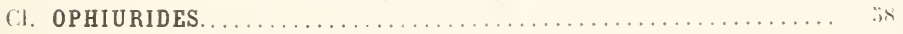

Tableau des espèces....................... 1 (il

S. CI. PHRYNOPHIURIDES. F. Gorgonocephalidæ (i. Astrosprar/us . . 6;

F. Ophiomyxidæ G. Ophiomyxa ...... 6 ir $^{2}$

.. (I. LAEMOPHIURIDES. F. Ophiacanthidæ f. Ophiucanthet .... b b!

$\therefore$ (I. GNATHOPHIURIDES. F. Ophiothrichidæ (i. ")phioth $\cdot$ ir . ... in

F. Amphiuridæ (i. Amphinra, Amphipholis, Paramphin're, Ophiaclis, ophio-

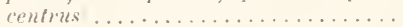

‥ (I. CHILOPHIURIDES. F. Ophiodermatidæ (i. Ophiodermo, (i)hioconis ................... si

F. Ophiolepididæ G. ()phima ........ (n)

F. Ophiocomidæ (i. Ophioromine, "), hio-

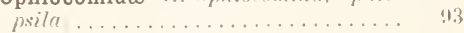

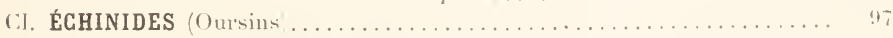

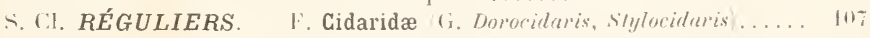

J. Centrechinidæ G. Centrostephanus, Arbacia, Genocilaris. Echinus, Psammechinus, Paracentrotus, Spherechinus ............... 


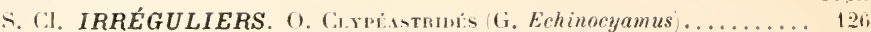

O. Sipataxgints S. O. PRYMNAUETEs G. Schizaster $r^{2} \ldots \ldots \ldots \ldots \ldots \ldots \ldots$

S. O. PRYuNodesmens G.Spatangus, Brissopsis, Brissus, Erhinocardium ...... 129

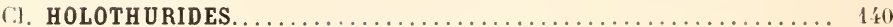

Tableau des espèces. . . . . . . . . . . . . . . . 1 '13

․ Cl. PÉDIFÈRES. (). Dexmucunnotes (i. Cucumaria, Thyone, Pseudorucumis, Phyllophorus ............. 1:0

(). Ispmocinnotes F. Holothuriidæ G. Holothuria,

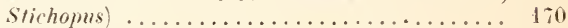

.. Cl. APODES. F. Molpadiidæ G. Molpadia ............... 184

F. Synaptidæ (i. Leptosynapta, Labidoplax, Rhabdo-

CI. CRINOÏDES molyws . ................... 186

Tableau des espèces....................... 19t

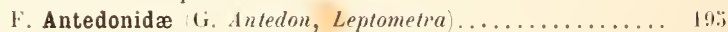

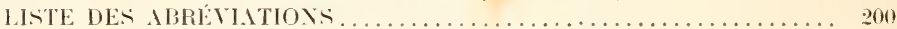

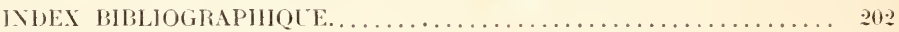

INUEX SYSTEMATIQUE........................... 20. 
FEDERATION FRANÇAISE DES SOCIETES OE SCIENCES NATURELLES

\section{OFFICE CENTRAL DE FAUNISTIQUE}

\section{FAUNE}

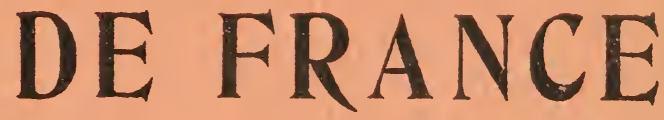

1

\section{ÉCHINODERMES}

I'AN

\section{R. KOEHLER}

PHOFESSELR A I.A FACLLTÉ DES SCIENCES DE LYON

Arec 153 figures

\section{PARIS}

PAL L LCHEVALIER, 12, RUE de. тоскао $\left(\mathrm{vi}^{\circ}\right)$

1921

(Onvrage hoinoré d'une spbvention de l'Academie des Sciences de Paris)

(fondations $R$. Bonaparte ot Loutreuil) 


a

‥

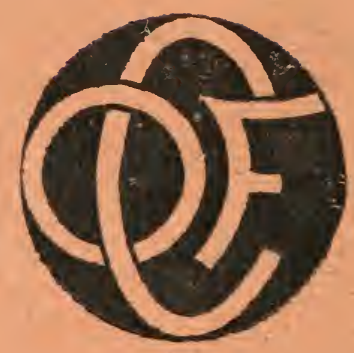




